Elektrische Quadrupolwechselwirkung in defektreichen und deformierten MAX-Phasen

Christoph Brüsewitz

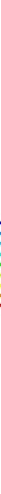





\title{
Elektrische Quadrupolwechselwirkung in defektreichen und deformierten MAX-Phasen
}

\author{
Dissertation \\ zur Erlangung des mathematisch-naturwissenschaftlichen Doktorgrades \\ „Doctor rerum naturalium“ \\ der Georg-August-Universität Göttingen \\ im Promotionsprogramm PROPHYS \\ der Georg-August University School of Science (GAUSS)
}

vorgelegt von

Christoph Brüsewitz

aus Groß-Umstadt

Göttingen, 2015 


\section{Betreuungsausschuss:}

Prof. Dr. Hans C. Hofsäss, II. Physikalisches Institut, Georg-August-Universität Göttingen

Prof. Cynthia A. Volkert Ph.D., Institut für Materialphysik,

Georg-August-Universität Göttingen

Dr. Ulrich Vetter, II. Physikalisches Institut,

Georg-August-Universität Göttingen

\section{Mitglieder der Prüfungskommission:}

Referent: $\quad$ Prof. Dr. Hans C. Hofsäss, II. Physikalisches Institut, Georg-August-Universität Göttingen

Korreferentin: Prof. Cynthia A. Volkert Ph.D., Institut für Materialphysik, Georg-August-Universität Göttingen

2. Korreferent: Prof. Dr. Peter Blaha, Institut für Materialchemie, Technische Universität Wien

\section{Weitere Mitglieder der Prüfungskommission:}

PD Dr. Jörn Große-Knetter, II. Physikalisches Institut, Georg-August-Universität Göttingen

Prof. Dr. Wolfram Kollatschny, Institut für Astrophysik, Georg-August-Universität Göttingen

Prof. Dr. Hans-Ulrich Krebs, Institut für Materialphysik, Georg-August-Universität Göttingen

Prof. Dr. Astrid Pundt, Institut für Materialphysik, Georg-August-Universität Göttingen

\section{Tag der mündlichen Prüfung:}

22. Juli 2015 
Für meinen Vater. 



\section{Inhaltsverzeichnis}

1 Einleitung 1

2 Grundlagen und Stand der Forschung 5

2.1 Eigenschaften der MAX-Phasen $\ldots \ldots \ldots \ldots \ldots$

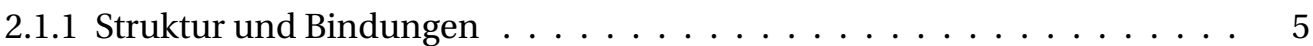

2.1.2 Mechanische Eigenschaften . . . . . . . . . . . . . . . 6

2.1 .3 Stand der Forschung . . . . . . . . . . . . . . . . . . . . . 11

2.2 Gradient-elastische Tensoren . . . . . . . . . . . . . . . . . . . . . 13

2.2.1 Eigenschaften der beteiligten Tensoren $\ldots \ldots \ldots \ldots \ldots$

2.2 .2 Stand der Forschung . . . . . . . . . . . . . . . . . . . 15

2.2 .3 Alternative Konzepte . . . . . . . . . . . . . . . . . . . . 17

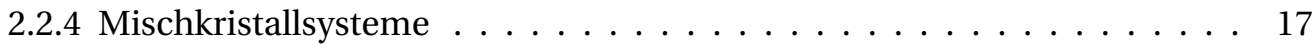

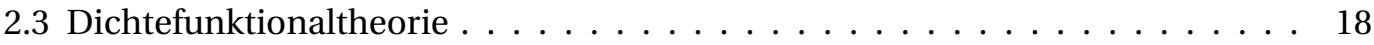

2.4 Ziele der Arbeit $\ldots \ldots \ldots \ldots$

3 Experimentelle Methoden 23

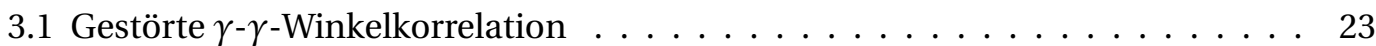

3.1.1 Grundzüge der PAC-Methode . . . . . . . . . . . . . . . . . . . . . . 24

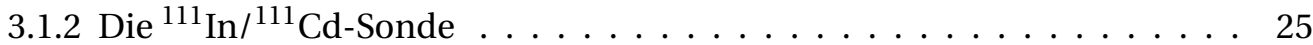

3.1 .3 Messaufbau . . . . . . . . . . . . . . . . . . . . . 28

3.1.4 Datenauswertung . . . . . . . . . . . . . . . . . 30

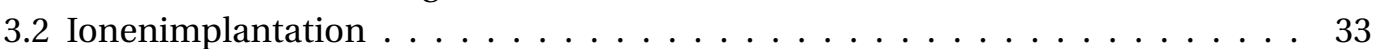

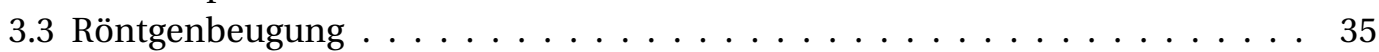

3.4 Transmissionselektronenmikroskop . . . . . . . . . . . . . . . 36

3.5 Synthese der MAX-Phasen . . . . . . . . . . . . . . . . . 37

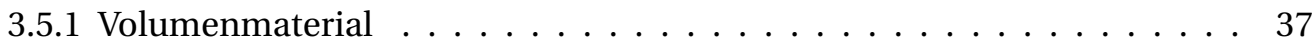

3.5 .2 Dünne Schichten . . . . . . . . . . . . . . . . . . . . 38

4 Theoretische Ergebnisse $\quad 39$

4.1 Bestimmung Gradient-elastischer Tensoren . . . . . . . . . . . . . . . . 39

4.1.1 Vereinfachungen durch die Punktgruppe der Sonde . . . . . . . . . . . 40

4.1.2 Ab-initio Berechnung der unabhängigen Konstanten . . . . . . . . . . . 41

4.2 Verteilungen des elektrischen Feldgradienten . . . . . . . . . . . . . . . 49

4.2 .1 Punktdefekte . . . . . . . . . . . . . . . . . . . . 50

4.2 .2 Versetzungen . . . . . . . . . . . . . . . . . . 52

4.2.3 Korngrenzen und andere höherdimensionale Defekte . . . . . . . . . . . 57

4.2.4 Elastische Verbreiterung . . . . . . . . . . . . . . . . . . . . . 57

4.2.5 Faltungsverhalten und experimenteller Zugang . . . . . . . . . . 62 
5 Experimentelle Ergebnisse $\quad 65$

5.1 Uniaxiale Kompression . . . . . . . . . . . . . . . . . . . . . . 67

5.1 .1 Qualitative Beschreibung . . . . . . . . . . . . . . . . . 67

5.1.2 Spannungsabhängigkeit der Quadrupolkopplungskonstante . . . . . . 70

5.1.3 Spannungsabhängigkeit der Verteilungsbreite . . . . . . . . . . . . 72

5.2 Dünnschichtsysteme . . . . . . . . . . . . . . . . . . 73

5.2.1 Elektrische Quadrupolwechselwirkung in Dünnschichtsystemen . . . . . 74

5.2.2 Temperaturabhängigkeit der Quadrupolkopplungskonstante . . . . . . . 75

5.3 Mischkristallsysteme . . . . . . . . . . . . . . . . . . 76

5.3.1 Synthese und Charakterisierung von $\mathrm{Ti}_{2}\left(\mathrm{Al}_{0,5}, \mathrm{In}_{0,5}\right) \mathrm{C} \ldots \ldots \ldots 77$

5.3.2 Elektrische Quadrupolwechselwirkung in Mischkristallsystemen . . . . . 78

5.3.3 Temperaturabhängigkeit der Quadrupolkopplungskonstante . . . . . . 80

5.3.4 Vergleich zu reinen MAX-Phasen . . . . . . . . . . . . . . . . . 82

6 Diskussion $\quad 87$

6.1 Ursache der Dehnungsabhängigkeit des Feldgradienten . . . . . . . . . . . . 87

6.2 Genauigkeit der ermittelten Gradient-elastischen Konstanten . . . . . . . . 90

6.2.1 Direkter Vergleich zu experimentellen Werten . . . . . . . . . . . . 90

6.2.2 Vergleich mit abgeleiteten Größen . . . . . . . . . . . . . . . . 91

6.2 .3 Relevante Fehlerquellen . . . . . . . . . . . . . . . . . . 93

6.3 Volumen- und Strukturabhängigkeit des Feldgradienten _ . . . . . . . . . . 95

6.4 Linear-elastische Störungen des Feldgradienten . . . . . . . . . . . . . . . 97

6.4.1 Auswirkungen der Deformation . . . . . . . . . . . . . . . . . . . . 98

6.4.2 Bestimmung von Versetzungsdichten . . . . . . . . . . . . . . . . 99

6.4.3 EFG-Verbreiterung unter Last . . . . . . . . . . . . . . . . . . 101

6.4 .4 Dünnschichtproben . . . . . . . . . . . . . . . . . . . . 103

6.5 Störungen fernab der linear-elastischen Theorie . . . . . . . . . . . . . . 103

6.6 Methoden zur Bestimmung interner Spannungen im Vergleich . . . . . . . . . 106

$\begin{array}{lll}7 & \text { Zusammenfassung und Ausblick } & 109\end{array}$

Anhang

$\begin{array}{ll}\text { A Details der DFT-Rechnungen } & 115\end{array}$

$\begin{array}{ll}\text { B Dehnungsfelder um Versetzungen } & 117\end{array}$

$\begin{array}{lr}\text { Literaturverzeichnis } & 119\end{array}$

$\begin{array}{ll}\text { Abbildungsverzeichnis } & 135\end{array}$

$\begin{array}{ll}\text { Tabellenverzeichnis } & 137\end{array}$

$\begin{array}{ll}\text { Publikationen } & 139\end{array}$

$\begin{array}{ll}\text { Lebenslauf } & 140\end{array}$

$\begin{array}{ll}\text { Danksagung } & 141\end{array}$

viii 


\section{Kapitel 1}

\section{Einleitung}

Die Suche nach neuen Struktur- und Funktionswerkstoffen wird oft durch die Anforderung an eine spezielle Materialeigenschaft, wie etwa eine besonders hohe Schmelztemperatur, einen minimalen Wärmeausdehnungskoeffizienten oder eine möglichst hohe Sprungtemperatur, motiviert. Im Allgemeinen lassen sich diese speziellen Eigenschaften - wie alle anderen Materialeigenschaften auch - auf den grundlegenden strukturellen und elektronischen Aufbau dieser Materialien zurückführen: Binäre Metallcarbide [1] weisen besonders starke kovalente Bindungen auf, die eine hohe thermische Stabilität zur Folge haben, die Eisen-Nickel-Legierung Invar [2] ändert ihr Volumen mit der Stärke der magnetischen Ordnung, die - temperaturabhängig - der normalen Wärmeausdehnung entgegenwirkt, und Hochtemperatursupraleiter [3] zeigen noch bei vergleichsweise hohen Temperaturen eine Korrelation der Leitungselektronen. Die tatsächliche Nutzbarkeit solcher Materialien hängt aber gleichzeitig immer auch von anderen Materialeigenschaften ab. Im Idealfall sind diese Eigenschaften einem anvisierten Verwendungszweck dienlich, wie etwa im Falle von Schneidwerkzeugen die Härte der Metallcarbide, häufig stehen sie aber auch einer unmittelbaren Anwendung entgegen, wie etwa die Sprödigkeit der Hochtemperatursupraleiter beim Bau eines supraleitenden Kabels.

Eine in dieser Hinsicht außergewöhnliche Kombination von Materialeigenschaften bieten die sogenannten MAX-Phasen, eine Klasse nanolaminarer, ternärer Komplexcarbide bzw. -nitride, die erstmals in den 1960er-Jahren von Jeitschko, Nowotny und Benesovsky [4-8] beschrieben wurden. Damals noch als H-Phasen bezeichnet, führte Barsoum 1999 den generalisierten Begriff der $\mathrm{M}_{n+1} \mathrm{AX}_{n}$-Phase ein [9], da er erkannte, dass sich über die $\mathrm{H}$-Phasen, wie zum Beispiel $\mathrm{Ti}_{2} \mathrm{AlC}$ [4], und dem strukturell verwandten $\mathrm{Ti}_{3} \mathrm{SiC}_{2}$ [10] hinweg mit dem zu diesem Zeitpunkt neu entdeckten System $\mathrm{Ti}_{4} \mathrm{AlN}_{3}$ eine Systematik ergibt, die durch die Anzahl $n$ eingebauter Metallcarbid- bzw. Metallnitridschichten innerhalb angrenzender A-Schichten charakterisiert wird. M steht hierbei für ein frühes Übergangsmetall, A vorwiegend für Elemente aus den Gruppen 13 bis 15 und X für Kohlenstoff oder Stickstoff. Elemente, die MAX-Phasen bilden, sind in Abbildung 1.1 dargestellt. Bisher wurden 57 MAX-Phasen entdeckt, die sich untereinander zum Teil beliebig mischen lassen. Es ist davon auszugehen, dass noch nicht alle Phasen entdeckt wurden, wie die erfolgreiche Synthese der jüngsten MAX-Phase $\mathrm{Mn}_{2} \mathrm{GaC}$ [11] im Jahre 2014 zeigt; gleiches gilt für Mischkristalle, auch hier werden fortwährend neue mischbare Systeme entdeckt [12].

MAX-Phasen lassen sich bei hohen Drücken und hohen Temperaturen aus elementaren Pulvern oder Verbindungen als dichtes, polykristallines Volumenmaterial herstellen (siehe Kapitel 3.5). Einkristalle wurden bislang bis zu einer Größe von wenigen hundert Mi- 


\begin{tabular}{|c|c|c|c|c|c|c|c|c|c|c|c|c|c|c|c|c|c|}
\hline 1 & & & & M & Jber & angs & hetal & & & & & & & & & & 18 \\
\hline $\mathrm{H}$ & 2 & & & A & Ilem & nte d & $\mathrm{r} \mathrm{Gr}$ & ıрp & $2-16$ & & & 13 & 14 & 15 & 16 & 17 & $\mathrm{He}$ \\
\hline $\mathrm{Li}$ & $\mathrm{Be}$ & & & $\mathrm{X}$ & Kohle & astof & odeı & Stic & toff & & & B & C & $\mathrm{N}$ & $\mathrm{O}$ & $\mathrm{F}$ & $\mathrm{Ne}$ \\
\hline $\mathrm{Na}$ & $\mathrm{Mg}$ & 3 & 4 & 5 & 6 & 7 & 8 & 9 & 10 & 11 & 12 & $\mathrm{Al}$ & $\mathrm{Si}$ & $\mathrm{P}$ & $S$ & $\mathrm{Cl}$ & $\mathrm{Ar}$ \\
\hline $\mathrm{K}$ & $\mathrm{Ca}$ & $\mathrm{Sc}$ & $\mathrm{Ti}$ & V & $\mathrm{Cr}$ & $\mathrm{Mn}$ & $\mathrm{Fe}$ & $\mathrm{Co}$ & $\mathrm{Ni}$ & $\mathrm{Cu}$ & $\mathrm{Zn}$ & $\mathrm{Ga}$ & $\mathrm{Ge}$ & As & $\mathrm{Se}$ & $\mathrm{Br}$ & $\mathrm{Kr}$ \\
\hline $\mathrm{Rb}$ & $\mathrm{Sr}$ & $\mathrm{Y}$ & $\mathrm{Zr}$ & $\mathrm{Nb}$ & Mo & Tc & $\mathrm{Ru}$ & $\mathrm{Rh}$ & $\mathrm{Pd}$ & $\mathrm{Ag}$ & $\mathrm{Cd}$ & In & Sn & $\mathrm{Sb}$ & $\mathrm{Te}$ & I & $\mathrm{Xe}$ \\
\hline Cs & $\mathrm{Ba}$ & $\mathrm{La}$ & Hf & Тa & W & $\operatorname{Re}$ & Os & Ir & $\mathrm{Pt}$ & $\mathrm{Au}$ & $\mathrm{Hg}$ & $\mathrm{Tl}$ & $\mathrm{Pb}$ & $\mathrm{Bi}$ & Po & At & $\mathrm{Rn}$ \\
\hline $\mathrm{Fr}$ & $\mathrm{Ra}$ & Ac & $\mathrm{Rf}$ & $\mathrm{Db}$ & $\mathrm{Sg}$ & $\mathrm{Bh}$ & Hs & $\mathrm{M}$ & Ds & $\mathrm{Rg}$ & $\mathrm{Cn}$ & & & & & & \\
\hline
\end{tabular}

Abbildung 1.1: Übersicht der Elemente, welche die 57 bisher bekannten MAX-Phasen und ihre Mischkristalle bilden (nach Referenz [13]). Die M-Elemente sind jeweils frühe Übergangsmetalle, die meisten A-Elemente stammen aus Gruppe 13 bis 15 und X steht für Kohlenstoff oder Stickstoff.

krometern synthetisiert [14]. Die Herstellung dünner Schichten gelingt mittels verschiedener Beschichtungsverfahren der chemischen und physikalischen Gasphasenabscheidung in Form polykristalliner und epitaktischer Schichten [15, 16].

Standen bei Jeitschko et al. noch Synthese und Strukturbestimmung der MAX-Phasen im Vordergrund, wurde die technologische Bedeutung dieser Systeme erst dreißig Jahre später durch systematische materialwissenschaftliche Untersuchungen erkannt [17]. MAX-Phasen sind - wie ihre konstituierenden Metallcarbide - relativ leicht, elektrisch und thermisch leitfähig, vergleichsweise steif und thermodynamisch teilweise bis über $2000 \mathrm{~K}$ stabil, lassen sich im Gegensatz dazu aber durch ihre geringere Härte erheblich leichter mit konventionellen Werkzeugen bearbeiten, überstehen schockartige Temperaturwechsel problemlos und sind erstaunlich zäh [13]. Die besonderen mechanischen Eigenschaften lassen sich dabei direkt auf die nanolaminare Struktur der MAX-Phasen und die individuellen Bindungsstärken zurückführen. Die Details der zugrundeliegenden Mechanismen werden zum Teil noch kontrovers diskutiert [18, 19].

Ausgehend von der hohen Leitfähigkeit ${ }^{a}$ und der Hitzebeständigkeit in Kombination mit einer passivierenden Oxidschicht, die sich auf manchen MAX-Phasen bildet [21], wurden bisher Heizelemente [22] konstruiert; weitere Anwendungen, die potentiell von der elektrischen Leitfähigkeit und den tribologischen Eigenschaften profitieren, umfassen Gleitlager [23] und Schleifkonstakte [24]. In der Nukleartechnik wird aufgrund der thermischen und mechanischen Stabilität ein Einsatz der MAX-Phasen als Strukturwerkstoff oder als Teil der Brennstoffhülle erwogen [25].

Des Weiteren wurden bereits erste Charakterisierungen an aus MAX-Phasen hergestellten Mikro-Elektromechanischen Systemen vorgenommen [26] und temperaturbeständige

\footnotetext{
${ }^{a}$ Tatsächlich werden auch einige MAX-Phasen bei tiefen Temperaturen supraleitend. $\mathrm{Nb}_{2} \mathrm{SC}$ beispielsweise ist ein Supraleiter 2. Art und weist dabei eine Sprungtemperatur von $5 \mathrm{~K}$ auf [20].
} 
elektrische Kontakte auf Siliziumcarbid hergestellt, die sich mit dem Substrat im chemischen Gleichgewicht befinden [27]. Durch selektives Ätzen der A-Schicht der MAX-Phase entsteht sogenanntes MXene, eine zweidimensionale Anordnung von MX-Oktaedern, die unter anderem als neuartiges Elektrodenmaterial in Batterien, in elektronischen und photonischen Anwendungen oder als Sensor zum Einsatz kommen könnte [28, 29].

Bisherige Untersuchungsmethoden, die zum Verständnis der ungewöhnlichen Eigenschaften der MAX-Phasen beigetragen haben, umfassen unter anderem Elektronenmikroskopie [21, 30-33] und Röntgendiffraktometrie [19, 34-36], makroskopische und mikroskopische Druckversuche [17-19, 37], Nanoindentierung [19, 32, 38, 39] und Rechnungen im Rahmen der Dichtefunktionaltheorie (DFT) [40-42]. Einen neuen Ansatz verspricht die Methode der gestörten $\gamma$ - $\gamma$-Winkelkorrelation (PAC) [43], bei der durch eine Messung des elektrischen Feldgradienten (EFG) Aussagen über die Asymmetrie einer lokalen, den Kern umgebenden Ladungsverteilung möglich sind. Angesichts der kurzreichweitigen, aber höchst sensitiven elektrischen Quadrupolwechselwirkung werden dabei vollständig neue Einblicke in die Natur der MAX-Phasen erwartet, die aufgrund des hohen Durchdringungsvermögens der verwendeten $\gamma$-Strahlung auch temperaturabhängig oder unter dem Einfluss einer äußeren Spannung gewonnen werden können. Durch eine systematische Untersuchung mehrerer MAX-Phasen konnte in Kombination mit DFT-Rechnungen bereits gezeigt werden, dass die PAC-Sonde ${ }^{111} \mathrm{In} /{ }^{111} \mathrm{Cd}$ auf besondere Weise geeignet ist, eine Vielzahl unterschiedlicher MAX-Phasen gleichermaßen im Hinblick auf den Feldgradienten am A-Platz zu charakterisieren [44-47].

An die bisherigen PAC-Untersuchungen anknüpfend, wird in der vorliegenden Arbeit erstmals erforscht, inwieweit sich Abweichungen vom idealen Feldgradienten, die beispielsweise durch Defekte im Material oder durch äußere Spannungen hervorgerufen werden, zur Untersuchung von MAX-Phasen eignen. Hierbei wird eine quantitative Beschreibung der beobachteten Effekte angestrebt, um nach Möglichkeit anhand der Veränderung des Feldgradienten direkt Aussagen über Versetzungsdichten und andere interne Spannungen treffen zu können. Bisherige Angaben der Versetzungsdichte in MAX-Phasen basierten auf elektronenmikroskopischen Messungen, bei denen nur vergleichsweise kleine Ausschnitte der Probe selektiv untersucht wurden. Eine Untersuchung mittels der PACMethode würde den untersuchten Bereich um sechs Größenordnungen ausweiten.

Die für eine solche Analyse notwendigen Grundlagen - insbesondere die Kenntnis des sogenannten Gradient-elastischen Tensors [48], der die Dehnungsabhängigkeit des Feldgradienten beschreibt, und die Abhängigkeit des Feldgradienten von der lokalen Umgebung - werden in dieser Arbeit maßgeblich weiterentwickelt, da bisherige Limitierungen durch Rechnungen im Rahmen der Dichtefunktionaltheorie überwunden und fundamentale Einsichten in die strukturellen Abhängigkeiten des Feldgradienten gewonnen werden. Die Erkenntnisse, die dabei erlangt werden, sind in anderen Methoden der nuklearen Festkörperphysik unmittelbar einsetzbar, womit die Tragweite dieser Arbeit weit über die gezeigten PAC-Untersuchungen an MAX-Phasen hinausreicht.

Die Arbeit gliedert sich in sieben Kapitel, wobei in Kapitel 2 die relevanten Grundlagen sowie der jeweilige Forschungsstand der Themenkomplexe MAX-Phasen, Gradientelastische Tensoren und Dichtefunktionaltheorie beschrieben werden. Daran anknüpfend folgt eine detaillierte Darstellung der Ziele dieser Arbeit. In Kapitel 3 werden die verwende- 
ten experimentellen Methoden beschrieben. Hierbei steht die PAC-Methode, mit der der elektrische Feldgradient gemessen wird, im Vordergrund; ergänzend kommen die Röntgendiffraktometrie und die Transmissionselektronenmikroskopie zur quantitativen Phasenanalyse und Mikrostrukturanalyse zum Einsatz.

In Kapitel 4 und 5 werden die theoretischen bzw. experimentellen Ergebnisse der Arbeit vorgestellt, Kapitel 6 befasst sich anschließend mit der Diskussion der zuvor erhaltenen Ergebnisse. Abschließend bietet Kapitel 7 eine Zusammenfassung der Resultate und skizziert zukünftige Verbesserungen und Anwendungen der vorgestellten Methoden und Ergebnisse. 


\section{Kapitel 2}

\section{Grundlagen und Stand der Forschung}

Eine zentrale Säule der hier durchgeführten Untersuchungen an MAX-Phasen bildet der Gradient-elastische Tensor, welcher die Dehnungsabhängigkeit des elektrischen Feldgradienten beschreibt, und in dieser Arbeit im Rahmen der Dichtefunktionaltheorie berechnet wird. Die relevanten Grundlagen der drei Themenkomplexe MAX-Phasen, Gradientelastischer Tensor und Dichtefunktionaltheorie werden nachfolgend dargestellt. Ausgehend von dem jeweiligen Forschungsstand werden am Ende dieses Kapitels die Ziele dieser Arbeit formuliert.

\subsection{Eigenschaften der MAX-Phasen}

Im Folgenden werden die für diese Arbeit relevanten Aspekte Struktur und Bindungen sowie mechanische Eigenschaften näher beschrieben. Eine umfassende Übersicht über diese und andere Eigenschaften der MAX-Phasen findet sich in einer Monographie von Barsoum, die im Jahre 2013 erschienen ist [13].

\subsubsection{Struktur und Bindungen}

Alle MAX-Phasen kristallisieren in einem hexagonalen Kristallsystem der Raumgruppe $P 6_{3} / m m c$ (Nr. 194). Innerhalb der MAX-Phasen bilden sich MX-Oktaeder aus, die durch A-Schichten getrennt werden. Die Anzahl der zwischen den A-Schichten liegenden MXOktaederschichten wird durch den Index $n$ bestimmt, wodurch das $c / a$-Verhältnis mit zunehmenden $n$ ansteigt. Die Einheitszellen der $\mathrm{M}_{n+1} \mathrm{AX}_{n}$-Phasen mit $n=1-3$ sind in Abbildung 2.1 dargestellt.

Für $n=2$ existieren zwei, für $n=3$ drei kristalline Modifikationen, die alle die Raumgruppe $P 6_{3} / \mathrm{mmc}$ aufweisen und sich einzig durch eine verschobene Stapelfolge unterscheiden $^{a}$. In der Regel wird in allen Systemen die $\alpha$-Modifikation angetroffen, allerdings sind für $n=2$ bzw. $n=3$ die $\beta$ - bzw. $\gamma$-Modifikation energetisch nur geringfügig ungünstiger und durch verschieben der A-Schicht leicht zugänglich [41, 42]. Die $\beta$-Modifikation für $n=3$ ist energetisch am ungünstigsten; eine Transformation hin zu dieser Modifikation gelingt nur durch Umstrukturieren der MX-Oktaeder [41].

Zwischen den M- und den X-Elementen bilden sich starke kovalente Bindungen aus, die durch die M-d- und X-p-Orbitale gebildet werden [42, 46]. Die Bindungen zwischen

${ }^{a}$ Alle hier untersuchten MAX-Phasen weisen einen Index $n=1$ auf, womit keine Polymorphie auftritt. 
(a)

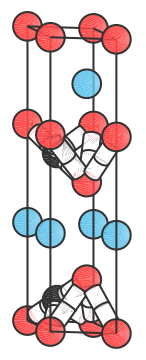

(b)

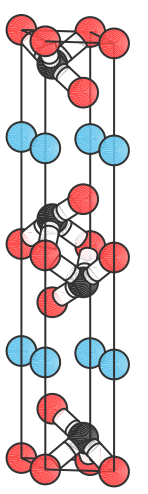

(c)

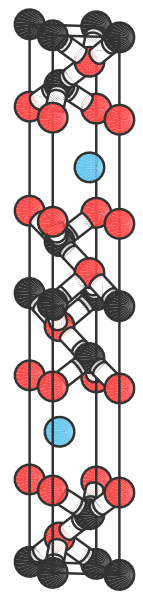

(d)

\begin{tabular}{ccccc}
\hline $\mathrm{M}_{2} \mathrm{AX}$ & Wyckoff- & \multicolumn{3}{c}{ Koordinaten } \\
& Position & $x$ & $y$ & $z$ \\
\hline $\mathrm{M}$ & $4 f$ & $1 / 3$ & $2 / 3$ & $z_{M}{ }^{a}$ \\
$\mathrm{~A}$ & $2 d$ & $1 / 3$ & $2 / 3$ & $3 / 4$ \\
$\mathrm{X}$ & $2 a$ & 0 & 0 & 0 \\
\hline \multicolumn{2}{c}{$a_{z_{M} \approx 0,082-0,086 .}$}
\end{tabular}

Abbildung 2.1: Einheitszellen der $\mathrm{M}_{n+1} \mathrm{AX}_{n}$-Phasen mit $n=1$ (a), $n=2$ (b) und $n=3$ (c) in $\alpha$ Modifikation. Rote Kugeln entsprechen den M-Elementen, blaue den A-Elementen und schwarze den X-Elementen. Die Anzahl der MX-Oktaeder zwischen den ASchichten wird durch $n$ bestimmt. Für die hier relevanten MAX-Phasen mit $n=1$ sind zusätzlich die Wyckoff-Positionen und die dazugehörigen internen Koordinaten der Elemente angegeben (d).

M- und A-Elementen sind deutlich schwächer, werden aber analog aus den M-d- und Ap-Orbitalen gebildet. X- und A-Element bilden keinerlei Bindung aus [42, 46]. Alle MAXPhasen sind aufgrund der endlichen elektronischen Zustandsdichte an der Fermienergie elektrisch und thermisch leitfähig, zum Teil sogar leitfähiger als ihre Pendants der Metallcarbide bzw. -nitride [13]. Die elektrische Leitfähigkeit wird hierbei maßgeblich durch die d-Zustände der M-Elemente, zu kleinerem Teil aber auch von den p-Zuständen der AElemente getragen. Die elektronische Zustandsdichte von $\mathrm{Ti}_{2} \mathrm{AlN}$ ist exemplarisch in $\mathrm{Ab}$ bildung 2.2 gezeigt.

Aufgrund der Ähnlichkeit der Bindungsstruktur aller MAX-Phasen ist es möglich, eine Vielzahl von Substitutionsmischkristallen innerhalb der MAX-Phasen zu bilden. Hierbei gelingt es, sowohl auf dem M- als auch auf dem A- und dem X-Platz Atome zu mischen. Die Mischung der Atome des M-Platzes ist zumeist uneingeschränkt möglich, auf dem APlatz sinkt mit zunehmendem Unterschied der Hauptgruppe die Löslichkeit [47, 49]. Kohlenstoff und Stickstoff sind, so denn die jeweiligen reinen Phasen existieren, vermutlich vollständig löslich [36], andernfalls nur begrenzt [50]. Die Vegard'sche Regel, also die lineare Abhängigkeit der Gitterkonstanten als Funktion des Anteils $x$ der Komponenten, trifft im Rahmen des Messfehlers auf alle bisher untersuchten MAX-Phasen zu [13, 34-36].

\subsubsection{Mechanische Eigenschaften}

Bei der Verformung von Materialien treten unter Last elastische Effekte auf, die sich vollständig reversibel zurückbilden, und andere, die das Material permanent verformen. Reversible Effekte lassen sich in der Regel mit der linearen Elastizitätstheorie [51, 52] beschreiben. Irreversible Effekte gehen mit plastischen oder spröden Verformungen einher, 
(a)

(b)

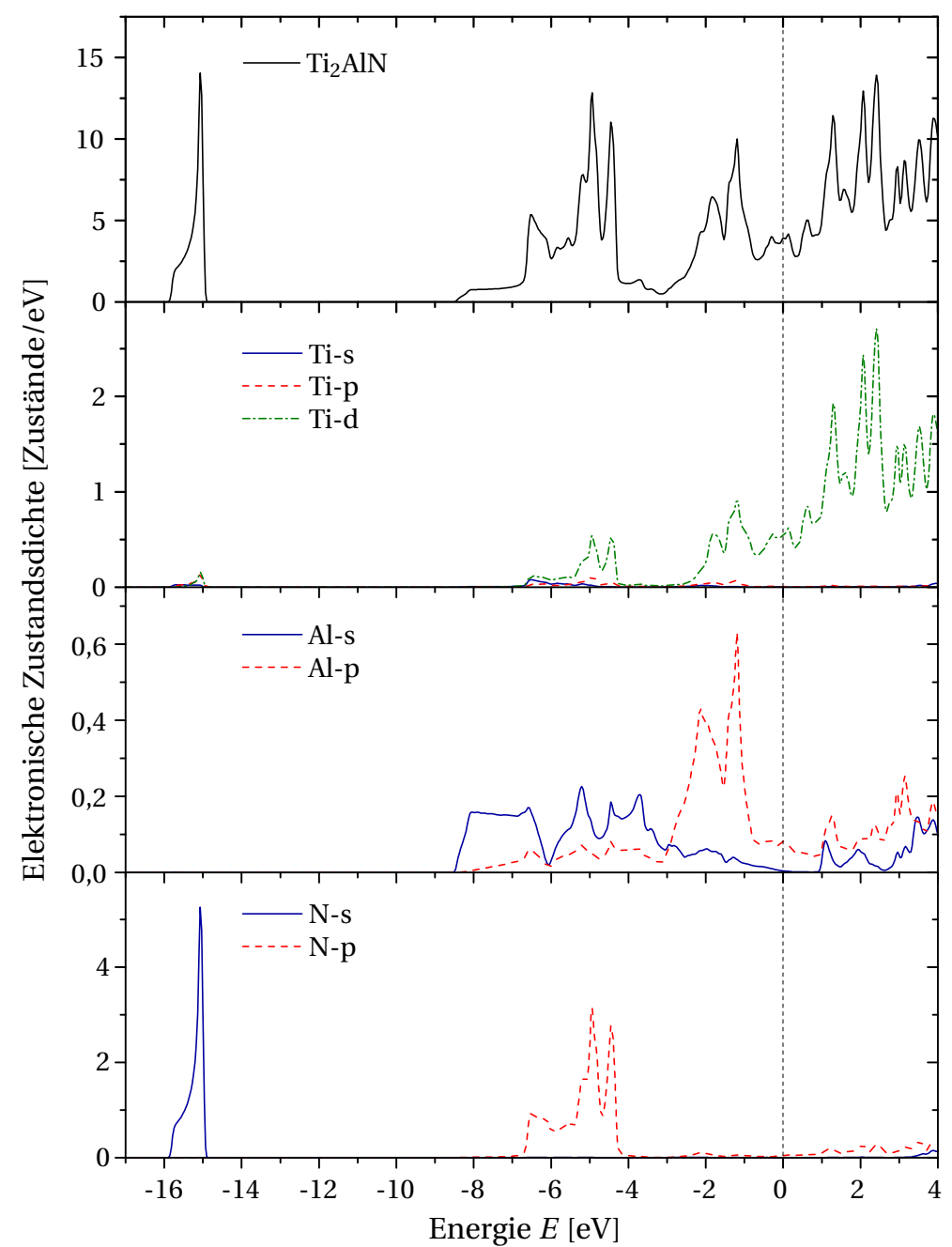

Abbildung 2.2: Elektronische Gesamtzustandsdichte (a) und lokale Partialzustandsdichten (b-d) in $\mathrm{Ti}_{2} \mathrm{AlN}$ relativ zur Fermienergie (berechnet mit WIEN2k, siehe Kapitel 4 und 6). Aus der endlichen Ti-d- und Al-p-Zustandsdichte an der Fermienergie resultiert die elektrische Leitfähigkeit der MAX-Phase.

die in Kristallen unmittelbar mit der Versetzungstheorie $[53,54]$ verknüpft sind. Im idealisierten Fall verhält sich ein Material bis zur Streckgrenze rein elastisch, darüber hinaus plastisch, bis die Bruchspannung erreicht wird und das Material versagt. Die Fähigkeit, sich über größere Dehnungen plastisch zu verformen, wird als Duktilität bezeichnet. Stoffe, die sich nur kaum plastisch Verformen lassen, sind spröde. Um eine plastische Verformung aufrecht zu erhalten, muss die augenblickliche Fließspannung überschritten werden; diese Spannung hängt im Allgemeinen über die Kaltverfestigung vom Grad der plastischen Verformung ab. Die Bruchspannung unter Dehnung, die Zugfestigkeit, ist im Allgemeinen von der Bruchspannung unter Kompression, der Druckfestigkeit, verschieden. Beginnend mit den elastischen Eigenschaften werden all diese Charakteristika der mechanischen Ei- 
Tabelle 2.1: Elastische Konstanten bei $T=0 \mathrm{~K}$ der MAX-Phasen und anderer untersuchten Materialien. Im Falle der MAX-Phasen liegen lediglich Vorhersagen aus Dichtefunktionalrechnungen vor, alle anderen Werte wurden experimentell bestimmt.

\begin{tabular}{ccccccc}
\hline & $c_{11}[\mathrm{GPa}]$ & $c_{12}[\mathrm{GPa}]$ & $c_{13}[\mathrm{GPa}]$ & $c_{33}[\mathrm{GPa}]$ & $c_{44}[\mathrm{GPa}]$ & Ref. \\
\hline $\mathrm{Cr}_{2} \mathrm{AlC}$ & 365 & 84 & 102 & 369 & 140 & {$[40]$} \\
$\mathrm{Nb}_{2} \mathrm{AlC}$ & 310 & 90 & 118 & 289 & 139 & {$[40]$} \\
$\mathrm{Ti}_{2} \mathrm{AlC}$ & 302 & 62 & 61 & 269 & 109 & {$[40]$} \\
$\mathrm{Ti}_{2} \mathrm{AlN}$ & 309 & 67 & 90 & 282 & 125 & {$[40]$} \\
$\mathrm{Ti}_{2} \mathrm{InC}$ & 282 & 65 & 55 & 240 & 86 & {$[40]$} \\
$\mathrm{Cd}$ & 131 & 40 & 41 & 57 & 24 & {$[55]$} \\
$\mathrm{Ti}$ & 176 & 87 & 68 & 191 & 51 & {$[56]$} \\
$\mathrm{Zn}$ & 179 & 36 & 54 & 68 & 56 & {$[57]$} \\
$\mathrm{ZnO}$ & 210 & 121 & 105 & 211 & 43 & {$[58]$} \\
$\mathrm{Al}$ & 123 & 71 & - & - & 31 & {$[59]$} \\
$\mathrm{Au}$ & 202 & 170 & - & - & 45 & {$[60]$} \\
$\mathrm{Cu}$ & 176 & 125 & - & - & 82 & {$[61]$} \\
$\mathrm{Mo}$ & 450 & 173 & - & - & 125 & {$[62]$} \\
$\mathrm{Nb}$ & 246 & 134 & - & - & 29 & {$[63]$} \\
$\mathrm{Ta}$ & 266 & 158 & - & - & 87 & {$[62]$} \\
\hline
\end{tabular}

${ }^{a}$ Messwerte bei Raumtemperatur.

genschaften nachfolgend für die MAX-Phasen beschrieben.

Aufgrund fehlender makroskopischer MAX-Phasen-Einkristalle konnte der Elastizitätstensor bisher nicht direkt vermessen werden, einzig die Struktur dieses symmetrischen Tensors ist bereits durch die Raumgruppe des Kristalls festgelegt. Einen guten Hinweise auf die Werte der Tensorelemente geben Dichtefunktionalrechnungen. Die fünf unabhängigen elastischen Konstanten $c_{m n}$ (siehe Kapitel 2.2 und Abbildung 4.1b) sind in Tabelle 2.1 exemplarisch für die hier untersuchten MAX-Phasen dargestellt. Ein Vergleich der aus diesen Konstanten mit dem Voigt'schen Ansatz [51], das heißt unter der Annahme uniformer Dehnung, berechneten Elastizitätsmoduln, Schubmoduln und Kompressionsmoduln mit den am Volumenmaterial gemessenen Größen, suggeriert eine Genauigkeit der Konstanten von ungefähr 10\% [40]. Die Elastizitätsmoduln der MAX-Phasen liegen üblicherweise zwischen 200 und $350 \mathrm{GPa}$, die Schubmoduln ungefähr zwischen 80 und $140 \mathrm{GPa}$; die Querkontraktionszahl variiert zwischen 0,15 und 0,29 [13].

Die Versetzungsbewegung ist in MAX-Phasen, außer unter extremen Bedingungen wie unter einem Nanoindenter [38], auf die Basalebene beschränkt. Der Burgersvektor von Schrauben- und Stufenversetzungen beträgt dabei 1/3〈11 20$\rangle$ [30]. Andere Versetzungen sind aufgrund des hohen $c / a$-Verhältnisses der MAX-Phasen unwahrscheinlich. MAXPhasen verhalten sich somit plastisch anisotrop. Die auftretenden Versetzungen sind bei Raumtemperatur mobil und weisen zu ungefähr gleichen Teilen Schrauben- und Stufencharakter auf [13]. Versetzungen, die auf der gleichen Basalebene liegen, stauen sich häufig an Hindernissen wie Korngrenzen auf; Versetzungen auf unterschiedlichen Basalebenen ordnen sich hingegen in Form von Versetzungswänden übereinander an [32, 39, 64, 65]. Die 

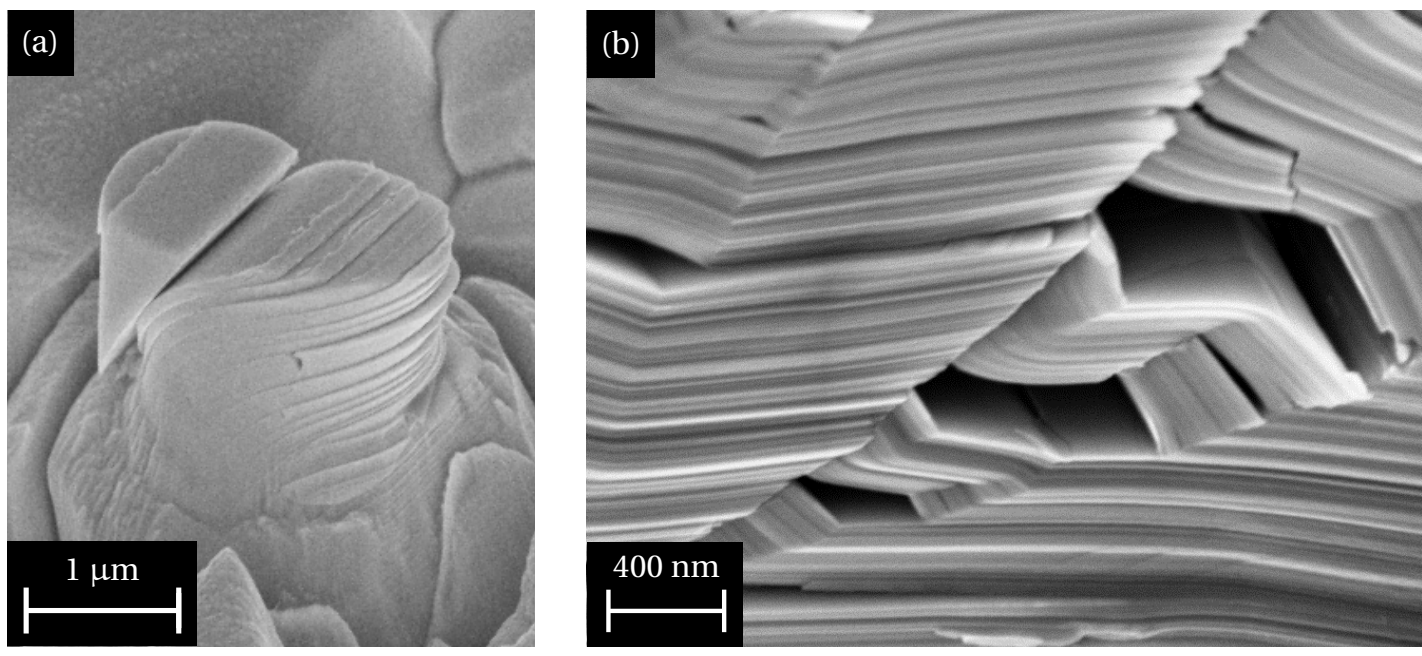

Abbildung 2.3: Typisches Verformungsverhalten von MAX-Phasen in (a) mikroskopischen, einkristallinen Säulen aus $\mathrm{Ti}_{4} \mathrm{AlN}_{3}$ [37] und (b) makroskopischem, polykristallinem $\mathrm{Ti}_{2} \mathrm{AlN}$ (abgebildet mit SEM LEO Supra 35). Versetzungsbasierte Verformung erfolgt nahezu ausschließlich auf den Basalebenen. Deren Abgleiten erfolgt ungehindert (obere Ecke der Säule), solange keine Hindernisse, wie etwa Korngrenzen, im Weg stehen. Sobald das Abgleiten behindert wird, entstehen Spannungen senkrecht zur Gleitebene, die zu Schichtverbiegungen, -aufspaltungen und -rissen führen.

Bestimmung von Versetzungsdichten erfolgte bisher ausschließlich über TEM-Messungen. Die auf diese Weise ermittelten Versetzungsdichten liegen dabei typischerweise im Bereich von $10^{13} \mathrm{~m}^{-2}$ in undeformierten und $10^{14} \mathrm{~m}^{-2}$ in deformierten Proben [30, 33, 64].

Die Beschränkung der Versetzungsbewegung auf die Basalebene limitiert die Anzahl unabhängiger Gleitsysteme auf zwei, womit eine homogene plastische Verformung von MAXPhasen-Volumenproben nicht möglich ist. Plastizität wird bei Raumtemperatur nur in geringem Maße beobachtet, bevor das Material versagt. Die Zugfestigkeit der MAX-Phasen liegt zwischen 100 und $300 \mathrm{MPa}$ und ist deutlich kleiner als die Druckfestigkeit, die zwischen 300 und $2000 \mathrm{MPa}$ liegt. Im gleichen Materialsystem zeigen feinkörnigere Proben höhere Festigkeiten als grobkörnigere [13].

Die auftretenden Verformungsmechanismen umfassen neben versetzungsbasiertem Abgleiten der Basalebenen auch deren Delaminationen sowie Schichtverbiegungen, Risse, Kornextrusion, Scherbänder und Kinkbänder [13]. Verformungszwillinge wurden dagegen bisher nicht beobachtet. Das Abgleiten der Basalebenen tritt schon bei im Vergleich zur Streckgrenze relativ kleinen Spannungen auf und wird erst durch Barrieren wie Korngrenzen verhindert [37]. Ist ein Abgleiten nicht möglich, bauen sich in der Schicht Normalspannungen auf, die zur Delamination, Schichtverbiegung und zu Rissen führen (siehe Abbildung 2.3a). Die Delamination entlang der Basalebenen wird durch die vergleichsweise schwachen M-A-Bindungen in der laminaren Struktur begünstigt. Kinkbänder stellen abgeknickte und teilweise delaminierte Bereiche der Probe dar, die häufig größere Risse stabilisierend überbrücken und dadurch maßgeblich zur mechanischen Stabilität der MAX-Phasen beitragen (siehe Abbildunge 2.3b). Der eigentlich Knick eines Kinkbands [66] 
besteht hierbei aus einer Versetzungswand, bildet also effektiv eine Kleinwinkelkorngrenze. Die Entstehung dieser charakteristischen Objekte ist, wie nachfolgend gezeigt wird, noch nicht vollständig verstanden [18, 19]. Die Kombination aller Mechanismen ermöglicht es den MAX-Phasen, sich vor dem Versagen begrenzt plastisch zu verhalten.

Ausgangspunkt einer kontrovers geführten Debatte bildet das Auftreten von Hysteresen im Spannungs-Dehnungs-Diagramm der MAX-Phasen [18, 19]. So zeigen sich unter zyklischer Kompression unterhalb der Streckgrenze Abweichungen vom linear-elastischen Verhalten, die sich in geschlossenen Schleifen zum Ausdruck bringen. Die Fläche innerhalb dieser Schleifen entspricht der Arbeit, die bei der Deformation dissipiert. Die Tatsache, dass bei einer Last unterhalb der Streckgrenze überhaupt Arbeit dissipiert, bedeutet zunächst einmal lediglich, dass schon vor Erreichen der Streckgrenze in Körnern mit hohem Schmid-Faktor ${ }^{a}$ Versetzungsbewegung stattfindet, was als solches im Einklang mit anderen Experimenten steht [31, 37]. Das Überraschende ist hierbei, dass sich die Deformation dabei nahezu vollständig reversibel zurückbildet, sich die bewegten Versetzungen also wieder in ihre Ausgangsposition begeben. Die MAX-Phase absorbiert also bei nicht zu hohen Spannungen Energie der Verformung, ohne sich dabei in signifikantem Maße irreversible zu verformen.

Eine erste, sehr einfache Erklärung dieses Verhaltens könnte im Aufstau schon existierender Versetzungen an Korngrenzen unter Last liegen, die beim Entlasten wieder in ihre Ausgangsposition zurück relaxieren. Der Beitrag eines solchen Aufstaus wird gemeinhin aber als zu klein eingeschätzt, da sich sonst signifikante reversible Hysteresen in deutlich mehr Materialien zeigen müssten [18, 19]. Neben diesem unzureichenden Ansatz existieren zwei weitere, zueinander konträre Erklärungen.

Barsoum et al. [18] führte 2003 zur Erklärung der reversiblen Hysteresen das Konzept der unvollständigen Kinkbänder (engl. incipient kink band, IKB) ein, die im Material in Form von Versetzungsringen entstehen sollen, welche mit zunehmender Last anwachsen, bis sie eine Korngrenze erreichen und schließlich eine mobile Versetzungswand, das heißt ein reguläres Kinkband, bilden. So lange der Versetzungsring noch nicht in Versetzungswände dissoziiert ist, soll er nach Wegnahme der Last wieder vollständig annihilieren. Der IKB-Mechanismus würde also zu einer unter Last vollständig reversiblen Nukleation von Versetzungen führen, die sich bewegen und dabei Arbeit verrichten.

Ein direkter Nachweis des IKB-Mechanismus blieb bisher aus. So wurden lediglich indirekte Hinweise, wie die ungefähr quadratische Abhängigkeit der dissipierten Arbeit von der maximalen Spannung sowie der Zunahme der dissipierten Arbeit mit zunehmender Korngröße und Porosität, herangezogen, die im Einklang mit der Theorie der Kinkbänder [66] stehen sollen [18, 68]. Einzig Methoden, die in der Lage sind, in-situ Versetzungen sichtbar zu machen, könnten überhaupt einen entsprechenden direkten Nachweis liefern. In-situ-TEM-Messungen würden hierbei allerdings aufgrund des begrenzten Probenvolumens ausscheiden, da entsprechende Versetzungsringe sofort an die Lamellenwand stoßen und nach der Belastung nicht mehr annihilieren würden [13]. Als Alternative kämen

\footnotetext{
${ }^{a}$ Körner mit hohem Schmid-Faktor sind so zur uniaxialen Last orientiert, dass die Versetzungsbewegung auf der Basalebene bei besonders kleinen Spannungen von statten geht. Der Schmid-Faktor selbst lässt es zu, bei gegebener äußerer Spannung die auf ein bestimmtes Gleitsystem wirkende Schubspannung zu ermitteln [67].
} 
Diffraktometriemethoden in Frage [13] - oder eben die hier verwendete PAC-Methode, bei der über den Feldgradienten indirekt auch Versetzungen quantitativ nachweisbar sein sollten.

Jones et al. [19] führte 2014 die reversiblen Hysteresen auf einen von den Kinkbändern unabhängigen Prozess zurück, der im Einklang mit klassischer Versetzungstheorie stehen und folglich auch ohne den exotischen IKB-Mechanismus auskommen soll. Der Grundgedanke ist der, dass sich im polykristallinen Material unter Last elastische Spannungen gleichermaßen in allen Körnern aufbauen, wobei die Körner mit hohem Schmitd-Faktor, das heißt mit den für das Abgleiten günstig orientierten Basalebenen, aber schon sehr früh Versetzungsbewegung zeigen, die zur beobachteten Dissipation der Energie führen. Dieser Aspekt ist insofern noch nicht besonders, als die Verformung eines jeden Polykristalls in den am günstigsten orientierten Körnern beginnt.

Die entscheidende Besonderheit der MAX-Phasen liege nun in der limitierten Anzahl an Gleitebenen, die dazu führen soll, dass sich Körner mit niedrigem Schmid-Faktor aufgrund ihrer Orientierung ausschließlich elastisch verformen und - sobald die Last weggenommen wird und sich diese Körner wieder entspannen - in den Körnern hohen Schmid-Faktors eine erneute, rückwärtsgerichtete Versetzungsbewegung induzieren, die es dem Polykristall ermöglichen soll nahezu vollständig in seine Ausgangslage zurück zu relaxieren. Auch bei dieser rückwärtsgerichteten Versetzungsbewegung würde wieder Energie dissipieren. Voraussetzung für dieses Verhalten ist neben der plastischen Anisotropie auch die geringe kritische Schubspannung des aktiven Gleitsystems, sodass schon geringe Spannungen ausreichen, Versetzungsbewegung zu induzieren.

Simulationen im Rahmen des EPSC-Modells (engl. elastic-plastic self-consistent model), bei dem die Deformation eines individuell orientierten Korns mit anisotropen elastischen Eigenschaften in einer homogenen Umgebung berücksichtigt wird, seien in der Lage unter der Annahme der plastischen Anisotropie bei geringer kritischer Schubspannung die beobachteten reversiblen Hysteresen und insbesondere die dabei dissipierte Arbeit, quantitativ korrekt zu reproduzieren [19]. Des Weiteren seien in-situ durchgeführte Röntgendiffraktometriemessungen im Einklang mit dem vorgeschlagenen Mechanismus [19], allein eine In-situ-Bestimmung der Versetzungsdichte blieb dabei aus. Die Entstehung der Kinkbänder sei statt auf den IKB-Mechanismus eher auf eine energetisch günstigere Anordnung regulärer Versetzungen benachbarter Basalebenen in Versetzungswänden zurückzuführen.

Insgesamt ergibt sich mit dem Ansatz von Jones et al. [19] also ein Bild, in dem die Existenz des IKB-Mechanismus zwar nicht eindeutig widerlegt wird, jedoch aber für die versetzungsbasierte, reversible Deformation und die Entstehung der Kinkbänder einfachere Erklärungen angeboten werden, die im Einklang mit der klassischen Versetzungstheorie stehen.

\subsubsection{Stand der Forschung}

Teile der experimentellen und theoretischen Grundlagen, die es in dieser Arbeit ermöglichen Unordnung und Deformation in MAX-Phasen mit Hilfe des elektrischen Feldgradienten zu untersuchen, wurden von Jürgens et al. [44-47] ab dem Jahre 2007 durch systematische Messungen mit der gestörten $\gamma$ - $\gamma$-Winkelkorrelation (siehe Kapitel 3.1) und Rechnun- 
Kapitel 2 Grundlagen und Stand der Forschung

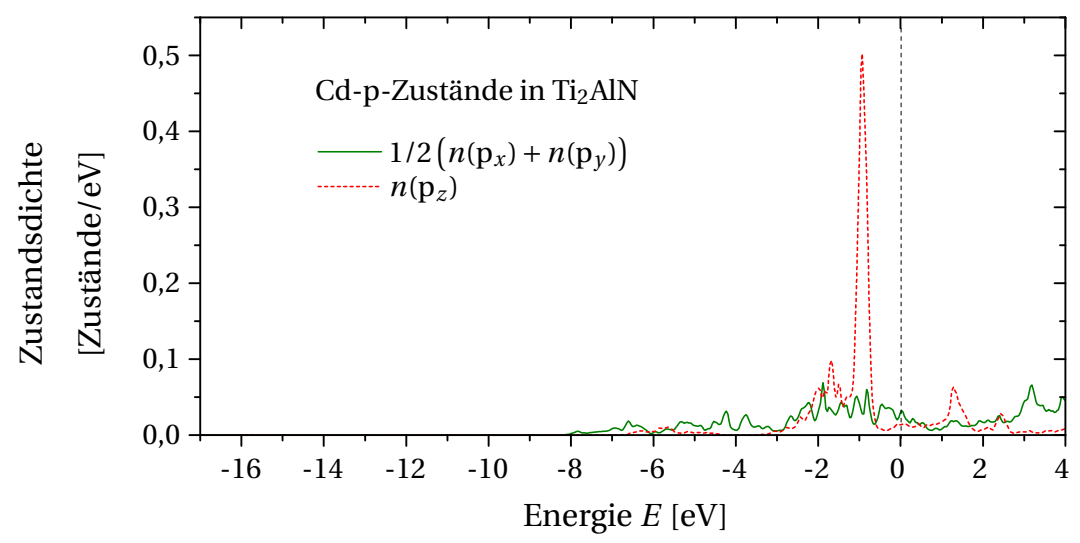

Abbildung 2.4: Lokale Partialzustandsdichte der p-Unterzustände des Cadmiums in $\mathrm{Ti}_{2} \mathrm{AlN}_{\text {relativ }}$ zur Fermienergie (berechnet mit WIEN2k, siehe Kapitel 4 und 6). Die Asymmetrie zwischen der Bestzungsdichte $n\left(\mathrm{p}_{z}\right)$ und $1 / 2\left(n\left(\mathrm{p}_{x}\right)+n\left(\mathrm{p}_{y}\right)\right)$ ist maßgeblich für den Feldgradienten an der Cadmiumsonde verantwortlich. Die Gesamtzustandsdichte aus Abbildung 2.2a ändert sich durch die Dotierung mit Cadmium nur geringfügig, größter Unterschied ist die starke Lokalisierung des $\mathrm{p}_{z}$-Zustandes der Cadmiumsonde im Vergleich zum substituierten Aluminium (siehe Abbildung 2.2c und Referenz [46]).

gen im Rahmen der Dichtefunktionaltheorie (siehe Kapitel 2.3) erarbeitet.

Es konnte gezeigt werden, dass sich die PAC-Sonde ${ }^{111} \mathrm{In} /{ }^{111} \mathrm{Cd}$ (siehe Kapitel 3.1) durch Ionenimplantation und anschließendem fünfstündigen Ausheilen der Proben bei $1173 \mathrm{~K}$ in den drei getesteten indium- und acht getesteten aluminiumhaltigen $\mathrm{M}_{n+1} \mathrm{AX}_{n}$-Phasen - unabhängig vom Index $n$ - auf dem A-Platz einbauen lässt [44-47]. Dies gelingt eingeschränkt überdies in manchen MAX-Phasen, die aus A-Elementen jenseits der Gruppe 13 aufgebaut sind, wie beispielsweise Germanium, Arsen oder Silizium. Der Anteil der Sonden, die auf dem A-Platz eingebaut werden, variiert zwischen $20 \%$ und $100 \%$. Je näher das A-Element mit Indium verwandt ist, desto effizienter ist der Einbau. Die gemessenen Feldgradienten sind gemäß der Symmetrie des A-Platzes axialsymmetrisch und weisen je nach MAX-Phase eine Verteilungsbreite von ungefähr $1 \%$ auf.

Des Weiteren konnte gezeigt werden, dass sich die PAC-Sonde ${ }^{181} \mathrm{Hf} /{ }^{181} \mathrm{Ta}$ durch Neutroneneinfang über die Reaktion ${ }^{180} \mathrm{Hf}(n, \gamma){ }^{181} \mathrm{Hf}$ direkt auf dem M-Platz der MAX-Phase $\mathrm{Hf}_{2}$ InC erzeugen lässt. Die Ionenimplantation von ${ }^{181} \mathrm{Hf}$ führte in keinem der untersuchten Systeme zu einer erfolgreichen Population des M-Platzes der MAX-Phasen [47].

Die Erkenntnis, dass die Indium-/Cadmiumsonde in den untersuchten Systemen auf dem A-Platz eingebaut wird, stammt einerseits aus der Beobachtung, dass alle gemessenen Feldgradienten ungefähr die gleiche Stärke aufweisen wie in $\mathrm{Ti}_{2} \mathrm{InC}$, in dem der Einbauort durch das native Indium vorgegeben ist, andererseits aus einem Vergleich der experimentell ermittelten Feldgradienten mit im Rahmen der Dichtefunktionaltheorie berechneten Feldgradienten [44-47]. Anhand der Dichtefunktionalrechnungen konnte auch gezeigt werden, dass der Feldgradient an der Cadmiumsone auf dem A-Platz vorwiegend durch eine asymmetrische Besetzung der p-Unterzustände des Cadmiums hervorgerufen 
wird. Die asymmetrische Besetzung der d-Zustände trägt hingegen in geringerem Maße zum Feldgradienten bei. Exemplarisch für die Cadmiumsonde in $\mathrm{Ti}_{2} \mathrm{AlN}$ ist in Abbildung 2.4 die asymmetrische Besetzung des p-Zustandes dargestellt. Im $\mathrm{p}_{z}$-Zustand - dem Zustand, der die M-A-Bindung bildet - befindet sich eine größere Elektronendichte als in der dazu senkrechten Basalebene, die durch die identisch besetzten $\mathrm{p}_{x}$ - und $\mathrm{p}_{y}$-Zustände gebildet wird [46]. Der Feldgradient ist folglich axialsymmetrisch und in Richtung $\langle 0001\rangle$ orientiert.

Die Stärke der Feldgradienten wurden außerdem temperaturabhängig vermessen, um zum einen durch eine Extrapolation hin zu $T=0 \mathrm{~K}$ Rechnung und Experiment vergleichen zu können, zum anderen aber auch, um den Temperaturverlauf mit anderen Materialien vergleichen zu können. Das experimentell ermittelte Maß für die Feldgradientenstärke, die Quadrupolkopplungskonstante $v_{Q}$ (siehe Kapitel 3.1), zeigte hierbei einen Verlauf, der mit dem empirischen Ausdruck

$$
v_{Q}(T)=v_{Q}(0 \mathrm{~K}) \cdot\left(1-\frac{B_{T}^{z z} \cdot T^{2}}{T+C_{T}^{z z}}\right)
$$

über alle MAX-Phasen hinweg beschrieben werden konnte [46]. Weder gelang es den Temperaturverlauf mit der in nicht-kubischen Metallen häufig beobachtete empirischen $T^{3 / 2}$ Abhängigkeit [69] anzupassen, noch mit einer linearen Abhängigkeit, wie sie beispielsweise in Metallen der seltenen Erden oder anderen intermetallischen Verbindungen beobachtet werden [70, 71]. Entweder wird dabei der lineare Verlauf bei hohen Temperaturen oder der abknickende Verlauf bei tiefen Temperaturen ungenügend reproduziert.

Ein wesentlicher Beitrag zur Temperaturabhängigkeit des Feldgradienten wird - wie in allen Materialien - durch Phononen verursacht, die den lokalen Feldgradienten durch die im Terahertzbereich liegende Oszillation der Atome zeitlich verändern; typische Quadrupolkopplungskonstanten liegen dagegen im Bereich von Megahertz, womit der gemessene Feldgradient bei $T>0 \mathrm{~K}$ ein zeitliches Mittel aller Konfigurationen darstellt [72]. Ein theoretisch fundierter Temperaturverlauf, der auf alle Materialien anwendbar ist, wurde noch nicht gefunden.

\subsection{Gradient-elastische Tensoren}

Das Konzept des Gradient-elastischen Tensors wurde 1957 von Shulman [48] in Analogie zum Elastizitätstensor eingeführt, um das Signal magnetischer Kernresonanzmessungen an Alkalihalogeniden unter elastischer Spannung beschreiben zu können. Statt den Spannungstensor an den Dehnungstensor zu koppeln, wie es bei dem Elastizitätstensor der Fall ist, schlug Shulman einen Tensor vor, der den elektrischen Feldgradienten an den Dehnungstensor koppelt. Beide Tensoren sind unmittelbar mit der Symmetrie der Punktgruppe des Gitters bzw. des Gitterplatzes verknüpft. Eine Übersicht über die gängigsten Tensordarstellungen physikalischer Kristalleigenschaften findet sich bei Nye [52]. Die in dieser Arbeit relevanten Tensoren werden im Folgenden näher erläutert. 


\subsubsection{Eigenschaften der beteiligten Tensoren}

Der elektrische Feldgradient $V_{i j}$ ist ein symmetrischer, spurfreier Tensor zweiter Stufe, der aus den zweiten räumlichen Ableitungen des elektrostatischen Potentials $\Phi$ gemäß

$$
V_{i j}=\frac{\partial^{2} \Phi}{\partial x_{i} \partial x_{j}}
$$

gebildet wird. Symmetrie und Spurfreiheit folgen unmittelbar aus der für das elektrische Potential geltenden Laplace-Gleichung und implizieren, dass kubische Ladungsverteilungen um einen Atomkern keinen elektrischen Feldgradienten (EFG) erzeugen. Aufgrund der Spurfreiheit des Tensors lässt sich der EFG im Hauptachsensystem durch die größte Komponente $V_{z z}$ und den Asymmetrieparameter $\eta=\left(V_{x x}-V_{y y}\right) / V_{z z}$ vollständig beschreiben [43]. Der EFG wird lokal am Atomkern durch seine Wechselwirkung mit dem Quadrupolmoment des Kerns gemessen.

Der Spannungstensor $\sigma_{i j}$ und der Dehnungstensor $\varepsilon_{i j}$ sind ebenfalls symmetrische Tensoren zweiter Stufe. In beiden Fällen folgt die Symmetrie aus der Konstruktion der jeweiligen Größe: Der Spannungstensor beschreibt Schub- und Scherspannungen eines Volumenelements, auf das kein Drehmoment wirkt; der Dehnungstensor ist über den Verschiebungsvektor $u_{i}$ durch

$$
\varepsilon_{i j}=\frac{1}{2}\left(\frac{\partial u_{i}}{\partial x_{j}}+\frac{\partial u_{j}}{\partial x_{i}}\right)
$$

symmetrisch definiert.

Die Kopplung zwischen den oben genannten Tensoren erfolgt für infinitesimale Änderungen über einen linearen Zusammenhang, der jeweils über einen Tensor vierter Stufe beschrieben werden kann. Da die Tensoren zweiter Stufe symmetrisch sind, reduziert sich die Anzahl der unabhängigen Komponenten der Kopplungstensoren von 81 auf 36. Dies erlaubt die Verwendung der Voigt'schen Notation [51, 52], in der die Tensoren vierter Stufe auf Matrizen zweiter Stufe reduziert werden und statt an Tensoren zweiter Stufe an Vektoren $\operatorname{koppeln}^{a}$. Die Elastizitätstensoren $c_{i j k l}$ und $s_{i j k l}$ verknüpfen in dieser linear-elastischen Theorie die Spannungs- und Dehnungstensoren in Voigt'scher Notation gemäß

$$
\sigma_{m}=\sum_{n=1}^{6} c_{m n} \varepsilon_{n} \text { und } \varepsilon_{m}=\sum_{n=1}^{6} s_{m n} \sigma_{n}
$$

Entsprechend wird der Zusammenhang zwischen der Änderung des EFGs und einer Spannung oder Dehnung über die Gradient-elastischen Tensoren $C_{i j k l}$ und $S_{i j k l}$ mit

$$
V_{m}-V_{m}^{0}=\sum_{n=1}^{6} S_{m n} \varepsilon_{n}=\sum_{n=1}^{6} C_{m n} \sigma_{n}, \begin{array}{ll}
1=x x, & 4=y z \\
2=z z, & 5=x z \\
3=x y
\end{array}
$$

${ }^{a}$ In der Voigt'schen Notation gilt $\varepsilon_{n}=\left(\varepsilon_{x x}, \varepsilon_{y y}, \varepsilon_{z z}, \gamma_{y z}, \gamma_{x z}, \gamma_{x y}\right)$ mit $\gamma_{i j}=2 \varepsilon_{i j}$. 
hergestellt. ${ }^{a}$ Die Indizes $m, n$ reichen von eins bis sechs, der EFG-Tensor $V_{i j}^{0}=V_{m}^{0}$ entspricht dem Tensor vor der Dehnung. Ohne Beschränkung der Allgemeinheit wird angenommen, dass sich dieser ursprüngliche EFG-Tensor im Hauptachsensystem befindet. Der aus einer Dehnung resultierende EFG-Tensor $V_{m}$ ist im Allgemeinen nicht im Hauptachsensystem sondern im Koordinatensystem von $V_{m}^{0}$ gegeben. Der Tensor $C_{m n}$ kann mit Hilfe von Gleichung 2.4 in $S_{m n}$ überführt werden und umgekehrt.

Neben der Symmetrie der beteiligten Tensoren zweiter Stufe lassen sich noch weiter Argumente finden, welche die unabhängigen Konstanten der Kopplungstensoren reduzieren. Im Falle der Elastizitätstensoren lässt sich aus der Energieerhaltung schließen, dass $s_{m n}$ und $c_{m n}$ symmetrisch sein müssen, womit die Anzahl der unabhängigen Komponenten auf 21 reduziert wird [51]. Dies gilt nicht für den Gradient-elastischen Tensor, allerdings lässt sich aus der Spurfreiheit des EFG-Tensors

$$
\sum_{m=1}^{3} S_{m n}=\sum_{m=1}^{3} C_{m n}=0
$$

folgern, was die Anzahl der unabhängigen Komponenten von 36 auf 30 reduziert [48].

Alle bisherigen Argumente gelten unabhängig von der Kristallklasse. Die Anwendung des Neumann'schen Prinzips [73], das besagt, dass Kristalleigenschaften die Symmetrieelemente der Punktgruppe des Kristalls beinhalten müssen, erlaubt es, die Kopplungstensoren noch weiter einzuschränken. Im Falle orthogonaler Kristallachsen existiert hierfür eine allgemeine Methode von Fumi [74], wohingegen in trigonalen und hexagonalen Systemen auf tabellierte Relationen der unabhängigen Elemente [75] zurückgegriffen werden kann. Die unabhängigen Elemente des Elastizitätstensor können so beispielsweise in kubischen Systemen auf zwei und in hexagonalen Systemen auf fünf elastische Konstanten reduziert werden. Das Neumann'sche Prinzip gilt auch für den Gradient-elastischen Tensor, allerdings kommt hier lediglich die Punktgruppe des betrachteten Kerns zum Tragen [48]. Shulman bestimmte so die Anzahl der unabhängigen Elemente des Gradient-elastischen Tensors in kubischen Systemen zu zwei. Für andere Systeme müssen die Symmetrieargumente aus Referenzen [74] oder [75] zunächst separat ausgewertet werden.

\subsubsection{Stand der Forschung}

Die experimentelle Bestimmung Gradient-elastischer Konstanten ist im Wesentlichen auf kubische Materialien beschränkt. Hierfür haben sich zwei Methoden etabliert, die beide auf Kernresonanzmessungen (engl. nuclear magnetic resonance, NMR) basieren. Der jeweils gemessene EFG wird dabei erst durch einen von außen induzierten Symmetriebruch erzeugt. Die erste Methode basiert auf den Experimenten von Shulman [48], in denen eine statische Änderung des EFGs durch eine uniaxiale Spannung entlang ausgewählter Kristallachsen hervorgerufen wird. Die zweite, genauere Methode greift auf die dynamische Wechselwirkung einer akustischen Welle mit dem EFG zurück (engl. nuclear acoustic resonance, NAR) [76]. Bisher untersuchte Materialklassen umfassen Alkalihalogenide [48, 76], Elementhalbleiter [77], III-V-Verbindungshalbleiter [78] und kubische Metalle [79-87].

In hexagonalen Systemen gibt es lediglich Messungen, die den Tensor unvollständig beschreiben. So wurde beispielsweise in Zinkoxid [88] und Cadmium [89] der EFG mit Hilfe 
der gestörten $\gamma$ - $\gamma$-Winkelkorrelation als Funktion einer uniaxialen Spannung entlang ausgewählter Kristallachsen vermessen. Diese Messungen stehen in unmittelbarem Zusammenhang zu einzelnen Komponenten von $C_{m n}$, erlauben es aber nicht, den gesamten Tensor zu bestimmen.

Diese deutliche Diskrepanz zwischen Untersuchungen in kubischen und anderen Systemen, sowohl in der Quantität der untersuchten Systeme als auch in der Vollständigkeit der bestimmten Konstanten, liegt im Wesentlichen in der zunehmenden Komplexität des Gradient-elastischen Tensors begründet. Genügen im kubischen Falle noch zwei Orientierungen der äußeren Spannung oder der akustischen Welle entlang kristallographisch ungleicher Achsen, sind in Fällen geringerer Symmetrie maximal Orientierungen entsprechend der Anzahl der unabhängigen Konstanten nötig, um den Tensor vollständig zu bestimmen. Dies ist eine unmittelbare Konsequenz aus der Tatsache, dass die Komponenten des EFGs experimentell nicht einzeln, sondern für gewöhnlich nur im jeweiligen Hauptachsensystem zugänglich sind. Unabhängig von der vorliegenden Symmetrie muss das Materialsystem immer als Einkristall vorliegen. Plastische Verformungen durch die Belastung müssen jeweils ausgeschlossen werden können.

Um die oben genannten Einschränkungen überwinden zu können, wäre es wünschenswert, Gradient-elastische Tensoren berechnen zu können. Für die Berechnung von elektrischen Feldgradienten stehen prinzipiell zwei Ansätze zur Verfügung, von denen der erste, das Punktladungsmodell, lediglich in ionischen Kristallen zu einigermaßen genauen Ergebnissen führt. Der zweite Ansatz basiert auf der vollständigen Berechnung der elektronischen Zustandsdichten des Festkörpers im Rahmen der Dichtefunktionaltheorie (siehe Kapitel 2.3) und ist dem ersten aufgrund der genaueren Vorhersage der berechneten Feldgradienten vorzuziehen. Der damit verbundene Rechenaufwand liegt zwar erheblich über dem des Punktladungsmodells, allerdings wird dieser Mehraufwand mit zunehmender Rechenleistung und gleichzeitiger Weiterentwicklung der zugrunde liegenden Theorien zusehends leichter zu bewältigen.

Konzeptionell wären beide Ansätze gleichermaßen geeignet, Gradient-elastische Konstanten zu bestimmen, da jeweils der vollständige EFG Tensor zugänglich ist und eine Dehnung durch eine Änderung der Atompositionen implementiert werden kann. Der Ansatz, Bandstrukturrechnungen zur Bestimmung der Gradient-elastischen Tensoren zu verwenden, wurde bisher lediglich im kubischen System Galliumphosphid an der Galliumsonde rudimentär verwendet [90]. Ein Vergleich zu experimentellen Daten oder eine Erweiterung zu komplexeren Systemen, beispielsweise in Form einer anderen Kristallklasse oder einem fremden Sondenatom, blieb dabei aus.

Aus dem Konzept des Gradient-elastischen Tensors ergeben sich Anwendungen insbesondere dort, wo Abweichungen vom regulären Gitter auftreten. Diese können beispielsweise durch elastische Verzerrungen um Punkt- oder Liniendefekte [91-93], in Grenzflächen [88] oder in Quantenpunkten [94-96] hervorgerufen werden. Hierbei sind besonders die Arbeiten von Kanert [91] in kubischen Systemen hervorzuheben, in denen es gelang, quantitative Aussagen über Versetzungsdichten zu treffen. Das Bindeglied zwischen Gradient-elastischem Tensor und Versetzungsdichte bildet hierbei eine Modellierung der EFG-Verteilung, wie sie im Umfeld von zufällig verteilten Versetzungen erwartet wird. Diese Form der Untersuchungen ist bisher auf kubische Systeme beschränkt geblieben. 


\subsubsection{Alternative Konzepte}

In Hinblick auf hexagonale und trigonale Metalle hat sich, unabhängig vom Gradientelastischen Tensor, ein Konzept etabliert, in dem die Änderung des elektrischen Feldgradienten mit der Änderung des Volumens und des $c / a$-Verhältnisses in Verbindung gebracht wird [89, 97]. Dieser Ansatz lässt sich auf verschiedene Variablen $\alpha_{i}$ anwenden, die auf spezifische Art Volumen und $c / a$-Verhältnis beeinflussen. Mögliche Variablen sind hierbei beispielsweise der Druck $p$, eine uniaxiale Spannung $\sigma_{u}$, die auf einen Einkristall wirkt, oder aber auch die Konzentration $x$ eines Mischkristalls. Für infinitesimale $\alpha_{i}$ zerfällt die relative Änderung der größten Komponente des Feldgradienten in eine Summe der Einzelbeiträge [89, 97]:

$$
\frac{1}{V_{z z}^{0}} \frac{\mathrm{d} V_{z z}}{\mathrm{~d} \alpha_{i}}:=\frac{\mathrm{d} \ln V_{z z}}{\mathrm{~d} \alpha_{i}}=\left(\frac{\partial \ln V_{z z}}{\partial \ln V}\right)_{c / a} \frac{\partial \ln V}{\partial \ln \alpha_{i}}+\left(\frac{\partial \ln V_{z z}}{\partial \ln c / a}\right)_{V} \frac{\partial \ln c / a}{\partial \alpha_{i}} .
$$

Basierend auf Messungen an Cadmium-Magnesium-Mischkristallen ${ }^{a}$ [98] sowie isostatisch und uniaxial belasteten Cadmium-Einkristallen [89, 99, 100] konnte ein linearer Zusammenhang zwischen EFG und $c / a$-Verhältnis festgestellt werden, der extrapoliert auf ein Verschwinden des EFGs bei ungefähr idealdichtester Packung $(c / a=1,633)$ hindeutet. Darauf aufbauend scheinen Änderungen des Volumens eine überproportionale Änderung des EFGs zur Folge zu haben [89].

Für kleine Dehnungen müssen dieser alternative Ansatz und der zuvor dargestellte Formalismus der Gradient-elastischen Konstanten a priori zu den gleichen Vorhersagen führen, da sich sowohl Änderungen des Volumens als auch des $c / a$-Verhältnisses durch einem Dehnungstensor ausdrücken lassen. Zur Beschreibung beliebiger Dehnungen wird hingegen der Gradient-elastische Tensor benötigt, da nur die Tensordarstellung Symmetriebrüche beschreiben kann, wie sie beispielsweise bei einer Dehnung entlang der Basalebenen oder bei einer Scherung vorkommen. Der Gradient-elastische Tensor bildet also den allgemeinsten Zusammenhang zwischen EFG und Dehnung ab.

\subsubsection{Mischkristallsysteme}

Der oben vorgestellte, alternativ Ansatz suggeriert, man könne mit einer linear-elastischen Theorie auch Feldgradienten in Mischkristallsystemen ${ }^{b}$ beschreiben. Dies ist nur bedingt der Fall. Zwar ändern sich in den meisten Mischkristallsystemen die Gitterkonstanten gemäß der Vegard'schen Regel ungefähr linear mit der Konzentration $x$, womit zur Änderung des EFGs auch linear-elastische Effekte gemäß Gleichungen 2.5 oder 2.7 beitragen, allerdings führt die zunehmende Konzentration an Fremdatomen auch zu einer von den elastische Effekten unabhängigen Änderung der elektronischen Struktur, die schlussendlich

\footnotetext{
${ }^{a}$ Durch sukzessive Erhöhung des Magnesiumanteils in $\mathrm{Cd}_{1-x} \mathrm{Mg}_{x}$-Mischkristallen wurde das $c / a$ Verhältnis verändert [98]. Der unbekannte Einfluss der Konzentration $\left(\partial \ln V_{z z} / \partial x\right)_{c, a}$ (siehe Kapitel 2.2.4) wurde bei der Analyse als vernachlässigbar eingeschätzt.

${ }^{b}$ Messungen an Mischkristallen dürfen nicht mit Messungen an intermetallischen Verbindungen verwechselt werden, da dort aufgrund der Überstruktur bzw. der veränderten Kristallklasse neue, für die intermetallische Verbindung charakteristische Feldgradienten auftreten [101, 102]. In diesem Sinne sind auch die MAXPhasen selbst intermetallische Verbindungen.
} 
auch den EFG ändert. Die Stärke dieser Änderung hängt maßgeblich von der lokalen Umgebung des Kerns ab, der den Feldgradienten detektiert. Da nun aber diese Umgebung in einem Mischkristall zufällig besetzt ist und der Feldgradient immer an einem Ensemble an Kernen vermessen wird, bildet sich folglich eine Verteilung des Feldgradienten aus.

Diese Verteilung wird zweifelsohne einen Mittelwert aufweisen, der sich mit der Konzentration $x$ ändert. Es liegt nahe, die Änderung linear zu nähern und beispielsweise an Gleichung 2.7 als zusätzlichen Beitrag $\left(\partial \ln V_{z z} / \partial x\right)_{c, a}$ anzufügen [97]. Dieser Schritt ist bedenklich, da er das fundamentale Konzept der elastischen Antwort des EFGs auf eine Weise mit der Feldgradientenverteilung vermischt, die der Komplexität des Problems nicht gerecht wird. Für infinitesimale Änderungen der Konzentration ist dieser Aspekt nicht weiter schlimm $^{a}$, für große Änderungen der Konzentration erscheint es dagegen sinnvoll, Mischkristalle vollständig losgelöst von der linear-elastischen Theorie des Felgradienten zu betrachten und die Feldgradientenverteilung mit der lokalen Umgebung direkt in Verbindung zu bringen.

Experimente an Mischkristallen zeigen bei kleinen Konzentrationen immer lineare Änderungen sowohl der Feldgradientenstärke als auch der zugehörigen Verteilungsbreite. Gemäß der obigen Erwartung ist davon auszugehen, dass sich diese Steigungen aus Beiträgen der linear-elastischen Theorie sowie der direkten Konzentrationsabhängigkeit zusammensetzen. In manchen Systemen setzt sich die Linearität der Stärke über den gesamten Konzentrationsbereich bis $x=1$ fort [103, 104], in anderen zeigt sich ein gekrümmter Verlauf [105]. Die Verteilungsbreite bildet bei maximaler Unordnung $x=0,5$ immer ein Maximum aus [103-106].

\subsection{Dichtefunktionaltheorie}

Die Beschreibung der elektronischen Struktur kondensierter Materie stellt ein quantenmechanisches Vielteilchenproblem dar, dessen Lösung unter anderem Vorhersagen über den elektrischen Feldgradienten an einem Kernort ermöglicht. In der Regel lässt sich dieses Problem nur näherungsweise lösen, eines der dabei benutzten Verfahren ist die Dichtefunktionaltheorie. Die im Folgenden dargestellten grundlegenden Konzepte dieses Themengebietes orientieren sich an der Übersicht von Cottenier [107].

Der exakte Hamiltonoperator des Festkörpers enthält Terme für die kinetische Energie aller Kerne und Elektronen sowie Terme für die Kern-Kern-, Kern-Elektron- und ElektronElektron-Wechselwirkung. Eine erste Vereinfachung dieses Hamiltonoperators bildet die Born-Oppenheimer-Näherung, bei der die Atomkerne aufgrund ihrer hohen Masse im Vergleich zu den Elektronen als unbeweglich angenommen werden. Das Problem reduziert sich dadurch auf die quantenmechanische Beschreibung der Elektronen und ihrer Wechselwirkung untereinander in einem durch die Atomkerne festgelegten Potential. Analog könnte für die Beschreibung der Elektron-Elektron-Wechselwirkung ein weiteres externes Potential eingeführt werden, um so das quantenmechanische Vielteilchenproblem auf mehrere Einteilchenprobleme zu reduzieren. Dieser Ansatz wird als Hartree-FockMethode bezeichnet und bildet die Grundlage der Molekülorbitaltheorie. Die Dichtefunk-

${ }^{a}$ In der betreffenden Referenz [97] wird der Anteil ohnehin als vernachlässigbar eingeschätzt. 
tionaltheorie geht hier einen anderen Weg, mündet aber auch in einer Darstellung, in der nur noch Einteilchenprobleme gelöst werden müssen.

Die Grundlage dafür bilden die Theoreme von Hohenberg und Kohn [108], die zeigen, dass es eine eindeutige Beziehung zwischen der Grundzustandsdichte der Elektronen und dem durch die Kerne vorgegebenen externen Potential gibt. Diese Elektronendichte umfasst alle Informationen, die auch in den eigentlichen Wellenfunktionen enthalten sind. Folglich sind alle Eigenschaften Funktionale dieser Dichte. Ziel der Dichtefunktionalrechnung ist es, diese Dichte für ein bestimmtes System zu bestimmen. Dies gelingt mit Hilfe der Kohn-Sham-Gleichungen [109] und einem von der Elektronendichte abhängigen Austausch-Korrelations-Potential, das die Wechselwirkung der Elektronen untereinander berücksichtigt. Die gekoppelten Differentialgleichungen vereinfachen sich so zu den ungekoppelten Kohn-Sham-Gleichungen, die nur noch von der Elektronendichte und vom unbekannten Austausch-Korrelationspotential abhängen. Da dieses Potential selbst eine Funktion der Dichte ist, wird eine selbstkonsistente Elektronendichte in einem iterativen Prozess gesucht. Für das Austausch-Korrelations-Potential können nur Näherungen angegeben werden. Die einfachste hängt hierbei nur von der lokalen Elektronendichte ab (engl. local density approximation, LDA), eine Erweiterung berücksichtigt außerdem auch den Gradienten der Dichte (engl. generalized gradient approximation, GGA) ${ }^{a}$

Zur Lösung der Kohn-Sham-Gleichung werden die darin enthaltenen Eigenfunktionen in Basisfunktionen zerlegt. Eine geschickte Wahl des Basissatzes ist hierbei entscheidend, um mit möglichst wenigen Basisfunktionen dem exakten Ergebnis nahe zu kommen. Ein möglicher Ansatz bieten hierbei ebene Wellen, die, als Bloch-Wellen formuliert, direkt die Periodizität des Gitters berücksichtigen. Die benutzten Basisfunktionen werden hierbei auf reziproke Gittervektoren beschränkt, die eine bestimmte Länge $K_{\max }$ nicht überschreiten. Dieses Eigenwertproblem muss für jeden k-Punkt, das heißt jeden reziproken Vektor, der ersten Brillouin-Zone separat gelöst werden. Die Anzahl der verwendeten k-Punkte bildet hierbei im Idealfall einen Kompromiss aus Genauigkeit und Rechenaufwand.

Nahe des Kerns wird die Beschreibung der Eigenfunktionen durch ebene Wellen ineffizient. Zwei konzeptionell verschiedene Ansätze können hierbei zur Effizienzsteigerung verwendet werden, wovon der erste Ansatz, die Einführung eines geglätteten Pseudopotentials in Kernnähe [112], insbesondere für Fragestellungen nach dem elektrischen Feldgradienten am Kernort ungeeignet ist. Der zweite, geeignetere Ansatz besteht darin, einen erweiterten Basissatz zu verwenden, bei dem die ebenen Wellen in Kernnähe durch Kugelflächenfunktionen ersetzt werden, die stetig an die ebenen Wellen des dazwischenliegenden Bereiches anschließen (engl. augmented plane wave, APW) [113]. Dieser kernnahe Bereich wird durch eine Kugel mit dem Radius $R_{\mathrm{MT}}$ (engl. muffin-tin sphere) um die Atomkerne beschrieben. Auch hier muss die Entwicklung der Wellenfunktion innerhalb der Kugel bei einer maximalen Quantenzahl $l_{\max }$ abgebrochen werden.

Als solche wird die APW-Methode nicht mehr verwendet, jedoch aber ihre beiden performanteren Erweiterungen $\mathrm{LAPW}^{b}[114]$ und APW+lo ${ }^{c}[115,116]$. Eine Kombination bei-

${ }^{a}$ In dieser Arbeit werden die Austausch-Korrelations-Potentiale von Perdew, Burke und Enzerhof (PBEGGA) [110] und von Wu und Cohen (WC-GGA) [111] verwendet.

${ }^{b}$ Engl. linearized augmented plane wave method, LAPW.

${ }^{c}$ Engl. augmented plane wave plus local orbitals method, APW+lo. 
der Erweiterungen kommt zur Beschreibung der Valenzelektronen im hier verwendeten Programmpaket WIEN2k $[117,118]$ zum Einsatz. Diese aufwändige Beschreibung ist für reine Kernzustände (engl. core state) nicht nötig. Folglich werden Kernzustände separat durch Wellenfunktionen freier Atome im Potential der Valenzelektronen beschrieben. Die Unterscheidung zwischen Valenz- und Kernzuständen wird durch die Separationsenergie $E_{\text {sep }}$ festgelegt. Insbesondere für Übergangsmetalle kann dieses Unterscheidungskriterium dazu führen, dass Elektronen gleicher Drehimpulsquantenzahl aber unterschiedlicher Hauptquantenzahl simultan als Valenzelektronen berücksichtigt werden. In diesen Fällen ist es sinnvoll, den energetisch niedrigeren, gebundenen Zustand als sogenanntes lokales Orbital (engl. local orbital, LO) gesondert zu behandeln [119]. Diese Art von Zuständen werden häufig als Semikernzustände (engl. semi-core state) bezeichnet.

Da in dieser Art von Rechnungen bis auf Atompositionen keine empirischen Informationen über das Material benötigt werden, lassen sich Elektronendichten ab-initio berechnen. Ausgehend von der Elektronendichte können dann beispielsweise Zustandsdichten, Bandstrukturen oder eben der elektrische Feldgradient ermittelt werden. Auf diese Weise können elektrische Feldgradienten beispielsweise in Metallen [120], Halbleitern [121] oder eben auch MAX-Phasen [46, 47] mit einer Genauigkeit von ungefähr 10\% reproduziert werden. Die Auswertung des EFGs erfolgt direkt aus einem mit $1 / r^{3}$ gewichteten Integral über die asymmetrische Elektronendichte um den Kern [122, 123]. Anhand des Wichtungsfaktors mit dem Abstand vom Kern wird deutlich, dass Elektronen in unmittelbarer Nähe des Kerns verstärkt zum EFG beitragen.

\subsection{Ziele der Arbeit}

Ziel der Arbeit ist es, den Einfluss von Unordnung und Deformationen auf den elektrischen Feldgradienten zunächst quantitativ zu verstehen und darauf aufbauend Aussagen über die in MAX-Phasen enthaltenen Defekte zu treffen.

Um dieses Ziel zu erreichen, wird erstmals eine Methode vorgestellt, wie in Kristallklassen beliebiger Symmetrie - auch ohne Zugang zu Einkristallen - der Gradient-elastische Tensor bestimmt werden kann. Hierzu wird in Kapitel 4.1 für verschiedene kubische und hexagonale Systeme exemplarisch die Struktur des Tensors durch eine Betrachtung der Punktgruppe ermittelt. Anschließend werden die unabhängigen Konstanten im Rahmen der Dichtefunktionaltheorie berechnet. Es werden 6 kubische Metalle untersucht, deren Gradient-elastische Konstanten bereits vermessen wurden [79, 81-87], die vier MAXPhasen $\mathrm{Nb}_{2} \mathrm{AlC}, \mathrm{Ti}_{2} \mathrm{AlC}$, $\mathrm{Ti}_{2} \mathrm{AlN}$ und $\mathrm{Ti}_{2} \mathrm{InC}$ sowie einige hexagonale Systeme, in denen von den Gradient-elastische Konstanten abgeleitete Größen zum Vergleich herangezogen werden können [88, 89, 99, 100, 124]. Anhand eines Vergleiches zwischen Rechnung und Experiment wird anschließend die zu erwartende Genauigkeit der mit dieser Methode ermittelten Konstanten abgeschätzt.

Des Weiteren werden durch die hier gezeigte theoretische Beschreibung entscheidende Einblicke erwartet, die zu einem detaillierten Verständnis der Dehnungsabhängigkeit des Feldgradienten beitragen. Hierzu existieren bisher nur rudimentäre Vorstellungen, die auf dem Versagen des Punktladungsmodells in der Beschreibung des Gradient-elastischen 
Tensors beruhen [91].

Sind die Gradient-elastischen Konstanten bekannt, lässt sich erstmalig die Vermutung, der Feldgradient hexagonaler Metalle würde bei ideal dichtester Packung verschwinden, direkt überprüfen. Gleichermaßen kann die vermutete überproportionale Volumenabhängigkeit des Feldgradienten untersucht werden.

Ausgehend von den Gradient-elastischen Konstanten wird in Kapitel 4.2 durch eine Monte-Carlo-Simulation erstmals die Verteilung des Feldgradienten in hexagonalen Systemen in der Umgebung verschiedener Defekte und unter dem Einfluss uniaxialer Spannungen, wie sie im experimentellen Teil auftauchen, untersucht. Vergleichbare Studien beschränken sich auf analytische Ansätze und kubische Systeme [91], womit ein direkter Transfer nicht möglich ist, da der bereits intrinsisch in hexagonalen Systemen vorhandene Feldgradient und die Anisotropie des Gradient-elastischen Tensors maßgeblichen Einfluss auf die resultierenden Verteilungen haben.

Die experimentelle Untersuchung dieser elastischen Effekte erfolgt durch ein Experiment, in dem erstmals mit der PAC-Methode die Deformation eines Materials in-situ beobachtet wird (siehe Kapitel 5.1). Unter Last überlagern sich hierbei die elastische Verbreiterung mit den internen Spannungen der durch die Verformung eingebrachten Defekte; nach Wegnahme der Last tragen nur noch Defekte zur Verteilung bei. Durch einen Vergleich mit der theoretischen Erwartung resultiert hieraus erstmals eine quantitative Analyse des Einflusses interner Spannungen auf den Feldgradienten in hexagonalen Systemen. Die untersuchten Materialien umfassen neben den vier schon oben genannten MAXPhasen auch Titan und Zink als Referenzsystem.

Ein zweites Experiment, in dem mit $\mathrm{Cr}_{2} \mathrm{AlC}$ erstmals ein Dünnschichtsystem der MAXPhasen mittels der PAC-Methode untersucht wird (siehe Kapitel 5.2), ist ebenfalls durch die Untersuchung elastischer Effekte motiviert: Die zentrale Frage ist hierbei, ob interne Spannungen in der Schicht, beispielsweise hervorgerufen durch die Grenzfläche zum Substrat oder durch thermisch induzierte Spannungen, die PAC-Messung beeinflussen.

Im dritten Experiment wird der direkte Einfluss von Defekten auf die elektronische Struktur untersucht, indem eine so große Defektdichte durch Fremdatome eingebracht wird, dass sich zwangsläufig die lokale Umgebung der Sonde ändert. Um dieses Ziel zu erreichen, werden aus den obigen vier MAX-Phasen Mischkristalle gebildet, wobei $\mathrm{Ti}_{2} \mathrm{AlC}$ das zentrale System bildet. Dies ermöglicht durch Beimischen jeweils einer weiteren MAXPhase eine systematische Substitution einzelner Gitterplätze. In diesem Zusammenhang wird erstmals die Synthese von $\operatorname{Ti}_{2}\left(\mathrm{Al}_{1-x}, \mathrm{In}_{x}\right) \mathrm{C}$ beschrieben. Vergleichbare Studien existieren in Hafnium-Zirkonium-Mischkristallen [105] und in Metalloxiden [103, 104]. Bemerkenswerterweise wird in beiden Fällen der Einfluss auf die elektronische Struktur praktisch nicht diskutiert, deren Relevanz sich in den hier durchgeführten Experimenten aber deutlich zeigt. 



\section{Kapitel 3}

\section{Experimentelle Methoden}

Die experimentelle Bestimmung des elektrischen Feldgradienten gelingt im Allgemeinen durch seine Wechselwirkung mit dem Quadrupolmoment eines Atomkerns. Zur Messung dieser elektrischen Quadrupolwechselwirkung stehen Werkzeuge der nuklearen Festkörperphysik [125] zur Verfügung, die im Wesentlichen in der Mitte des 20. Jahrhunderts entwickelt wurden. In dieser Hinsicht sind neben der hier verwendeten zeitlich differentiellen Beobachtung der gestörten $\gamma$ - $\gamma$-Winkelkorrelation (engl. time differential perturbed angular correlation, TDPAC oder PAC) [43, 126] die Kernresonanzspektroskopie [127-129], die Mößbauerspektroskopie [130, 131] sowie deren Variationen verbreitet. Welche dieser Methoden zum Einsatz kommt, hängt von der Fragestellung und den daraus resultierenden Anforderungen an das jeweilige Sondenatom ab. Die PAC-Methode eignet sich in besonderer Weise zur Untersuchung der MAX-Phasen, da die populäre Indium/Cadmium-Sonde in einer Vielzahl von verschiedenen MAX-Phasen auf einem definierten Gitterplatz, dem A-Platz, eingebaut werden kann [46, 47]. Hierdurch ergibt sich die Möglichkeit, mit Hilfe eines Messaufbaus verschiedene MAX-Phasen zu untersuchen und die Ergebnisse zu vergleichen. Die für diese Arbeit relevanten Grundlagen der PAC-Methode sowie die damit einhergehende Ionenimplantation werden im Folgenden erläutert.

Neben der gestörten $\gamma$ - $\gamma$-Winkelkorrelation wurden zur quantitativen Phasenanalyse und zur Mikrostrukturanalyse der untersuchten Materialien die Röntgendiffraktometrie [132-134] und die Transmissionselektronenmikroskopie [135, 136] eingesetzt. Beide Methoden werden im Anschluss an die PAC-Methode dargestellt. Des Weiteren wird die Herstellung der analysierten Proben geschildert. Die Synthese der in dieser Arbeit neu entdeckten MAX-Phase, $\mathrm{Ti}_{2}\left(\mathrm{Al}_{0,5}, \mathrm{In}_{0,5}\right) \mathrm{C}$, wird separat in Kapitel 5.3 beschrieben.

\subsection{Gestörte $\gamma-\gamma$-Winkelkorrelation}

Ausgehend von der Bestimmung von Kerneigenschaften mittels Winkelkorrelationsmessungen wurde 1940 von Hamilton [126] erkannt, dass bei bekannten Kerneigenschaften auch Aussagen über die am Kernort wirkenden elektromagnetischen Felder möglich sein müssen. Diese Erkenntnis kann gemeinhin als Geburtsstunde der PAC betrachtet werden. Über die letzten siebzig Jahre hinweg haben sich hieraus, häufig ergänzt durch Dichtefunktionalrechnungen, Anwendungen in der Strukturbestimmung von Molekülen [137], der Untersuchung von Phasenübergängen [138, 139] und Magnetismus [140], der Defektbestimmung in kubischen Materialien [141-144] oder der Bestimmung von Quadrupolmomenten von Kernzuständen [120] entwickelt. Ausführliche Beschreibungen der gestörten 
$\gamma-\gamma$-Winkelkorrelation finden sich beispielsweise bei Frauenfelder und Steffen [43] oder Schatz und Weidinger [125]. In diesem Unterkapitel folgen eine Erklärung der Methode, eine Beschreibung des Messaufbaus und -ablaufs sowie eine Übersicht über gängige Auswertungsverfahren.

\subsubsection{Grundzüge der PAC-Methode}

Die gestörte $\gamma$ - $\gamma$-Winkelkorrelation nutzt, wie bereits angedeutet, dass die Emission des zweiten Photons einer $\gamma$ - $\gamma$-Kaskade nicht unabhängig von der ersten, sondern unter einer Winkelverteilung $W(\theta)$ erfolgt. Diese Winkelverteilung wird durch die Kerneigenschaften bestimmt und lässt sich beispielsweise durch eine Koinzidenzmessung beider Photonen mit einem zueinander beweglichen Detektorpaar bestimmen. $W(\theta)$ lässt sich in eine Reihe von Legendre-Polynomen entwickeln und hängt gemäß

$$
W(\theta)=\sum_{k} A_{k k} P_{k}(\cos \theta)
$$

von den Anisotropiekoeffizienten $A_{k k}$ und vom Winkel $\theta$ ab [43]. Der Spin $I$ der beteiligten Kernzustände und die Drehimpulsquantenzahl $l$ der emittierten Strahlung bestimmen die auf $A_{00}=1$ normierten Anisotropiekoeffizienten [145] und limitieren den auf gerade Werte beschränkten Laufindex $k$ [146]. Wird der zeitliche Abstand $t$ zwischen den beiden Photonen der Kaskade unter einem fixen Winkel für ein Ensemble an Zerfällen vermessen, ergibt sich eine vom Startzeitpunkt $t=0$ an exponentiell abfallende Verteilung

$$
N(\theta, t) \propto W(\theta) \cdot \exp \left(-\frac{t}{\tau}\right),
$$

die auf die endliche Lebensdauer $\tau$ des Zwischenniveaus der Kaskade zurückzuführen ist. Der Startwert dieses Koinzidenzspektrums ist unmittelbar mit $W(\theta)$ verknüpft.

Ein auf den Kern wirkendes Feld kann nun diesen rein exponentiellen Zerfall bei konstantem $W(\theta)$ durch eine Aufhebung der Entartung der $m$-Unterzustände des Zwischenniveaus stören. Die Entartung kann dabei sowohl durch ein lokales Magnetfeld als auch durch den elektrischen Feldgradienten aufgehoben werden. Ersteres koppelt an das magnetische Moment des Zwischenniveaus, letzterer an das Quadrupolmoment $Q$. In der Beschreibung der Winkelkorrelation müssen dann Interferenzen in der zeitlichen Entwicklung der $m$-Unterzustände berücksichtigt werden, womit sich die vorher konstante Winkelkorrelation zeitabhängig ändert und der exponentielle Verlauf mit einer Störung moduliert wird. ${ }^{a}$

Für den hier relevanten Fall einer statischen elektrischen Quadrupolwechselwirkung in polykristallinen Materialien [43] lässt sich diese zeitabhängige Winkelkorrelation als

$$
W(\theta, t)=\sum_{k} A_{k k} G_{k k}(t) P_{k}(\cos \theta)
$$

\footnotetext{
${ }^{a}$ Die Interferenzterme führen dazu, dass auch Anisotropiekoeffizienten $A_{k_{1} k_{2}}$ mit gemischten Indizes relevant werden. Dies wird hier der Übersichtlichkeit halber nicht weiter berücksichtigt, da diese Abhängigkeit in polykristallinen Materialien, wie sie in dieser Arbeit untersucht werden, wieder verschwindet, sobald über alle Orientierungen gemittelt wird [43].
} 
mit dem zeitabhängigen Störfaktor

$$
G_{k k}(t)=\sum_{n=0}^{n_{\max }} s_{k n}(\eta) \cos (\underbrace{g_{n}(\eta) \omega_{Q}}_{\omega_{n}} t)
$$

darstellen. Die Frequenz $\omega_{n}$ entspricht hierbei der Übergangsenergie zwischen den aufgespaltenen $m$-Unterzuständen, die im Falle der elektrischen Quadrupolwechselwirkung in Einheiten der Quadrupolfrequenz

$$
\omega_{Q}=\frac{e Q V_{z z}}{4 I(2 I-1) \hbar},
$$

also einer zur größten Komponente des Feldgradienten im Hauptachsensystem, $V_{z z}$, proportionalen Größe, angegeben wird (siehe Kapitel 2.2). Eine in diesem Zusammenhang häufig auftretende, alternative Darstellung des Produkts aus Feldgradient und zugehörigem Moment bildet die Quadrupolkopplungskonstante

$$
v_{Q}=e Q V_{z z} / h .
$$

Der Spin des Zwischenniveaus legt die Anzahl der möglichen Übergangsfrequenzen $n_{\max }$ fest, wobei sich für $n=0$ der sogenannte hard-core, eine konstante Verschiebung des Störfaktors, ergibt. Jede Frequenz trägt mit einer bestimmten Amplitude $s_{k n}$ zur Störung bei. Die Abhängigkeit des Frequenzfakors $g_{n}$ und der Amplitude $s_{k n}$ vom Asymmetrieparameter $\eta$ (siehe Kapitel 2.2) werden nachfolgend am Beispiel der hier verwendeten ${ }^{111} \mathrm{In} /{ }^{111} \mathrm{Cd}$ Sonde diskutiert.

\subsubsection{Die ${ }^{111} \operatorname{In} /{ }^{111} \mathrm{Cd}-S o n d e$}

Die Anforderungen an eine PAC-Sonde resultieren unmittelbar aus der im vorherigen Kapitel beschriebenen Winkelkorrelation $W(\theta, t)$. Im Allgemeinen ist es hilfreich, eine möglichst große Amplitude der Störung vorliegen zu haben, womit die Anisotropiekoeffizienten der $\gamma$ - $\gamma$-Kaskade im Idealfall selbst möglichst groß sein sollten. Die Periode der Störung sollte nicht wesentlich kleiner als die Lebensdauer des Zwischenniveaus sein, um innerhalb des durch diese Lebensdauer zeitlich beschränkten PAC-Spektrums eine möglichst aussagekräftige Modulation beobachten zu können. Gleichzeitig darf diese Periode nicht die Zeitauflösung des Spektrometers unterschreiten. Da diese Zeitauflösung bei aktuellen PAC-Spektrometern bereits unter einer Nanosekunde liegt [147, 148], sind Zwischenniveaus mit größtmöglichen Momenten meist von Vorteil. Die Suche nach geeigneten PACIsotopen ist nach wie vor Gegenstand aktueller Forschung [149]; allein die Kenntnis über die Existenz einer Zefallskaskade genügt nicht, vielmehr müssen Momente und Anisotropien aufwendig bestimmt werden.

Bei allen PAC-Messungen wird die lokale Umgebung der PAC-Sonde, also der Gitterplatz, durch die chemischen Eigenschaften des Mutterisotops bestimmt. Das radioaktive Mutterisotop kann hierbei schon während der Synthese hinzugefügt oder nachträglich über Ionenimplantation oder Diffusion in ein schon synthetisiertes Material eingebracht werden. Alternativ kann das Mutterisotop über Neutroneneinfang direkt im Material erzeugt 


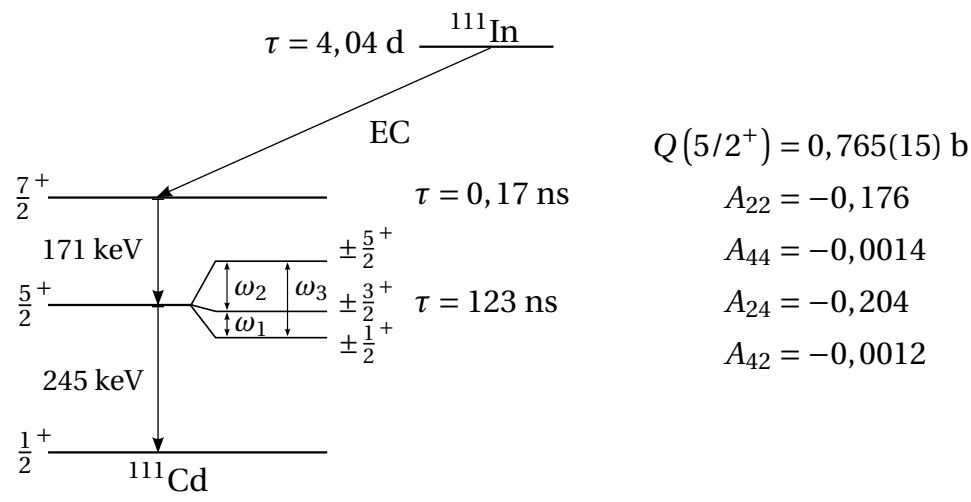

Abbildung 3.1: Zerfallsschema mit relevanten Lebensdauern, Spinzuständen und Übergangsenergien der ${ }^{111} \mathrm{In} / \mathrm{Cd}$-Sonde [152]. Die für die PAC-Messung notwendige $\gamma$ - $\gamma$-Kaskade wird durch einen Elektroneneinfang (EC) eingeleitet. Für das relevante Zwischenniveau sind zusätzlich das Quadrupolmoment [120] und die mit den Tabellen von Ferentz und Rosenzweig [145] berechneten Anisotropiekoeffizienten angegeben. Die im Störfaktor der elektrischen Quadrupolwechselwirkung auftretenden Wechselwirkungsfrequenzen $\omega_{n}$ entsprechen den Übergangsenergien zwischen den $m$ Unterzuständen des Zwischenniveaus.

werden. Die eigentliche PAC-Messung findet aber in jedem Falle am Tochterisotop statt, das zwar im Allgemeinen innerhalb der Zerfallskaskade nicht mehr den durch das Mutterisotop vorgegebenen Gitterplatz wechselt, jedoch die elektronische Struktur gemäß seiner Kernladungszahl verändert. Die Veränderung der elektronischen Struktur ist in der Regel bereits vor Erreichen des Zwischenniveaus abgeschlossen. Eine Ausnahme bildet hierbei der gelegentlich in isolierenden Proben auftretende, sogenannte after-effect, bei dem sich diese Struktur noch während der Messung verändert [150, 151].

Alle in dieser Arbeit durchgeführten PAC-Messungen nutzen die durch Ionenimplantation eingebrachte ${ }^{111} \mathrm{In} /{ }^{111} \mathrm{Cd}$-Sonde (siehe Abbildung 3.1). Diese Sonde genießt eine große Popularität, da sie alle oben genannten Anforderungen erfüllt und gleichzeitig kommerziell in Form von Indiumchlorid-Lösung (Mallinckrodt Deutschland $\mathrm{GmbH}$ ) verfügbar ist. Das erste Photon der $\gamma$ - $\gamma$-Kaskade weist eine Energie von $171 \mathrm{keV}$ auf, das zweite eine von $245 \mathrm{keV}$. Der Spin des Zwischenniveaus beträgt 5/2, womit sich bei rein elektrischer Quadrupolwechselwirkung pro Sondenumgebung bis zu drei von null verschiedene Frequenzen im PAC-Spektrum ergeben. Gleichung 3.3 vereinfacht sich zu

$$
W(\theta, t) \approx 1+A_{22} G_{22}(t) P_{2}(\cos \theta),
$$

mit

$$
G_{22}(t)=\sum_{n=0}^{3} s_{2 n}(\eta) \cos \left(g_{n}(\eta) \omega_{Q} t\right)
$$

da für die Anisotropiekoeffizienten $A_{44} / A_{22}<0,8 \%$ gilt.

Die für den Spin von 5/2 auftretenden Frequenzfaktoren $g_{n}(\eta)$ und Amplituden $s_{2 n}(\eta)$ wurden von Kajfosz [153] und Butz [154] in analytischer Form bestimmt. Die Faktoren $g_{n}$ 

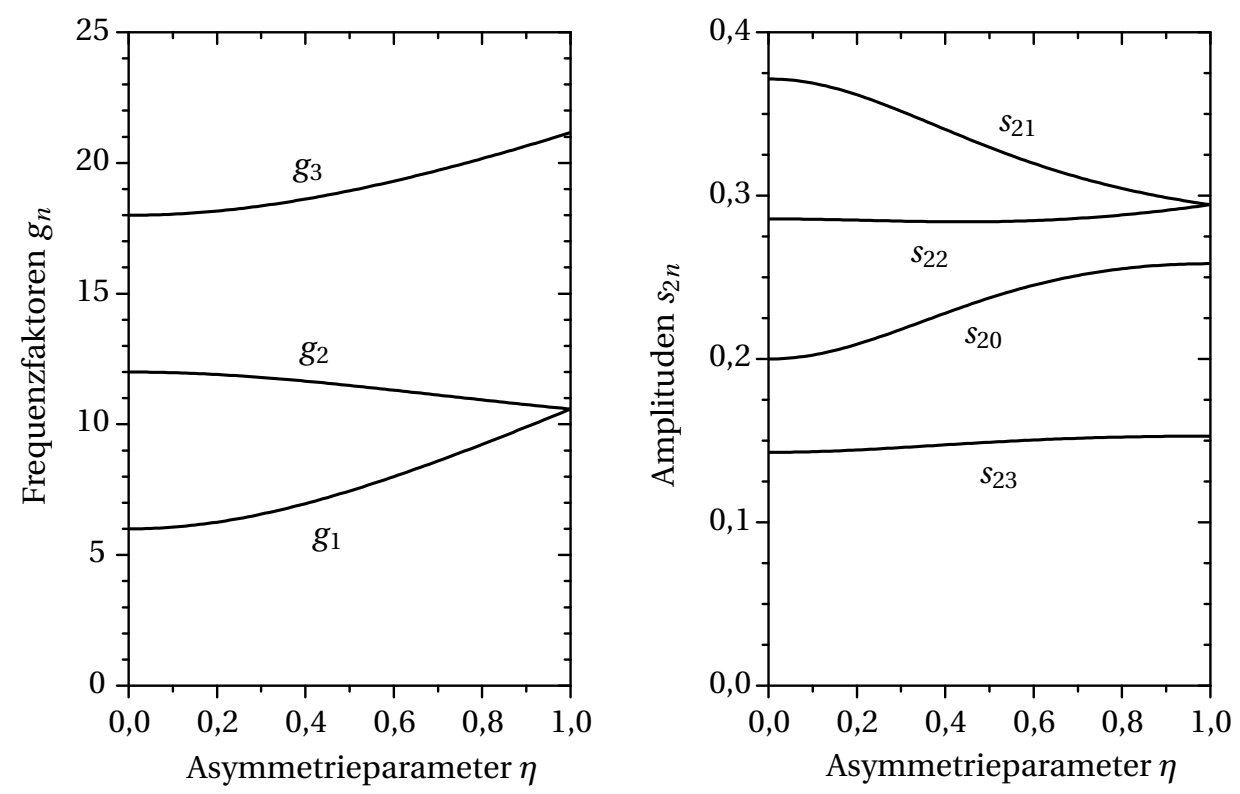

Abbildung 3.2: Frequenzfaktoren $g_{n}$ und Amplituden $s_{2 n}$ als Funktion des Asymmetrieparameters $\eta$ für einen Spin 5/2-Zustand im polykristallinen Mittel gemäß Referenzen [153, 154].

ergeben sich aus den Übergangsfrequenzen $\omega_{n}=g_{n} \cdot \omega_{Q}$, die aus den Differenzen der Energieeigenwerte des Hamiltonoperators der Quadrupolwechselwirkung folgen. Für gegebenen Spin hängen diese Eigenwerte lediglich von Quadrupolfrequenz und Asymmetrieparameter ab. Alle Zustände mit gleichem Betrag der magnetischen Quantenzahl sind entartet. Die zugehörigen Energieeigenwerte lauten:

$$
\begin{aligned}
& E_{ \pm \frac{5}{2}}=+\rho \cdot \cos \left(\frac{\varphi}{3}\right) \hbar \omega_{Q}, \\
& E_{ \pm \frac{3}{2}}=-\rho \cdot \cos \left(\frac{\varphi+\pi}{3}\right) \hbar \omega_{Q}, \quad \text { mit } \\
& E_{ \pm \frac{1}{2}}=-\rho \cdot \cos \left(\frac{\varphi-\pi}{3}\right) \hbar \omega_{Q}, \\
& \begin{array}{l}
\rho=2 \sqrt{28\left(1+\frac{\eta^{2}}{3}\right)}, \\
\varphi=\arccos \frac{80\left(1-\eta^{2}\right)}{\left[28\left(1+\frac{\eta^{2}}{3}\right)\right]^{\frac{3}{2}}} .
\end{array}
\end{aligned}
$$

Für die analytische Form von $s_{2 n}(\eta)$ sei auf Referenzen $[153,154]$ verwiesen. Der Verlauf beider Koeffizienten ist als Funktion des Asymmetrieparameters im polykristallinen Mittel in Abbildung 3.2 gezeigt. Beim Übergang hin zu Einkristallen oder texturierten Proben verliert der gezeigte Verlauf der Amplituden seine Gültigkeit. Wie oben schon angedeutet, müssen in diesem Falle gemischte Terme der Anisotropiekoeffizienten und die Orientierung der Detektoren relativ zur kristallographischen Achse berücksichtigt werden. Beide Aspekte führen effektiv zu einer Änderung der Amplituden $s_{2 n}$, die Übergangsfrequenzen $\omega_{n}$ bleiben erhalten [155]. 


\subsubsection{Messaufbau}

Die experimentelle Bestimmung der Störung $G_{22}(t)$ gelingt über die Störfunktion $R(t)=$ $A_{22}^{\text {eff }} G_{22}(t)$, die aus gemessenen Koinzidenzspektren $N(\theta, t)$ extrahiert werden kann. Die Messung dieser Koinzidenzspektren erfolgte mit Hilfe eines Messaufbaus, der aus vier Detektoren besteht. Alle Detektoren sind in einer Ebene um die Probe angeordnet, jeweils um $90^{\circ}$ zueinander versetzt positioniert und detektieren sowohl die 171-keV-Start- als auch die 245-keV-Stoppsignale der Kaskade. Hieraus ergeben sich insgesamt zwölf Koinzidenzspektren, davon acht unter einem Winkel $\theta$ von $90^{\circ}$ und vier bei $180^{\circ}$. Einsetzen dieser Winkel vereinfacht die Legendre-Polynome der Winkelkorrelation und somit auch die Bestimmung obiger Störfunktion.

Zur korrekten Zuordnung von Start- und Stoppsignalen wird neben einer guten Zeitauflösung auch eine ausreichende Energieauflösung der Detektoren benötigt. Einen hinreichenden Kompromiss bieten hierbei Szintillationsdetektoren, die mit einen mit Thallium dotierten Natriumiodid-Einkristall ausgestattet sind. In das Szintillatormaterial eintretende, ionisierende Strahlung erzeugt hierbei durch Sekundärprozesse Photonen mit einer Wellenlänge um $410 \mathrm{~nm}$ [156], die in einem angebrachten Photomultiplier in ein zur Primärenergie proportionales, elektrisches Signal umgewandelt werden. Die Auswertung dieses Signals erfolgt in zwei unabhängigen Zweigen: Einem schnellen, nur die Anstiegsflanke auswertenden Zweig zur Zeitbestimmung und einem langsameren, da über das gesamte Signal integrierenden, Zweig zur Energiebestimmung. Diese Unterscheidung gibt dem Aufbau den Namen slow-fast-Anordnung. Die Zeitauflösung der gesamten hier genutzten Apparatur liegt im Bereich von 3,5 ns [157], die Energieauflösung um 6\% ${ }^{a}$.

Das Schaltbild dieser slow-fast-Apparatur ist in Abbildung 3.3 gezeigt. Die Zuordnung eines Detektorsignals zu Start- oder Stoppsignalen erfolgt mit zwei Einkanaldiskriminatoren (engl. single channel analyzer, SCA), deren Amplitudenfenster entsprechend eingestellt werden. Der Zeitpunkt des Signals wird mit einem Constant-Fraction-Diskriminator (CFD) amplitudenunabhängig bestimmt. Die so identifizierten Startsignale starten einen Zeit-zu-Amplituden-Konverter (engl. time to amplitude converter, TAC), der vom nächsten eintreffenden Stoppsignal angehalten wird. Das ausgegebene Signal ist proportional zur gesuchten Dauer $t$ im Koinzidenzspektrum, die zugehörige Detektorkombination wird in der Routing-Einheit bestimmt. Auf diese Weise ergeben sich insgesamt zwölf Koinzidenzspektren $N(\theta, t)$, die über das Programm THOPAC [158] eingelesen und gespeichert werden.

Die Weiterverarbeitung der Koinzidenzspektren hin zur Störfunktion erfolgt mit dem Programm SpectraPac in Version 1.94 [147]. Hierbei werden die den Untergrund bildenden Zufallskoinzidenzen abgezogen sowie alle Spektren auf einen gemeinsamen Nullpunkt verschoben. Der Untergrund bestimmt sich aus einem exponentiellen Fit im hinteren Teil des Spektrums; der Nullpunkt aus einem vollständigen Fit der Messdaten mit einer gaußgefalteten Exponentialfunktion, welcher der endlichen Zeitauflösung der Apparatur Rechnung trägt. Die so korrigierten Koinzidenzspektren werden jeweils für die Winkel $90^{\circ}$ und $180^{\circ}$

${ }^{a}$ Gemessene Energieauflösung der 1173-keV-Linie von ${ }^{60} \mathrm{Co}$. 


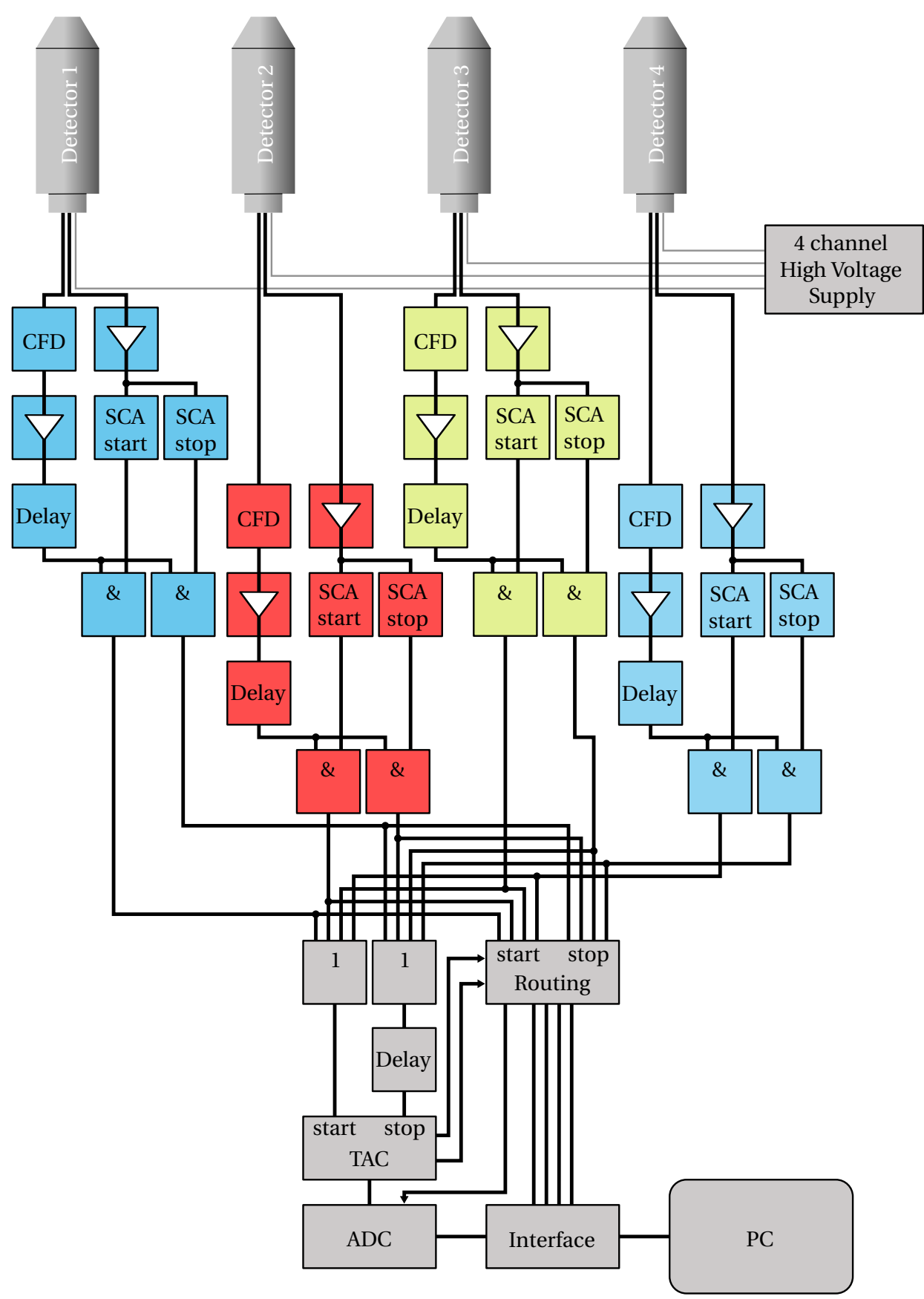

Abbildung 3.3: Skizze der aus 4 Detektoren bestehenden slow-fast PAC-Apparatur. Der Zeitpunkt eines $\gamma$-Zerfalls wird im CFD aus dem Anodensignal bestimmt. Das Dynodensignal wird verstärkt (Dreieck) und im SCA nach Start- bzw. Stoppsignal diskriminiert. Bei gleichzeitigem Eintreffen (\&) starten bzw. stoppen sie den TAC. Zusammen mit der Routingeinheit und dem ADC können anschließend die koinzidenten Signale dem Spektrum der jeweiligen Detektorkombination zugeordnet und gespeichert werden (mit freundlicher Genehmigung von M. Nagl [148]). 
geometrisch gemittelt, anschließend kann hieraus die Störfunktion gemäß

$$
R(t)=2 \frac{\bar{N}\left(180^{\circ}, t\right)-\bar{N}\left(90^{\circ}, t\right)}{\bar{N}\left(180^{\circ}, t\right)+2 \bar{N}\left(90^{\circ}, t\right)} \approx A_{22}^{\mathrm{eff}} G_{22}(t)
$$

durch Einsetzten der Legendre-Polynome gebildet werden [159]. Der in den Koinzidenzspektren enthaltene exponentielle Abfall der Messdaten kürzt sich heraus. Statt des Anisotropiekoeffizienten $A_{22}$ muss ein effektives $A_{22}^{\text {eff }}$ zur Skalierung verwendet werden, da die endliche Ausdehnung der Detektoren die Amplitude der Störung vermindert.

Die Störfunktion $R(t)$ enthält alle Informationen der PAC-Messung. Die eigentliche Auswertung und Interpretation der Messdaten erfolgt anhand von Modellen, die diese Störfunktion reproduzieren sollen. Dies wird im nachfolgenden Kapitel näher beschrieben.

Der oben beschriebene Messaufbau steht in dreifacher Ausführung zur Verfügung. In Zweien lässt sich die Temperatur während der Messung verändern, in der dritten kann eine mechanische Spannung auf die Probe einwirken. Der jeweilige Parameter wird über die Dauer einer Messung, die ungefähr einen Tag beträgt, konstant gehalten. Tiefe Temperaturen werden mit einem Helium-Verdampferkryostat (RGD 510, Leybold-Heraeus) erreicht, hohe Temperaturen in einem Rohrofen [160]. Um einer etwaigen Oxidation bei hohen Temperaturen vorzubeugen, befinden sich die Proben während der Messung in einer evakuierten Quarzampulle (siehe Kapitel 3.2). Die mechanische Belastung erfolgt mittels einer pneumatischen Presse (PFW 056, TOX-Pressotechnik) in einer Geometrie, in der die Kraft senkrecht zur Detektorebene auf die Probe wirkt. Zur Kraftübertragung werden Stempel aus gehärtetem Stahl verwendet, wobei die Kraftmessung mit einem Kraftsensor erfolgt, der seriell in die Lastachse eingebaut ist. Die auf die Probe wirkende Spannung wird über das Verhältnis aus Kraft und Probenquerschnitt berechnet. Konstruktionsbedingt erfolgt während der Kompression keine Dehnungsmessung, jedoch kann unter der Annahme der Volumenerhaltung die plastische Dehnung einer Probe über den veränderten Probenquerschnitt nach einer Belastung abgeschätzt werden.

\subsubsection{Datenauswertung}

Die bisherige Beschreibung beschränkt sich auf eine Situation, in der alle zum Messspektrum beitragenden Sonden einem identischen Feldgradienten ausgesetzt sind. Für diesen Fall könnte die gemessene Störfunktion $R(t)$ unmittelbar mit Gleichung 3.8 angepasst und die Wechselwirkungsparameter extrahiert werden. Diese Situation wird im Allgemeinen nicht beobachtet, vielmehr treten unterschiedlichste Sondenumgebungen auf, die alle zur experimentellen Störfunktion beitragen. Es ergibt sich eine für das jeweilige Probenstück charakteristische Feldgradientenverteilung.

Sonden, die beispielsweise in unterschiedliche Phasen eingebaut $[47,138]$ oder an unterschiedliche Punktdefekte angelagert [143, 144] sind, tragen auch mit unterschiedlichen Quadrupolfrequenzen und Asymmetrieparametern zum Spektrum bei. Diesem Aspekt kann durch eine Summe der Störfunktionen unterschiedlicher Umgebungen Rechnung getragen werden. Die einzelnen Umgebungen tragen entsprechend ihres Anteils $f_{i}$ zum Spektrum bei. Eine Darstellung der Fouriertransformation der Störfunktion, $A(\omega)$, erleichtert hierbei unter Umständen die Zuordnung einzelner Anteile. 
Darüber hinaus gibt es eine Vielzahl von Mechanismen, die den charakteristischen EFG einer Phase nur minimal ändern. Die vorher scharf begrenzte Verteilung des Feldgradienten der Komponente $f_{i}$ weist dadurch eine gewisse Breite auf. Potentielle Quellen dieser Verbreiterung sind beispielsweise entfernte Punkt- und Liniendefekte, die durch ihr Dehnungsfeld den EFG beeinflussen. Eine quantitative Untersuchung von Verteilungen dieser Art blieb bisher aus. Ungeachtet der wahren Natur dieser Verteilungen können aber die meisten PAC-Spektren unter der Annahme einer Lorentz- oder Gauß-Verteilung der größten Komponente des Feldgradienten, $V_{z z}$, hinreichend gut beschrieben werden. Es lässt sich zeigen, dass solche Verteilungen im Frequenzraum zu einer exponentiellen bzw. gaußförmigen Dämpfung der Störfunktion im Zeitraum führen [161]. Diese Aussage ist nicht nur auf obige Spezialfälle beschränkt, vielmehr führt jede endliche Verbreiterung eines scharfen Feldgradienten zu einer zeitlichen Dephasierung der Störung im Zeitraum. Aus dieser Erkenntnis ergibt sich eine weitverbreitete Modifikation von Gleichung 3.8, die eine relative Unschärfe $\delta$ der Quadrupolfrequenz berücksichtigt [162, 163]:

$$
G_{22}(t)=\sum_{i} f_{i} \sum_{n=0}^{3} s_{2 n}\left(\eta_{i}\right) \cdot \cos \left(g_{n}\left(\eta_{i}\right) \omega_{Q i} t\right) \cdot \exp \left(-\frac{1}{a}\left(g_{n}\left(\eta_{i}\right) \omega_{Q i} \delta_{i} t\right)^{a}\right)
$$

Für Lorentz-Verteilungen gilt $a=1$, für Gauß-Verteilungen $a=2$. Die Verteilungsbreite $\delta$ entspricht der Halbwertsbreite der Lorentz-Verteilung bzw. der Standarabweichung der Gauß-Verteilung. Ein variabler Parameter $a$ ist als Verallgemeinerung der Verteilungsform denkbar, wird aber üblicherweise nicht benutzt.

Die Verwendung dieser Gleichung birgt bei größeren Verteilungsbreiten allerdings erhebliche Gefahren der Fehlinterpretation, deren Auswirkungen zuerst von Forker [164] quantifiziert wurden. Die Problematik besteht darin, dass die mit einer Verteilung von $V_{z z}$ im Allgemeinen einhergehende Verteilung von $\eta$ ignoriert wird. Dies - in Kombination mit der Tatsache, dass die Eigenwerte des Hamiltonoperators der Quadrupolwechselwirkung abhängig von $\eta^{2}$ sind, der experimentelle Zugang zum Asymmetrieparameter sich also auf dessen Betrag beschränkt - führt zu einer von der Verteilungsbreite abhängigen, systematischen Fehleinschätzung der Mittelwerte der Quadrupolfrequenz und des Asymmetrieparameters. Für den Spezialfall eines eigentlich axialsymmetrischen Feldgradienten wird diese Abweichung extremal, sowohl $\eta$ als auch $\omega_{Q}$ werden durch einen solchen Fit maximal überschätzt.

Eine Möglichkeit die Zuordnung insbesondere von $\eta$ zu verbessern, besteht in der von Butz [165] vorgeschlagen Kreuzkorrelation experimenteller Störfunktionen mit Gleichung 3.8. Die Darstellung dieser Kreuzkorrelation in beispielsweise dem Czjzek-Diagramm ${ }^{a}$

\footnotetext{
${ }^{a}$ Das Czjzek-Diagramm [166] bietet eine lineare Darstellung des $V_{z z}-\eta$-Parameterraums in der Linien konstanter Feldgradientenstärke oder konstanten Asymmetrieparameters als Geraden auftauchen, der Übergang bei $\eta=1$ aber gleichzeitig stetig ist. Dies ist in einer einfachen kartesischen Darstellung von $V_{z z}$ und $\eta$ nicht der Fall, da hier beim Übergang, durch den Tausch von $V_{z z}$ und $V_{y y}$ in der Definition von $\eta$, der Parameterraum in einem anderen Quadranten fortgesetzt wird. Die Darstellung erfolgt in einem kartesischen Koordinatensystem anhand der Ersatzgrößen $Y=-2 V_{x x}$ und $X=2\left(2 V_{z z}+V_{x x}\right) / \sqrt{3}$. Es ergibt sich eine Darstellung mit sechszähliger Symmetrie, von der nur der Teil benötigt wird, in dem $V_{z z}$ die betragsmäßig größte Komponente darstellt. Die Skalierung der Achsen kann nach Belieben so gewählt werden, dass nicht $V_{z z}-\eta$ sondern $\omega_{Q}-\eta$ abgebildet werden. Eine Darstellung des reduzierten Czjzek-Diagramms findet sich in Abbildung 3.4c. An der oberen
} 


\section{Kapitel 3 Experimentelle Methoden}

(siehe Abbildung 3.4c) soll es ermöglichen, anhand der maximalen Korrelation im $\omega_{Q^{-}}$ $\eta$-Parameterraum den korrekten Asymmetrieparameter zu identifizieren. Da die Art der Darstellung allein allerdings noch nichts an der Tatsache ändert, dass nur der Betrag von $\eta$ gemessen werden kann, lohnt es sich zur Klassifizierung von Feldgradientenverteilungen den in negative $\eta$ fortgesetzten Parameterraum vorzustellen [167]. So sollten insbesondere Situationen, in denen eine wahre Abweichung von $\eta=0$ vorliegt, von Situationen unterschieden werden, in denen lediglich eine symmetrische Verteilung um $\eta=0$ vorliegt, die Axialsymmetrie also im Prinzip erhalten bleibt. Der Fall einer wahren Abweichung von der Axialsymmetrie wäre beispielsweise durch den intrinsischen EFG eines orthorhombischen Kristalls oder durch den Symmetriebruch eines axialsymmetrischen EFGs eines uniform belasteten Einkristalls gegeben. Im Czjzek-Diagramm würde dann der Bereich maximaler Korrelation bei $\eta \neq 0$ liegen, ein Spiegelbild der umgebenden Verteilung bei negativen $\eta$ per Definition der Kristallachsen nicht existieren. Im Gegensatz dazu stünden Situationen wie das oben genannte Beispiel, in denen Defekte im Material, die in regelloser Orientierung zu den Kristallachsen stehen, eine Verteilung von $\eta$ um null erzeugen. Hierbei läge das Maximum der Korrelation bei $\eta=0$ und wäre von einer konvexen Verteilung, die sich symmetrisch auch zu negativen $\eta$ ausbildet, umgeben.

Eine weitere Möglichkeit der Datenanalyse besteht in der Simulation der Störfunktion bei vorgegebener Feldgradientenverteilung. Diese Variante verlangt das meiste Vorwissen über die beteiligte Verteilung und kommt in der Literatur vermutlich auch deshalb eher selten zum Einsatz. Beispiele dieses Ansatzes finden sich in Form zweier Studien von zufällig verteilten Punktladungen als Quelle der Verbreiterung [168, 169]. In einer der Studien werden simulierte Störfunktionen reproduziert, ein Vergleich zu experimentellen Daten fehlt. Die andere Studie beschränkt sich beim Vergleich mit experimentellen Daten auf die qualitative Form der Störfunktion, was einem verallgemeinerten Fit-Parameter gleich kommt. Ein quantitativer Vergleich zwischen gemessener Verteilungsbreite und deren Ursprung blieb in beiden Fällen aus.

In der vorliegenden Arbeit wird zur Analyse der Messdaten eine Kombination der beiden erstgenannten, etablierteren Ansätze verfolgt. Experimentelle Störfunktionen werden mit Gleichung 3.11 angepasst sowie die dadurch erhaltenen Parameter $\omega_{Q}, \eta$ und $\delta$ diskutiert. Zu diesem Zwecke wurde ein Python-Programm erstellt, das eine Stapelverarbeitung dieser Anpassung ermöglicht. Der Ansatz der Kreuzkorrelation wird genutzt, um die EFGVerteilungen zu klassifizieren. Hierfür wird das Programm Winfit von F. Heinrich [170] in Version 3.0.4 verwendet.

Eine für diese Arbeit typische Störfunktion ist in Abbildung 3.4 dargestellt. Es handelt sich dabei um ein bei Raumtemperatur gemessenes PAC-Spektrum der auf dem Al-Platz in $\mathrm{Ti}_{2} \mathrm{AlN}$ substitutionell eingebauten Cadmiumsonde. Die ${ }^{111} \mathrm{In} /{ }^{111} \mathrm{Cd}$-Sonde wurde mittels Ionenimplantation (siehe Kapitel 3.2) in das Material eingebracht, die dadurch entstanden Implantationsschäden durch Ausheilen der Probe für fünf Stunden bei $900^{\circ} \mathrm{C}$ gemäß Referenz [47] minimiert. Zur besseren Übersicht über auftretende Wechselwirkungsfrequenzen ist neben der Störfunktion auch deren Fouriertransformation abgebildet. In beiden

Kante des Parameterraums würde der Bereich negativer $\eta$ angrenzen, an der Unterkante besagter Übergang stattfinden. 
(a)

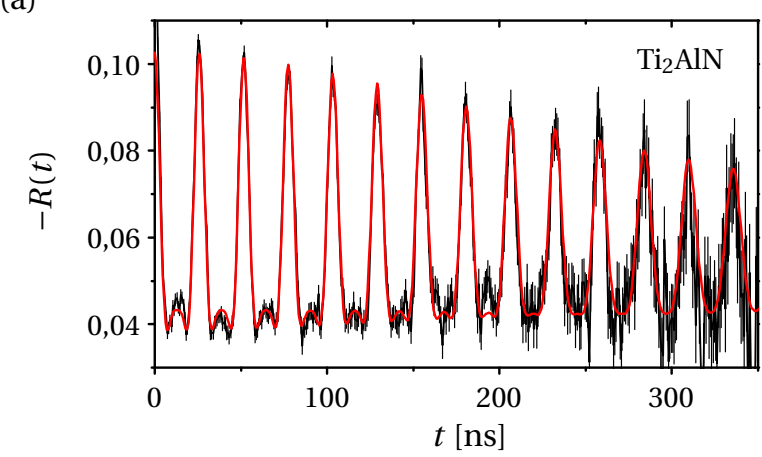

(c)

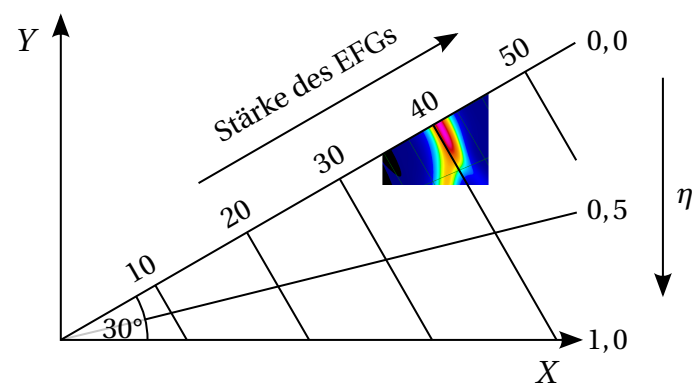

(b)

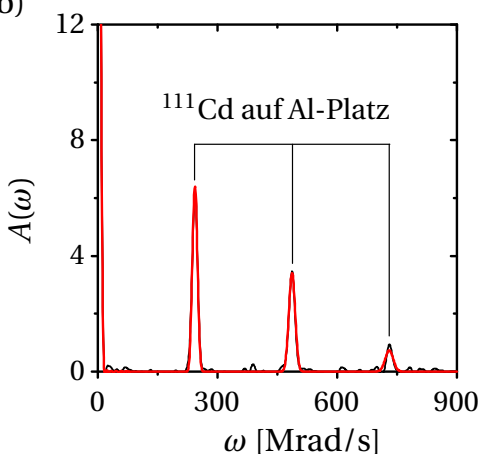

(d)

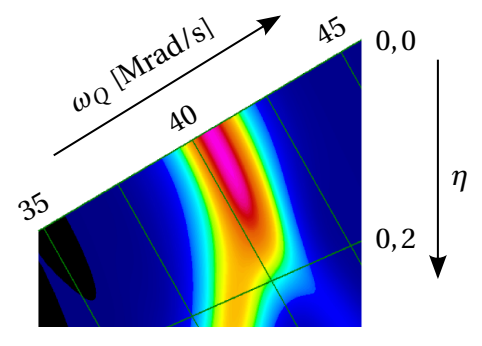

Abbildung 3.4: Beispiel eines PAC-Spektrums an der Cd-Sonde in $\mathrm{Ti}_{2} \mathrm{AlN}$. Die aus Gleichung 3.10 experimentell ermittelte Störfunktion $R(t)$ wird mit Gleichung 3.11 angepasst (a). Die Fouriertransformation beider Kurven zeigt das für einen axialsymmetrischen Feldgradienten typische Frequenztriplett (b). Verbreiterungen der EFGTensorkomponenten gehen mit einer Dämpfung der Störfunktion einher. Im CzjzekDiagramm wird die Kreuzkorrelation zwischen experimentellen Daten und idealer Störfunktion (Gleichung 3.8) dargestellt (c,d). Die Stärke des EFGs kann hierbei direkt über $V_{z z}$, die Quadrupolkopplungskonstante $v_{Q}$ oder die Quadrupolfrequenz $\omega_{Q}$ angegeben werden. Die maximale Intensität der Korrelation (lila) gibt einen Hinweis auf die wahre Struktur des EFGs, insbesondere auf $\eta$.

Abbildungen ist in rot der Fit nach Gleichung 3.11 eingezeichnet. Das zugehörige CzjzekDiagramm der Kreuzkorrelation ist im unteren Teil in reduzierter Form dargestellt. Der gezeigte Ausschnitt der Korrelation beschränkt sich auf die in der Messung auftretenden Quadrupolfrequenzen und Asymmetrieparameter. Die Korrelation erscheint länglich, da sich Gleichung 3.11 aufgrund der $\eta^{2}$-Abhängigkeit um $\eta=0$, dem Maximum der Korrelation, kaum ändert.

\subsection{Ionenimplantation}

Das für die PAC-Messungen benötigte radioaktive Isotop ${ }^{111}$ In wurde in dieser Arbeit ausnahmslos durch Ionenimplantation in die untersuchten Materialien eingebracht. Die Implantation erfolgte mit dem Göttinger Cockcroft-Walton-Beschleuniger IONAS [171] ausgehend von einer $300 \mu \mathrm{l}$ Indiumchlorid-Lösung (Mallinckrodt Deutschland GmbH) mit einer Aktivität von 111 MBq. Eine detaillierte Beschreibung des gesamten Verfahrens findet 


\section{Kapitel 3 Experimentelle Methoden}

sich bei Uhrmacher et al. [172].

Da das anfängliche Volumen der Lösung für eine sinnvolle Handhabung zu groß ist, lohnt es sich, die Lösung in einem Teflongefäß unter einer Infrarot-Lampe auf ca. $60 \mu \mathrm{l}$ einzutrocknen. Dieses Restvolumen wird sukzessive in Form von $5 \mu$ l-Tropfen in das Reservoir der Quellenstäbchen pipettiert und eingetrocknet. Im Quellenbereich des Beschleunigers eingebracht, sublimiert von diesen Stäbchen im Betrieb das zurückbleibende Indiumsalz und reichert das Quellenplasma mit ${ }^{111} \mathrm{In}^{+}$-Ionen an. Mit Hilfe einer Extraktionsspannung von $30 \mathrm{kV}$ werden Ionen aus dem Plasma extrahiert, die mittels eines Sektormagneten nach Masse selektiert werden. Eine weitere Beschleunigung der Ionen bei $370 \mathrm{kV}$ ergibt eine für die Implantation zur Verfügung stehende Gesamtenergie von $400 \mathrm{keV}$. Pro Implantation werden zwischen $10^{12}$ und $10^{13}$ Indiumionen auf einer Fläche von $10 \mathrm{~mm}^{2}$ in die Proben, die auf einem leitfähigen Halter aufgeklebt sind, eingebracht.

Die auf die Probe treffenden Ionen wechselwirken sowohl mit den Elektronen als auch mit den Kernen der im Material vorhanden Atome. Erstere Wechselwirkung wird dabei als elektronischer, letztere als nuklearer Energieverlust bezeichnet. Beide übertragen Energie vom Ion auf das Material und bremsen somit das Ion ab. Der elektronische Energieverlust dominiert bei hohen Energien und wird durch die Bethe-Formel beschrieben [173]. Der nukleare Energieverlust gewinnt mit abnehmender Ionenenergie an Bedeutung und ist maßgeblich für die Gitterschädigung verantwortlich, da hierbei Atome durch direkte Stöße von ihrer ursprünglichen Positionen verlagert werden. Beide Prozesse lassen sich mit dem Monte-Carlo-Simulationsprogramm SRIM2011 [174] beschreiben, um die Reichweite der Ionen und die Defektverteilung in den Materialien zu ermitteln.

Bei einer Energie von $400 \mathrm{keV}$ ergeben sich für die Indiumionen innerhalb der MAXPhasen Eindringtiefen, die mit einer Standardabweichung von $40 \mathrm{~nm}$ gaußförmig um einen Mittelwert von ungefähr $100 \mathrm{~nm}$ verteilt sind. Die dabei entstehenden Defekte sind ebenfalls gaußverteilt und führen, beispielsweise für $\mathrm{Ti}_{2} \mathrm{InC}$, innerhalb des Implantationsvolumens zu einem Anteil versetzter Atome von bis zu 25\%. Es ist jedoch davon auszugehen, dass einige der erzeugten Defekte schon während der Implantation ausheilen. Alle anderen Defekte werden im Anschluss an die Implantation bei Temperaturen von bis zu $1173 \mathrm{~K}$ ausgeheilt.

Für das Ausheilen kamen zwei verschiedene Verfahren zum Einsatz, die beide eine Oxidation der Probe nach Möglichkeit vermeiden sollten. Beim ersten Verfahren wurden die Proben in einer bei $10^{-5}$ mbar evakuierten Quarzampulle eingeschlossen, die in einem Muffelofen (L3/R, Nabertherm) auf die gewünschte Temperatur erhitzt wurde. Dieses Verfahren kam für alle MAX-Phasen und Zink zum Einsatz. Beim zweiten Verfahren wurden die Proben nicht in einer Quarzampulle, sondern direkt in einem Hochtemperatur-Ofen [148] erhitzt, der unter Vakuumbedingungen von bis zu $10^{-8}$ mbar arbeitet und es zulässt, Temperatur und Druck während des Heizens zu protokollieren. Dieses Verfahren kam lediglich bei Titan zum Einsatz, da hier die kurze Heizdauer von fünf Minuten sowie die Reaktivität des Titans besondere Anforderungen an Temperaturregelung und Vakuumbedingung stellten. Bei beiden Verfahren konnte der Anteil an radioaktiven Sonden, der das Material während des Ausheilens verlassen hatte, über eine Messung der Probenaktivität vor und nach dem Heizen bzw. durch eine Messung der Aktivität der leeren Quarzampulle abgeschätzt werden. 
Neben der Reduktion der Implantationsschäden werden beim Ausheilen die implantierten Sonden auch auf definierten Gitterplätzen im Material eingebaut, was es im Idealfall ermöglicht, ein eindeutiges PAC-Spektrum aufzuzeichnen. Welche Ausheilparameter dafür nötig sind, wird in Kapitel 5 individuell für die untersuchten Probensysteme erläutert.

\subsection{Röntgenbeugung}

Auf die Entdeckung der Beugung von Röntgenstrahlen an Kristallen von Friedrich, Knipping und Laue im Jahre 1912 [132] folgte von W. H. und W. L. Bragg 1913 [133] unmittelbar die Erfindung des Röntgendiffraktometers, mit dem es möglich wurde, Kristallstrukturen experimentell zu bestimmen - die Grundlagen der Röntgendiffraktometrie waren entwickelt. Über die letzten hundert Jahre hinweg haben sich hieraus neben der Verwendung in der eigentlichen Strukturbestimmung von Kristallen oder Molekülen [175] unter anderem Anwendungen in der Bestimmung der Kristallitgröße und interner Spannungen [176, 177] ergeben. Ausführliche Beschreibungen der Röntgendiffraktometrie (engl. $x$-ray diffraction, XRD) finden sich beispielsweise bei Warren [134] oder Massa [178]. In diesem Unterkapitel folgen eine Erklärung der Methode sowie eine Beschreibung der hier verwendeten Messaufbauten und des benutzten Auswerteverfahrens.

Zentrale Größen der Röntgendiffraktometrie sind die Bragg-Gleichung [133]

$$
2 d \sin \theta=n \lambda,
$$

die bei gegebener Wellenlänge $\lambda$ jedem Netzebenenabstand $d$ einen Reflex der Ordnung $n$ unter einem Streuwinkel $2 \theta$ zuweist und der Strukturfaktor $[134,178]$, der den jeweiligen Reflexen Intensitäten zuordnet. Die vorkommenden Netzebenenabstände und Strukturfaktoren ergeben sich aus Raumgruppe und Basis des Kristalls. Die experimentelle Bestimmung des Diffraktogramms lässt es somit zu, die Kristallstruktur zu bestimmen. Bei korrekter Zuordnung können alle Reflexe mit einem Miller'schen Index versehen werden.

Die hier verwendeten Diffraktometer sind das D8 Advance (Bruker AXS) in Göttingen und das SmartLab (Rigaku) in Philadelphia, die beide mit einer Kupferröhre in $\theta-2 \theta$ Streugeometrie betrieben wurden. Im Falle des D8 Advance wurde die $\mathrm{Cu} K_{\alpha}$-Strahlung mittels Göbelspiegel und Monochromator von den anderen Röntgenlinien isoliert, beim SmartLab kam hierfür ein Nickel-Filter zum Einsatz. Die Schrittweite beträgt in allen Spektren $0,02^{\circ}$, die Messdauer lag je nach Intensität bei bis zu $40 \mathrm{~s}$ pro Messpunkt.

Die Auswertung der Spektren der polykristallinen Proben erfolgte mit Hilfe der im Programm FullProf [179] implementierten Rietveld-Methode [180], bei der ein theoretisches Pulverspektrum an das experimentelle Diffraktogramm angepasst wird. Die Form der Reflexe wurde mit einem Pseudo-Voigt-Profil approximiert ${ }^{a}$. Variationen der Intensität wur-

\footnotetext{
${ }^{a}$ Prinzipiell lassen sich bei bekannter instrumenteller Auflösungsfunktion anhand der winkelabhängigen Verbreiterung der Reflexe Informationen über die Kristallitgröße des Materials und die darin enthaltenen interne Spannungen ableiten (siehe abschließende Diskussion in Kapitel 6.6). Wenngleich diese Auflösungsfunktion durch eine Messung eines Lanthanhexaborid-Pulver (NIST-Standard 660B [181]) für beide Spektrometer ermittelt werden konnte, genügte die Statistik der an den MAX-Phasen aufgenommen Spektren nicht, um brauchbare Parameter der internen Spannung zu extrahieren. Eine signifikante Reflexverbreiterung, die mit
} 
den gemäß eines Modells zugelassen, bei dem die Vorzugsrichtung der Körner durch prolate bzw. oblate Rotationsellipsoide approximiert werden ${ }^{a}$. Systematische Fehler in der Winkelangabe der Spektrometer wurden durch Verschiebungen des Spektrums ausgeglichen, was eine erheblich genauere Bestimmung der Gitterkonstanten ermöglicht. Zur Anpassung des Untergrundes wurde ein Polynom vierter Ordnung verwendet.

\subsection{Transmissionselektronenmikroskop}

Das erste Transmissionselektronenmikroskop (TEM) wurde 1932 von Knoll und Ruska [135] aufgebaut und bildete damit die Grundlage für ein äußerst vielseitiges Instrument der Materialforschung: Ein Gerät mit atomarer Auflösung, bei dem es gelingt per Knopfdruck zwischen Abbildungs- und Beugungsmodus umzuschalten. Statt Photonen werden zur Abbildung hochenergetische Elektronen eingesetzt, die es durch ihre kurze De-BroglieWellenlänge ermöglichen, die Auflösungsgrenze des konventionellen Lichtmikroskops um drei Größenordnungen zu unterschreiten [135]. Eine ausführliche Beschreibung der Transmissionselektronenmikroskopie findet sich bei Williams und Carter [136].

In dieser Arbeit wird das Philips CM12 zur Abbildung eines Querschnittes der Dünnschichtproben eingesetzt, das mit einer Lanthanhexaborid-Quelle und einer Beschleunigungsspannung von $80 \mathrm{keV}$ betrieben wird. Die verwendeten Abbildungsmodi umfassen die Hellfeldabbildung, bei der lediglich ungebeugte Elektronen zur Abbildung beitragen und dadurch der Kontrast selbiger verbessert wird, die Dunkelfeldabbildung, bei der sich die Abbildung aus Elektronen eines ausgewählten Beugungsreflexes zusammensetzt und es somit ermöglicht wird, Bereiche gleicher Kornorientierung zu identifizieren sowie die Feinbereichsbeugung, bei der ein Beugungsbild eines ausgesuchten Bereichs abgebildet wird.

Die Präparation der TEM-Lamelle erfolgte mit Hilfe eines Ionenmikroskops (engl. focused ion beam microscope, FIB), indem durch einen Galliumionenstrahl Bereiche der Schicht durch Sputtern entfernt wurden, bis nur noch die gewünschte Lamelle zurückblieb. Hierbei kam das Zweistrahl-Gerät Nova 600 NanoLab (FEI) zum Einsatz, in dem parallel zum Ionenmikroskop ein Rasterelektronenmikroskop betrieben wird. Noch vor dem eigentlichen Freilegen der Lamelle wurde das Dünnschichtsystem lokal mit zwei Platinschichten bedeckt, von der die erste durch den Elektronenstrahl und die zweite durch den Ionenstrahl aus einem sogenannten Precursor-Gas abgeschieden wurde. Die Lamelle wurde anschließend mit dem Ionenstrahl bei einer Energie von $30 \mathrm{keV}$ und Stromstärken zwischen $7 \mathrm{nA}$ und $30 \mathrm{pA}$ freigelegt und auf ungefähr $100 \mathrm{~nm}$ Dicke verjüngt. Die abschlieBende Behandlung der Lamellenoberfläche erfolgte bei einer Ionenenergie von $5 \mathrm{keV}$ und einer Stromstärke von 29 pA. Der Transport der Lamelle erfolgte innerhalb des Gerätes mit dem Nanomanipulator Omniprobe (Oxford Instruments), außerhalb mit einem Kupferhalter, an dem die Lamelle mit Platin befestigt wurde.

der Scherrer-Gleichung in Verbindung gebracht werden könnte, wurde erwartungsgemäß nicht beobachtet, da die Kristallitgrößen bis auf in der Dünnschichtprobe im Bereich einiger Mikrometer liegen.

${ }^{a}$ Eine solche Beschreibung genügt nicht zur Rekonstruktion der Orientierungsdichteverteilungsfunktion. 


\subsection{Synthese der MAX-Phasen}

Die untersuchten MAX-Phasen wurden selbst oder durch Kollaborationspartner ${ }^{a b c}$ an der Drexel University bzw. der Manchester Metropolitan University synthetisiert. Nachfolgend findet sich ein Beschreibung der Prozessparameter. Polykristallines Titan und Zink wurden in einer Reinheit von 99,99\% käuflich von Alfa Aesar erworben. Optische Mikroskopie an polierten und angeätzten Oberflächen zeigt, dass die Korngrößen der untersuchten Titanund Zink-Proben im Bereich von einigen dutzend bzw. einigen hundert Mikrometern liegen.

\subsubsection{Volumenmaterial}

Die grundlegenden Synthesebedingungen der untersuchten MAX-Phasen finden sich in einer ausführlichen Beschreibung in den Referenzen [34, 36, 182, 183]. Die Herstellung der von den Kollaborationspartnern erhaltenen Volumenproben weicht von diesen Angaben teilweise ab, die verwendeten Chargen stimmen mit denen aus Referenzen [34, 47, 182, 184] überein. $\left(\mathrm{Ti}_{0,5}, \mathrm{~V}_{0,5}\right)_{2} \mathrm{AlC}$ wurde nach der Beschreibung von Gupta et. al. [183] eigenhändig synthetisiert. Die Synthese von $\mathrm{Ti}_{2}\left(\mathrm{Al}_{0,5}, \mathrm{In}_{0,5}\right) \mathrm{C}$ stellt ein Novum dar.

Die Synthese der MAX-Phasen erfolgt im Allgemeinen ausgehend von elementaren Pulvern und Pellets, im Falle von stickstoffhaltigen MAX-Phasen auch ausgehend von Metallnitriden. Populäre MAX-Phasen wie $\mathrm{Ti}_{2} \mathrm{AlC}$ sind in Form von vorreagiertem Pulver kommerziell erhältlich. Das Ausgangsmaterial wird für einen halben Tag in einem Ofen bei ungefähr $400 \mathrm{~K}$ getrocknet und ähnlich lange in einer Kugelmühle vermischt. Anschließend wird das Pulver für das Sintern in der Heißpresse (HP) oder der heißisostatischen Presse (HIP) vorbereitet. In der Heißpresse wird das Pulver in einen Hohlzylinder aus Graphit gegeben, dessen Öffnungen mit beweglichen Zylindern verschlossen werden, die es erlauben, unter Vakuumbedingungen einen uniaxialen Druck auf das Pulver auszuüben und gleichzeitig das Pulver zu heizen. In der heißisostatischen Presse wird das Pulver in einer evakuierten Quarzampulle eingeschlossen, worin - sobald das Glas flüssig ist - das Pulver unter einer Argonatmosphäre isostatisch komprimiert und weiter aufgeheizt wird. In beiden Pressen werden etappenweise zunächst Temperatur und anschließend Druck erhöht, bis die gewünschten Prozessparameter für das Sintern erreicht sind, welche typischerweise für mehre Stunden gehalten werden. Eine detaillierte Beschreibung der Synthese folgt exemplarisch in Kapitel 5 anhand der neu entdeckten MAX-Phase $\operatorname{Ti}_{2}\left(\mathrm{Al}_{0,5}, \mathrm{In}_{0,5}\right) \mathrm{C}$. Eine Übersicht über die finalen Prozessparameter aller untersuchten MAX-Phasen ist in Tabelle 3.1 gegeben.

Alle auf diese Weise hergestellten Volumenproben sind überwiegend phasenrein (siehe Röntgendiffraktometriemessungen in Kapitel 5) und nahezu vollständig dicht. Optische Mikroskopie polierter und angeätzter Oberflächen zeigen Körner mit einem Durchmesser von einigen dutzend Mikrometern. Das Ätzen der Oberfläche erfolgte mit einer Lösung, die je zur Hälfte aus 10-prozentiger Flusssäure und 7-prozentiger Salpetersäure bestand.

\footnotetext{
${ }^{a} \mathrm{M}$. W. Barsoum, Department of Materials Science and Engineering, Drexel University, USA

${ }^{b}$ V. Vishnyakov, Dalton Research Institute, Manchester Metropolitan University, UK

${ }^{c}$ D. Jürgens, II. Physikalisches Institut, Universität Göttingen, Deutschland
} 
Kapitel 3 Experimentelle Methoden

Tabelle 3.1: Übersicht über die Prozessparameter der untersuchten MAX-Phasen. Die Systeme $\left(\mathrm{Ti}_{0,5}, \mathrm{~V}_{0,5}\right)_{2} \mathrm{AlC}$ und $\mathrm{Ti}_{2}\left(\mathrm{Al}_{0,5}, \mathrm{In}_{0,5}\right) \mathrm{C}$ wurden im Rahmen diese Arbeit synthetisiert, alle anderen MAX-Phasen wurden von den Kollaborationspartnern zur Verfügung gestellt (siehe Referenzen). Das Sintern erfolgte jeweils durch Heißpressen (HP) oder heißisostatischem Pressen (HIP) der Ausgangsmaterialien. Die angegebenen Prozessparameter geben den dabei maximal erreichten Druck und die maximal erreichte Temperatur an.

\begin{tabular}{|c|c|c|c|c|c|c|}
\hline & Ausgangsmaterial & Presse & Druck & Temperatur & Dauer & Ref. \\
\hline $\mathrm{Nb}_{2} \mathrm{AlC}$ & Nb-, Al-, C-Pulver & $\mathrm{HP}$ & $35 \mathrm{MPa}$ & $1873 \mathrm{~K}$ & $10 \mathrm{~h}$ & {$[47]$} \\
\hline $\mathrm{Ti}_{2} \mathrm{AlC}$ & $\mathrm{Ti}_{2} \mathrm{AlC}_{\text {-Pulver }}{ }^{a}$ & $\mathrm{HP}$ & $35 \mathrm{MPa}$ & $1573 \mathrm{~K}$ & $4 \mathrm{~h}$ & [47] \\
\hline $\mathrm{Ti}_{2} \mathrm{AlN}$ & Ti-, AlN-Pulver & HP & $35 \mathrm{MPa}$ & $1773 \mathrm{~K}$ & $4 \mathrm{~h}$ & {$[47]$} \\
\hline $\mathrm{Ti}_{2} \operatorname{InC}$ & Ti-, C-Pulver; In-Pellets & HIP & $50 \mathrm{MPa}$ & $1573 \mathrm{~K}$ & $7 \mathrm{~h}$ & [182] \\
\hline$\left(\mathrm{Ti}_{0,5}, \mathrm{Nb}_{0,5}\right)_{2} \mathrm{AlC}$ & Nb-, Ti-, $\mathrm{Al}_{4} \mathrm{C}_{3^{-}}, \mathrm{C}-\mathrm{Pulver}$ & HIP & $100 \mathrm{MPa}$ & $1873 \mathrm{~K}$ & $8 \mathrm{~h}$ & {$[34]$} \\
\hline$\left(\mathrm{Ti}_{0,5}, \mathrm{~V}_{0,5}\right)_{2} \mathrm{AlC}$ & Ti-, V-, Al-, C-Pulver & $\mathrm{HP}$ & $40 \mathrm{MPa}$ & $1873 \mathrm{~K}$ & $6 \mathrm{~h}$ & $-b$ \\
\hline $\mathrm{Ti}_{2}\left(\mathrm{Al}_{0,5}, \mathrm{In}_{0,5}\right) \mathrm{C}$ & Ti-, Al-, C-Pulver; In-Pellets & HP & $40 \mathrm{MPa}$ & $1573 \mathrm{~K}$ & $3 \mathrm{~h}$ & $-b$ \\
\hline $\mathrm{Ti}_{2} \mathrm{Al}\left(\mathrm{C}_{0,5}, \mathrm{~N}_{0,5}\right)$ & Ti-, Al-, AlN-, C-Pulver & HIP & $100 \mathrm{MPa}$ & $1673 \mathrm{~K}$ & $10 \mathrm{~h}$ & [184] \\
\hline
\end{tabular}

${ }^{a}$ Vorreagiertes Pulver von 3-One-2 LLC.

${ }^{b}$ Diese Arbeit.

Aus dem Volumenmaterial wurden mittels Funkenerosion ein Millimeter dicke Scheiben herausgeschnitten, die in Stücke mit $5 \times 6 \mathrm{~mm}^{2}$ großer Grundfläche unterteilt wurden. Die so entstandenen Oberflächen wurden mit dem Ziel, möglichst parallele Grundflächen zu erhalten, mit Siliziumcarbid-Sandpapier bis zu einer Körnung von $6 \mu \mathrm{m}$ plan geschliffen. Die Wahl der Probengeometrie verhindert im PAC-Experiment selbst dann ein Austreten radioaktiver Sonden aus der Stempelanordnung, wenn die Probe unter Kompression versagt.

\subsubsection{Dünne Schichten}

Die $\mathrm{Cr}_{2}$ AlC-Dünnschichtproben wurden von V. Vishnyakov in bereits kristalliner Form auf einem Siliziumnitrid/Siliziumsubstrat zur Verfügung gestellt. Die Deposition der Schicht erfolgte mittels Ionenstrahl-Sputter-Deposition direkt auf die $200 \mathrm{~nm}$ dicke, amorphe Siliziumnitridpufferschicht, wobei das Sputtertarget, bestehend aus aus elementarem Chrom, Aluminium und Kohlenstoff, mit Argon-Ionen beschossen wurde. Nach der Deposition wurde die zunächst noch amorphe $\mathrm{Cr}_{2} \mathrm{AlC}$-Schicht für 30 Minuten bei $973 \mathrm{~K}$ ausgeheilt, um die kristalline MAX-Phase zu erhalten. Die finale Schicht ist 1,3(1) $\mu \mathrm{m}$ dick und enthält Kristallite mit einer Korngröße von einigen hundert Nanometern (siehe TEM-Analyse in Kapitel 5). Die Synthese einer vergleichbaren Probe ist in Referenz [16] beschrieben.

Analog zu den Volumenproben wurde die Schicht samt Substrat mit einer Wafersäge in $5 \times 6 \mathrm{~mm}^{2}$ große Stücke zerteilt. 


\section{Kapitel 4}

\section{Theoretische Ergebnisse}

Auf die Einführung des Konzeptes des Gradient-elastischen Tensors von Shulman [48] folgten eine Reihe von Experimenten, in denen es gelang, intrinsische Dehnungen in kubischen Materialien quantitativ zu beschreiben [91-96]. Der Erfolg dieser Messungen beruhte unter anderem auf der Möglichkeit, die entsprechenden Gradient-elastischen Tensoren an Einkristallen experimentell bestimmen zu können. Ist eine experimentelle Bestimmung nicht möglich, weil es sich z.B. um ein Kristallsystem mit geringer Symmetrie handelt oder um ein Material, das nur in polykristalliner Form vorliegt, muss der Tensor berechnet werden.

Elektrische Feldgradienten können in ionischen Kristallen oft schon durch ein Punktladungsmodell hinreichend gut beschrieben werden, die meisten anderen Fälle bedürfen aber einer vollständigen Beschreibung der elektronischen Zustandsdichte. Gleiches gilt für die Bestimmung des Gradient-elastischen Tensors, der den elektrischen Feldgradienten am Kern eines Sondenatoms mit der Dehnung koppelt. Dieser Ansatz wird in diesem Kapitel verfolgt. $^{a}$

Ausgehend von der Symmetrie um die Sonde und den Berechnungen der elektronischen Zustandsdichte werden die Komponenten des Gradient-elastischen Tensors für zehn Modellsysteme und vier MAX-Phasen bestimmt. Die so ermittelten Konstanten werden später mit experimentellen Werten verglichen, um die Genauigkeit der Methode einschätzen zu können. Anschließend werden daraus EFG-Verteilungen in MAX-Phasen berechnet, wie sie unter dem Einfluss bestimmter Spannungsfelder entstehen. Die hier relevanten Spannungsfelder werden durch elastische Verzerrungen um Defekte oder durch Kompressionen des Materials hervorgerufen. Die dadurch erhaltenen Vorhersagen bilden die Diskussionsgrundlage der PAC-Experimente an deformierten MAX-Phasen (siehe Kapitel 5 und 6). An geeigneter Stelle, auch im auf dieses Kapitel folgenden experimentellen Teil, wird auf andere Systeme zurückgegriffen, um die Grenzen der Methode aufzuzeigen.

\subsection{Bestimmung Gradient-elastischer Tensoren ${ }^{b}$}

Die neben den vier MAX-Phasen untersuchten Materialsysteme umfassen zehn Systeme, deren Gradient-elastische Konstanten oder davon abgeleitete Größen experimentell bestimmt wurden. Ein direkter Vergleich ist für die regulären Gitterplätze in den kubisch-

\footnotetext{
${ }^{a}$ Die dafür nötigen Dichtefunktionalrechnungen wurden auf den Parallelrechnern der Gesellschaft für wissenschaftliche Datenverarbeitung Göttingen (GWDG) durchgeführt.

${ }^{b}$ Dieses Kapitel basiert im Wesentlichen auf Ergebnissen, die in Referenz [185] veröffentlicht wurden.
} 


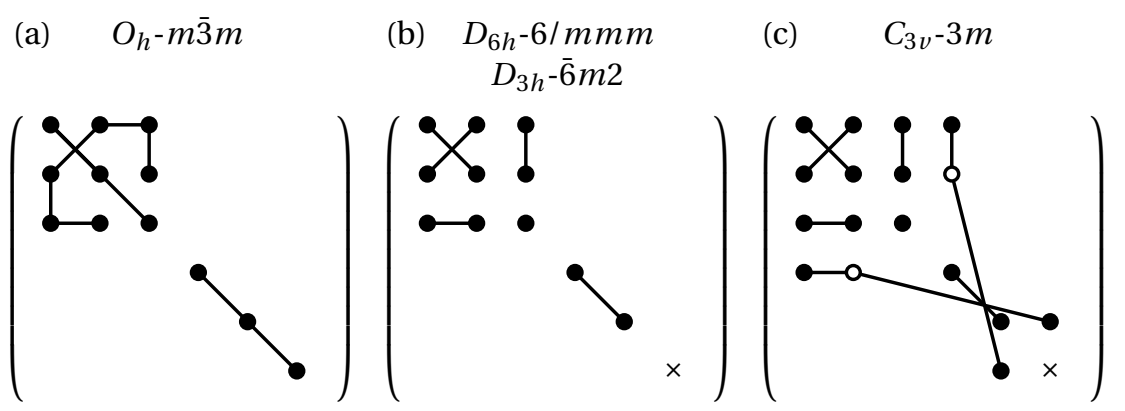

Abbildung 4.1: Struktur der Gradient-elastischen Tensoren $S_{m n}$ und $C_{m n}$ in Voigt'scher Notation für ausgewählte Punktgruppen: (a) $O_{h}-m \overline{3} m$ (regulärer Gitterplatz in fcc- und bccMetallen), (b) $D_{6 h^{-}}-6 / \mathrm{mmm}$ (regulärer Gitterplatz in hcp-Metallen) sowie $D_{3 h^{-}-\overline{6} m 2}$ (Substitutionsatom in hcp-Metallen und MAX-Phasen) und (c) $C_{3 v}-3 m$ (Substitutionsatom in $\mathrm{ZnO}$ ). Die Strukturbestimmung folgt aus den von Fumi beschriebenen Methoden [74, 75]. Nicht-verschwindenden Komponenten sind durch einen schwarzen Punkt $(\bullet)$ symbolisiert und mit gleichen Elementen direkt verknüpft. Kreise (o) sind von gleichem Betrag wie ihre verknüpften Elemente, unterscheiden sich aber in ihrem Vorzeichen. $\times$ entspricht $1 / 2\left(S_{11}-S_{12}\right)$ oder $1 / 2\left(C_{11}-C_{12}\right)$. Die Anzahl der unabhängigen Komponenten lässt sich aufgrund der Spurfreiheit des EFGTensors noch weiter auf zwei (a), vier (b) bzw. sechs (c) reduzieren.

flächenzentrierten Metallen Aluminium [81, 82], Kupfer [79] und Gold [87] sowie den kubisch-raumzentrierten Metallen Molybdän [85], Niob [84] und Tantal [83, 86] möglich. Weitere geeignete Vergleichssysteme bilden die hexagonalen Systeme Cadmium [89, 99, 100], Titan, Zink [124] und Zinkoxid [88], wobei zu Titan entsprechende Messungen in Kapitel 5 präsentiert werden. Anders als in den kubischen Metallen wird der Gradientelastische Tensor in den hexagonalen Systemen, also auch den MAX-Phasen, an einer in das Material substitutionell eingebrachten Cadmiumsonde ermittelt. Die Sonde befindet sich in Zinkoxid auf dem Zink-Platz und in den MAX-Phasen auf dem A-Platz.

\subsubsection{Vereinfachungen durch die Punktgruppe der Sonde}

Der Gradient-elastische Tensor enthält aufgrund der Symmetrie und der Spurfreihet des EFG-Tensors sowie der Symmetrie des Spannungs- bzw. Dehnungstensors maximal 30 unabhängige Komponenten (siehe Kapitel 2.2). Diese Anzahl kann noch weiter reduziert werden, wenn die Punktgruppe des Sondenatoms analysiert wird.

Die Punktgruppe ist in allen kubischen Metallen $O_{h}$, womit direkt aus Referenz [74] und unter Berücksichtigung der Spurfreiheit des EFGs (siehe Gleichung 2.6) folgt, dass der entsprechende Gradient-elastische Tensor lediglich aus zwei unabhängigen Komponenten besteht [48]. Äquivalente und verschwindende Komponenten dieses Tensors sind in Abbildung 4.1a in Voigt'scher Notation dargestellt. Die Punktgruppe $O_{h}$ fordert, dass der Gradient-elastische Tensor symmetrisch ist.

Die Auswertung der Symmetrien in den hexagonalen Systemen erfolgt mit Referenz [75]. Für die Punktgruppen $D_{6 h}$ und $D_{3 h}$, die der Punktgruppe regulärer Gitterplätze in Cadmi- 
um bzw. einer Cadmiumsonde in hexagonalen Metallen und MAX-Phasen entsprechen, ergibt sich die in Abbildung 4.1b dargestellte Struktur des Gradient-elastischen Tensors. Zusammen mit der Spurfreiheit des EFGs folgt, dass in diesen Fällen vier unabhängige Komponenten den unsymmetrischen Tensor bestimmen. Zur Beschreibung des Gradientelastischen Tensors an der Cadmiumsonde in Zinkoxid werden sechs unabhängige Komponenten benötigt, die entsprechende Punktgruppe lautet $C_{3 \nu}$. Anhand der in Abbildung 4.1c gezeigten Struktur wird deutlich, dass eine Dehnung entlang der Basalebenen Scherkomponenten des EFGs hervorrufen. Dies ist in keinem anderen hier untersuchten System der Fall. Es kann somit erwartet werden, dass sich Dehnungen in der Basalebene von Zinkoxid anisotrop auf den EFG auswirken.

\subsubsection{Ab-initio Berechnung der unabhängigen Konstanten}

Die Bestimmung der unabhängigen Gradient-elastischen Konstanten $S_{m n}$ erfolgt mit dem Programm WIEN2k [118] im Rahmen der Dichtefunktionaltheorie (siehe Kapitel 2.3) durch dehnungsabhängige Berechnungen des Feldgradienten. Ausgehend von Rechnungen im nicht gedehnten Gitter werden im Folgenden die Auswirkungen der Dehnungen auf den EFG untersucht. Phononenbeiträge zum EFG und somit Temperatureffekte [72] werden im Folgenden nicht berücksichtigt. Alle berechneten Gradient-elastischen Konstanten sind daher nur bei $T=0 \mathrm{~K}$ gültig.

$\mathrm{Zu}$ Beginn einer Rechnung werden die Atompositionen des untersuchten Materials benötigt, die durch Gittervektoren $\vec{r}_{i}$ aufgespannt werden. Diese sind in der Regel durch die Raumgruppe des Kristalls vorgegeben, lediglich die Gitterkonstanten und freie interne Parameter müssen gewählt werden. Um sicherzustellen, dass alle Rechnungen im Grundzustand durchgeführt werden, ist es notwendig, die Gitterkonstanten zu optimieren. Im Zuge dessen wird die Gesamtenergie des Systems als Funktion des Volumens und, im Falle der hexagonalen Systeme, des $c / a$-Verhältnisses simultan mit etwaigen internen Parametern minimiert. Das Minimum wird über eine Anpassung der Gesamtenergie mit einer quadratischen Gleichung in einer bzw. zwei Variablen erreicht. Die so ermittelten Gitterkonstanten (siehe Anhang A) dienen als Ausgangspunkt für die Berechnung der Gradientelastischen Konstanten.

Die verwendeten Eingangsparameter für die MAX-Phasen [47], Zink [120] und Zinkoxid [121] entsprechen denen vorheriger Arbeiten, für alle anderen Systeme sind die entsprechenden Parameter in Anhang A aufgeführt. In Systemen mit eingebrachtem Sondenatom wird - bevor das entsprechende Atom eingesetzt wird - eine möglichst große, ungefähr kubische Superzelle aus der Einheitszelle konstruiert, um den Einfluss der Störstelle auf das umgebende Gitter näherungsweise berücksichtigen zu können. Die maximale Größe dieser Superzelle ist durch die Rechenzeit begrenzt. Durch das eingebrachte Sondenatom entsteht ein Symmetriebruch, der eine Relaxation der umgebenden Atome ermöglicht. Die internen Positionen werden mit der PORT-Routine [118] variiert, bis die internen Kräfte einen Schwellwert $F_{\text {tol }}$ (siehe Anhang A) unterschreiten. Die abschließende selbstkonsistente Berechnung der Ladungsdichte erfolgt bis zu einer Ladungskonvergenz von $10^{-6} e$. Die gewählten Eingangsparameter werden nach Möglichkeit im späteren Verlauf beibehalten.

Die sich durch die Rechnung im Grundzustand ergebenen elektrischen Feldgradienten 
sind, so sie nicht verschwinden, alle axialsymmetrisch. In Tabelle A.2 sind die berechneten Feldgradient $V_{z Z}^{\text {calc }}$ den experimentellen Werten $V_{z z}^{\exp }[47,186-189]$ unter Ausnutzung eines Quadrupolmomentes von $Q=0,765$ (15) b [120] gegenüber gestellt. Die entsprechenden Tieftemperaturmessungen in $\mathrm{Ti}_{2} \mathrm{AlN}$ werden mangels Literaturwerten in Kapitel 5.3 gezeigt.

Ausgehend von den Rechnungen im Grundzustand werden nun Verzerrungen der durch die Gittervektoren $\vec{r}_{i}$ aufgespannten Zelle vorgenommen. Die Dehnung wird, analog zur ab-initio Berechnung elastischer Konstanten [190], über eine Verzerrung der Gittervektoren $\vec{r}_{i}^{\prime}=D \cdot \vec{r}_{i}$ mit der Verzerrungsmatrix

$$
D=\left(\begin{array}{ccc}
1+\varepsilon_{x x} & \gamma_{x y} / 2 & \gamma_{x z} / 2 \\
\gamma_{x y} / 2 & 1+\varepsilon_{y y} & \gamma_{y z} / 2 \\
\gamma_{x z} / 2 & \gamma_{y z} / 2 & 1+\varepsilon_{z z}
\end{array}\right)
$$

eingebracht. Die neuen Gitterkonstanten $a^{\prime}, b^{\prime}, c^{\prime}, \alpha^{\prime}, \beta^{\prime}$ und $\gamma^{\prime}$ ergeben sich aus Norm und Skalarprodukt der Gittervektoren $\vec{r}_{i}{ }^{\prime}$. Eine Untersuchung der verzerrten Zelle mit der SGROUP-Routine [118] ermöglicht es, alle noch auftretenden Symmetrien zu bestimmen und für die Dichtefunktionalrechnung nutzbar zu machen. Dies erhöht zum einen die Genauigkeit der Rechnungen, zum anderen minimiert es den Rechenaufwand. Der aus dem verzerrten Gitter berechnete Tensor des elektrischen Feldgradienten ist vollständig, das heißt alle Komponenten sind auf einmal zugänglich. Dies hat zur Folge, dass unabhängig von der Kristallklasse maximal sechs voneinander unabhängige Dehnungen nötig sind, um alle Komponenten des Gradient-elastischen Tensors zu ermitteln.

Für die kubischen Systeme werden zwei unabhängige Dehnungen, $\varepsilon_{z z}$ und $\gamma_{y z}$, verwendet. Die erste Dehnung führt zu einem tetragonalen, die zweite zu einem orthorhombischen Kristallsystem. Im Falle der Scherdehnung muss beachtet werden, dass sich das neue Koordinatensystem gegenüber dem alten um $\theta= \pm \pi / 4$ um die x-Achse dreht. Die Richtung der Drehung hängt hierbei davon ab, ob es sich um einen kubisch-flächen- oder kubischraumzentrierten Kristall handelt. Entsprechend müssen die erhaltenen EFG-Tensoren mit Hilfe der Drehmatrix $R_{x}(\theta)$ gemäß $R_{x}(\theta)^{T} \cdot V_{i j} \cdot R_{x}(\theta)$ transformiert werden.

Für die hexagonalen Systeme werden drei unabhängige Dehnungen verwendet: eine Dehnung $\varepsilon_{z z}{ }^{a}$, die die Symmetrie erhält, und die Dehnungen $\varepsilon_{y y}$ sowie $\gamma_{y z}$, die beide die Symmetrie brechen. Im Falle der Punktgruppen $D_{6 h}$ und $D_{3 h}$ führt die Dehnung $\varepsilon_{y y} \mathrm{zu}$ einer orthorhombischen, die Dehnung $\gamma_{y z}$ zu einer monoklinen Symmetrie. Das Koordinatensystem ist nach der Scherdehnung des Systems mit Punktgruppe $D_{3 h}$ um

$$
\theta=\arccos \left(\left(c_{0}-a_{0} \cdot \gamma_{y z}\right) / \sqrt{a_{0}^{2}+c_{0}^{2}-2 a_{0} c_{0} \gamma_{y z}}\right)
$$

um die x-Achse gedreht. Für das System der Punktgruppe $C_{3 v}$ ergibt sich bei $\varepsilon_{y y}$ eine monokline und bei $\gamma_{y z}$ eine trikline Symmetrie. Das Koordinatensystem ist in monokliner Darstellung gegenüber dem alten um $\theta=\arctan \left(\sqrt{3} a_{0} / c_{0}\right)$ um die y-Achse gedreht.

Noch bevor die eigentlichen Berechnungen durchgeführt werden, können allgemeine Kriterien zur Überprüfung und Sicherstellung ihrer Konsistenz formuliert werden. In allen Rechnungen, bei denen ein Symmetriebruch auftritt, muss sichergestellt werden, dass

${ }^{a}$ In allen hexagonalen Systemen wird die c-Achse des Kristalls implizit als z-Achse angenommen. 
sich für kleine Dehnungen näherungsweise der ursprüngliche EFG $V_{i j}^{0}$ ergibt. Des Weiteren sollten sich die Komponenten des EFG-Tensors entsprechend der durch die Punktgruppe vorgegebenen Struktur des Gradient-elastischen Tensors verändern. Dieses Kriterium wird meist schon durch die vorgegebenen Symmetrien des verzerrten Gitters implizit erfüllt. Dies gilt insbesondere dann, wenn einzelne Komponenten des EFG-Tensors aus Symmetriegründen ohnehin verschwinden. Darüber hinaus wird eine lineare Änderung der Komponenten des EFGs mit der Dehnung erwartet. Trifft dies nicht zu, ist davon auszugehen, dass in dem untersuchten Bereich die linear-elastische Theorie nicht anwendbar ist. Zuletzt muss sichergestellt werden, dass in Systemen mit internen Freiheitsgraden, wie zum Beispiel der Atomanordnung um ein eingebrachtes Sondenatom, eine Optimierung der Positionen bei jeder Dehnung stattfindet. Diese Kriterien treffen, falls nicht explizit anders erwähnt, auf alle folgenden Rechnungen zu.

Die berechneten Komponenten des EFG-Tensors der kubischen Systeme sind als Funktion der jeweiligen Dehnung in Abbildungen 4.2 dargestellt. Alle Feldgradienten ändern sich linear mit der Dehnung, wobei die Steigung die gesuchten Konstanten $S_{m n}$ ergibt. Werte und Fehler von $S_{m n}$ werden aus den Parametern eines linearen Fits bestimmt. Der Fehler spiegelt somit die Streuung der Daten wieder und bietet dadurch ein Maß, die Qualität der Rechnung einzuschätzen. Der bei kleinsten Dehnungen durch den Symmetriebruch fälschlich erzeugte EFG ist in der Regel deutlich kleiner als die Dynamik des EFGs. Lediglich in der tetragonalen Verzerrung von Kupfer zeigt sich aufgrund der geringen Dynamik eine relativ starke Asymmetrie um null. Der Einfluss dieser Asymmetrie auf die Bestimmung der Gradient-elastischen Konstanten kann vernachlässigt werden, da hierfür nur die Steigung relevant ist. Die restlichen Komponenten, die nach der Struktur des Gradientelastischen Tensors eigentlich konstant bleiben sollten, zeigen ein parabolisches Verhalten mit einer Dynamik von bis zu 1\% der sich linear ändernden Komponenten. Diese Beiträge werden im Rahmen der hier verwendeten linear-elastischen Theorie vernachlässigt. Die Vorzeichen der Konstanten sind in allen untersuchten kubischen Systemen gleich, der Betrag hingegen variiert über zwei Größenordnungen.

Die berechneten Komponenten des EFG-Tensors der verzerrten hexagonalen Metalle sind in Abbildung 4.3 gezeigt. Für Cadmium ergibt sich ein ähnliches Bild wie in den kubischen Metallen, die Streuung ist gering und die Änderung linear. Lediglich die Dynamik der restlichen, sich parabolisch ändernden Komponenten ist mit $6 \%$ relativ stark, jedoch noch immer vernachlässigbar.

Beim Übergang hin zu Systemen, in denen die betrachtete Sonde zunächst in eine Superzelle eingebracht werden muss, ändert sich die Genauigkeit der ermittelten Konstanten erheblich. So zeigt sich für Zink noch klar ersichtlich ein lineares Verhalten, wenngleich die Streuung der Daten um die Ausgleichsgerade stark zunimmt. Die stärkere Streuung der Daten hängt im Wesentlichen mit der Optimierung der internen Positionen und dem dabei verwendeten Toleranzkriterium $F_{\text {tol }}$ zusammen. Für $F_{\text {tol }}$ muss ein Kompromiss zwischen akzeptabler Streuung und Rechenzeit gefunden werden. Die Optimierung hingegen wegzulassen ist, wie später bei Zinkoxid exemplarisch demonstriert wird, keine Option. Die Streuung zeigt sich auch in denen sich sonst parabolisch verhaltenden, restlichen EFGKomponenten, mit der Folge, dass das parabolische Verhalten in Systemen mit fremden Sondenatom nicht mehr sichtbar ist. Dies trifft auch auf alle folgenden Systeme zu. 

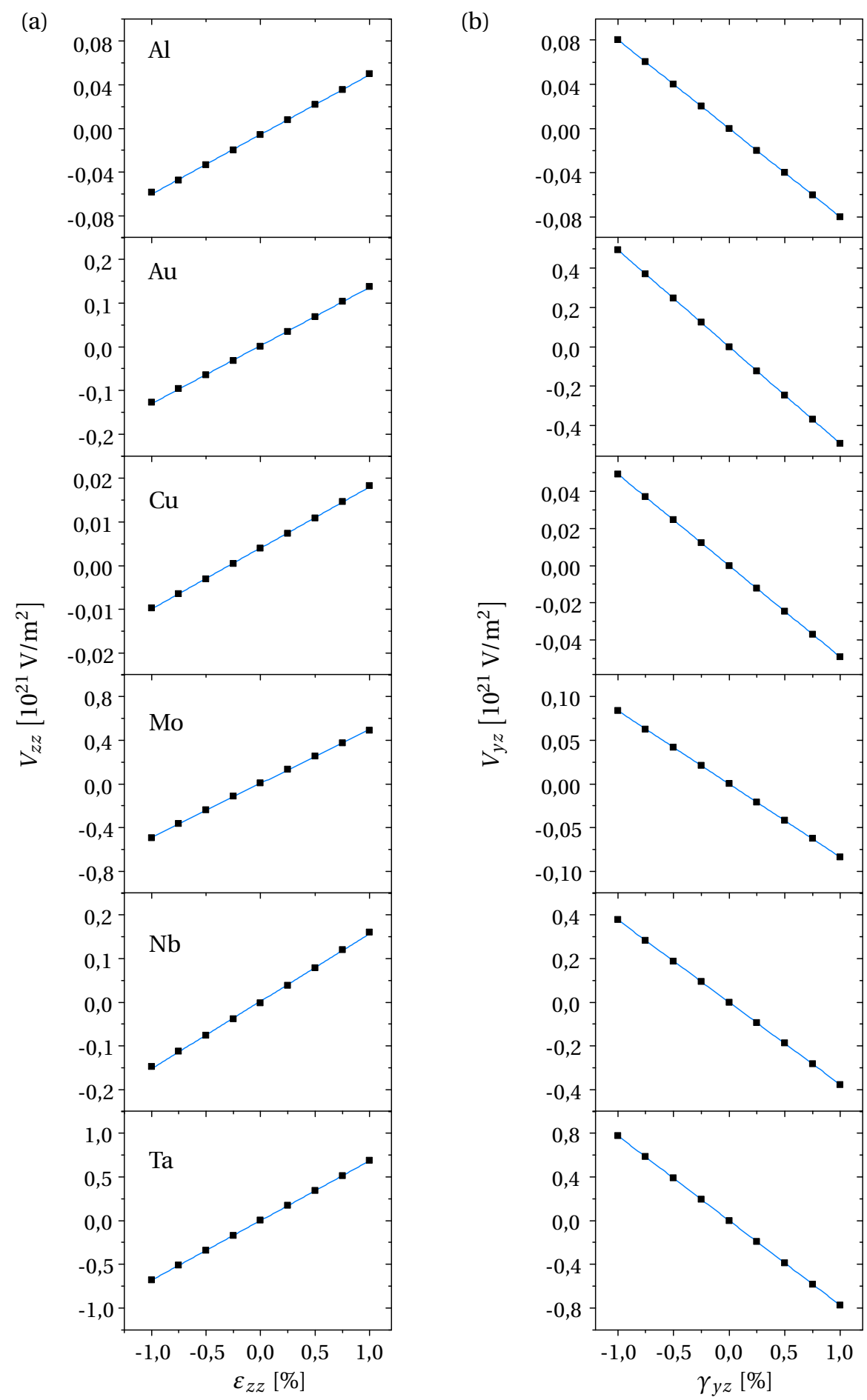

Abbildung 4.2: Berechnete EFG-Tensorkomponenten $V_{i j}$ an regulären Gitterplätzen kubischer Metalle als Funktion einer Dehnung $\varepsilon_{z z}$ (a) sowie einer Scherdehnung $\gamma_{y z}$ (b). Alle relevanten Tensorkomponenten verändern sich linear mit der Dehnung. Die eingezeichneten Linien entsprechen einem linearen Fit der Daten (siehe Tabelle 4.1). 

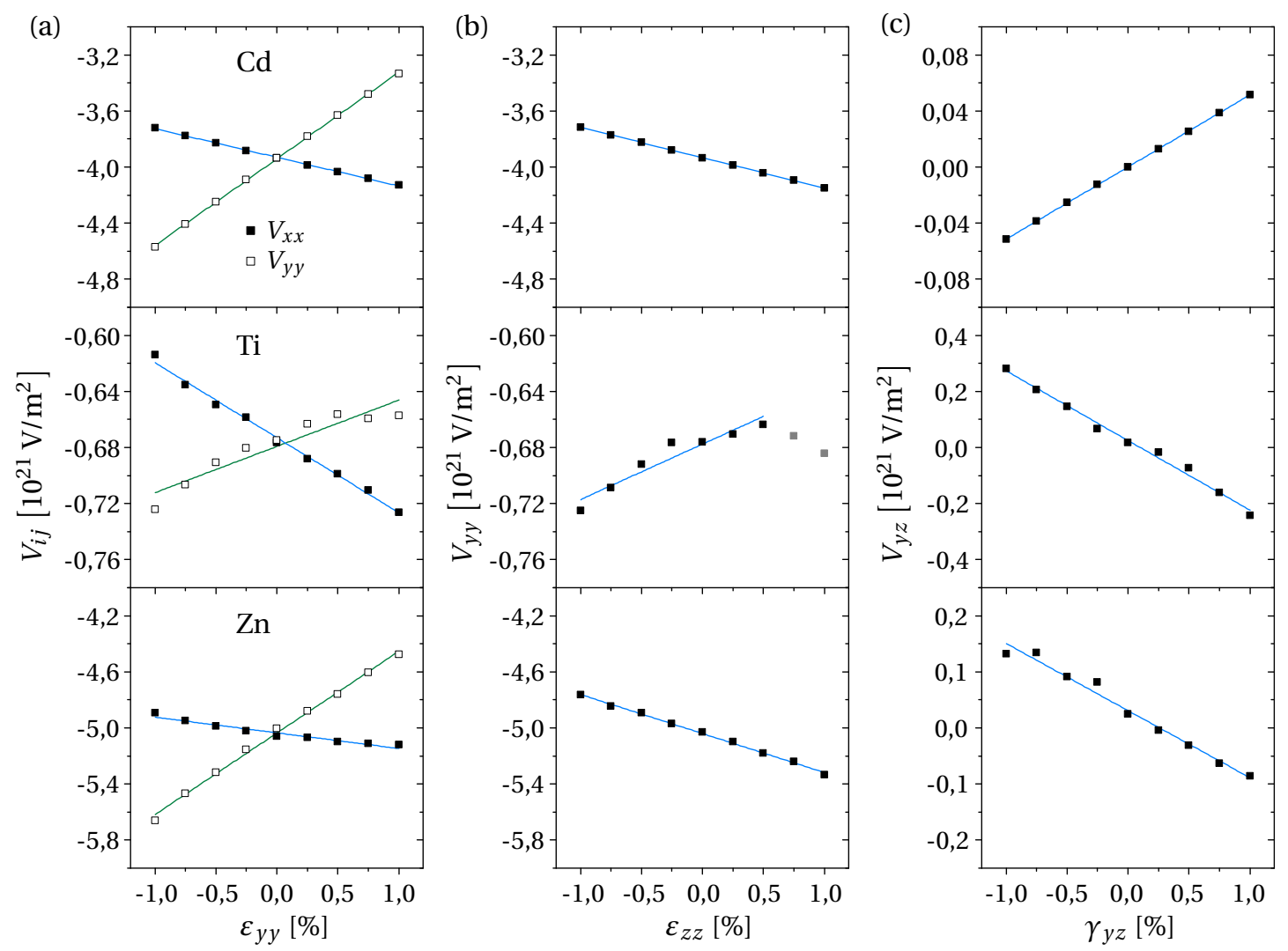

Abbildung 4.3: Berechnete Komponenten $V_{i j}$ des EFG-Tensors an der Cadmiumsonde in hexagonalen Metallen als Funktion einer Dehnung $\varepsilon_{y y}$ (a), einer Dehnung $\varepsilon_{z z}$ (b) und einer Scherdehnung $\gamma_{y z}$ (c). Die relevanten Tensorkomponenten in Cadmium und Zink verhalten sich, anders als in Titan, linear mit der Dehnung. Die eingezeichneten Linien entsprechen einem linearen Fit der schwarzen Datenpunkte (siehe Tabelle 4.2). Graue Punkte sind vom Fit ausgenommen.

An der Cadmiumsonde in Titan werden erste Grenzen der linear-elastischen Theorie sichtbar. Schon für kleine Dehnungen $\varepsilon_{z z}$ zeigen sich deutliche Abweichungen vom linearen Verhalten. Um dennoch einen Anhaltspunkt für die entsprechende Gradient-elatische Konstante zu erhalten, wird die lineare Anpassung auf Dehnungen zwischen - $1 \%$ und 0,5\% beschränkt. Der Fehler der daraus abgeleiteten Konstante wird mit 30\% abgeschätzt. Dieses Verhalten zeigt sich in Ansätzen auch bei einer Dehnung $\varepsilon_{y y}$, wird hier aber durch den Fehler der Streuung kaschiert. Der Ursprung dieses Verhaltens ist unklar, allerdings zeigt eine vergleichbare Rechnung in reinem Titan keine Abweichung vom linearen Verhalten. Es kann also davon ausgegangen werden, dass diese Abweichung erst durch die Cadmiumsonde verursacht wird.

Die Dehnungsabhängigkeit des EFGs an der Cadmiumsonde in den MAX-Phasen ist in Abbildung $4.4 \mathrm{zu}$ sehen. Das gewählte Toleranzkriterium $F_{\text {tol }}$ ist für die meisten Rechnungen ausreichend, wie an der geringen Streuung deutlich wird. Eine Besonderheit tritt in $\mathrm{Ti}_{2} \mathrm{AlC}$ auf. Anders als in allen anderen untersuchten Systemen ergibt sich hier mit den 

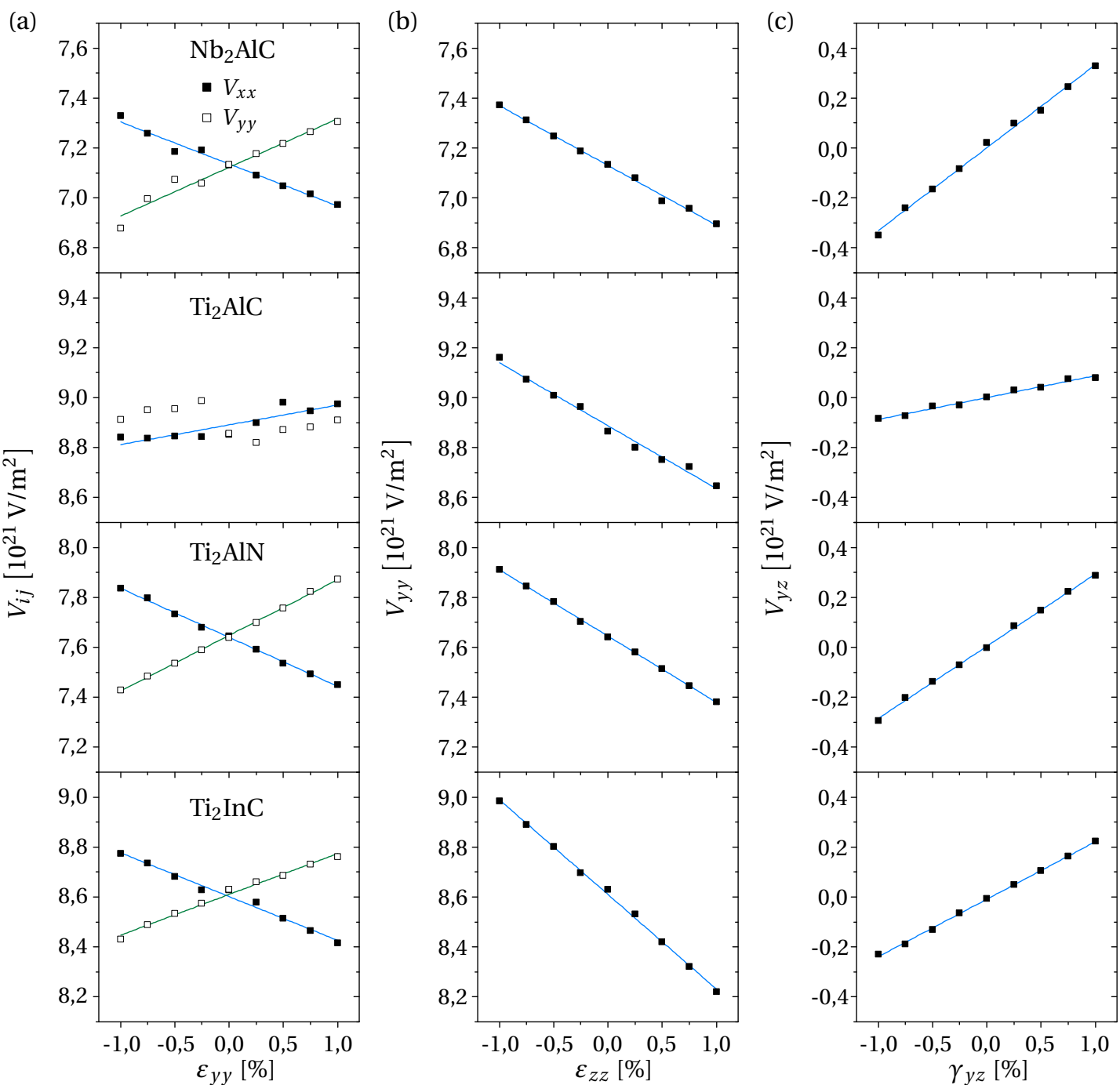

Abbildung 4.4: Berechnete Komponenten $V_{i j}$ des EFG-Tensors an der Cadmiumsonde in MAXPhasen als Funktion einer Dehnung $\varepsilon_{y y}$ (a), einer Dehnung $\varepsilon_{z z}$ (b) und einer Scherdehnung $\gamma_{y z}$ (c). Fast alle relevanten Tensorkomponenten ändern sich linear mit der Dehnung. Die Steigung ist zwischen den MAX-Phasen vergleichbar, einzig $\mathrm{Ti}_{2} \mathrm{AlC}$ weicht von den anderen MAX-Phasen deutlich ab; die Abhängigkeit der Komponente $V_{y y}$ von der Dehnung $\varepsilon_{y y}$ wird lediglich abgeschätzt. Die eingezeichneten Linien entsprechen einem linearen Fit der Daten (siehe Tabelle 4.2). 
(a)

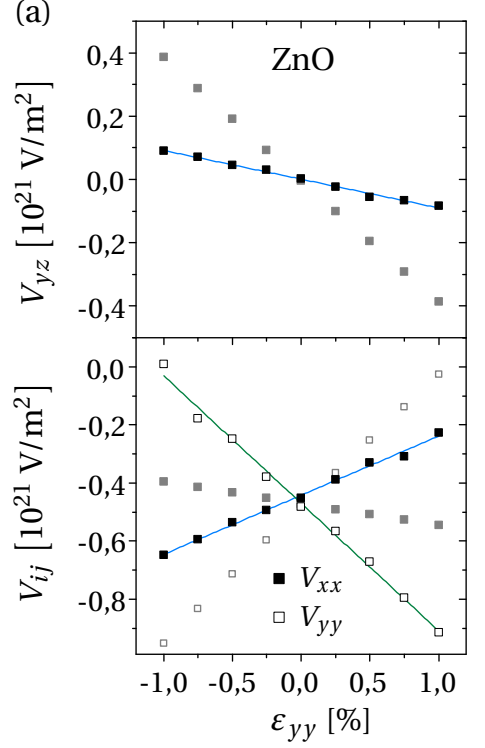

(b)

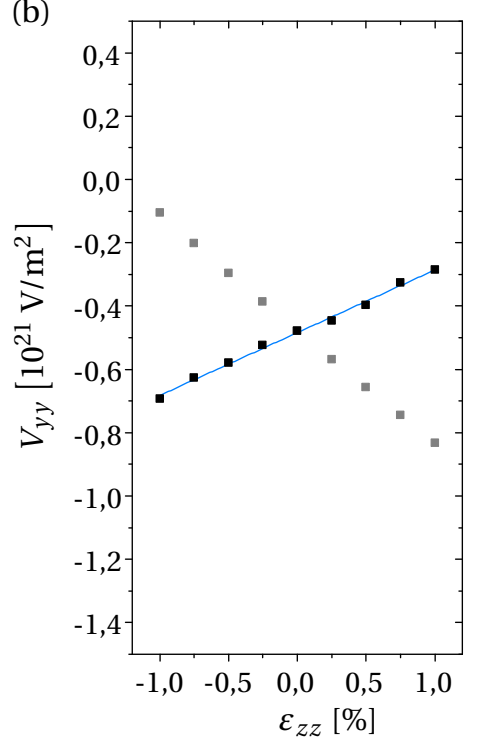

(c)

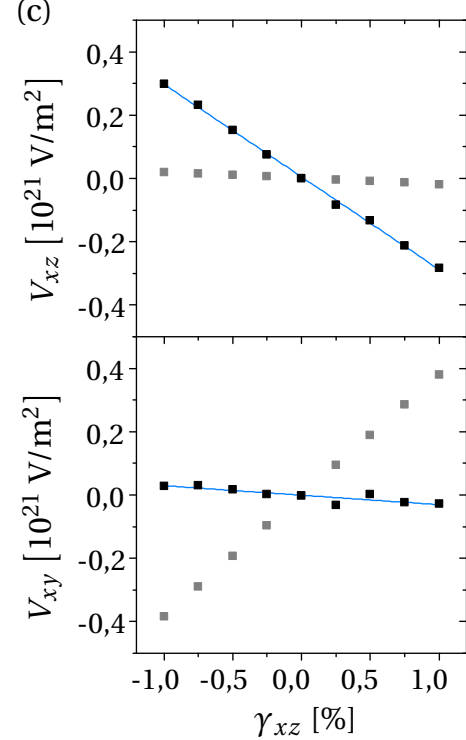

Abbildung 4.5: Berechnete Komponenten $V_{i j}$ des EFG-Tensors an der Cadmiumsonde in Zinkoxid als Funktion einer Dehnung $\varepsilon_{y y}$ (a), einer Dehnung $\varepsilon_{z z}$ (b) und einer Scherdehnung $\gamma_{y z}$ (c). Alle relevanten Tensorkomponenten ändern sich linear mit der Dehnung. Die eingezeichneten Linien entsprechen einem linearen Fit der Daten (siehe Tabelle 4.2). Die Feldgradienten vor der Optimierung der internen Positionen sind zum Vergleich in grau dargestellt.

sonst üblichen Eingangsparametern in orthorhombischer Aufstellung bei verschwindender Dehnung eine starke Abweichung vom axialsymmetrischen EFG. Diese Asymmetrie kann durch eine höhere Dichte der k-Punkte in der ersten Brillouinzone wieder entfernt werden. Es ist nicht klar, warum dieses Artefakt lediglich in $\mathrm{Ti}_{2} \mathrm{AlC}$ auftritt, da das k-Gitter in allen MAX-Phasen identisch ist. Dieser Korrektur zum Trotz, zeigt der EFG an der Cadmiumsonde in $\mathrm{Ti}_{2} \mathrm{AlC}$ ein von den anderen MAX-Phasen verschiedenes Verhalten. So ändert sich der EFG unter den Dehnungen $\varepsilon_{y y}$ und $\gamma_{y z}$ nur relativ schwach.

In Abbildung 4.5 sind die dehnungsabhängigen Änderungen des EFGs an der Cadmiumsonde in Zinkoxid dargestellt. Gemäß der Struktur des Gradient-elastischen Tensors sind hier sechs Konstanten zu bestimmen. Die Streuung der Datenpunkte um den linearen Zusammenhang ist vergleichsweise klein. Zum Vergleich sind die Feldgradienten mit eingezeichnet, wie sie vor der Optimierung der internen Parameter erhalten werden. Hierbei wird die Relevanz der Optimierung besonders deutlich, da nicht einmal das Vorzeichen der Konstanten erhalten bleibt.

Eine Übersicht über die so ermittelten Gradient-elastischen Konstanten $S_{m n}$ und der davon abgeleiteten Konstanten $C_{m n}=\sum_{k=1}^{6} S_{m k} s_{k n}$ ist für die kubischen Systeme in Tabelle 4.1, für die hexagonalen Systeme in Tabelle 4.2 gegeben. Hierbei kommen nach Möglichkeit die experimentellen elastischen Konstanten des jeweiligen Volumenmaterials bei $T=0 \mathrm{~K}$ [55-57, 59-63] zum Einsatz. Ist dies nicht möglich, werden entweder berechnete Konstanten[40] oder experimentelle Konstanten bei Raumtemperatur [58] verwendet (siehe Tabelle 2.1). 
Tabelle 4.1: Berechnete Gradient-elastische Konstanten $S_{m n}$ und $C_{m n}$ an regulären Gitterplätzen kubischer Metalle mit Punktgruppe $O_{h}$.

\begin{tabular}{|c|c|c|c|c|c|c|c|}
\hline \multicolumn{2}{|c|}{ Wirtsgitter } & $\mathrm{Al}$ & $\mathrm{Au}$ & $\mathrm{Cu}$ & Mo & $\mathrm{Nb}$ & $\mathrm{Ta}$ \\
\hline$S_{11}$ & $10^{21} \mathrm{~V} / \mathrm{m}^{2}$ & $5,46(5)$ & $13,28(9)$ & $1,39(1)$ & $49,2(3)$ & $15,4(2)$ & $68,2(1)$ \\
\hline$S_{44}$ & $\left.10^{21} \mathrm{~V} / \mathrm{m}^{2}\right]$ & $-8,02(1)$ & $-49,42(1)$ & $-4,931(1)$ & $-8,37(1)$ & $-37,66(5)$ & $-77,5(1)$ \\
\hline$C_{11}$ & $\left./ \mathrm{m}^{2} \cdot \mathrm{GPa}^{-1}\right]$ & $0,105(1)$ & $0,415(3)$ & $0,0272(2)$ & $0,178(2)$ & $0,138(2)$ & 0,631(1) \\
\hline$C_{44}$ & $\left./ \mathrm{m}^{2} \cdot \mathrm{GPa}^{-1}\right]$ & $-0,259(1)$ & $-1,098(1)$ & $-0,0601(1)$ & $-0,0670(1)$ & $-1,299(2)$ & $-0,891(1)$ \\
\hline
\end{tabular}

Tabelle 4.2: Berechnete Gradient-elastische Konstanten $S_{m n}$ und $C_{m n}$ an der Cadmiumsonde in hexagonalen Metallen, MAX-Phasen und Zinkoxid. Zum Vergleich sind die berechneten und die gemessenen Feldgradienten vor der Dehnung mit angegeben.

\begin{tabular}{|c|c|c|c|c|c|c|c|c|c|c|}
\hline \multicolumn{3}{|c|}{$\begin{array}{c}\text { Wirtsgitter } \\
\text { Sondenatom } \\
\text { Punktgruppe }\end{array}$} & $\begin{array}{c}\mathrm{Cd} \\
\mathrm{Cd} \\
D_{6 h}\end{array}$ & $\begin{array}{c}\mathrm{Ti} \\
\mathrm{Cd} \\
D_{3 h}\end{array}$ & $\begin{array}{c}\mathrm{Zn} \\
\mathrm{Cd} \\
D_{3 h}\end{array}$ & $\begin{array}{c}\mathrm{Nb}_{2} \mathrm{AlC} \\
\mathrm{Cd} \\
D_{3 h}\end{array}$ & $\begin{array}{c}\mathrm{Ti}_{2} \mathrm{AlC} \\
\mathrm{Cd} \\
D_{3 h}\end{array}$ & $\begin{array}{c}\mathrm{Ti}_{2} \mathrm{AlN} \\
\mathrm{Cd} \\
D_{3 h}\end{array}$ & $\begin{array}{c}\mathrm{Ti}_{2} \operatorname{InC} \\
\mathrm{Cd} \\
D_{3 h}\end{array}$ & $\begin{array}{l}\mathrm{ZnO} \\
\mathrm{Cd} \\
C_{3 v}\end{array}$ \\
\hline$V_{z z}^{\text {calc }}$ & & {$\left[10^{21} \mathrm{~V} / \mathrm{m}^{2}\right]$} & 7,870 & 1,352 & 10,103 & $-14,260$ & $-17,730$ & $-15,283$ & $-17,258$ & 0,957 \\
\hline $\mid V_{z z}^{\text {exp }}$ & $\mathrm{p}_{(0 \mathrm{~K})}$ & {$\left[10^{21} \mathrm{~V} / \mathrm{m}^{2}\right]$} & $7,5(2)[186]$ & $1,79(2)[187]$ & $7,38(8)[188]$ & $13,4(3)[47]$ & $15,3(3)[47]$ & $14,1(3)^{a}$ & $16,2(3)[47]$ & $1,69(4)[189]$ \\
\hline$S_{11}$ & & {$\left[10^{21} \mathrm{~V} / \mathrm{m}^{2}\right]$} & $61,8(4)$ & $3,3(5)$ & $58(2)$ & $20(2)$ & $-4(3)$ & $22,3(3)$ & $16,3(7)$ & $-43,9(13)$ \\
\hline$S_{12}$ & & {$\left[10^{21} \mathrm{~V} / \mathrm{m}^{2}\right]$} & $-20,4(3)$ & $-5,3(2)$ & $-11(1)$ & $-17(1)$ & $8(2)$ & $-19,6(4)$ & $-17,5(8)$ & $20,4(6)$ \\
\hline$S_{13}$ & & {$\left[10^{21} \mathrm{~V} / \mathrm{m}^{2}\right]$} & $-21,7(1)$ & $4,0(15)^{b}$ & $-27,8(6)$ & $-24,0(6)$ & $-25(1)$ & $-26,5(3)$ & $-37,9(6)$ & $19,8(6)$ \\
\hline$S_{14}$ & & {$\left[10^{21} \mathrm{~V} / \mathrm{m}^{2}\right]$} & - & - & - & - & - & - & - & $-3,0(7)$ \\
\hline$S_{41}$ & & {$\left[10^{21} \mathrm{~V} / \mathrm{m}^{2}\right]$} & - & - & - & - & - & - & - & $9,1(3)$ \\
\hline$S_{44}$ & & {$\left[10^{21} \mathrm{~V} / \mathrm{m}^{2}\right]$} & $5,15(1)$ & $-24,8(9)$ & $-12,0(7)$ & $33,2(8)$ & $8,9(4)$ & $28,9(5)$ & $23,0(3)$ & $-29,3(4)$ \\
\hline$C_{11}$ & $10^{21} \mathrm{~V}$ & $\left.\mathrm{~m}^{2} \cdot \mathrm{GPa}^{-1}\right]$ & $0,778(5)$ & $0,037(5)$ & $0,60(2)$ & $0,119(6)$ & $-0,002(10)$ & $0,117(2)$ & $0,103(3)$ & $-0,46(1)$ \\
\hline$C_{12}[$ & $10^{21} \mathrm{~V}$ & {$\left[\mathrm{~m}^{2} \cdot \mathrm{GPa}^{-1}\right]$} & $-0,128(4)$ & $-0,060(4)$ & $0,11(1)$ & $-0,046(5)$ & $0,048(8)$ & $-0,056(2)$ & $-0,053(4)$ & $0,266(7)$ \\
\hline$C_{13}[$ & $10^{21} \mathrm{~V}$ & $\left.\mathrm{~m}^{2} \cdot \mathrm{GPa}^{-1}\right]$ & $-0,847(5)$ & $0,03(1)$ & $-0,97(2)$ & $-0,113(4)$ & $-0,104(5)$ & $-0,114(2)$ & $-0,169(3)$ & $0,189(6)$ \\
\hline$C_{14}[$ & $10^{21} \mathrm{~V}$ & {$\left[\mathrm{~m}^{2} \cdot \mathrm{GPa}^{-1}\right]$} & - & - & - & - & - & - & - & $-0,07(2)$ \\
\hline$C_{41}[$ & $10^{21} \mathrm{~V}$ & {$\left[\mathrm{~m}^{2} \cdot \mathrm{GPa}^{-1}\right]$} & - & - & - & - & - & - & - & $0,102(3)$ \\
\hline$C_{44}[$ & $10^{21} \mathrm{~V}$ & $\left.\mathrm{~m}^{2} \cdot \mathrm{GPa}^{-1}\right]$ & $0,215(1)$ & $-0,56(2)$ & $-0,21(2)$ & $0,239(6)$ & $0,082(4)$ & $0,231(4)$ & $0,267(4)$ & $-0,68(1)$ \\
\hline
\end{tabular}

${ }^{a}$ Diese Arbeit.

${ }^{b} \varepsilon_{z z} \in[-0,01 ; 0,05]$. 


\subsection{Verteilungen des elektrischen Feldgradienten}

Der Gradient-elastische Tensor erlaubt es, den lokalen elektrischen Feldgradienten unter Einfluss eines bestimmten Spannungs- oder Dehnungstensors darzustellen. Experimentelle Methoden, die den Feldgradienten messen, benötigen aber, egal ob es sich hierbei um PAC-, NMR- oder Mößbauerspektroskopie handelt, immer eine Vielzahl von Sondenatomen, messen also eine globale Verteilung von Feldgradienten. Diese globale Verteilung ist nur im Falle uniformer Spannungen unmittelbar durch Gleichung 2.5 bestimmt. In allen anderen Fällen muss sie aus den Eigenschaften des Spannungsfeldes hergeleitet werden. Die für diese Arbeit relevanten Spannungsfelder werden im Folgenden kontinuumstheoretisch am Beispiel von $\mathrm{Ti}_{2} \mathrm{AlN}$ analysiert.

Eine vergleichbare Analyse wurde bereits von Kanert [91] zur Beschreibung der Feldgradientenverteilung in defektreichen kubischen Systemen durchgeführt. Ausgehend vom verschwindenden EFG des kubischen Systems ist es möglich, näherungsweise eine analytische Beschreibung der Feldgradientenverteilung in der Umgebung von Punktdefekten und Versetzungen zu finden. Diese analytische Beschreibung ist im Falle von Systemen, in denen bereits ein intrinsischer EFG vorherrscht, nicht möglich, da hier dem Einfluss der Transformation ins Hauptachsensystem Rechnung getragen werden muss. Eine weitere Schwierigkeit liegt im Spannungsfeld der beteiligten Defekte, die in diesen Systemen, also auch den MAX-Phasen, im Allgemeinen komplexer sind, da anisotrope Eigenschaften des Gitters berücksichtigt werden müssen.

Beiden Umständen wird durch eine Monte-Carlo-Simulation begegnet, in der die anisotropen Spannungsfelder zufällig angeordneter Defekte überlagert und an einem Kernort ausgewertet werden. Durch eine Vielzahl unterschiedlicher Defektanordnungen wird die Verteilung der resultierenden Feldgradienten unter Berücksichtigung des individuellen Hauptachsensystems konstruiert. Die Eigenschaften der so erhaltenen Verteilungen sind charakteristisch für den jeweiligen Defekt und lassen sich im Idealfall in einen quantitativen Zusammenhang zur Defektdichte bringen. An geeigneter Stelle wird auf eine analytische Lösung zurückgegriffen, um beispielsweise die Proportionalitäten zwischen Verteilungsbreite und Defektdichte zu erläutern.

An die Frage der charakteristischen Feldgradientenverteilung eines Defektes schließt sich die Frage nach der elastischen Verbreiterung des Feldgradienten in polykristallinen Materialien an. Diese durch eine uniaxiale Spannung hervorgerufene Verbreiterung wurde bisher nicht untersucht; die zugehörige Verteilung wird im Folgenden auf analytische und numerische Weise beschrieben. Analog zur Defektdichte lassen sich hier die Eigenschaften der Verteilung mit der Spannung in einen quantitativen Zusammenhang bringen.

Bei allen Analysen dieser Art werden die Verteilung der größten EFG-Tensorkomponente $V_{z z}$ und des Asymmetrieparameters $\eta$ betrachtet. Eine mögliche Korrelation beider Parameter wird im Czjzek-Diagramm untersucht.

Die experimentell beobachtete Verteilung des Feldgradienten setzt sich aus der Faltung verschiedener Beiträge zusammen. Diese Faltung wird abschließend, insbesondere im Hinblick auf die in Kapitel 5 durchgeführten PAC-Messungen, diskutiert. Ungeachtet bleiben hierbei Beiträge von an der Sonde gebundenen Defekten, da diese nicht mit der hier verwendeten Kontinuumstheorie beschrieben werden können. 


\subsubsection{Punktdefekte}

Punktdefekte umfassen Leerstellen, Zwischengitterplätze und Substitutionsatome. Es handelt sich dabei um nulldimensionale Defekte, die bereits im thermodynamischen Gleichgewicht im Kristall vorhanden sind, aber auch durch Ionenimplantation oder Versetzungsbewegung entstehen können. In der Kontiuumstheorie wird ein Punktdefekt von einem Dehnungsfeld umgeben, dessen Stärke mit der Größe des eingebrachten Defekts verknüpft wird. Da es in einem ternären System wie den MAX-Phasen allein durch Eigendefekte bereits drei verschiedene Leerstellen und Zwischengitteratome zu berücksichtigen gibt, ist es illusorisch, einzelne Punktdefektkonzentrationen durch eine Messung der Verteilungsbreite isolieren zu wollen. Im Folgenden soll deshalb lediglich anhand eines vereinfachten Beispiels in $\mathrm{Ti}_{2} \mathrm{AlN}$ auf die universellen Eigenschaften der durch Punktdefekte erzeugten Feldgradientenverteilung eingegangen werden.

Aus dem Versetzungsfeld

$$
\vec{u}(x, y, z)=\frac{S}{r^{3}} \vec{r} \text { mit } \vec{r}=(x, y, z)
$$

um einen Punktdefekt der Stärke $S$ in einem isotropen Medium ${ }^{a}$ [53] und Gleichung 2.3 kann direkt das Dehnungsfeld

$$
\varepsilon_{i j}(x, y, z)=\frac{S}{r^{3}}\left(\delta_{i j}-3 \frac{x_{i} x_{j}}{r^{2}}\right)
$$

ermittelt werden. Die Defektstärke kann, je nach Größe des Defekts relativ zum ersetzten Volumen, sowohl positiv als auch negativ sein. Obwohl weder die Anisotropie des Wirtsgitters berücksichtigt wird noch Kenntnis über die tatsächlichen Defekte und ihrer relativen Häufigkeit vorliegen, können mit Hilfe von Gleichung 4.4 bereits Aussagen über die Form der EFG-Verteilung getroffen werden.

So ergibt sich anhand der Monte-Carlo-Simulation eine Wahrscheinlichkeitsdichtefunktion $P\left(V_{z z}\right)$, die hervorragend mit einer Lorentz-Verteilung beschrieben werden kann (siehe Abbildung $4.6^{b}$ ). Dies steht im Einklang mit der aus der sogenannten Random-WalkTheorie analytisch vorhergesagten Verteilung des durch Punktdefekte erzeugten EFGs in kubischen Systemen [91]. Der Mittelwert des EFGs verschiebt sich je nach Stärke und Vorzeichen des Defekts.

Die Wahrscheinlichkeitsdichte von $\eta$ verschwindet für $\eta=0$, obwohl die Einzelverteilungen $P\left(V_{x x}\right)$ und $P\left(V_{y y}\right)$ aus Symmetriegründen identisch sind. Hieraus folgt, dass $V_{x x}$ und $V_{y y}$ nicht vollständig unabhängig voneinander sein können, da andernfalls bei $\eta=$ 0 die Wahrscheinlichkeitsdichte maximal wäre. Dieses Verhalten tritt auf, da im Beispiel alle Dehnungen vom gleichen Vorzeichen sind. Würden Defekte mit entgegengesetzten Vorzeichen berücksichtigt, wäre dies nicht der Fall. Aufgrund der Isotropie des Gradientelastischen Tensors in der Basalebene und der fehlenden Vorzugsrichtung des Dehnungsfeldes in dieser Ebene kann $P(\eta)$ als symmetrisch um null angenommen werden. Über die

\footnotetext{
${ }^{a}$ Für anisotrope Medien findet sich kein vergleichbarer Zusammenhang.

${ }^{b} \mathrm{Im}$ gegebenen Beispiel wurde ein Defekt der Stärke $S=-5 \cdot 10^{-31} \mathrm{~m}^{3}$ angenommen, was ungefähr der Stärke einer Leerstelle entspricht. Die Punktdefektdichte wurde mit $10^{25} \mathrm{~m}^{-3}$ angenommen.
} 
(a)

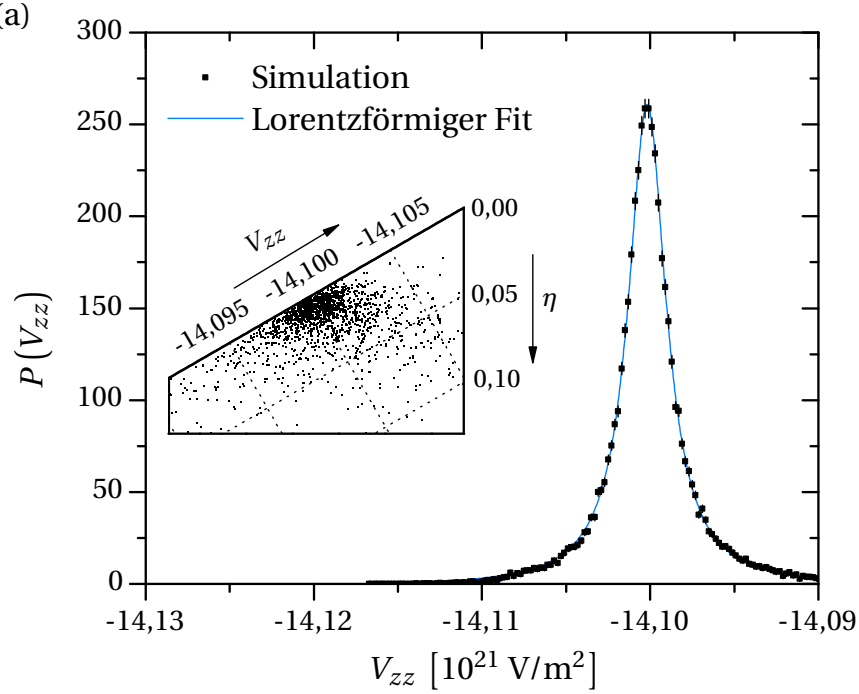

(b)

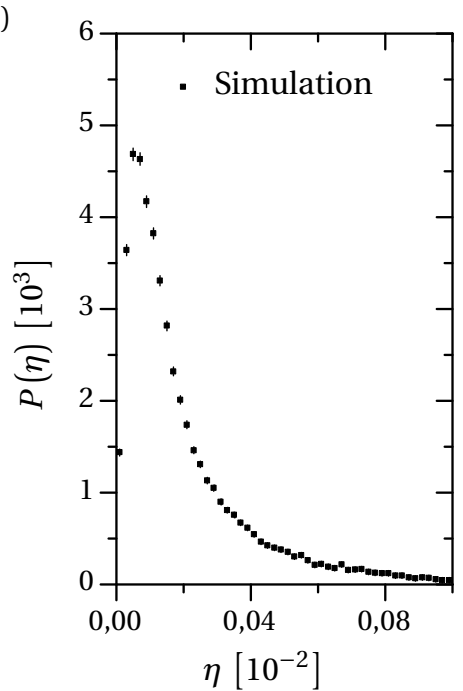

Abbildung 4.6: Wahrscheinlichkeitsdichtefunktion der größten EFG-Tensorkomponente $V_{z z}$ (a) und des Asymmetrieparameters $\eta$ (b) an einem Cd-Kern in $\mathrm{Ti}_{2} \mathrm{AlN}$ mit Punktdefekten der Stärke $S=-5 \cdot 10^{-31} \mathrm{~m}^{3}$ in einer Dichte von $10^{25} \mathrm{~m}^{-3}$. Trotz inadäquater Annahme eines isotropen Spannungsfeldes der Punktdefekte und mangelnder Kenntnis über die tatsächlichen Defektstärken ist es anhand der Simulation möglich, den qualitativen Verlauf zu beschreiben. Die Verteilung von $V_{z z}$ wird hervorragend durch eine Lorentz-Verteilung beschrieben. Gegen null wird die Dichtefunktion von $\eta$ kleiner, da alle auftretenden Dehnungen von gleichem Vorzeichen sind. Eine direkte Korrelation zwischen $V_{z z}$ und $\eta$ (siehe Einschub) ist nicht gegeben.

(a)

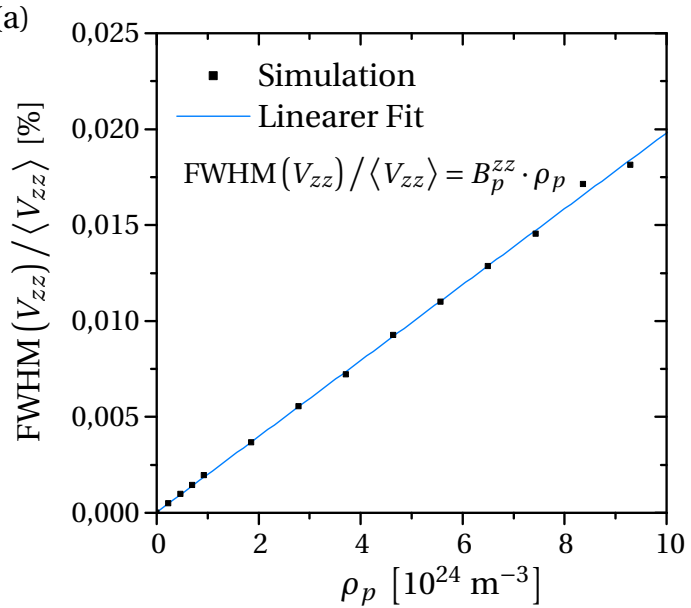

(b)

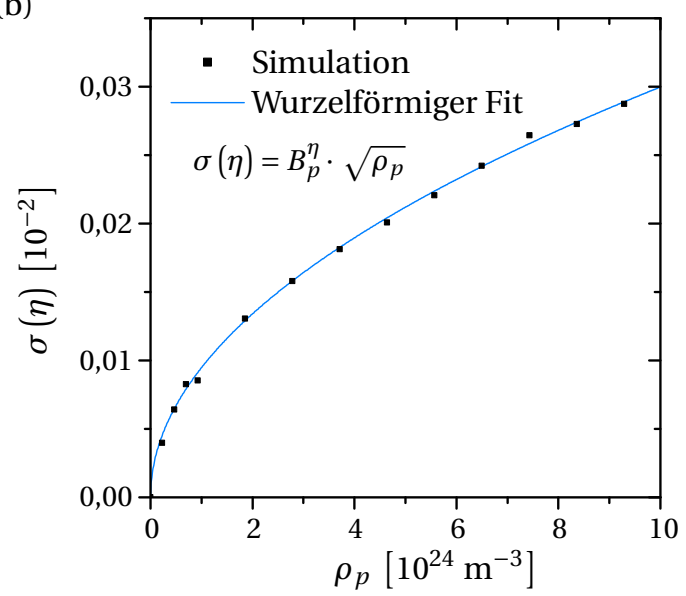

Abbildung 4.7: Hypothetische Verbreiterung von $V_{z z}$ (a) und $\eta$ (b) als Funktion der Punktdefektdichte. Das Spannungsfeld der Defekte entspricht dem Feld in einem isotropen Medium, die Defektstärke beträgt $S=-5 \cdot 10^{-31} \mathrm{~m}^{3}$. Die Halbwertsbreite von $V_{z z}$ steigt linear mit der Defektdichte an, die Verbreiterung von $\eta$ genügt einer Wurzelfunktion. Für eine sinnvolle Angabe defektspezifischer Skalierungsfaktoren $B_{p}^{z z}$ und $B_{p}^{\eta}$ würden das anisotrope Spannungsfeld und reale Defektparameter benötigt. 
Verteilung von $\eta$ finden sich in Referenz [91] keinerlei Aussagen, da hier vermutlich der analytische Ansatz versagt. Eine Korrelation zwischen $V_{z z}$ und $\eta$ ist im Czjzek-Diagramm nicht ersichtlich.

Der Einfluss der Transformation ins Hauptachsensystem äußert sich in einer hier kaum sichtbaren Asymmetrie der Verteilung $P\left(V_{z z}\right)$. Die Form der Verteilung wird dabei maßgeblich durch die Normalkomponenten der Dehnung bestimmt; erst in der Umgebung von reinen Scherdehnungen wird diese Transformation relevant.

Eine Analyse der Verteilungsbreiten als Funktion der Defektdichte erfolgt für $V_{z z}$ sinnvollerweise anhand der Halbwertsbreite, da es sich um eine Lorentz-Verteilung handelt. Die Verbreiterung von $V_{z z}$ steigt linear mit der Punktdefektdichte $\rho_{p}$ an (siehe Abbildung 4.7a). Dies kann anschaulich dadurch erklärt werden, dass der mittlere Abstand $r$ zwischen Sonde und Punktdefekt mit $\rho_{p}^{-1 / 3}$ skaliert, gleichzeitig aber das Dehnungsfeld mit $1 / r^{3}$ abnimmt. Beide Potenzen heben sich schließlich auf, womit die mittlere Dehnung und die Verbreiterung des Feldgradienten proportional zu $\rho_{p}$ sind.

Im Falle des Asymmetrieparameters wird die numerisch erhaltene Standardabweichung $\sigma(\eta)$ als Maß der Verbreiterung herangezogen. Hier wird qualitativ ein wurzelförmiger Verlauf beobachtet (siehe Abbildung 4.7b). Eine einfache Erklärung für diesen Verlauf existiert vermutlich nicht, da für $\eta$ die Differenz der nicht vollständig unabhängigen Komponenten $V_{x x}$ und $V_{y y}$ relevant ist.

Die bereits angesprochene Problematik aus unbekannter Defektstärke und fehlendem anisotropen Dehnungsfeld verhindert eine sinnvolle Quantifizierung der Skalierungsfaktoren. Davon unberührt bleibt die Erkenntnis, dass die durch Punktdefekte hervorgerufene EFG-Verteilungen immer einer Lorentz-Verteilung entspricht. Die Halbwertsbreite dieser Verteilung steigt immer linear mit der Punktdefektdichte an.

\subsubsection{Versetzungen}

Versetzungen lassen sich in Stufen- und Schraubenversetzungen oder deren Mischformen unterteilen. Es handelt sich um eindimensionale Defekte, die bereits im Kristallwachstum entstehen oder durch Spannungen im Material erzeugt werden. Versetzungen werden von einem Dehnungsfeld umgeben, dessen Eigenschaften von Burgersvektor und Charakter der Versetzung abhängen. Da in den MAX-Phasen so gut wie alle Versetzungen in der Basalebene liegen und den identischen Burgersvektor von $1 / 3\langle 11 \overline{2} 0\rangle$ aufweisen [30], lässt sich unter der Annahme gleich häufig auftretender Stufen- und Schraubenversetzungen [13] die Feldgradientenverteilung quantitativ mit der Versetzungsdichte, d. h. mit der Versetzungslänge pro Volumen, in Verbindung bringen. Wären mehr Gleitsysteme aktiv, wäre diese Analyse erschwert, da Kenntnis über die relative Häufigkeit der beteiligten Gleitsysteme vonnöten wäre. Im Folgenden wird zunächst die Feldgradientenverteilung in der Umgebung reiner Stufenversetzungen untersucht, die Untersuchung für Schraubenversetzung schließt sich daran an.

\section{Stufenversetzungen}

Das Dehnungsfeld um eine Stufenversetzung lässt sich in anisotropen Medien analytisch beschreiben [54]. Der zugehörige Dehnungstensor ist für eine Versetzung in der Basalebe- 
ne mit Versetzungslinie entlang der $y$-Achse von der Form

$$
\varepsilon_{i j}(x, z)=\left(\begin{array}{ccc}
\varepsilon_{x x} & 0 & \varepsilon_{x z} \\
& 0 & 0 \\
& & \varepsilon_{z z}
\end{array}\right) .
$$

Die zugehörigen Komponenten sind in Abhang B aufgeführt. Die Länge des Burgersvektors entspricht der Gitterkonstante $a$.

Die Monte-Carlo-Simulation einer zufälligen Anordnung solcher Defekte erzeugt eine Wahrscheinlichkeitsdichtefunktion $P\left(V_{z z}\right)$, die gut mit einer Gauß-Verteilung beschrieben werden kann (siehe Abbildung 4.8). Der Mittelwert dieser Verteilung ist nicht verschoben, da sich das Dehnungsfeld einzelner Versetzungen zu gleichen Teilen aus positiven und negativen Dehnungen zusammensetzt. Auf eine Beschreibung mit einer komplexeren Funktion wie beispielsweise einem Voigt-Profil wird verzichtet, um die Zahl der Freiheitsgrade möglichst gering zu halten. Die durch die Transformation ins Hauptachsensystem eingebrachte Asymmetrie der Verteilung ist wie bei den Punktdefekten vernachlässigbar klein.

Die Wahrscheinlichkeitsdichte von $\eta$ ist maximal für $\eta=0$ und kann aufgrund der Isotropie von $S_{m n}$ in der Basalebene und der fehlende Vorzugsrichtung der Versetzung in dieser Ebene als symmetrisch um $\eta=0$ angenommen werden. Anhand der Dichte lässt sich keine Korrelation zwischen $V_{x x}$ und $V_{y y}$ erkennen. Das zugehörige Czjzek-Diagramm zeigt Wertepaare von $V_{z z}$ und $\eta$, auch hier lässt sich keine Korrelation erkennen.

Im Vergleich zu den drei analytischen Näherungen aus Referenz [91] fällt auf, dass diese drei Ansätze entweder den Bereich kleiner Änderungen extrem unterschätzen ${ }^{a}$ oder den Bereich großer Änderung überschätzen ${ }^{b c}$. Die hier zusätzlich durchgeführte Transformation ins Hauptachsensystem ist nicht ursächlich für die Unterschiede. Vielmehr führen die für eine analytische Lösung nötigen Näherungen zu systematischen Fehlern in der Beschreibung der Verteilung.

Eine Analyse der Verteilungsbreite als Funktion der Defektdichte erfolgt anhand der Standardabweichung von $V_{z z}$ und $\eta$. Beide Verteilungsbreiten wachsen wurzelförmig mit der Defektdichte an (siehe Abbildung 4.9). Der wurzelförmige Verlauf ist eine direkte Konsequenz aus der $1 / r$-Abhängigkeit des Dehnungsfeldes um eine Versetzung und der $\sqrt{1 / \rho}$ Abhängigkeit des mittleren Abstandes zwischen Versetzung und Sonde. Die Verbreiterung von $V_{z z}$ und $\eta$ lässt sich mit Hilfe der Konstanten $B_{e}^{z z}$ und $B_{e}^{\eta}$ durch

$$
\sigma\left(V_{z z}\right) /\left\langle V_{z z}\right\rangle=B_{e}^{z z} \cdot \sqrt{\rho_{e}}
$$

\footnotetext{
${ }^{a}$ Im sogenannten Poisson-Modell [91] wird nur die Dehnung der jeweils nächsten Versetzung zur Sonde berücksichtigt. Dieser Dehnung entgegenwirkende Beiträge anderer, weiter entfernter Versetzungen werden ignoriert; Bereiche kleiner Änderungen des EFGs werden somit unterschätzt.

${ }^{b}$ Die sogenannte Random-Walk-Theorie [91] erfordert zur Beschreibung von Versetzungen einen willkürlich gewählten Abschneideparameter, da sonst ein zu lösendes Integral divergiert. Des Weiteren wird implizit angenommen, dass jede Versetzung abhängig vom Abstand zur Sonde entweder mit einem fixen Anteil oder gar nicht zum EFG beiträgt.

${ }^{c}$ Der dritte Ansatz besteht aus einer willkürlich gewählten Verteilung, die das gleiche asymptotische Verhalten und die gleiche mittlere absolute Abweichung aufweist wie die Lösung des Poisson-Modells [91]. Dieser Ansatz soll den Fehler des Poisson-Modells in der Beschreibung kleiner Änderungen beheben.
} 
(a)

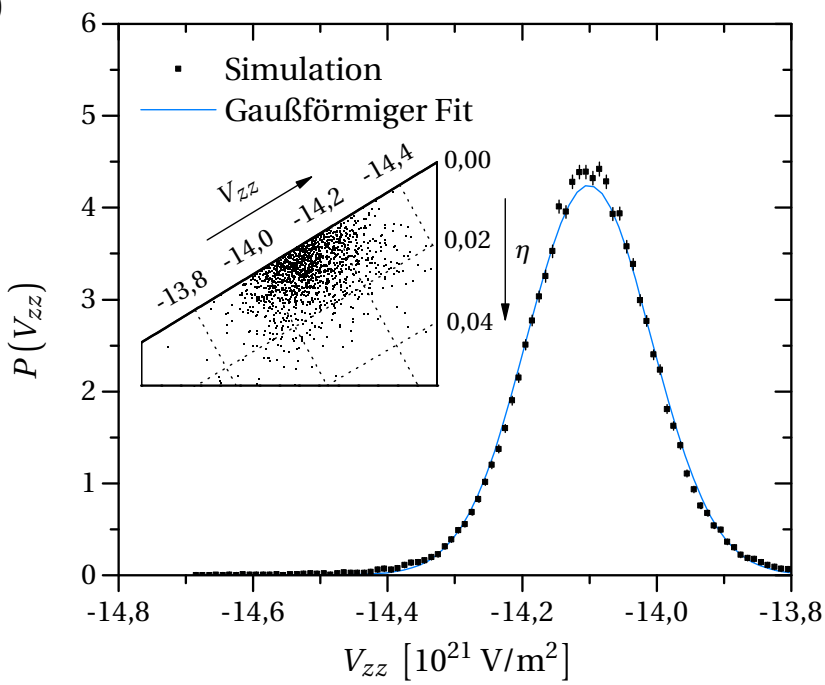

(b)

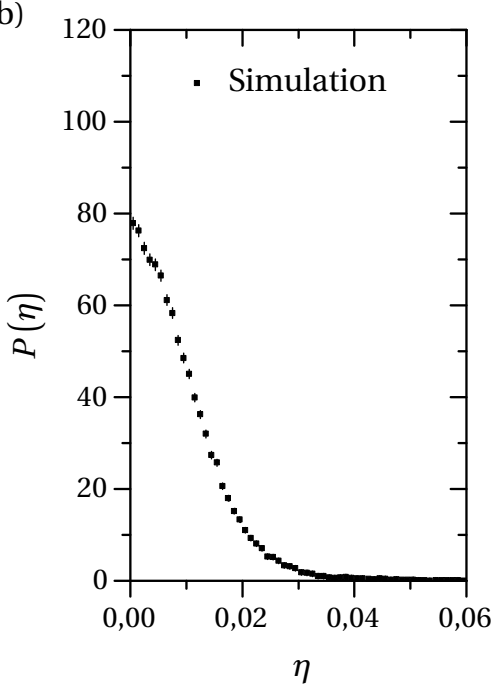

Abbildung 4.8: Simulierte Wahrscheinlichkeitsdichtefunktion der größten EFG-Tensorkomponente $V_{z z}$ (a) und des Asymmetrieparameters $\eta$ (b) an einem Cd-Kern in $\mathrm{Ti}_{2} \mathrm{AlN}$ mit $5 \cdot 10^{14} \mathrm{~m}^{-2}$ zufällig in der Basalebene verteilten Stufenversetzungen. Die Standardabweichung von $V_{z z}$ beträgt in Einheiten des Mittelwertes 0,66(2)\%, die von $\eta$ 0,0106(3). Die Verteilung von $V_{z z}$ wird gut durch eine Gauß-Verteilung beschrieben. Eine direkte Korrelation zwischen $V_{z z}$ und $\eta$ (siehe Einschub) ist nicht gegeben.

(a)

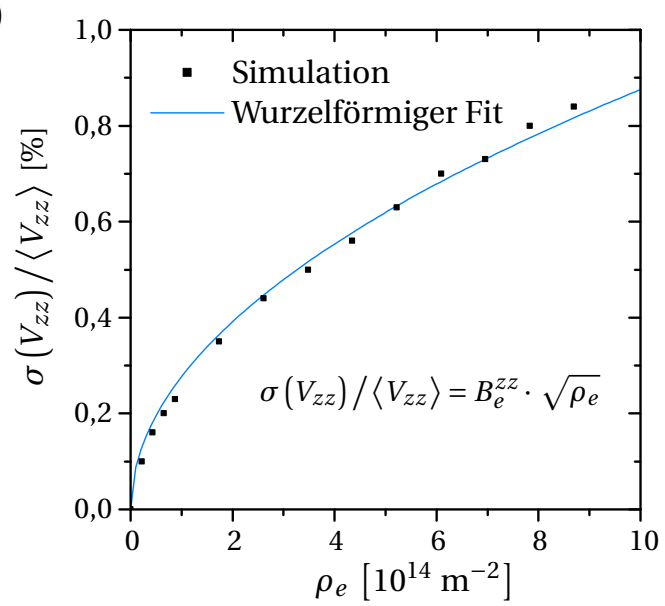

(b)

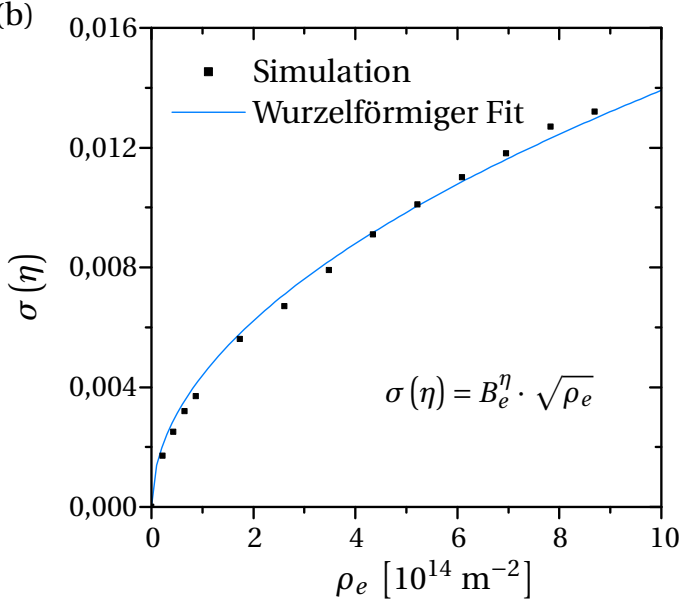

Abbildung 4.9: Standardabweichung von $V_{z z}$ (a) und $\eta$ (b) an der Cd-Sonde in $\mathrm{Ti}_{2} \mathrm{AlN}$ als Funktion der Stufenversetzungsdichte $\rho_{e}$. Die Simulation zeigt für beide Abhängigkeiten einen wurzelförmigen Verlauf, der eine direkte Konsequenz der $1 / r$-Abhängigkeit des Dehnungsfeldes um eine Versetzung und der $\sqrt{1 / \rho}$-Abhängigkeit des mittleren Abstandes zwischen Versetzung und Sonde ist. Die entsprechenden Skalierungsfaktoren lauten für $\mathrm{Ti}_{2} \mathrm{AlN} B_{e}^{z z}=2,77(4) \cdot 10^{-10} \mathrm{~m}$ und $B_{e}^{\eta}=4,40(6) \cdot 10^{-10} \mathrm{~m}$. Für Schraubenversetzungen ergibt sich qualitativ der gleiche Verlauf, lediglich die Skalierungsfaktoren sind verschieden. 
Tabelle 4.3: Kopplungskonstanten $B_{e}^{z z}$ und $B_{e}^{\eta}$ sowie $B_{s}^{z z}$ und $B_{e}^{\eta}$, die die (relative) Verbreiterung von $V_{z z}$ und $\eta$ an die Quadratwurzel der Stufen- bzw. Schraubenversetzungsdichte koppeln. Die Verbreiterung von $V_{z z}$ durch Schraubenversetzungen ist vernachlässigbar klein.

\begin{tabular}{ccccc}
\hline Wirtsgitter & $B_{e}^{z z}\left[10^{-10} \mathrm{~m}\right]$ & $B_{e}^{\eta}\left[10^{-10} \mathrm{~m}\right]$ & $B_{s}^{z z}\left[10^{-10} \mathrm{~m}\right]$ & $B_{s}^{\eta}\left[10^{-10} \mathrm{~m}\right]$ \\
\hline $\mathrm{Nb}_{2} \mathrm{AlC}$ & $4,2(4)$ & $3,1(3)$ & $0,069(5)$ & $5,6(4)$ \\
$\mathrm{Ti}_{2} \mathrm{AlC}$ & $2,2(2)$ & $1,1(1)$ & $0,0045(5)$ & $1,5(1)$ \\
$\mathrm{Ti}_{2} \mathrm{AlN}$ & $2,77(4)$ & $4,40(6)$ & $0,051(4)$ & $5,8(1)$ \\
$\mathrm{Ti}_{2} \mathrm{InC}$ & $2,9(1)$ & $2,9(1)$ & $0,030(2)$ & $3,9(2)$ \\
\hline
\end{tabular}

und

$$
\sigma(\eta)=B_{e}^{\eta} \cdot \sqrt{\rho_{e}}
$$

quantifizieren. Die entsprechenden Konstanten sind für die hier untersuchten MAX-Phasen in Tabelle 4.3 aufgelistet.

\section{Schraubenversetzungen}

Das Dehnungsfeld um eine Schraubenversetzung in der Basalebene in einem anisotropen Medium [54] weist die Form

$$
\varepsilon_{i j}(x, z)=\left(\begin{array}{ccc}
0 & \varepsilon_{x y} & 0 \\
& 0 & \varepsilon_{y z} \\
& & 0
\end{array}\right)
$$

auf. Die zugehörigen Komponenten sind in Anhang B aufgeführt. Da es sich hierbei um ein Dehnungsfeld handelt, das sich im Hauptachsensystem des Feldgradienten allein aus Scherdehnungen zusammensetzt, ist die Diagonalisierung des resultierenden EFGTensors essentiell. Bliebe diese Transformation unberücksichtigt, würden sich weder $V_{z z}$ noch $\eta$ ändern, da Scherungen aufgrund der Struktur des Gradient-elastischen Tensors in den MAX-Phasen keinen Einfluss auf die Normalkomponenten haben. Die Länge des Burgersvektors entspricht, wie auch bei den Stufenversetzungen, der Gitterkonstante $a$.

Die Monte-Carlo-Simulation zeigt, dass die zufällige Anordnung solcher Defekte eine asymmetrische Wahrscheinlichkeitsdichte $P\left(V_{z z}\right)$ hervorruft (siehe Abbildung 4.10). Das Maximum der Verteilung ist durch den ursprünglichen EFG $V_{z z}^{0}$ festgelegt. Im Vergleich zu den Stufenversetzungen fällt die Verteilungsbreite zwei Größenordnungen kleiner aus.

Die Wahrscheinlichkeitsdichte von $\eta$ zeigt einen zu den Stufenversetzungen vergleichbaren Verlauf, der als symmetrisch um null angenommen werden kann. Das Maximum der Verteilung befindet sich bei $\eta=0$, es gibt also keinen Hinweis auf eine Abhängigkeit zwischen $V_{x x}$ und $V_{y y}$. Auch zwischen $V_{z z}$ und $\eta$ zeigt sich keine Korrelation, lediglich der Wertebereich für $V_{z z}$ ist stark eingeschränkt.

Der im Vergleich zu den Stufenversetzungen grundlegend andere Verlauf der Verteilung $P\left(V_{z z}\right)$ ist, wie oben schon angedeutet, auf den speziellen Einfluss reiner Scherdehnungen auf den schon vorhandenen EFG zurückzuführen. In kubischen Systemen besteht diese 

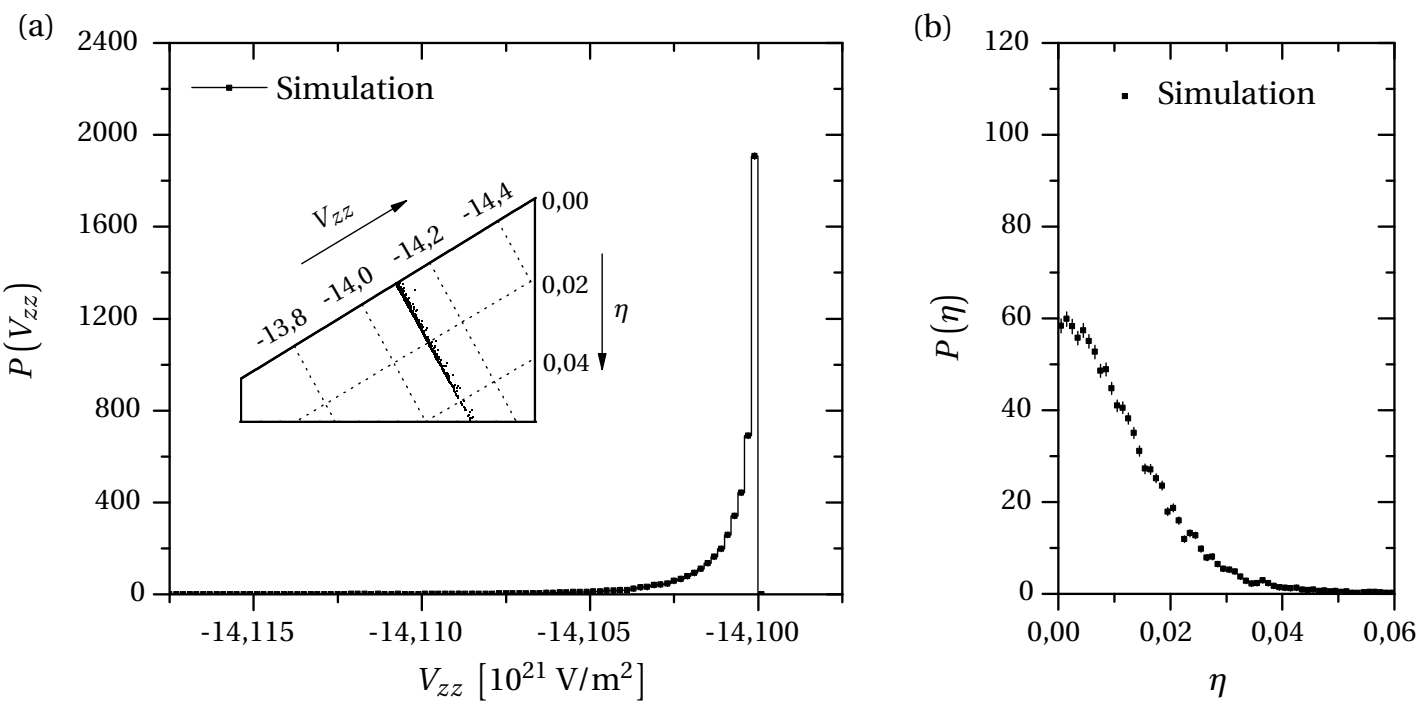

Abbildung 4.10: Simulierte Wahrscheinlichkeitsdichte der größten EFG-Tensorkomponente $V_{z z}$ (a) und des Asymmetrieparameters $\eta$ (b) an einem Cd-Kern in Ti ${ }_{2} \mathrm{AlN}$ mit $5 \cdot 10^{14} \mathrm{~m}^{-2}$ zufällig in der Basalebene verteilten Schraubenversetzungen. Die Standardabweichung von $V_{z z}$ beträgt in Einheiten des Mittelwertes 0,010(1)\%, die von $\eta$ 0,0139(2). Die Verteilung von $V_{z z}$ ist asymmetrisch und wird vollständig durch die Transformation ins Hauptachsensystem hervorgerufen. Eine direkte Korrelation zwischen $V_{z z}$ und $\eta$ (siehe Einschub) ist nicht gegeben, lediglich der Wertebereich von $V_{z z}$ ist eingeschränkt. Die Verteilungsbreiten von $V_{z z}$ und $\eta$ zeigen die gleiche wurzelförmige Abhängigkeit zur Defektdichte wie im Falle der Stufenversetzungen, die zugehörigen Konstanten lauten $B_{s}^{z z}=0,051(4) \cdot 10^{-10} \mathrm{~m}$ und $B_{s}^{\eta}=5,78(9) \cdot 10^{-10} \mathrm{~m}$.

Diskrepanz nicht [91], da hier kein intrinsischer EFG vorhanden ist. Der entscheidende Unterschied zwischen Systemen mit und ohne intrinsischem Feldgradienten besteht hierbei in der ungleichen Auswirkung der Scherkomponente $\varepsilon_{y z}$ auf den EFG im Hauptachsensystem. So bildet der intrinsisch vorhandene EFG eine Vorzugsrichtung im System, die durch die Scherkomponente nur leicht verändert wird. Diese Vorzugsrichtung existiert im kubischen System nicht; erst die Scherung legt diese Richtung fest, wodurch die Eigenwerte des EFG-Tensors maximal beeinflusst werden. Gleiches gilt für die Wirkung einer Schraubenversetzung auf $\eta$, die trotz fehlender Normalkomponente des Dehnungsfeldes relativ groß ist. Hier beeinflusst die Scherkomponenten $\varepsilon_{x y}$ die beiden Komponenten $V_{x x}$ und $V_{y y}$ maximal, da aufgrund der Axialsymmetrie des Feldgradienten in der $x$ - $y$-Ebene keine Vorzugsrichtung vorliegt.

Die Abhängigkeit der Verteilungsbreiten von der Schraubenversetzungsdichte $\rho_{s}$ zeigt wie im Falle der Stufenversetzungen einen wurzelförmigen Verlauf, der auf die $1 / r$-Abhängigkeit des Dehnungsfeldes zurückzuführen ist. Analog zu Gleichungen 4.6 und 4.7 lässt sich dieser Zusammenhang über materialspezifische Konstanten $B_{s}^{z z}$ und $B_{s}^{\eta}$ quantifizieren. Die entsprechenden Konstanten der MAX-Phasen sind in Tabelle 4.3 aufgelistet. Beim Vergleich mit den für Stufenversetzungen relevanten Konstanten $B_{e}^{z z}$ und $B_{e}^{\eta}$ fällt auf, dass Schraubenversetzungen zur Verbreiterung von $V_{z z}$ vernachlässigbar wenig beitragen. 


\subsubsection{Korngrenzen und andere höherdimensionale Defekte}

Bereiche einer Korngrenze können im Hinblick auf den elektrischen Feldgradienten als stark gestörte Umgebung betrachtet werden, die mit der eigentlichen Kistallstruktur nur noch wenig gemein hat. Eine Beschreibung dieser Bereiche mit Dehnungsfeldern ist nicht möglich. Einzig Kleinwinkelkorngrenzen könnten kontinuumstheoretisch als eine Aneinanderreihung von Versetzungen beschrieben werden. Unabhängig von diesem konzeptionellen Problem ist anzunehmen, dass die durch Korngrenzen verursachte Verbreiterung des Feldgradienten mit dem Verhältnis aus Oberfläche und Volumen der Kristallite verknüpft ist. Aus dieser Annahme ergibt sich ein zur inversen Korngröße proportionaler Beitrag, der erst für nanokristalline Materialien signifikant wird [191].

Andere höherdimensionale Defekte wie beispielsweise Einschlüsse im Material oder Ausscheidungen tragen nicht zur Verbreiterung des ursprünglichen Feldgradienten bei. Sonden in einer solch vollständig fremden Umgebung weisen vielmehr einen neuen, vom ursprünglichen EFG abweichenden Feldgradienten auf. Risse und Delaminationen bilden im obigen Sinne lediglich neue Oberflächen, tragen selbst also praktisch nicht zu einer Verbreiterung bei; etwaige bei deren Entstehung erzeugte Punkt- oder Liniendefekte hingegen schon.

\subsubsection{Elastische Verbreiterung}

Die Änderung des Feldgradienten durch eine uniaxiale, rein elastische Belastung wird für Einkristalle im Rahmen der linear-elastischen Theorie durch Gleichung 2.5 eindeutig beschrieben. Beim Übergang hin zu polykristallinen Materialien wird diese Eindeutigkeit aufgehoben, da eine Verteilung unterschiedlicher Kornorientierungen relativ zur uniaxialen Last eine Verteilung des Feldgradienten erzeugt. Diese elastische Verbreiterung wird im Folgenden unter idealisierten Bedingungen zunächst im Koordinatensystem von $V_{i j}^{0}$ analytisch, anschließend unter Berücksichtigung der Transformation ins Hauptachsensystem numerisch quantifiziert.

Die angelegte uniaxiale Spannung wird mit $\sigma_{u}$ gekennzeichnet und idealisiert als homogen betrachtet (Reuss'scher Ansatz [192]). Da es sich um polykristalline Proben handelt, kann diese Last ohne Beschränkung der Allgemeinheit entlang einer z-Achse, beispielsweise senkrecht zur Detektorebene, angelegt werden. Der zugehörige Spannungstensor lautet

$$
\sigma_{i j}=\left(\begin{array}{ccc}
0 & 0 & 0 \\
0 & 0 & 0 \\
0 & 0 & -\sigma_{u}
\end{array}\right) .
$$

Die Orientierung dieser uniaxialen Last relativ zur Kornorientierung, das heißt auch relativ zur Orientierung von $V_{i j}^{0}$, wird durch eine Transformation des Spannungstensors mit Hilfe der zwei Euler'schen Winkel $\varphi$ und $\theta$ verändert. Die Rotation um den dritten Euler'schen Winkel $\psi$ ist irrelevant, da es sich um eine uniaxiale Last handelt. Es ergibt sich ein winkelabhängiger Spannungstensor $\sigma_{i j}\left(\sigma_{u}, \varphi, \theta\right)$, der zusammen mit Gleichung 2.5 den allgemeinen EFG-Tensor $V_{i j}\left(\sigma_{u}, \varphi, \theta\right)$ bildet. Dieser allgemeine Tensor berücksichtigt sowohl die Stärke der uniaxialen Spannung als auch die Orientierung des Korns relativ zur Kompressionsachse. 
Eine Untersuchung der Winkelabhängigkeit zeigt, dass die größte Komponente des EFG-Tensors, $V_{z z}$, unabhängig vom ersten Euler'schen Winkel ist, wenn die Gradientelastischen Konstanten $C_{31}$ und $C_{32}$ identisch sind und $C_{34}, C_{35}$ und $C_{36}$ verschwinden. Dies trifft auf alle hier untersuchten Systeme zu (vgl. Abbildung 4.1) ${ }^{a}$. Die Winkelabhängigkeit dieser Komponente kann so auf

$$
V_{z z}\left(\sigma_{u}, \theta\right)=V_{z z}^{0}-\left(C_{31} \sin ^{2} \theta+C_{33} \cos ^{2} \theta\right) \sigma_{u}
$$

vereinfacht werden. Ist zusätzlich die Verteilungsfunktion der Kornorientierungen bekannt, kann hieraus die Verteilungsfunktion von $V_{z z}$ analytisch berechnet werden. Die Transformation des EFG-Tensors ins Hauptachsensystem bleibt hierbei zunächst unberücksichtigt.

Im Folgenden wird diese Orientierungsdichteverteilungsfunktion ${ }^{b}$ als isotrop angenommen. Die zugehörige Wahrscheinlichkeitsdichte in Abhängigkeit vom Euler'schen Winkel $\theta \in[0, \pi]$ lautet $p(\theta)=1 / 2 \sin \theta^{c}$. Diese idealisierte Annahme führt je nach Stärke der Textur zu einem systematischen Fehler in der Beschreibung der EFG-Verteilungsfunktion, der aufgrund mangelnder Kenntnis über die tatsächliche Orientierungsdichteverteilungsfunktion in Kauf genommen werden muss. Da $V_{z z}\left(\sigma_{u}, \theta\right)$ eine gerade Funktion mit Periode $\pi$ ist, das heißt $V_{z z}$ symmetrisch um $\pi / 2$ ist, genügt es, das Intervall $\theta \in[0, \pi / 2]$ zu betrachten. Innerhalb dieses Intervalls verhält sich $V_{z z}$ monoton, was die weitere Rechnung vereinfacht, da sich für monotone Größen die Wahrscheinlichkeitsdichte $P\left(V_{z z}\right)$ durch

$$
P\left(V_{z z}\right)= \begin{cases} \pm p\left[\theta\left(V_{z z}\right)\right] \frac{\mathrm{d} \theta\left(V_{z z}\right)}{\mathrm{d} V_{z z}} & \text { für } V_{z z} \in\left[V_{z z}\left(\sigma_{u}, 0\right), V_{z z}\left(\sigma_{u}, \pi / 2\right)\right] \\ 0 & \text { sonst }\end{cases}
$$

berechnen lässt. Für monoton steigende $V_{z z}(\theta)$ gilt das positive Vorzeichen, für fallende das negative. Durch Umstellen von Gleichung 4.10 und normieren von $p(\theta)$ auf das Intervall zwischen 0 und $\pi / 2$ folgt

$$
p\left[\theta\left(V_{z z}\right)\right] \frac{\mathrm{d} \theta\left(V_{z z}\right)}{\mathrm{d} V_{z z}}=\frac{1}{2\left(C_{31}-C_{33}\right) \sigma_{u}} \sec \left[\frac{1}{2} \arccos \frac{\sigma_{u}\left(C_{31}+C_{33}\right)-2 V_{z z}+2 V_{z z}^{0}}{\sigma_{u}\left(C_{31}-C_{33}\right)}\right] .
$$

Die entsprechende Verteilung ist für $\mathrm{Ti}_{2} \mathrm{AlN}$ in Abbildung 4.11(a) gezeigt. Es handelt sich um eine normierte Verteilung, deren Träger durch die extremalen Orientierungen der Last beschränkt ist. Für Orientierungen in der Basalebene divergiert die Wahrscheinlichkeitsdichte, da sich die Dichte $p(\theta)$ in diesem Bereich nur minimal ändert.

\footnotetext{
${ }^{a}$ Dieses doch sehr spezielle Argument scheint auf den ersten Blick bei den Komponenten $V_{x x}$ und $V_{y y}$ nicht zu greifen. Hier spielt $\phi$ also scheinbar eine Rolle, was aber aber im Widerspruch zu jenen hexagonalen Systemen steht, die sich in der Basalebene isotrop verhalten. Die Lösung dieses Problems liegt in der hier ignorierten Transformation ins Hauptachsensystem. Erst die Eigenwerte des EFG-Tensors sind für diese Materialien unabhängig von $\phi$.

${ }^{b}$ Prinzipiell ließe sich die Orientierungsdichteverteilungsfunktion anhand einer Polfigur mittels Röntgendiffraktometrie bestimmen. Vergleichbare Messungen wurden in dieser Arbeit jedoch nicht durchgeführt, womit die Orientierung der Körner idealisiert als regellos angenommen wird.

${ }^{c}$ Vgl. Funktionaldeterminante in Kugelkoordinaten.
} 
Der Mittelwert von $V_{z z}$ ändert sich linear mir der Spannung gemäß

$$
\left\langle V_{z z}\right\rangle=V_{z z}^{0}-\frac{2 C_{31}+C_{33}}{3} \cdot \sigma_{u},
$$

womit für dessen relative Änderung

$$
\frac{\mathrm{d} \ln \left\langle V_{z z}\right\rangle}{\mathrm{d} \sigma_{u}}=-\frac{2 C_{31}+C_{33}}{3 V_{z z}^{0}}=: A_{u}^{z z}
$$

gilt. Dies entspricht genau einem Drittel der Änderung, wie sie unter einem isostatischem Druck $p$ erwartet wird.

Der gleiche lineare Zusammenhang ergibt sich auch für die Verteilungsbreite, allerdings lässt sich hier die Standardabweichung $\sigma\left(V_{z z}\right)$ nur numerisch bestimmen. Es folgt für die Spannungsabhängigkeit der Standardabweichung in Einheiten des Mittelwertes

$$
\frac{\sigma\left(V_{z z}\right)}{\left\langle V_{z z}\right\rangle}=B_{u}^{z z} \cdot \sigma_{u}
$$

mit der materialspezifischen Proportionalitätskonstante $B_{u}^{z z}$.

Die Wahrscheinlichkeitsdichte $P(\eta)$ kann für Systeme, die sich isotrop in der Basalebene verhalten, auf die gleiche Weise berechnet werden. Ohne Beschränkung der Allgemeinheit kann hier der Euler'sche Winkel $\phi=0$ gesetzt werden (siehe oben), womit sich beispielsweise für $\mathrm{Ti}_{2} \mathrm{AlN}$ die Dichte zu

$$
P(\eta)=\frac{\left(C_{11}-C_{12}\right)\left(C_{33}+V_{z z}^{0} / \sigma_{0}\right)}{2\left[C_{11}-C_{12}-|\eta|\left(C_{31}-C_{33}\right)\right]^{3 / 2}\left[C_{11}-C_{12}-|\eta|\left(C_{31}+V_{z z}^{0} / \sigma_{0}\right)\right]^{1 / 2}}
$$

ergibt (siehe Abbildung 4.11(b)). Maximale $\eta$ entstehen bei Belastungen in der Basalebene, die entsprechende Wahrscheinlichkeitsdichte divergiert. Aufgrund der fehlenden Vorzugsrichtung in der Basalebene wird der Verlauf als symmetrisch um null angenommen. Der Mittelwert der Verteilung liegt somit bei null. Es handelt sich also bei der elastischen Verbreiterung um eine Situation, in der - im Terminus von Butz - die Axialsymmetrie erhalten bleibt, wenngleich das Maximum nicht bei $\eta=0$ liegt (siehe Kapitel 3.1.4). Die Standardabweichung $\sigma(\eta)$ ist wie $\sigma\left(V_{z z}\right)$ analytisch nicht zugänglich, steigt aber linear mit der Spannung an. Es gilt

$$
\sigma(\eta)=B_{u}^{\eta} \cdot \sigma_{u}
$$

Für homogene Spannungen folgt eine eindeutige Korrelation zwischen $V_{z z}$ und $\eta$. Dies wird nachfolgend bei der numerischen Analyse deutlich.

Die numerische Behandlung der elastischen Verbreiterung basiert wie auch der analytische Ansatz auf der Annahme einer regellosen Kornorientierung und der eines homogenen Spannungsfeldes. Ausgehend vom orientierungs- und spannungsabhängigen EFG-Tensor $V_{i j}\left(\sigma_{u}, \varphi, \theta\right)$ im ursprünglichen Koordinatensystem, kann durch die numerische Berechnung der Eigenwerte dieses Tensors die Verteilung von $V_{z z}$ und $\eta$ im Hauptachsensystem 
(a)

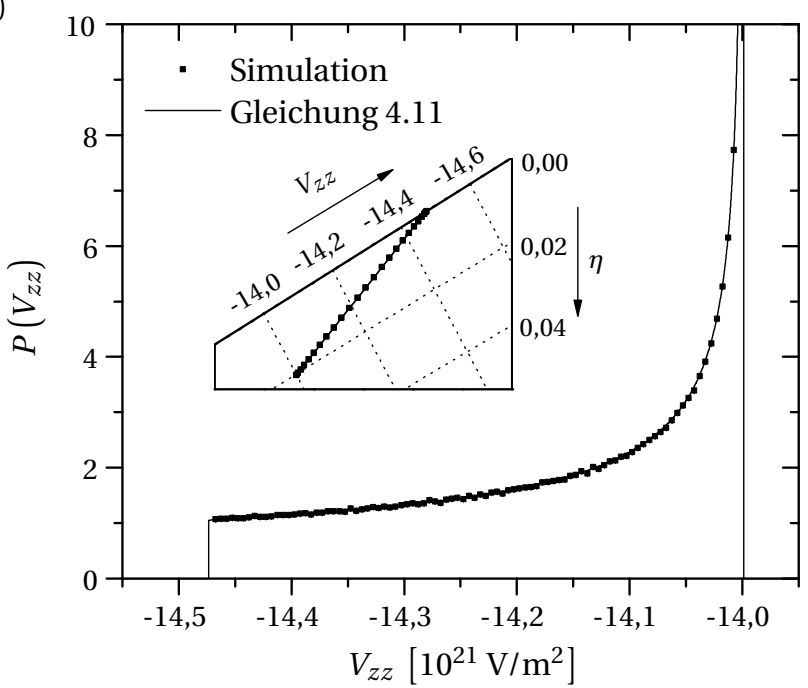

(b)

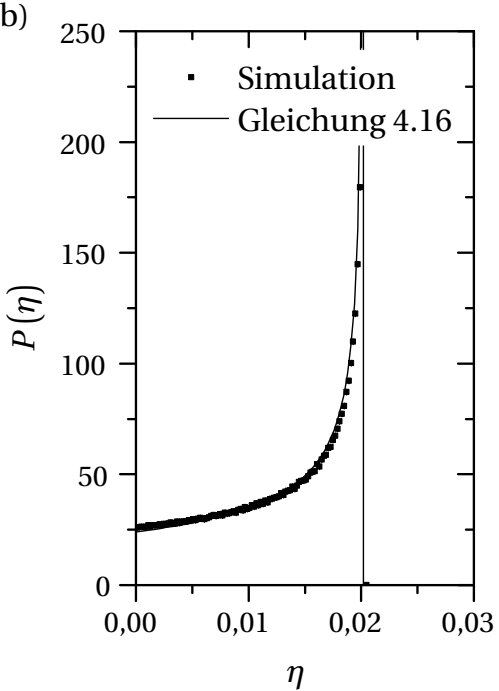

Abbildung 4.11: Dichtefunktion von $V_{z z}$ (a) und $\eta$ (b) an der Cd-Sonde in uniaxial belastetem polykristallinen $\mathrm{Ti}_{2} \mathrm{AlN}$. Für die verwendete Last $\sigma_{u}=1,64 \mathrm{GPa}$ ergibt sich eine Verteilung von $V_{z z}$, deren Mittelwert und Standardabweichung bei $-14,16 \cdot 10^{21} \mathrm{~V} / \mathrm{m}^{2}$ bzw. 1\% liegen. Die Verteilung von $\eta$ wird als symmetrisch um null angenommen. Die zugehörige Standardabweichung beträgt 0,015. Analytische und simulierte Beschreibung zeigen einen nahezu identischen Verlauf. Die Korrelation zwischen $V_{z z}$ und $\eta$ wird im Czjzek-Diagramm (Einschub) in Form einer Geraden deutlich.

(a)

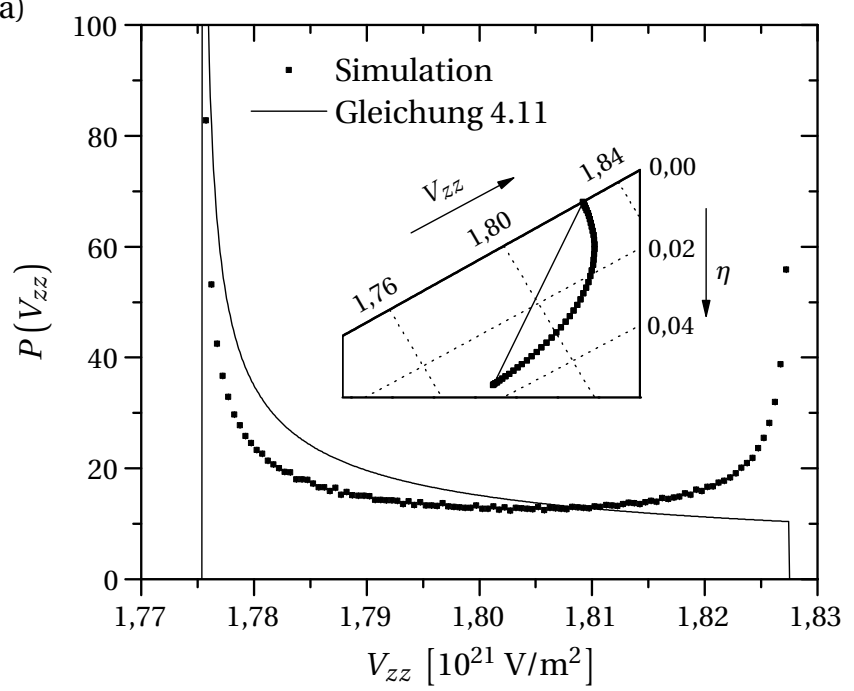

(b)

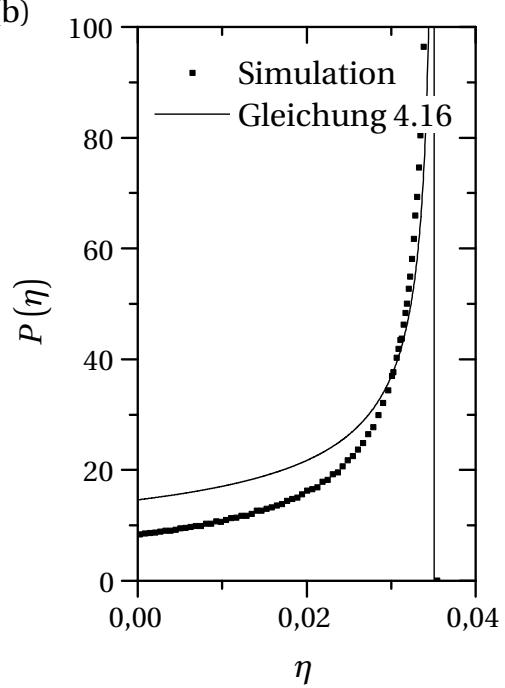

Abbildung 4.12: Dichtefunktion von $V_{z z}$ (a) und $\eta$ (b) an der Cd-Sonde in mit 0,65 GPa uniaxial belastetem polykristallinen Titan. Mittelwert und Standardabweichung von $V_{z z}$ betragen $1,80 \cdot 10^{21} \mathrm{~V} / \mathrm{m}^{2}$ bzw. $1 \%$. Die Standardabweichung von $\eta$ beträgt 0,028 . Anders als bei $\mathrm{Ti}_{2} \mathrm{AlN}$ weicht die Simulation stark vom analytischen Ergebnis ab, die Transformation ins Hauptachsensystem gewinnt in Titan also an Bedeutung. Die Korrelation zwischen $V_{z z}$ und $\eta$ erscheint im Czjzek-Diagramm als gebogene Linie. 

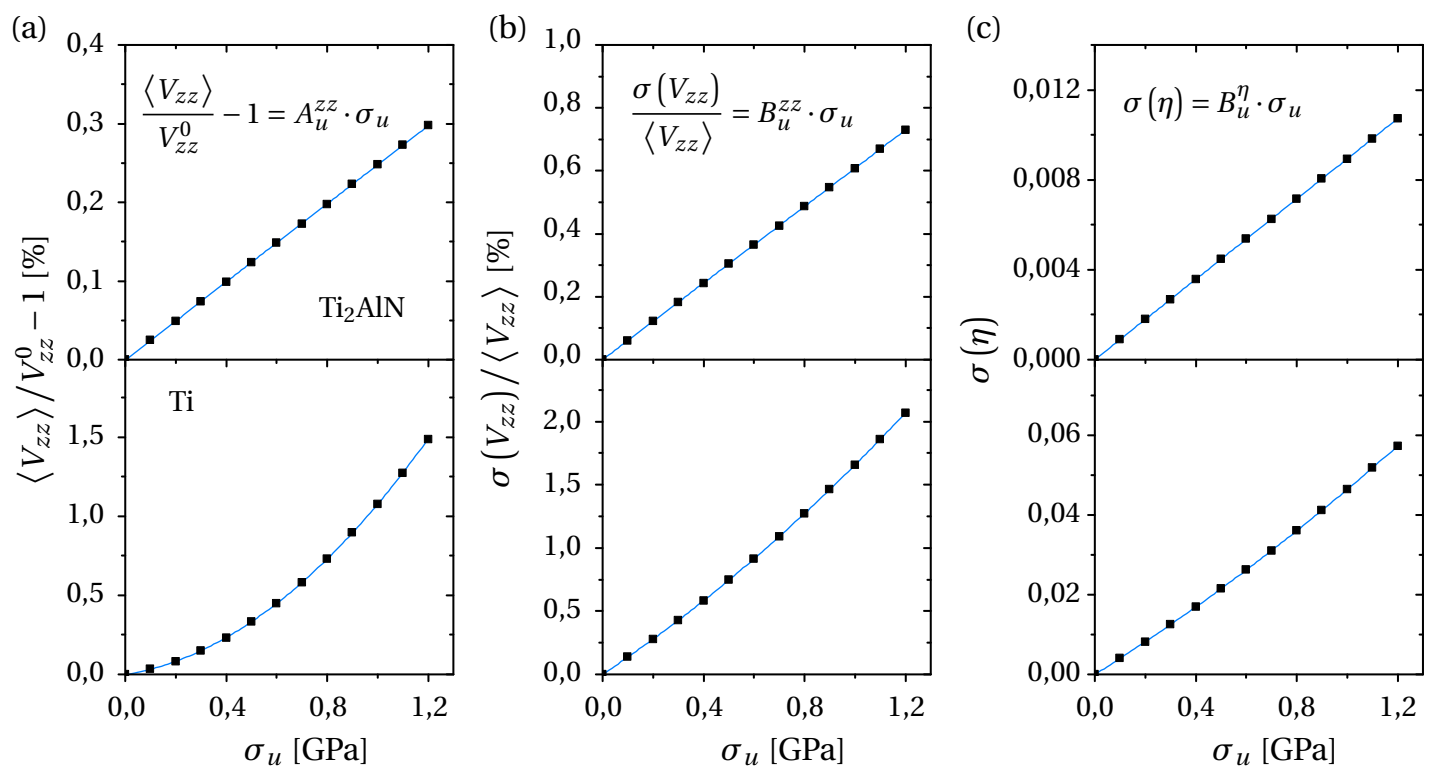

Abbildung 4.13: Relative Änderung des Mittelwerts von $V_{z z}$ (a), Standardabweichung $\sigma$ von $V_{z z}$ (b) sowie Standardabweichung von $\eta$ um den Mittelwert $\eta=0$ (c) in polykristallinem $\mathrm{Ti}_{2} \mathrm{AlN}$ und Titan als Funktion einer uniaxialen Spannung $\sigma_{u}$. Im Falle von $\mathrm{Ti}_{2} \mathrm{AlN}$ entsprechen die eingezeichneten Linien einer linearen Anpassung der simulierten Daten. Für Titan genügen die Daten einem Polynom zweiter Ordnung. Die Stärke der Nichtlinearität wird im betrachteten Spannungsbereich im Wesentlichen durch das Verhältnis zwischen ursprünglicher Stärke des EFGs $V_{z z}^{0}$ und den Gradientelastischen Konstanten $C_{44}$ und $C_{66}$ bestimmt.

bestimmt werden. Der zur Berechnung eingesetzte Winkel $\theta \in[0, \pi]$ trägt entsprechend seiner Wahrscheinlichkeitsdichte $p(\theta)=1 / 2 \sin \theta$ zur Verteilung bei (siehe oben).

Die so erhaltenen Verteilungen für $V_{z z}$ und $\eta$ sind in Abbildung 4.11 für $\mathrm{Ti}_{2} \mathrm{AlN}$ gezeigt. Simulation und analytisches Ergebnis sind nahezu identisch, die Transformation ins Hauptachsensystem spielt hier also lediglich eine untergeordnete Rolle. Anhand der Darstellung im Czjzek-Diagramm wird die Korrelation zwischen $V_{z z}$ und $\eta$ in Form einer Geraden deutlich.

Die gleiche Analyse in Titan (siehe Abbildung 4.12) zeigt, dass Simulation und analytisches Ergebnis erheblich andere Verteilungen vorhersagen. In diesem Falle gewinnt die Transformation ins Hauptachsensystem also an Bedeutung. Die Korrelation zwischen $V_{z z}$ und $\eta$ verändert sich von einer Geraden hin zu einer gebogenen Linie im CzjzekDiagramm; die Endpunkte des Pfades bleiben fixiert, da diese Punkte den reinen Normalspannungen entsprechen, in denen das Hauptachsensystem des Feldgradienten erhalten bleibt. Der Träger der beiden Verteilungen ist nach wie vor beschränkt, allerdings kann die Verteilung bei genügend hohen Spannungen über die durch reine Normalspannungen vorgegebenen Grenzen hinaus reichen.

Die Frage, ob für ein bestimmtes Materialsystem die analytische Näherung verwendet werden kann oder ob die Transformation ins Hauptachsensystem berücksichtigt werden muss, lässt sich auf den betrachteten Spannungsbereich und auf das Verhältnis zwi- 
Tabelle 4.4: Kopplungskonstanten $A_{u}^{z z}, B_{u}^{z z}$ und $B_{u}^{\eta}$, die in einem polykristallinen Material die relative Änderung des Mittelwertes von $V_{z z}$ sowie die (relative) Verbreiterung von $V_{z z}$ und $\eta$ an die uniaxiale Kompression $\sigma_{u}$ koppeln.

\begin{tabular}{cccc}
\hline Wirtsgitter & $A_{u}^{z z}\left[10^{-3} \mathrm{GPa}^{-1}\right]$ & $B_{u}^{z z}\left[10^{-3} \mathrm{GPa}^{-1}\right]$ & $B_{u}^{\eta}\left[10^{-3} \mathrm{GPa}^{-1}\right]$ \\
\hline $\mathrm{Nb}_{2} \mathrm{AlC}$ & $2,0(4)$ & $6,7(2)$ & $9,0(3)$ \\
$\mathrm{Ti}_{2} \mathrm{AlC}$ & $2,5(5)$ & $4,9(3)$ & $2,4(2)$ \\
$\mathrm{Ti}_{2} \mathrm{AlN}$ & $2,5(1)$ & $6,1(1)$ & $8,9(1)$ \\
$\mathrm{Ti}_{2} \mathrm{InC}$ & $4,9(2)$ & $7,1(1)$ & $7,0(1)$ \\
\hline
\end{tabular}

schen intrinsischem EFG und Scherkomponenten $C_{44}$ bzw. $C_{66}$ des Gradient-elastischen Tensors zurückführen. Im gegebenen Beispiel von $\mathrm{Ti}_{2} \mathrm{AlN}$ und Titan unterscheidet sich dieses Verhältnis um über eine Größenordnung. Folglich müsste die Transformation des Hauptachsensystems in $\mathrm{Ti}_{2} \mathrm{AlN}$ erst für deutlich höhere Spannungen berücksichtigt werden. Beim Übergang hin zu verschwindenden $V_{i j}^{0}$, also zu kubischen Systemen, dominiert diese Transformation den EFG-Tensor maximal. Die analytische Lösung aus Gleichung 4.11 verliert vollständig ihrer Bedeutung. Gleichzeitig wird aufgrund der fehlenden Vorzugsrichtung auch die eindeutige Korrelation zwischen $V_{z z}$ und $\eta$ aufgehoben.

Die vorherigen Aussagen, Mittelwerte und Standardabweichungen würden linear mit der Spannung ansteigen, lassen sich nun unter Berücksichtigung der Transformation ins Hauptachsensystem überprüfen. Entsprechende Darstellungen als Funktion der Spannung finden sich für $\mathrm{Ti}_{2} \mathrm{AlN}$ und Titan in Abbildung 4.13. Kann für $\mathrm{Ti}_{2} \mathrm{AlN}$ im gewählten Spannungsbereich aufgrund der Ähnlichkeit zwischen simulierter und analytischer Verteilung noch sehr wohl ein linearer Zusammenhang angenommen werden, so muss in Titan ein Polynom zweiter Ordnung herangezogen werden, um die Daten zu beschreiben. Dies resultiert, wie oben schon angedeutet, aus dem nichtlinearen Einfluss der Scherkomponenten auf die Eigenwerte des EFG-Tensors. Für die hier untersuchten MAX-Phasen genügt der lineare Zusammenhang, da die experimentellen Spannungen $1 \mathrm{GPa}$ nicht wesentlich übersteigen. Die entsprechenden Proportionalitätskonstanten $A_{u}^{z z}, B_{u}^{z z}$ und $B_{u}^{\eta}$ aus den Gleichungen 4.14, 4.15 und 4.17 sind in Tabelle 4.4 für die hier untersuchten MAX-Phasen aufgeführt.

\subsubsection{Faltungsverhalten und experimenteller Zugang}

Mit der Kenntnis über individuelle Feldgradientenverteilungen können nun Aussagen über die tatsächlich in einer Probe vorgefundene Verteilung getroffen werden. Die beobachtete Verteilung setzt sich hierbei aus der Faltung aller oben erläuterten Teilbeiträge zusammen. In diesem Zusammenhang lohnt es sich, die Auswirkungen dieser Verteilung auf das PACSpektrum zu betrachten, da bei dessen Auswertung eine Modellverteilung zugrunde gelegt werden muss.

In unbelasteten Proben ist davon auszugehen, dass sich die Feldgradientenverteilung im Wesentlichen aus Beiträgen der Punkt- und Liniendefekte zusammensetzt. Die resultierende Verteilung von $V_{z z}$ entspräche in diesen Fällen also einer Faltung aus Lorentz- 
und Gauß-Verteilung ${ }^{a}$, das heißt einem Voigt-Profil. Das zugehörige Faltungsintegral lässt sich zwar nicht im Frequenzraum analytisch lösen, jedoch aber im Zeitraum - dem Raum, in dem die Störfunktion gemessen wird. Die Faltung zerfällt dort in ein Produkt aus exponentieller und gaußförmiger Dämpfung der Störfunktion, lässt sich also als Erweiterung von Gleichung 3.11 auffassen.

Eine exemplarische Anpassung repräsentativer Messdaten in $\mathrm{Ti}_{2} \mathrm{AlN}$ mit einer auf diese Weise erweiterten Störfunktion ergibt unglücklicherweise keine eindeutigen Ergebnisse für die beteiligten Verteilungsbreiten: Unterschiedliche Messspektren der gleichen Probe zeigen stark voneinander abweichende Anteile der Verbreiterung; bei gegebener Messstatistik lassen sich diese beiden Parameter also nicht entkoppeln. Da zudem auch dieser Ansatz die Verteilung von $\eta$ ignoriert (siehe Kapitel 3.1.4), kann im Hinblick auf die Eindeutigkeit des Fits die soeben vorgeschlagene Erweiterung direkt wieder verworfen werden - ein Fit nach Gleichung 3.11 liefert bei fixiertem Parameter $a$ verlässlichere Werte für die Defektverbreiterung $\delta$. Unklar ist dabei lediglich, welcher Parameter $a$ für die Form der Dämpfung sinnvollerweise gewählt werden sollte.

Der Beitrag der Verteilung von $\eta$ zur Störfunktion lässt sich analytisch nicht beschreiben, allerdings steht $a$ priori fest, dass sich die Form der beobachteten Dämpfung über die Verteilungen von $V_{z z}$ hinaus verändert. Diese potentielle Änderung aller beteiligten Dämpfungsformen resultiert in Kombination mit dem zentralen Grenzwertsatz in der Annahme, dass sich die Dämpfung schließlich einer Gauß-Verteilung annähert, der Parameter $a=2$ also einen sinnvollen Kompromiss zwischen Eindeutigkeit und Realitätsnähe des Fits bei möglichst geringer Zahl der Freiheitsgrade bildet. Folglich reduziert sich die Frage nach dem exakten Einfluss einer Störung auf die Störfunktion in erster Ordnung auf die Untersuchung des Einflusses dieser Störung auf die effektive Dämpfung $\delta$, der Standardabweichung der Frequenzverteilung des Spektrums. Die Verteilung von $\eta$ reduziert sich dagegen in erster Ordnung auf ihren Beitrag zur Verbreiterung der Wechselwirkungsfrequenz $\omega_{n}$.

Eine solche Abschätzung am Beispiel von $\mathrm{Ti}_{2} \mathrm{AlN}$ zeigt, dass bei einer typischen Standardabweichung $\sigma\left(V_{z z}\right)$ von ungefähr $1 \%$ eine Standardabweichung $\sigma(\eta)$ von ungefähr 0,02 auftritt (siehe Abbildungen 4.9 oder 4.13), die in einer Änderung der Wechselwirkungsfrequenz $\omega_{1}$ von bis zu 0,05\% mündet (siehe Abbildung 3.2). Der Anteil der $\eta$-Verteilung an der gesamten Dämpfung beträgt in diesem Beispiel also maximal 5\%, wächst allerdings aufgrund der $\eta^{2}$-Abhängigkeit von Gleichung 3.9 für größere Verteilungsbreiten linear an. Für geringe Breiten lässt sich somit die $\eta$-Verteilung vernachlässigen. Dies gilt insbesondere für alle hier untersuchten reinen MAX-Phasen, deren Verteilungsbreiten um 1\% liegen.

Tritt neben der Defektverbreiterung auch eine elastische Verbreiterung auf, ändert sich an der bisherigen Situation wenig, lediglich eine weitere Verteilung trägt zur Verbreiterung bei. Der augenscheinlichste Unterschied besteht in der starken Asymmetrie der Verteilung von $V_{z z}$, die sich schließlich auch in der Faltung mit der Defektverbreiterung widerspiegelt. Wird die anfängliche Defektverbreiterung wie oben gezeigt als Gauß-Verteilung mit Breite $\delta_{0}$ approximiert, ergibt sich eine beobachtete Verteilung wie sie in Abbildung 4.14 dargestellt ist. Der Mittelwert dieser Verteilung wird einzig durch die Verteilung der elastischen

\footnotetext{
${ }^{a}$ Der Beitrag der Schraubenversetzungen zur Verbreiterung von $V_{z z}$ beträgt nur ein Hundertstel des Beitrages der Stufenversetzungen.
} 
(a)

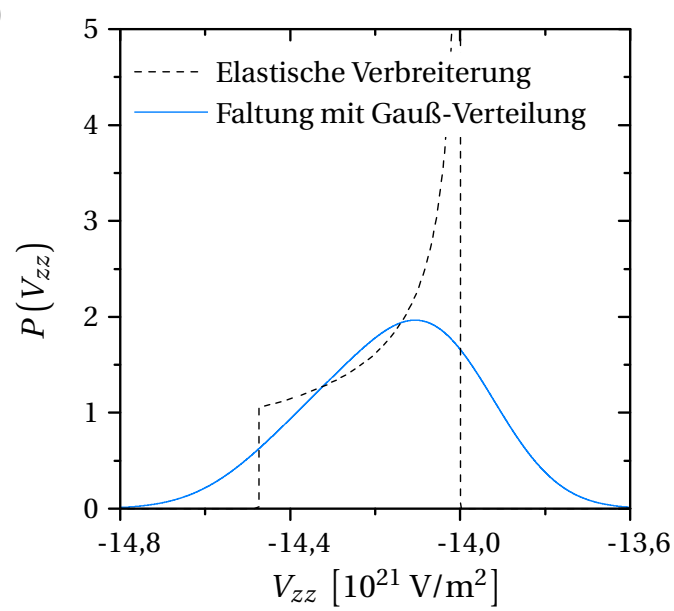

(b)

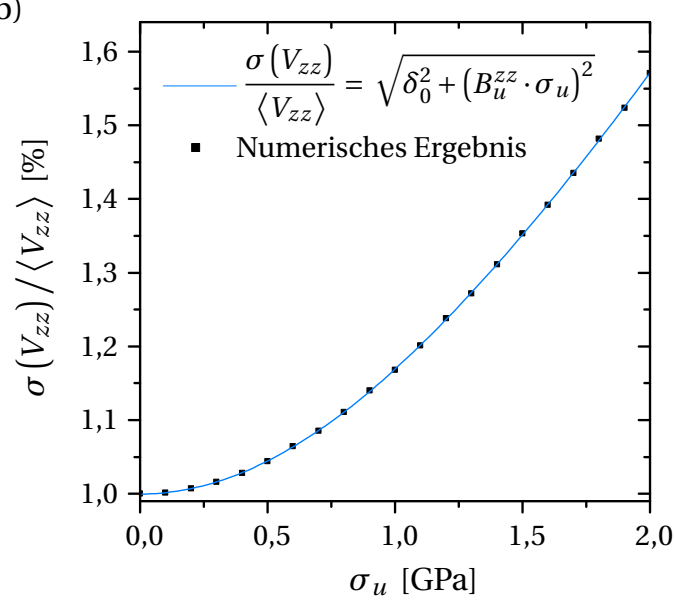

Abbildung 4.14: Faltung der Wahrscheinlichkeitsdichtefunktion von $V_{z z}$ an einem Cd-Kern in polykristallinem $\mathrm{Ti}_{2} \mathrm{AlN}$ unter uniaxialer Spannung $\sigma_{u}=1,64 \mathrm{GPa}$ mit einer GaußVerteilung der Breite $\delta_{0}$ (a). Die Standardabweichung beider Verteilungen betragen vor der Faltung jeweils $1 \%$ des Mittelwertes von $V_{z z}$, die daraus resultierende Verteilung kann ausschließlich numerisch berechnet werden und weist eine Standardabweichung von 1,41\% auf. Die Verallgemeinerung auf beliebige Spannungen zeigt, dass sich die Varianz aus der Quadratsumme der beteiligten Standardabweichungen ermitteln lässt (b).

Verbreiterung bestimmt, da die Gauß-Verteilung eine gerade Funktion ist. Eine analytische Beschreibung dieses Faltungsintegrals ist weder im Frequenz- noch im Zeitraum möglich, die numerische Berechnung zeigt aber, dass sich die Varianz der neuen Verteilung aus der Quadratsumme der Standardabweichungen der Defekt- und der elastischen Verbreiterung ergeben (siehe Abbildung 4.14b). Dieses Verhalten zeigt sich unabhängig von der Wahl der Gauß-Verteilung als glättende Funktion auch für die Faltung mit einer Lorentz-Verteilung. Die beobachtete Standardabweichung bei uniaxialer Spannung $\sigma_{u}$ und gegebener Defektverbreiterung $\delta_{0}$ ergibt sich mit Gleichung $4.15 \mathrm{zu}$

$$
\frac{\sigma\left(V_{z z}\right)}{\left\langle V_{z z}\right\rangle}=\sqrt{\delta_{0}^{2}+\left(B_{u}^{z z} \cdot \sigma_{u}\right)^{2}} .
$$

Dieser Zusammenhang bildet gemeinsam mit der vorhergehenden Analyse der Feldgradientenverteilung die Basis für die Auswertung der im nächsten Kapitel folgenden experimentellen Arbeiten.

Für eine elastische Verbreiterung in der Größenordnung der Defektverbreiterung lässt sich die Verteilung und somit auch die Dämpfung wiederum gut mit einer Gauß-Verteilung annähern; somit kann auch in diesen Fällen die Störfunktion gemäß Gleichung 3.11 angepasst werden. Der speziell bei dieser Näherung auftretende systematische Fehler liegt für alle untersuchten Materialsysteme und Messbereiche bei unter 10\% für die Bestimmung des Mittelwertes und bei unter 5\% für die Standardabweichung von $V_{z z}$. Beide Fehler lassen sich beim Vergleich mit experimentellen Werten einkalkulieren. 


\section{Kapitel 5}

\section{Experimentelle Ergebnisse}

Auf die theoretische Vorarbeiten folgend, werden in diesem Kapitel drei verschiedene Experimente zu unterschiedlichen Formen der Unordnung in MAX-Phasen vorgestellt. Als experimentelle Ausgangslage aller drei Experimente können die Vorarbeiten von Jürgens [47] betrachtet werden, in denen der elektrische Feldgradient an der Cadmiumsonde in diversen reinen MAX-Phasen systematisch untersucht wurde. Im ersten Experiment wird der Einfluss einer uniaxialen Spannung auf die Feldgradientenverteilung reiner MAX-Phasen im Hinblick auf elastische Verbreiterung und Defektverbreiterung studiert. Beim zweiten Experiment werden - im Kontrast zu den sonst untersuchten Volumenproben - Dünnschichtproben der MAX-Phasen auf eine etwaige durch interne Spannungen hervorgerufene Feldgradientenverbreiterung hin untersucht. Das zuletzt vorgestellte Experiment befasst sich mit einer Form der Defektverbreiterung, die kontinuumstheoretisch nicht erfassbar ist und im ersten Experiment nicht zu Tage tritt: Die Verbreiterung in Substitutionsmischkristallen der zuvor noch reinen MAX-Phasen. Die beobachteten Effekte lassen sich somit einerseits langreichweitigen Einflüssen der Dehnungsfelder und andererseits kurzreichweitigen Auswirkungen lokalen Umgebung zuordnen. An geeigneter Stelle wird wie schon in Kapitel 4 auf vergleichende Experimente an Titan oder Zink zurückgegriffen.

Eine vor allen Experimenten durchgeführte Röntgenstrukturanalyse der untersuchten Proben ermöglicht es, die Phasenreinheit zu beurteilen. Die Auswertung der zugehörigen Diffraktogramme mit der Rietveld-Methode ergibt die in Tabelle 5.1 gezeigten strukturellen Parameter der untersuchten Phasen. Der Vergleich zum jeweiligen Literaturwert zeigt insbesondere auch bei der Vergleichsmessung an Lanthanhexaborid-Pulver (NIST-Standard 660B [181]) nur geringe Abweichungen. Die in manchen MAX-Phasen enthaltenen Verunreinigungen beschränken sich im Allgemeinen auf Metallcarbide mit Massenanteilen kleiner 3\%; lediglich in $\mathrm{Nb}_{2} \mathrm{AlC}$ liegt der Anteil an Niobcarbid - je nach Probe - bei über $10 \%$. Aus der Rietveld-Anpassung geht hervor, dass die meisten Proben eine zum Teil starke Textur aufweisen. Dies wird anhand des Unterschieds zwischen den gemessenen und den bei regelloser Orientierung erwarteten Intensitäten deutlich. Die hierbei einfließende Vorzugsrichtung der Körner erlaubt es zwar den Fit zu verbessern, genügt aber nicht zur vollständigen Bestimmung der Orientierungsdichteverteilungsfunktion. Ein exemplarisches Diffraktogramm samt Anpassung findet sich in Unterkapitel 5.3 neben der Beschreibung der neu entdeckten MAX-Phase $\mathrm{Ti}_{2}\left(\mathrm{Al}_{0,5}, \mathrm{In}_{0,5}\right) \mathrm{C}$.

Alle PAC-Messungen nutzen per Ionenimplantation eingebrachte ${ }^{111} \mathrm{In} /{ }^{111} \mathrm{Cd}$-Sonden zur Messung des elektrischen Feldgradienten (siehe Kapitel 3). Die Sonden befinden sich, falls nicht anders beschrieben, nach fünfstündigem Ausheilen bei $1173 \mathrm{~K}$ zum größten Teil 
Kapitel 5 Experimentelle Ergebnisse

Tabelle 5.1: Übersicht der experimentell ermittelten Gitterkonstanten aller untersuchten Materialien. Zum Vergleich sind, falls bekannt, die entsprechenden Literaturwerte angegeben.

\begin{tabular}{ccccc}
\hline & $a[\AA]$ & $a_{\text {lit }}[\AA]$ & $c[\AA]$ & $c_{\text {lit }}[\AA]$ \\
\hline $\mathrm{Cr}_{2} \mathrm{AlC}$ & $2,862(5)$ & $2,860[4]$ & $12,81(2)$ & $12,82[4]$ \\
$\mathrm{Nb}_{2} \mathrm{AlC}$ & $3,108(1)$ & $3,103[4]$ & $13,888(3)$ & $13,83[4]$ \\
$\mathrm{Ti}_{2} \mathrm{AlC}$ & $3,061(2)$ & $3,04[4]$ & $13,66(1)$ & $13,60[4]$ \\
$\mathrm{Ti}_{2} \mathrm{AlN}$ & $2,988(2)$ & $2,994[5]$ & $13,62(1)$ & $13,61[5]$ \\
$\mathrm{Ti}_{2} \mathrm{InC}$ & $3,133(5)$ & $3,132[6]$ & $14,08(1)$ & $14,06[6]$ \\
$\left(\mathrm{Ti}_{0,5}, \mathrm{Nb}_{0,5}\right)_{2} \mathrm{AlC}$ & $3,077(1)$ & $3,077(1)[34]$ & $13,795(8)$ & $13,790(1)[34]$ \\
$\left(\mathrm{Ti}_{0,5}, \mathrm{~V}_{0,5}\right)_{2} \mathrm{AlC}$ & $2,979(1)$ & $2,975[35]$ & $13,396(5)$ & $13,393[35]$ \\
$\mathrm{Ti}_{2}\left(\mathrm{Al}_{0,5}, \mathrm{In}_{0,5}\right) \mathrm{C}$ & $3,093(1)$ & - & $13,871(2)$ & - \\
$\mathrm{Ti}_{2} \mathrm{Al}_{(}\left(\mathrm{C}_{0,5}, \mathrm{~N}_{0,5}\right)$ & $3,020(1)$ & $3,021[36]$ & $13,626(2)$ & $13,61[36]$ \\
$\mathrm{Ti}$ & $2,953(1)$ & $2,9511[193]$ & $4,689(1)$ & $4,6843[193]$ \\
$\mathrm{Zn}$ & $2,664(1)$ & $2,664(1)[193]$ & $4,95(2)$ & $4,944(1)[193]$ \\
$\mathrm{LaB}$ & $4,155(1)$ & $4,15691(8)[181]$ & - & - \\
\hline
\end{tabular}

auf dem A-Platz der MAX-Phasen [47], nach einstündigem Ausheilen bei $573 \mathrm{~K}$ auf dem ZnPlatz in Zink [188] bzw. nach fünfminütigem Ausheilen bei $473 \mathrm{~K}$ auf dem Ti-Platz in Titan. Die Anpassung der gemessenen Störfunktion erfolgt stets mit Gleichung 3.11 unter der Annahme einer gaußförmigen Dämpfung und variablen Amplituden $s_{2 n}$. Letzteres trägt der vorhanden Textur Rechnung, ändert sich also unmittelbar mit einer Drehung der Probe in der Detektorebene. Da keine systematische Änderung dieser Amplituden während der hier durchgeführten Experimente beobachtet wird, beschränken sich die folgenden Untersuchungen auf die Quadrupolfrequenz $\omega_{Q}$, den Asymmetrieparameter $\eta$ und die Dämpfung $\delta$. Dazu abgegrenzt steht der im vorherigen Kapitel theoretisch bestimmte mittlere Feldgradient $V_{z z}$ und der Asymmetrieparameter $\eta$ bzw. deren Verteilungsbreiten $\sigma\left(V_{z z}\right)$ und $\sigma(\eta)$. Quadrupolfrequenz und Feldgradient sind über das Quadrupolmoment $Q$ verbunden, die Verteilungsbreiten wirken sich auf die Dämpfung der Störfunktion aus (siehe Kapitel 4.2.5). Um die experimentellen Ergebnisse mit den theoretischen Erwartungen vergleichen zu können, werden Feldgradient und Standardabweichungen in Quadrupolfrequenz und Dämpfung umgerechnet. Der Asymmetrieparameter $\eta$ wird separat anhand der Kreuzkorrelation im Czjzek-Diagramm diskutiert.

Sowohl die Amplituden $s_{2 n}$ der PAC-Spektren als auch die Intensitäten der Röntgendiffraktogramme werden, wie oben angedeutet, durch die Vorzugsrichtung der implantierten bzw. im Wechselwirkungsbereich liegenden Kristallite beeinflusst. Da dieser Messbereich nur eine beschränke Anzahl an Körnern umfasst, tragen neben der globalen, durch den Herstellungsprozess bedingten Orientierungsdichte auch statistische Effekte zur Orientierung der Messspektren bei. Dieser Aspekt ist insbesondere für Zink relevant, da hier die Korngröße mit einigen hundert Mikrometern um eine Größenordnung über der der anderen Proben liegt. Wenngleich die Orientierung der Spektren nicht weiter quantifiziert wird, muss sie doch bei der Auswertung, insbesondere bei der elastischen Verbreiterung, stets bedacht werden. 


\subsection{Uniaxiale Kompression ${ }^{a}$}

Durch eine uniaxiale Kompression der MAX-Phasen können simultan der Einfluss der elastischen Verbreiterung auf das PAC-Spektrum sowie die durch die Last hervorgerufene irreversible Verformung studiert werden. Eine Messreihe besteht aus mehreren Belastungszyklen, bei denen jeweils eine bestimmte Spannung zunächst angelegt und anschließend wieder weggenommen wird. Sowohl im belasteten als auch im unbelasteten Zustand eines Zyklus wird eine PAC-Messung durchgeführt. Die Proben liegen dabei immer flach in der Detektorebene, die Last wirkt senkrecht auf diese Ebene. Die dabei auftretende uniaxiale Belastung $\sigma_{u}$ ergibt sich näherungsweise aus dem Verhältnis zwischen Kraft und Querschnittsfläche der Probe nach dem jeweiligen Belastungszyklus. Eine während der Belastung auftretende elastische Vergrößerung der Querschnittsfläche wird hierbei vernachlässigt. Im Gegensatz zu $\sigma_{u}$ ergibt sich die technische Spannung $\sigma_{e}$ aus Kraft und Querschnittsfläche vor der Deformation. Beide Größen lassen sich über die irreversible Deformation $\varepsilon_{p}$ gemäß $\sigma_{t}=\sigma_{e}\left(1-\varepsilon_{p}\right)$ ineinander umrechnen. Kompressive Spannung und Dehnung sind in diesem Zusammenhang positiv definiert. Die Unterscheidung zwischen reversiblen oder irreversiblen Einflüssen auf die Störfunktion erfolgt durch einen Vergleich der Messungen im belasteten und unbelasteten Zustand.

Auf diese Weise werden die MAX-Phasen $\mathrm{Nb}_{2} \mathrm{AlC}, \mathrm{Ti}_{2} \mathrm{AlC}, \mathrm{Ti}_{2} \mathrm{AlN}$ und $\mathrm{Ti}_{2} \mathrm{InC}$ sowie die hexagonalen Systeme Titan und Zink untersucht. Die gemessenen Störfunktionen lassen sich in allen Systemen bis auf $\mathrm{Ti}_{2} \mathrm{InC}$ mit einem einzigen Anteil $f_{i}$ einer von null verschiedenen Quadrupolfrequenz anpassen; nahezu alle Sonden sitzen auf dem A-Platz. Eine Übersicht über die zugehörigen Quadrupolkopplungskonstanten $v_{O}^{0}$ und Dämpfungen $\delta_{0}$ findet sich im hinteren Teil dieses Kapitels in Tabelle 5.2. In $\mathrm{Ti}_{2} \mathrm{InC}$ tritt ein zweiter Anteil mit einer Quadrupolkopplungskonstante von 17, 1(4) MHz auf, der metallischem Indium zuzuordnen ist $[47,195]$ und im Folgenden unberücksichtigt bleibt. Alle so beobachteten Feldgradienten weisen im Czjzek-Diagramm ein Maximum bei $\eta=0$ auf, sind also entsprechend der Symmetrie $D_{3 h}$ des Gitterplatzes axialsymmetrisch. Die Dämpfung der experimentellen Störfunktionen liegt im Bereich von 1\%, lediglich in Titan wird eine Dämpfung von 4,9(2)\% beobachtet, die vermutlich auf Verunreinigungen durch Sauerstoff oder auf übrig bleibende Implantationsschäden zurückzuführen ist. Die große Verteilungsbreite des Feldgradienten in Titan führt beim Fit nach Gleichung $3.11 \mathrm{zu}$ einem scheinbar von null verschiedenen Asymmetrieparameter von 0,13(3). Dieses Artefakt ist auf die beschränkte Gültigkeit von Gleichung 3.11 zurückzuführen; auch in Titan liegt ein eigentlich axialsymmetrischer EFG vor (vgl. Kapitel 3.1.4).

\subsubsection{Qualitative Beschreibung}

Das beobachtete Deformationsverhalten unterscheidet sich zwischen den Materialsystemen und wird maßgeblich durch die flache Probengeometrie geprägt. Während sich Titan ab dem Erreichen der Streckgrenze über den gesamten Messbereich plastisch verformt, verhalten sich Zink und insbesondere die MAX-Phasen nur sehr begrenzt duktil. Durch die flache Geometrie lassen sich jedoch selbst dann noch PAC-Spektren aufnehmen, wenn

${ }^{a}$ Dieses Kapitel basiert im Wesentlichen auf Ergebnissen, die in Referenz [194] veröffentlicht wurden. 
(a)

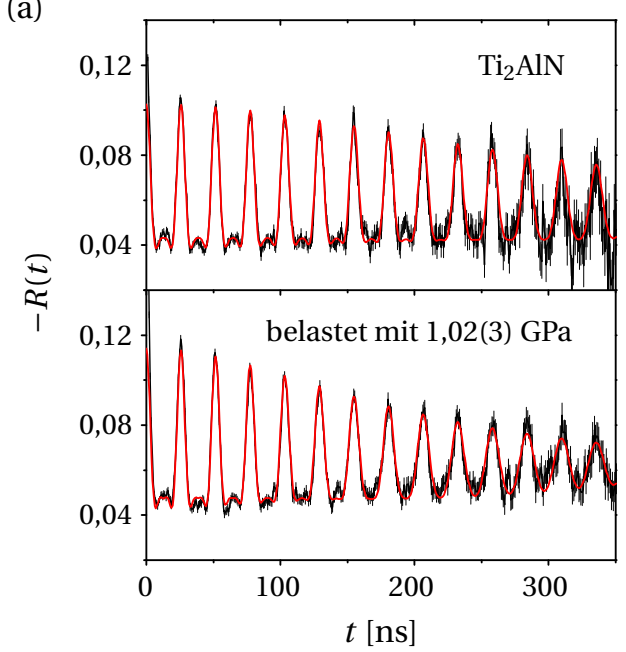

(b)

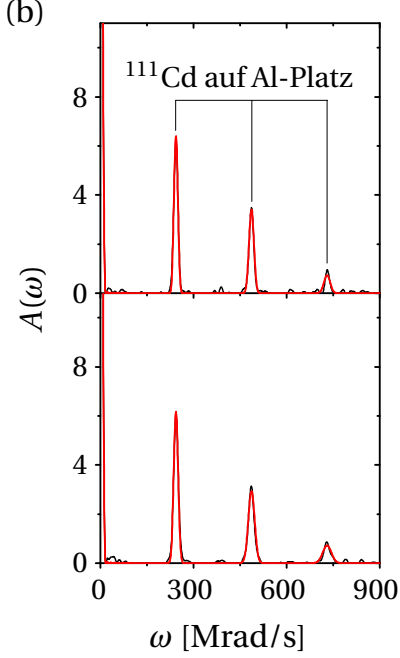

(c)
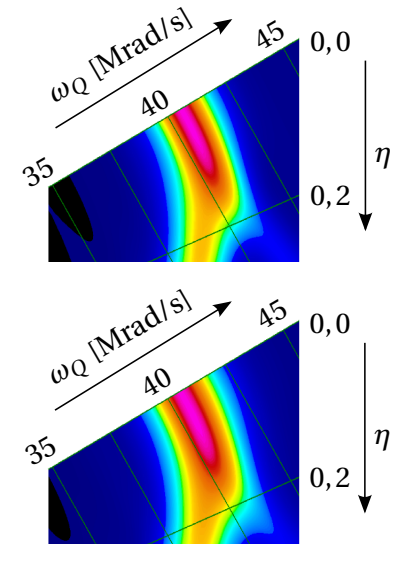

Abbildung 5.1: Exemplarische Darstellung des Einflusses einer uniaxialen Spannung auf die Störfunktion $R(t)$ (a), deren Fouriertransformierten $A(\omega)$ (b) und dem zugehörigen Czjzek-Diagramm (c) an der Cd-Sonde in $\mathrm{Ti}_{2} \mathrm{AlN}$. Die schwarze Linie entspricht den Messdaten, die rote Linien einem Fit. Der Vergleich zwischen unbelasteter Probe (oben) und belasteter Probe (unten) zeigt, dass die Dämpfung und somit die Feldgradientenverteilung, unter Last zunimmt. Im Czjzek-Diagramm wird dies durch eine Verbreiterung sowohl von $\omega_{Q}$ als auch von $\eta$ sichtbar. Die Verschiebung von $\omega_{Q}$ lässt sich aufgrund der geringen Änderung nicht erkennen.

die Druckfestigkeit der Probe schon überschritten ist. Die nachfolgend angegebenen Werte der irreversiblen Verformung $\varepsilon_{p}$ sollten deswegen nicht als plastische Dehnung fehlinterpretiert werden; sie dienen lediglich dazu, ein grobes Maß der permanenten Deformation der Probe zu vermitteln.

Ein exemplarisches PAC-Spektrum einer Probe unter Last ist in Abbildung 5.1 gezeigt. Die aufgrund der elastischen Verbreiterung erwartete Änderung der mittleren Quadrupolfrequenz fällt in diesem Beispiel so klein aus, dass sich die Periodizität der Störfunktion kaum von der unbelasteten Probe unterscheiden lässt. Gleiches gilt für die Amplituden der Übergangsfrequenzen, wenngleich hier kein Effekt erwartet wird. Die deutlichste Änderung betrifft die Dämpfung der Störfunktion, die unter Last signifikant zunimmt. Im Czjzek-Diagramm wird dies durch eine Verbreiterung der Verteilung sowohl von $\omega_{Q}$ als auch von $\eta$ sichtbar. Das Auftreten zusätzlicher Übergangsfrequenzen, wie sie bei einer Phasenseparation oder durch von der Sonde eingefangene Punktdefekte [143] entstehen würden, kann nicht beobachtet werden.

Die unter Last theoretisch vorhergesagte Korrelation zwischen $\omega_{Q}$ und $\eta$ ist nicht sichtbar. Im Czjzek-Diagramm würde diese Korrelation als schräge Linie auftauchen (vgl. Abbildung 4.11), die damit einhergehende Dynamik geht aber in der Defektverbreiterung vollständig unter. Es kommt lediglich zur besagten Verbreiterung von $\omega_{Q}$ und $\eta$. Das Maximum der Kreuzkorrelation liegt auch noch unter Last bei $\eta=0$.

Wird eine komplette, wie oben beschriebene Messreihe aufgenommen, zeigt sich, wo- 


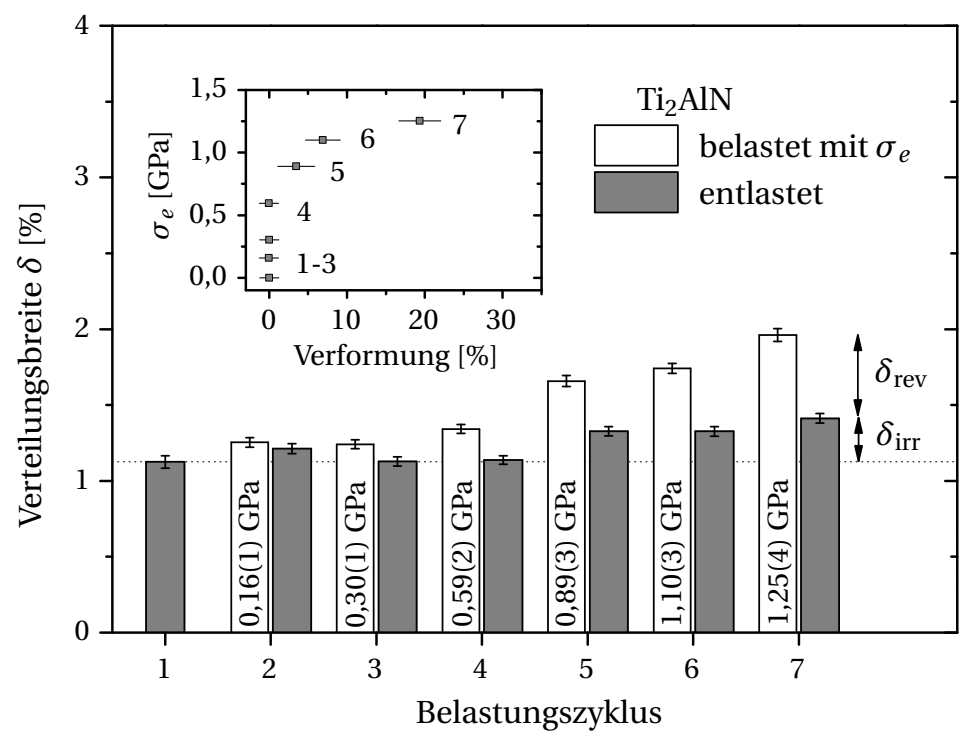

Abbildung 5.2: Verteilungsbreite $\delta$ des EFGs an der Cd-Sonde während und nach unterschiedlichen Belastungszyklen am Beispiel von $\mathrm{Ti}_{2} \mathrm{AlN}$. In einem Zyklus wird die polykristalline Probe bis zur technischen Spannung $\sigma_{e}$ uniaxial beladen und anschließend wieder entlastet. Die durch diese Belastung hervorgerufene permanente Deformation ist im eingeschobenen Graphen dargestellt und geht mit einer irreversiblen Verbreiterung $\delta_{\text {irr }}$ einher. Zusätzlich dazu, erhöht sich die Verteilungsbreite unter Last reversibel um $\delta_{\text {rev }}$. Diese elastische Verbreiterung hängt von der wahren Spannung $\sigma_{u}$ ab.

durch der Anstieg der Dämpfung hervorgerufen wird (siehe Abbildung 5.2). Die mit jedem Zyklus zunehmende technische Spannung $\sigma_{e}$ führt zu einem sukzessiven Anstieg der Dämpfung $\delta$. Wird die Last am Ende eines Zyklus weggenommen, sinkt $\delta$ auf ein niedrigeres Niveau $\mathrm{ab}^{a}$. Für die ersten Zyklen liegt dieses Niveau ungefähr bei der anfänglichen Dämpfung $\delta_{0}$; wird jedoch die Streckgrenze des Materials überschritten, verbleibt die Dämpfung auf einem intermediären Niveau. Es existiert also eine reversible Änderung $\delta_{\text {rev }}$, die sinnvollerweise von der wahren Spannung $\sigma_{u}$ abhängt, und eine irreversible Änderung $\delta_{\text {irr }}$, die mit der permanenten Deformation $\varepsilon_{p}$ und den dadurch eingebrachten Defekten verknüpft ist. Das Anlegen einer Last, die kleiner als die bisherige Maximalspannung ist, führt zu keiner weiteren signifikanten irreversiblen Änderung der Dämpfung, die reversible Änderung zeigt sich in diesem Falle nach wie vor (nicht gezeigt). Durch ein erneutes Ausheilen der Probe unter gleichen Bedingungen wie nach der Implantation lässt sich die irreversibel erhöhte Dämpfung wieder auf den anfänglichen Wert $\delta_{0}$ reduzieren (nicht gezeigt).

Die oben aufgeführten Beobachtungen treffen in ihrer Kernaussage auf alle hier untersuchten Materialsysteme zu. Individuelle Unterschiede der einzelnen Materialien zeigen sich beispielsweise in der Spannungsabhängigkeit der Quadrupolkopplungskonstante und der Dämpfung. Diese Abhängigkeiten werden im Folgenden quantitativ untersucht. Auf-

${ }^{a}$ Auch die Quadrupolfrequenz nimmt im entlasteten Zustand wieder den ursprünglichen Wert an. 
Kapitel 5 Experimentelle Ergebnisse

Tabelle 5.2: Übersicht über die Änderung der Quadrupolkopplungskonstante und der Dämpfung der Störfunktion an der Cd-Sonde mit einer uniaxialen Spannung in MAX-Phasen und ausgewählten hexagonalen Metallen. Den experimentell ermittelten sind die theoretisch erwarteten Größen bei vollständig regelloser Orientierung der Kristallite gegenüber gestellt. In beiden Fällen wurde das in Kapitel 4.2.5 beschriebene Faltungsverhalten der Dichtefunktion elastischer Verbreiterung berücksichtigt. Zum Vergleich sind Quadrupolkopplungskonstante und Dämpfung vor der Belastung mit angegeben.

\begin{tabular}{ccccccc}
\hline Wirtsgitter & $\begin{array}{c}v_{Q}^{0}[\mathrm{MHz}] \\
\text { exp. }\end{array}$ & $\begin{array}{c}\delta_{0}[\%] \\
\text { exp. }\end{array}$ & $\begin{array}{c}\mathrm{d} \ln v_{Q} / \mathrm{d} \sigma_{u}\left[10^{-3} \mathrm{GPa}^{-1}\right] \\
\text { exp. }\end{array}$ & $\begin{array}{c}\mathrm{d} \delta_{\text {rev }} / \mathrm{d} \sigma_{u}\left[10^{-3} \mathrm{GPa}^{-1}\right] \\
\text { ber. }\end{array}$ & exp. & ber. \\
\hline $\mathrm{Nb}_{2} \mathrm{AlC}$ & $243,5(3)$ & $0,9(1)$ & $-0,2(6)$ & $2,0(4)$ & $11,6(7)$ & $6,7(2)$ \\
$\mathrm{Ti}_{2} \mathrm{AlC}$ & $277,6(2)$ & $0,9(1)$ & $0,5(5)$ & $2,5(5)$ & $5,8(3)$ & $4,9(3)$ \\
$\mathrm{Ti}_{2} \mathrm{AlN}$ & $258,1(3)$ & $1,1(1)$ & $-0,9(5)$ & $2,5(1)$ & $12,0(4)$ & $6,1(1)$ \\
$\mathrm{Ti}_{2} \mathrm{InC}$ & $292,8(6)$ & $0,5(2)$ & $2,4(14)$ & $4,9(2)$ & $20(3)$ & $7,2(1)$ \\
$\mathrm{Zn}$ & $133,3(3)$ & $0,7(1)$ & $-86(6)$ & $-24(2)$ & $150(12)$ & $107(2)$ \\
$\mathrm{Ti}$ & $27,3(2)$ & $4,9(2)$ & $22(2)^{a}$ & $11(5)^{a}$ & $20(10)^{a}$ & $17(3)^{a}$ \\
\hline
\end{tabular}

${ }^{a}$ Ausgewertet bei $\sigma_{u}=1 \mathrm{GPa}$.

grund der ungenügenden Dehnungsbestimmung wird auf einer Untersuchung der Dehnungsabhängigkeit der jeweiligen Parameter verzichtet. Der Zusammenhang zu den Defektdichten wird in Kapitel 6 diskutiert.

\subsubsection{Spannungsabhängigkeit der Quadrupolkopplungskonstante}

Die Spannungsabhängigkeit der Quadrupolkopplungskonstante ist in Abbildung 5.3 für die untersuchten Materialsysteme gezeigt. Zum Vergleich sind die jeweils extremalen Änderungen einer Belastung in der bzw. senkrecht zur Basalebene sowie die mittlere Änderung des Feldgradienten, wie er bei regelloser Kornorientierung erwartet wird, mit eingezeichnet. Beide Verläufe basieren auf den Gradient-elastischen Konstanten aus Kapitel 4. Die experimentellen Werte liegen für alle Systeme innerhalb der einkristallinen Grenzen. Im Falle der MAX-Phasen ergibt sich eine gute Übereinstimmung zwischen experimenteller und theoretischer Änderung der Quadrupolfrequenz; die beobachteten Abhängigkeiten sind durchweg relativ klein. In Zink und Titan ergeben sich größere Änderungen, die eine deutliche Diskrepanz zum theoretisch erwarteten Verlauf aufweisen. Neben den Fehlern der Gradient-elastischen Konstanten führt auch die in den Proben vorhanden Textur bzw. die Vorzugsrichtung der implantierten Körner zu einer Abweichung vom regellosen Mittel.

Eine Übersicht über die gemessenen und berechneten Änderungen findet sich in Tabelle 5.2. Entsprechend der theoretischen Erwartung werden hierfür die Messdaten in den MAXPhasen und Zink mit einem linearen Fit angepasst. In Titan wird ein quadratischer Verlauf erwartet, weswegen in diesem Falle auf den linearen Fit verzichtet und stattdessen der Einfachheit halber die bei 1 GPa erwartete Änderung zum Vergleich herangezogen wird ${ }^{b}$.

\footnotetext{
${ }^{b}$ Die Form dieses Verlaufs hängt maßgeblich von den Gradient-elastischen Konstanten ab. Da aber diese Konstanten in Titan sehr ungenau bestimmt wurden (siehe Kapitel 4), ergibt ein direkter Vergleich des Verlaufs mit experimentellen Daten wenig Sinn.
} 
(a)

(c)

(e)

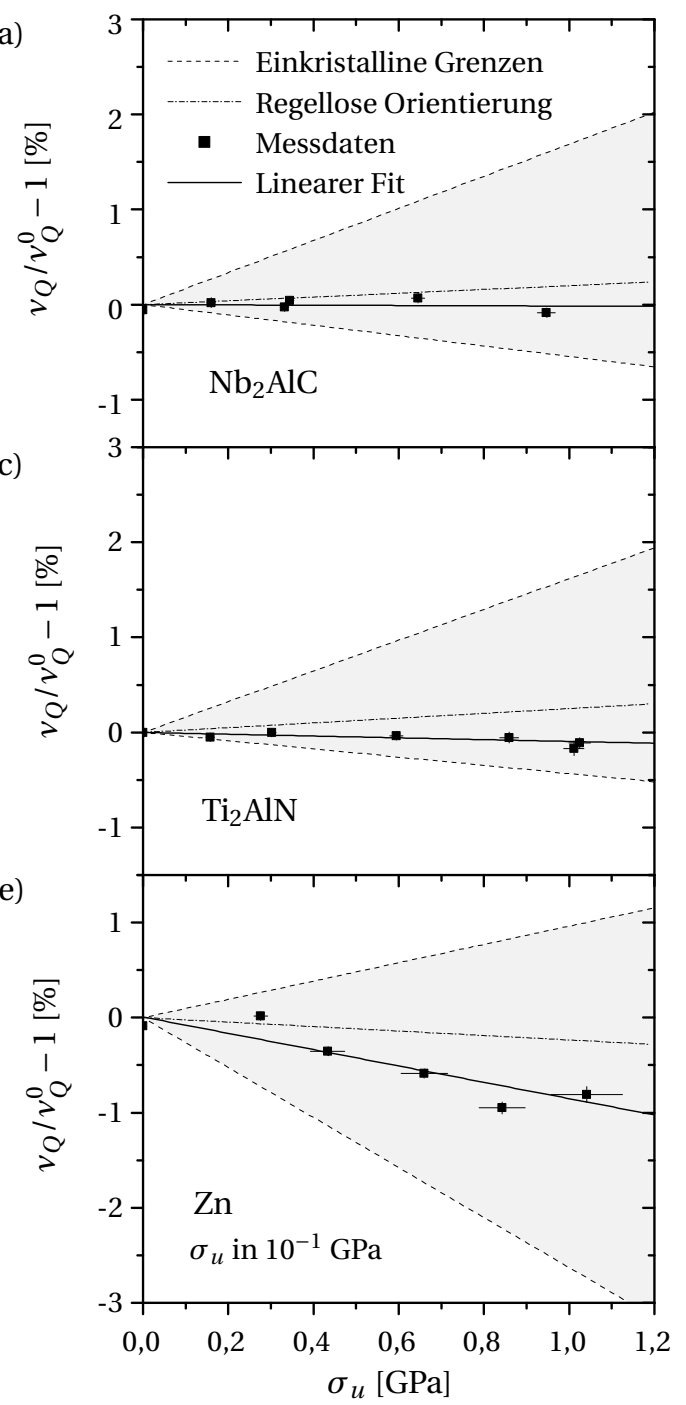

(b)

(d)

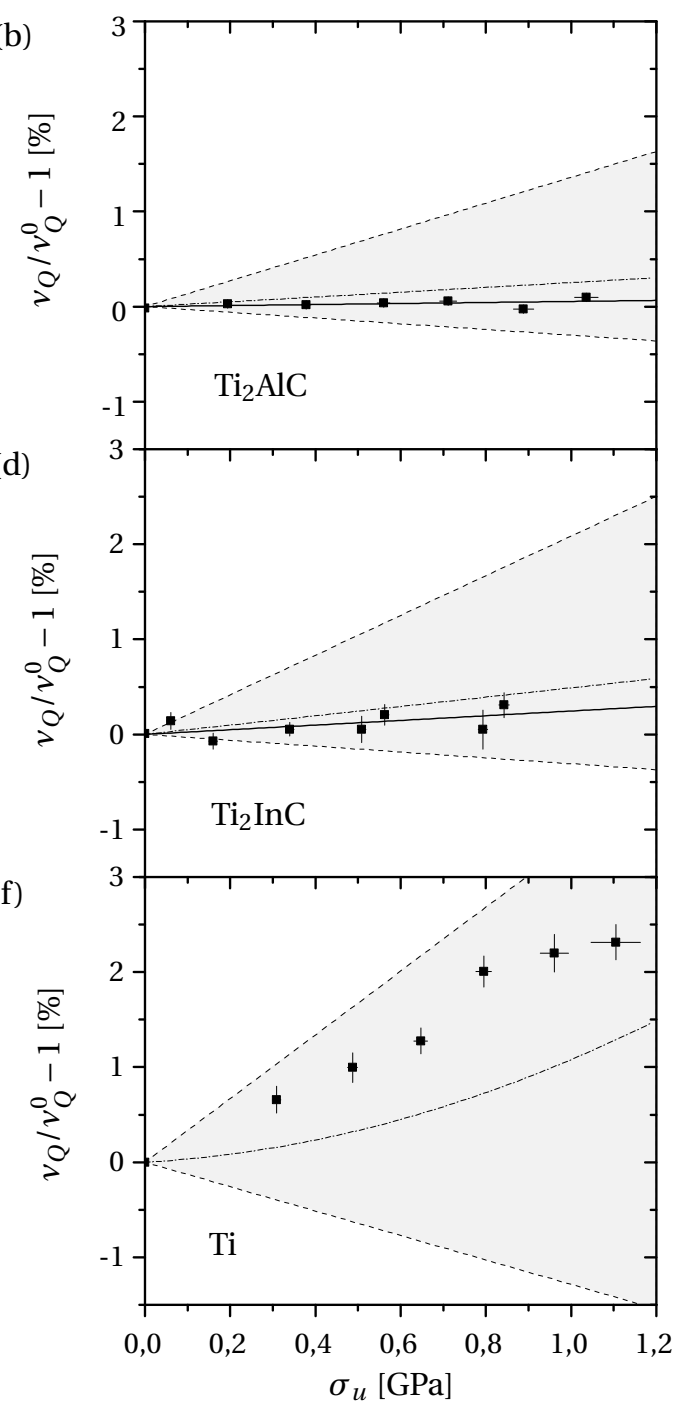

Abbildung 5.3: Experimentelle Änderung der Quadrupolkopplungskonstante $v_{Q}$ mit zunehmender uniaxialer Spannung $\sigma_{u}$ an Cd-Kernen in polykristallinen MAX-Phasen, Zn und $\mathrm{Ti}$ (a-f). Die experimentell gefundenen Änderungen liegen innerhalb der theoretischen Schranken (grau), die durch eine uniaxiale Belastung entlang der Kristallachsen gegeben sind. Die theoretisch erwarteten Änderungen für Materialien mit vollständig regelloser Orientierung zeigen teils große Abweichungen zum Experiment. Die Abweichungen lassen sich zum einen auf Unsicherheiten der entsprechenden Gradient-elastischen Konstanten, zum anderen auf eine Textur in den Proben zurückführen. Im Falle von Ti wurde auf eine lineare Anpassung der Messdaten verzichtet, da ein quadratischer Verlauf erwartet wird (f). 


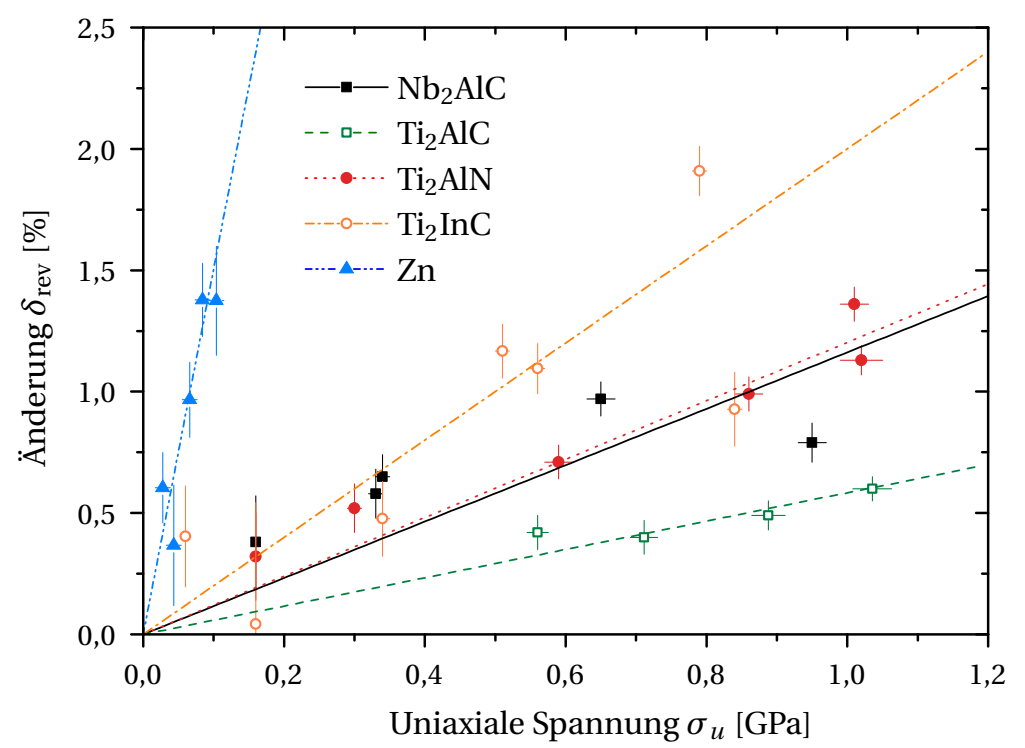

Abbildung 5.4: Reversible Änderung der Verteilungsbreite $\delta$ mit zunehmender uniaxialer Spannung $\sigma_{u}$ des EFGs an der Cd-Sonde in polykristallinen MAX-Phasen und Zn. Die Messdaten wurden unter Berücksichtigung des in Kapitel 4.2.5 beschriebenen Faltungsverhaltens der Dichtefunktion elastischer Verbreiterung ermittelt. Die eingezeichneten Linien entsprechen, gemäß der theoretischen Erwartung, einer linearen Anpassung. Auf eine Darstellung der Messdaten in Ti wurde aufgrund der großen anfänglichen EFG-Verteilungsbreite und der damit verbundenen experimentellen Unsicherheit in der Bestimmung der reversiblen Änderung mit dem Druck verzichtet; eine Abschätzung ist in Tabelle 5.2 gegeben.

\subsubsection{Spannungsabhängigkeit der Verteilungsbreite}

Die Spannungsabhängigkeit der Dämpfung ist in Abbildung 5.4 für die untersuchten Materialsysteme gezeigt. Die Bestimmung der dabei dargestellte reversiblen Änderung der Dämpfung basiert auf dem in Kapitel 4.2.5 untersuchten Faltungsverhalten der beteiligten Verteilungsfunktionen, wonach sich das Quadrat der unter Last beobachteten Dämpfung $\delta$ aus der Summe der Quadrate des reversiblen Anteils $\delta_{\text {rev }}$ und der nach Wegnahme der Last übrig bleibenden Restdämpfung ergibt. Unter diesen Voraussetzungen gilt:

$$
\delta^{2}=\delta_{0}^{2}+\delta_{\text {irr }}^{2}+\delta_{\text {rev }}^{2}
$$

Gemäß Gleichung 4.15 wird erwartet, dass sich der reversible Anteil für alle Systeme näherungsweise linear mit der Spannung ändert (vgl. Abbildung 4.13). Folglich wird eine Ausgleichsgerade zum Anpassen der Daten herangezogen

Die materialspezifischen Steigungen dieser Ausgleichsgeraden sind in Tabelle 5.2 aufgeführt und den aus den berechneten Gradient-elastischen Konstanten erwarteten Steigungen der elastischen Verbreiterung gegenüber gestellt. Hierbei übersteigt die experimentell gemessene Änderung in allen Materialsystemen die theoretische Vorhersage. Die Implikationen dieser Beobachtung werden in Kapitel 6 diskutiert. 


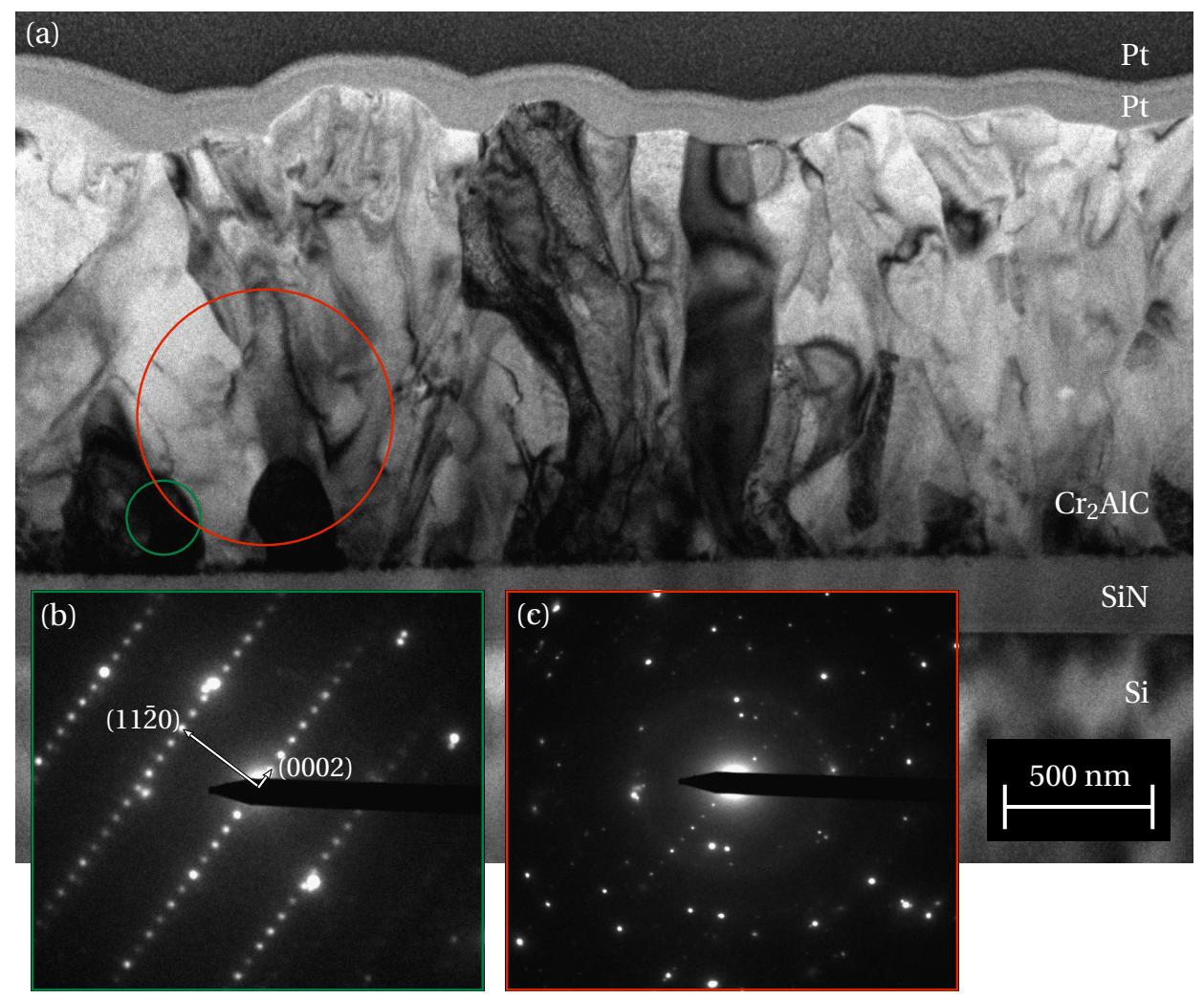

Abbildung 5.5: TEM-Aufnahme eines Querschnitts der $\mathrm{Cr}_{2} \mathrm{AlC}$-Dünnschichtprobe in Hellfeldabbildung (a). Die 1,3(1) $\mu \mathrm{m}$ dicke $\mathrm{Cr}_{2}$ AlC-Schicht wurde auf einem Siliziumeinkristall deponiert, der zuvor mit amorphem Siliziumnitrid beschichtet wurde. Die beiden Platinschichten stammen aus der TEM-Probenpräparation. Dunkelfeldabbildungen zeigen, dass sich die $\mathrm{Cr}_{2} \mathrm{AlC}$-Schicht aus länglichen Kristalliten mit einer Größe zwischen $100 \mathrm{~nm}$ und $600 \mathrm{~nm}$ zusammensetzt. Anhand der Feinbereichsbeugung eines ausgewählten Kristallits (grün) wird das hohe $c / a$-Verhältnis der MAX-Phasen deutlich (b). Wird das Beugungsbild eines größeren Bereiches (rot) betrachtet, zeigen sich Reflexe unterschiedlich orientierter Kristallite (c).

\subsection{Dünnschichtsysteme}

Bei der Untersuchung der Dünnschichtsysteme ist insbesondere der Vergleich zur korrespondierenden Volumenprobe von Interesse; für $\mathrm{Cr}_{2} \mathrm{AlC}$ finden sich entsprechende PACSpektren in Referenz [47], Gitterkonstanten sind in Referenz [4] gegeben.

Die Röntgenstrukturanalyse erfolgte anders als bei den hier untersuchten Volumenproben unter einem streifendem Einfall von $5^{\circ}$. Das so erhaltene Diffraktogramm der Dünnschichtprobe zeigt die typischen Reflexe der MAX-Phase $\mathrm{Cr}_{2} \mathrm{AlC}$. Die zugehörigen Gitterkonstanten lauten $a=2,862(5) \AA$ bzw. $c=12,81(2) \AA$ und stimmen mit denen der Volumenprobe überein (siehe Tabelle 5.1).

Die strukturelle Analyse wurde durch eine TEM-Untersuchung des Schichtquerschnittes ergänzt (siehe Abbildung 5.5). Es zeigt sich, dass die $\mathrm{Cr}_{2}$ AlC-Schicht $1,3(1) \mu \mathrm{m}$ dick und 
(a)

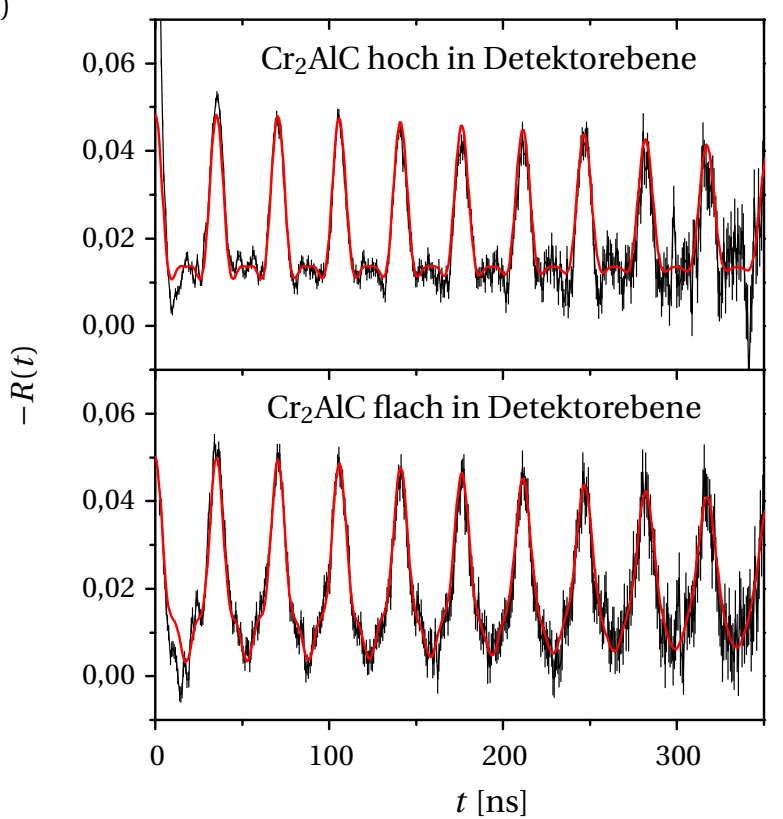

(b)

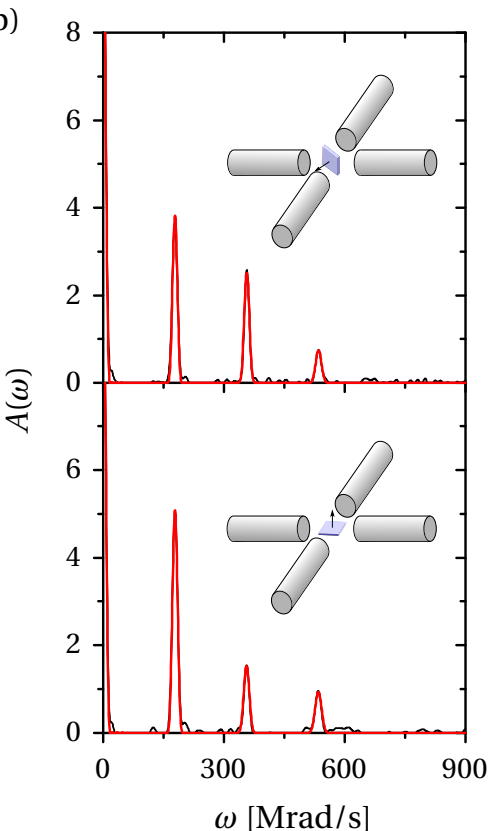

Abbildung 5.6: Störfunktion $R(t)$ (a) und dessen Fouriertransformation $A(\omega)$ (b) an der Cd-Sonde in $\mathrm{Cr}_{2} \mathrm{AlC}$-Dünnschichtproben bei Raumtemperatur in hoher und flacher Orientierung zur Detektorebene. Die schwarze Linie entspricht den Messdaten, die rote Linien einem Fit nach Gleichung 3.11. Die Probe wurde nach der Implantation für zwei Stunden bei $700^{\circ} \mathrm{C}$ in einer evakuierten Quarzampulle erhitzt. Ungefähr $75 \%$ der Cd-Sonden befinden sich auf dem Al-Platz der MAX-Phase, mit der für $\mathrm{Cr}_{2} \mathrm{AlC}$ charakteristischen Quadrupolfrequenz $v_{Q}=189$, 4(3) MHz bei einer Verteilungsbreite $\delta=0,9(1) \%$. Die Änderung der Übergangsamplituden mit der Orientierung relativ zur Detektorebene lässt auf eine Textur in der Probe schließen.

polykristalliner Natur ist. Die Körner erscheinen in der Dunkelfeldabbildung als längliche Kristallite mit einer Größe zwischen $100 \mathrm{~nm}$ und $600 \mathrm{~nm}$. Eine unmittelbare Vorzugsrichtung der Körner ist - auch anhand der Beugungsbilder - nicht zu erkennen. Die Oberfläche der Schicht ist im Vergleich zur Schichtdicke mit einer Rauheit im Bereich der Kristallitgröße relativ uneben; innherhalb der Schicht zeigen sich jedoch keinerlei Poren oder Risse.

\subsubsection{Elektrische Quadrupolwechselwirkung in Dünnschichtsystemen}

PAC-Messungen unmittelbar nach der Implantation der Cadmiumsonde zeigen ein für MAX-Phasen typisches, durch Implantationsschäden dominiertes Spektrum mit breiter Frequenzverteilung [44-47]. Isochrones Ausheilen der Probe für je eine Stunde bei $773 \mathrm{~K}$, $873 \mathrm{~K}$ und $973 \mathrm{~K}$ führt zu einer ansteigenden Rekristallisation der geschädigten Bereiche, wobei in der Störfunktion das für den A-Platz der MAX-Phasen typische Frequenztriplett sichtbar wird. Das schrittweise Ausheilen kann alternativ durch einmaliges Ausheilen für zwei Stunden bei 973K ersetzt werden, der Einfluss auf das PAC-Spektrum bleibt der Gleiche. Nach dem Ausheilen bei $973 \mathrm{~K}$ sind 75\% der Sonden auf dem A-Platz eingebaut, die 


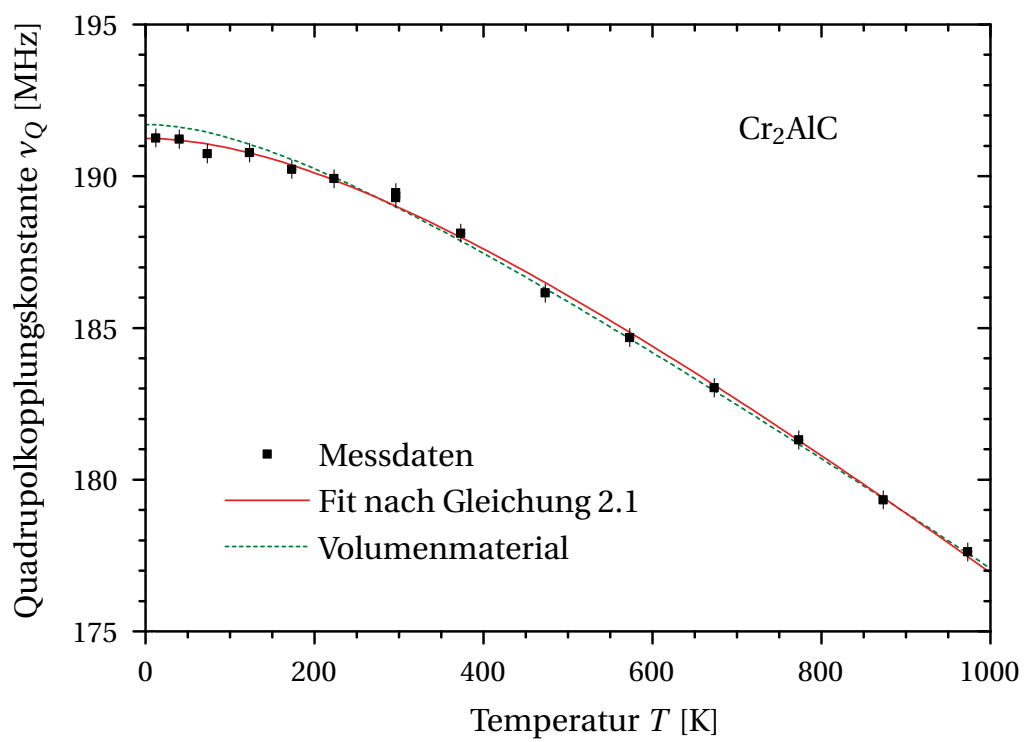

Abbildung 5.7: Temperaturabhängigkeit der Quadrupolkopplungskonstante $v_{Q}$ in $\mathrm{Cr}_{2} \mathrm{AlC}$-Dünnschichtproben. Die Messdaten im Dünnschichtsystem genügen, wie auch die Messdaten im Volumenmaterial [47], dem empirischen Zusammenhang aus Gleichung 2.1. Die entsprechenden Konstanten $B_{T}^{z z}$ und $C_{T}^{z z}$ unterscheiden sich im Rahmen des Fehlers nur gering.

restlichen Sonden befinden sich in einer Umgebung, die keinen dedizierten Feldgradienten aufweisen. Die Dämpfung der Störfunktion sinkt auf 0,9(1)\%, die Quadrupolfrequenz beträgt bei Raumtemperatur $v_{Q}=189,4$ (3) MHz. Das Maximum der Korrelation im CzjzekDiagramm liegt bei $\eta=0$ (nicht gezeigt), der Feldgradient ist also axialsymmetrisch. Das PAC-Spektrum einer solchen Probe ist in Abbildung 5.6 für zwei unterschiedliche Orientierungen relativ zur Detektorebene dargestellt. Anhand der Unterschiede beider Spektren wird deutlich, dass der implantierte Bereich eine Vorzugsrichtung der Kornorientierung aufweisen muss. Die Oberfläche der Proben zeigt nach der Temperaturbehandlung keine sichtbare Veränderung, weniger als $1 \%$ der radioaktiven Sonden verlassen die Schicht.

Wird die Ausheiltemperatur weiter auf über $1073 \mathrm{~K}$ erhöht, läuft die Schicht braun an, beginnt also zu degradieren. Ein zunehmender Teil der radioaktiven Sonden verlässt die Schicht und scheidet sich in der Quarzampulle ab, in der die Probe geheizt wird. Der Anteil der Sonden auf dem A-Platz sinkt folglich, die Dämpfung der Störfunktion bleibt aber konstant. Die Zersetzungsprodukte zeigen keinen eigenen Feldgradienten, sind also entweder von kubischer Kristallstruktur oder verhindern den Einbau des radioaktiven Indiums. Da die Zersetzungsprodukte nicht Gegenstand dieser Untersuchung sind, wird auf eine TEMAnalyse selbiger verzichtet.

\subsubsection{Temperaturabhängigkeit der Quadrupolkopplungskonstante}

Die Abhängigkeit des Feldgradienten von der Messtemperatur wird an einer Probe untersucht, die für zwei Stunden bei 973 K ausgeheilt wurde. Der gewählte Messbereich erstreckt 
Tabelle 5.3: Vergleich zwischen der Quadrupolkopplungskonstante $v_{Q}$ bei $T=0 \mathrm{~K}$ sowie den entsprechenden Koeffizienten $B_{T}^{z z}$ und $C_{T}^{z z}$, die den Temperaturverlauf nach Gleichung 2.1 beschreiben, an der Cd-Sonde in Volumen- und Dünnschichtproben aus $\mathrm{Cr}_{2} \mathrm{AlC}$. Die EFG-Verteilungsbreite $\delta$ zeigt keine Temperaturabhängigkeit.

\begin{tabular}{ccc}
\hline & Dünnschichtsystem & Volumenmaterial [47] \\
\hline$v_{Q}(0 \mathrm{~K})$ & $191,2(2) \mathrm{MHz}$ & $191,7(2) \mathrm{MHz}$ \\
$\delta$ & $0,9(1) \%$ & $0,9(1) \%$ \\
$B_{T}^{z z}$ & $12,1(1) \cdot 10^{-5} \mathrm{~K}^{-1}$ & $10,2(4) \cdot 10^{-5} \mathrm{~K}^{-1}$ \\
$C_{T}^{z z}$ & $620(120) \mathrm{K}$ & $337(55) \mathrm{K}$ \\
\hline
\end{tabular}

sich von $12 \mathrm{~K}$ bis hin zur maximalen Ausheiltemperatur. Die Dämpfung der Störfunktion bleibt über den gesamten Temperaturbereich konstant, lediglich die Quadrupolkopplungskonstante sinkt mit steigender Temperatur (siehe Abbildung 5.7). Zum Vergleich ist der Temperaturverlauf der Volumenprobe [47] hinterlegt; beide Verläufe sind nahezu identisch. Der Temperaturverlauf des Feldgradienten genügt dem empirischen Zusammenhang aus Gleichung 2.1, die aus dem Fit erhaltenen Konstanten $B_{T}^{z z}$ und $C_{T}^{z z}$ sowie die zu $T=0 \mathrm{~K}$ hin extrapolierte Quadrupolkopplungskonstante sind in Tabelle 2.1 denen der Volumenprobe gegenübergestellt. Im Rahmen des Fehlers unterscheiden sich die Parameter nur geringfügig. Die Unterschiede resultieren vermutlich aus dem bis $973 \mathrm{~K}$ beschränkten Messbereich der Dünnschichtprobe, was zu einer anderen Wichtung des gekrümmten Bereichs bei niedrigen Temperaturen führt.

Sowohl aus der bei Raumtemperatur gemessenen Störfunktion als auch aus der Temperaturabhängigkeit der Quadrupolwechselwirkung ergibt sich somit kein Hinweis auf eine interne Spannung im Dünnschichtsystem - weder aufgrund intrinsischer Defekte oder Spannungen, noch aufgrund thermisch induzierter Spannungen.

\subsection{Mischkristallsysteme}

Für die Untersuchung der Mischkristallsysteme werden die Legierungen der vorher untersuchten reinen MAX-Phasen herangezogen. Während die vollständige Mischbarkeit der Systeme ( $\left.\mathrm{Ti}_{1-x}, \mathrm{Nb}_{x}\right)_{2} \mathrm{AlC}$ [34] und $\mathrm{Ti}_{2} \mathrm{Al}\left(\mathrm{C}_{1-x}, \mathrm{~N}_{x}\right)$ [36] hinlänglich bekannt ist, fehlen entsprechende Experimente im System $\mathrm{Ti}_{2}\left(\mathrm{Al}_{1-x}, \mathrm{In}_{x}\right) \mathrm{C}$. Die Synthese und Charakterisierung dieses bisher unbekannten Systems wird nachfolgend für $x=0,5$ erstmals beschrieben. Die spezielle Wahl dieser Systeme ermöglicht am Beispiel von $\mathrm{Ti}_{2} \mathrm{AlC}$ eine systematische Untersuchung des Einflusses einer Mischung auf jedem der drei Gitterplätze der MAX-Phase - dem M-Platz, dem A-Platz und dem X-Platz. Als Erweiterung der Untersuchungen einer Mischung auf dem M-Platz wird zusätzlich das System $\left(\mathrm{Ti}_{1-x}, \mathrm{~V}_{x}\right)_{2} \mathrm{AlC}$ [35] herangezogen, sodass bei gleichbleibendem Einbauort der Einfluss des eingebauten Elements selbst untersucht werden kann. Der Anteil der ersetzten Atome bleibt in allen Experimenten konstant bei $x=0,5$. 


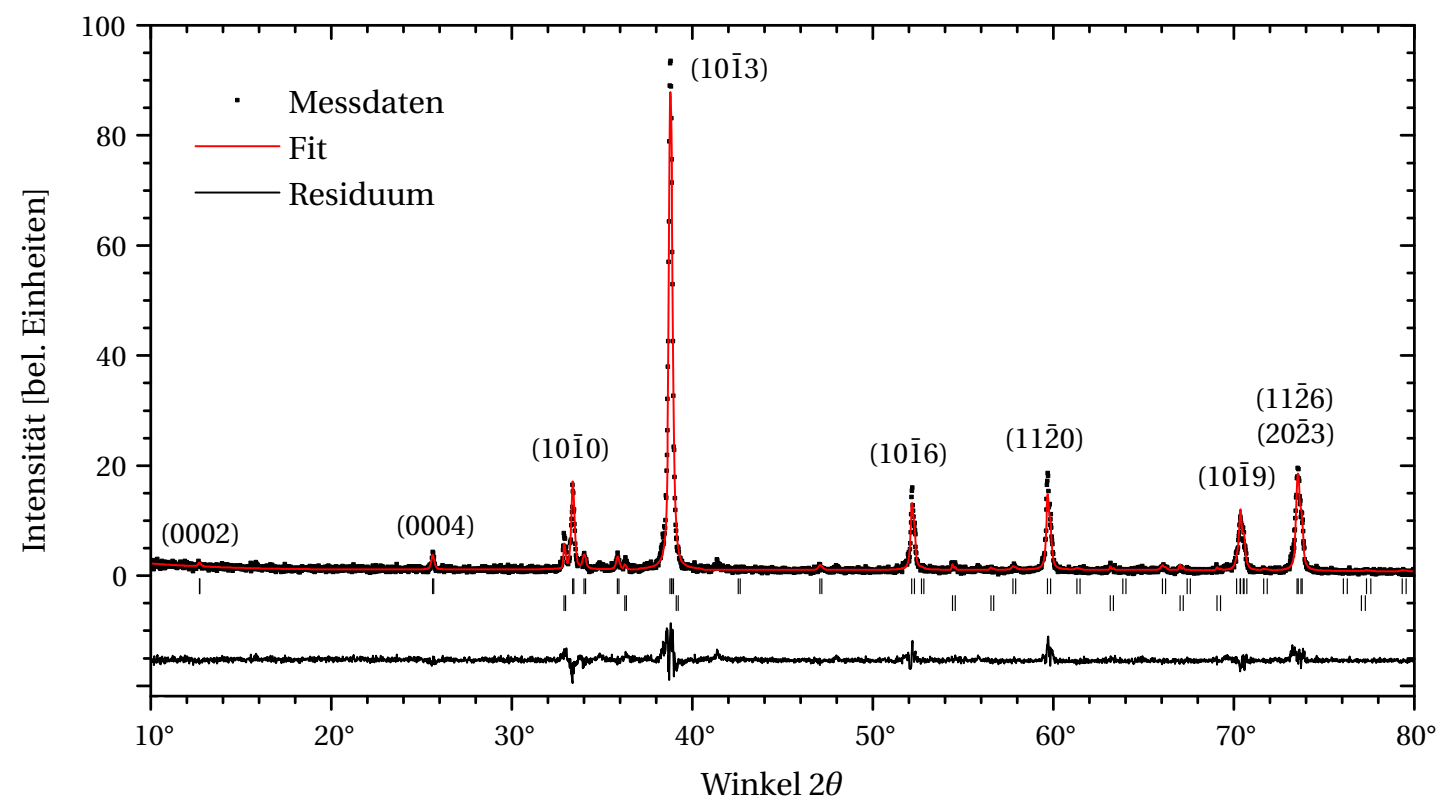

Abbildung 5.8: Röntgendiffraktogramm von $\mathrm{Ti}_{2}\left(\mathrm{Al}_{0,5}, \mathrm{In}_{0,5}\right) \mathrm{C}$ in $\theta-2 \theta$-Geometrie. Der Fit der Messdaten zeigt, dass in der Volumenprobe neben $\mathrm{Ti}_{2}\left(\mathrm{Al}_{0,5}, \mathrm{In}_{0,5}\right) \mathrm{C}$ auch metallisches Indium vorliegt. Die zugehörigen Massenanteile betragen 97(1) bzw. 3(1)\%. Unterhalb des Diffraktogramms sind die Reflexpositionen für $\mathrm{Ti}_{2}\left(\mathrm{Al}_{0,5}, \mathrm{In}_{0,5}\right) \mathrm{C}$ (obere Reihe) und Indium (untere Reihe) sowie das Residuum zwischen Messdaten und Fit eingezeichnet. Die durch den Fit ermittelten Gitterkonstanten der MAX-Phase betragen bei Raumtemperatur $a=3,093(1) \AA$ bzw. $c=13,871(2) \AA$.

\subsubsection{Synthese und Charakterisierung von $\mathrm{Ti}_{2}\left(\mathbf{A l}_{\mathbf{0}, 5}, \operatorname{In}_{\mathbf{0 , 5}}\right) \mathrm{C}$}

Die Synthese von $\mathrm{Ti}_{2}\left(\mathrm{Al}_{0,5}, \mathrm{In}_{0,5}\right) \mathrm{C}$ erfolgte ausgehend von elementaren Titan-, Aluminiumund Graphitpulvern (Alfa Aesar) sowie elementaren Indiumpellets (Cerag) im stöchiometrischen Verhältnis der Zielphase. Die verwendeten Pulver wiesen eine Reinheit von mindestens 99\% auf, die Pellets von mindestens 99,99\%. Partikel- und Pelletgröße lagen bei unter $50 \mu \mathrm{m}$ bzw. $3 \mathrm{~mm}$. Alle Pulver wurden mit einer Kugelmühle 14 Stunden vermischt, bevor die Indiumpellets untergemengt und das Gemisch in einem Rohrofen unter Argonfluss für zwei Stunden bei $1573 \mathrm{~K}$ vorreagiert wurde. Der Ofen wurde dabei mit $10 \mathrm{~K}$ pro Minute aufgeheizt. Die niederschmelzenden Indiumpellets reagierten in dieser Zeit vollständig mit dem Pulver zu einer homogenen, porösen Masse, die anschließend mit einem Mörser pulverisiert wurde. Dieses Pulver wurde in einen Hohlzylinder aus Graphit gefüllt, dessen Innenseite mit Graphitfolie ausgekleidet und mit Bornitrid-Spray beschichtet wurde. Ober- und Unterseite wurden mit zwei Graphitzylindern verschlossen, über die das Pulver während der Synthese komprimiert wurde.

Der eigentliche Sintervorgang erfolgte in einer Heißpresse unter einem Vakuum von 0,3 mbar. Der Graphitzylinder wurde hierfür mit $300 \mathrm{~K} / \mathrm{h}$ auf $1573 \mathrm{~K}$ erhitzt, eine Stunde vor Erreichen der Maximaltemperatur wurde zusätzlich die Presskraft innerhalb der verbleibenden Stunde auf 40 MPa erhöht. Diese Prozessparameter wurden für drei Stunden 
gehalten, bevor Druck und Temperatur wieder abgesenkt und die MAX-Phase ausgebaut wurde. Die Oberfläche des Presslings war nach dem Sintern noch mit Teilen der Graphitfolie verbunden, welche anschließend abgeschliffen wurde.

Eine quantitative Phasenanalyse des Presslings mittels Röntgendiffraktometrie ergibt einen Massenanteil der anvisierten Phase $\mathrm{Ti}_{2}\left(\mathrm{Al}_{0,5}, \mathrm{In}_{0,5}\right) \mathrm{C}$ von $97 \%$. Der restliche Anteil ist metallischen Indiumausscheidungen zuzuordnen. Die Gitterkonstanten des Mischkristalls betragen $a=3,093(1) \AA$ bzw. $c=13,871$ (2) $\AA$ (siehe Tabelle 5.1). Das gemessene Diffraktogramm ist mit dem zugehörigen Fit in Abbildung 5.8 gezeigt. Die Vorzugsrichtung der Körner fällt sehr gering aus. Der bei $12,8^{\circ}$ nahezu verschwindende Reflex ist charakteristisch für einen MAX-Phasen-Mischkristall mit fünfzigprozentiger Durchmischung auf dem A-Platz und eine Konsequenz der verminderten Kristallsymmetrie; in reinen MAX-Phasen trägt dieses Reflex erheblich zum Spektrum bei. Da keine zusätzlichen Reflexe beobachtet werden können, liegt keine Fernordnung der Atome auf dem A-Platz vor. So würden bei einer Überstruktur beispielsweise entlang der c-Achse des Kristalls signifikante (0001)und (0003)-Reflexe erwartet werden, die beide nicht zu sehen sind. Es handelt sich also um einen ungeordneten Subsitutionsmischkristall.

Die Dichtebestimmung nach dem archimedischen Prinzip ergibt 5,079(10) $\mathrm{g} / \mathrm{cm}^{3}$, was knapp unter der theoretischen Dichte von $5,165 \mathrm{~g} / \mathrm{cm}^{3}$ liegt. Unter Berücksichtigung der zusätzlich enthaltenen Inidumphase ergibt sich so eine Porosität der Probe von $3 \%$.

\subsubsection{Elektrische Quadrupolwechselwirkung in Mischkristallsystemen}

PAC-Messungen nach der Implantation der Cadmiumsonde zeigen in allen Mischkristallen wie auch bei den Dünnschichtsystemen das für MAX-Phasen typische, durch Implantationsschäden dominierte Spektrum mit breiter Frequenzverteilung. Isochrones Ausheilen der Proben für je eine Stunde bei 773 K, 873 K, 973 K, 1073 K und 1173 K führt zu einer ansteigenden Rekristallisation der geschädigten Bereiche. In den Störfunktionen der Mischkristalle wird dabei jeweils ein für den A-Platz der MAX-Phasen typisches Frequenztriplett zunehmend sichtbar, gleichzeitig sinkt die Dämpfung der Störfunktion. Während des Ausheilens treten weniger als 1\% der radioaktiven Sonden aus den MAX-Phasen aus; bei hohen Temperaturen bildet sich in der umschließenden Quarzampulle ein gelb-bräunlicher Niederschlag. Anhand der PAC-Spektren lassen sich keine Zersetzungserscheinungen erkennen.

Wie bei anderen PAC-Experimenten üblich, kann auch hier der Ausheilvorgang durch fünfstündiges Heizen bei 1173K abgekürzt werden, das erhaltene PAC-Spektrum bleibt das Gleiche. Auf den ersten Blick scheinen die Implantationsschäden auf diese Weise bereits minimiert zu sein, wie sich aber später bei den Hochtemperaturmessungen herausstellen wird, verbessert sich die Qualität der Spektren über die lange Mess- und somit Heizdauern weiter. Quantitative Angaben der Dämpfung stellen deswegen nachfolgend jeweils das bei Raumtemperatur gemessene Minimum nach der Hochtemperaturmessung dar.

Die bei Raumtemperatur gemessenen PAC-Spektren der vier untersuchten Mischkristallsysteme sind zusammen mit den Spektren der reinen MAX-Phasen in Abbildung 5.9 bis 5.12 gezeigt. Alle Spektren lassen sich - abgesehen von einem Beitrag metallischen Indiums in $\mathrm{Ti}_{2}\left(\mathrm{Al}_{0,5}, \mathrm{In}_{0,5}\right) \mathrm{C}$ - mit einem einzigen Anteil $f_{i}$ durch Gleichung 3.11 beschreiben; 
(a)

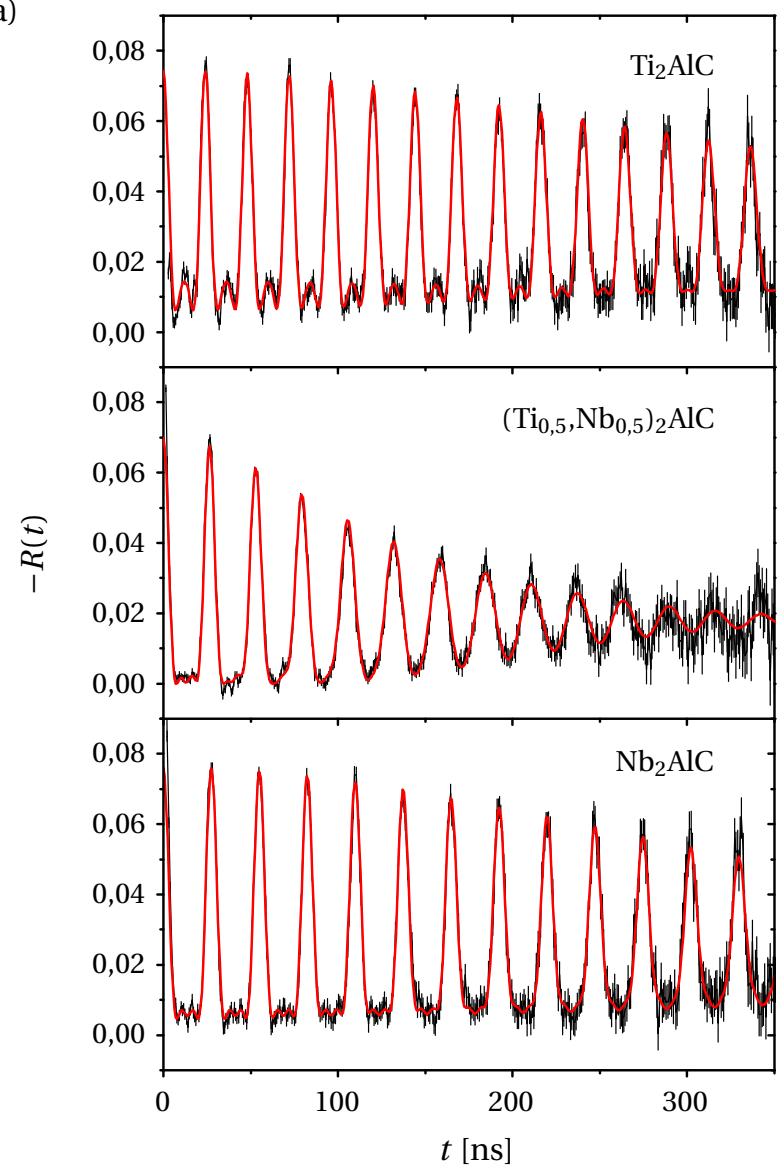

(b)

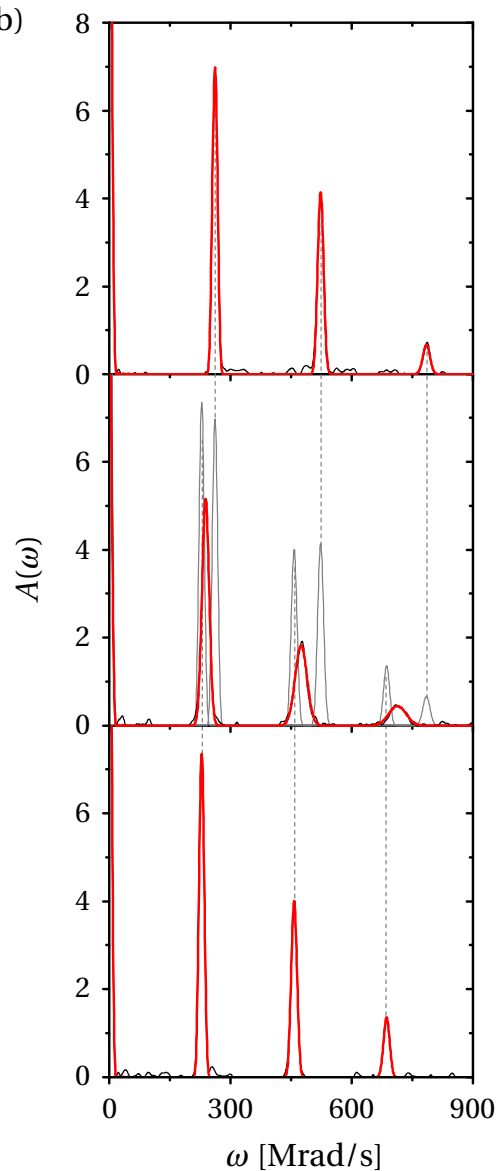

Abbildung 5.9: Störfunktionen $R(t)$ (a) und deren Fouriertransformierten $A(\omega)$ (b) an der Cd-Sonde im System $\left(\mathrm{Ti}_{1-x}, \mathrm{Nb}_{x}\right)_{2} \mathrm{AlC}$ bei Raumtemperatur. Die Frequenztripletts der reinen Phasen sind dem Triplett des Mischkristalls mit $x=0,5$, entsprechend einer Quadurpolkopplungskonstante von $v_{Q}=251,6(5) \mathrm{MHz}$, grau überlagert. Die dadurch sichtbare Abweichung von der Mittelposition ist ein Charakteristikum aller untersuchten Mischkristallsysteme der MAX-Phasen.

es bilden sich insbesondere keine Schwebungen auf der Störfunktion aus, die auf zwei nahe beieinander liegende, unterscheidbare Quadrupolfrequenzen, wie die der reinen MAXPhasen, hindeuten würden. Das gesamte Implantationsvolumen besteht also aus einem einzigen, homogenen MAX-Phasen-Mischkristall. Die zugehörigen Quadrupolkopplungskonstanten liegen in allen Systemen innerhalb, keineswegs aber genau mittig zwischen den Kopplungskonstanten der reinen Phasen. Dies wird besonders in den Fouriertransformierten der Störfunktionen augenscheinlich.

Die Frequenzverteilung ist in allen Mischkristallen stark verbreitert, in $\operatorname{Ti}_{2}\left(\mathrm{Al}_{0,5}, \mathrm{In}_{0,5}\right) \mathrm{C}$ sogar so stark, dass sie über die Verteilungen der reinen Phasen hinausragt (siehe Abbildung 5.11b). Die nach Gleichung 3.11 ermittelte Dämpfung der Spektren liegt zwischen $1,3 \%$ für $\mathrm{Ti}_{2} \mathrm{Al}\left(\mathrm{C}_{0,5}, \mathrm{~N}_{0,5}\right)$ und $5,4 \%$ für $\left(\mathrm{Ti}_{0,5}, \mathrm{~V}_{0,5}\right)_{2} \mathrm{AlC}$. Typische Dämpfungen reiner MAX- 
(a)

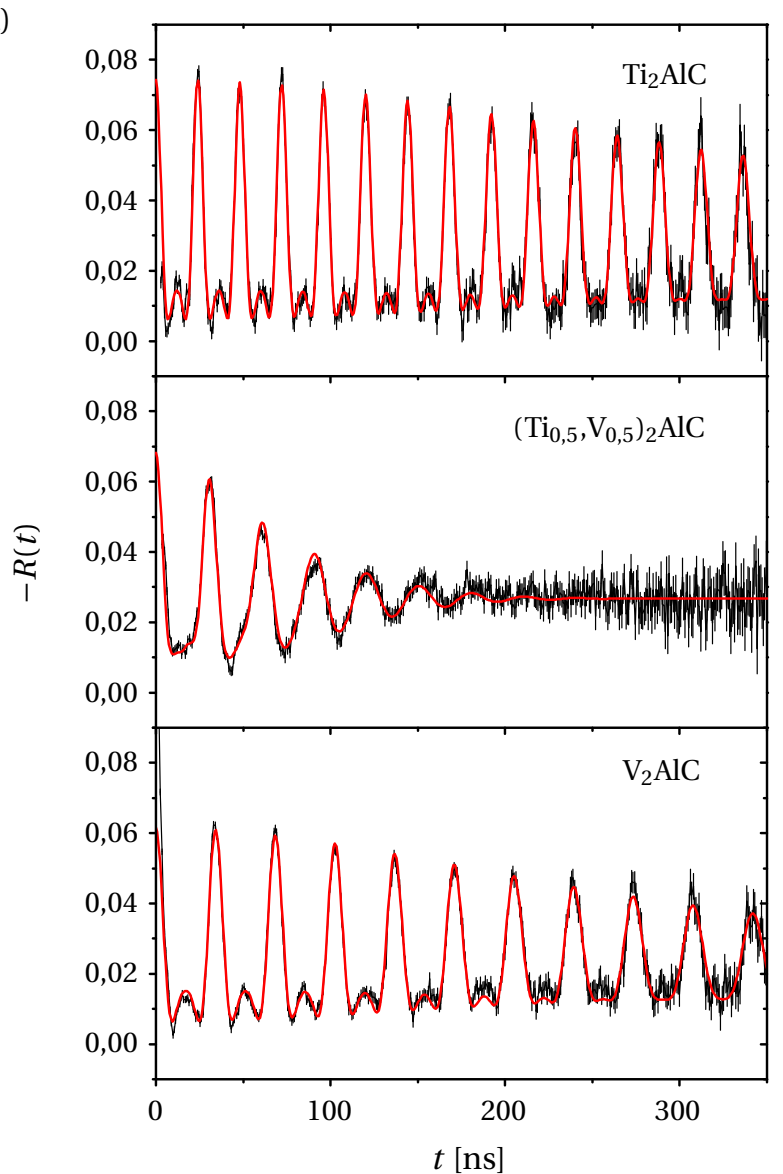

(b)

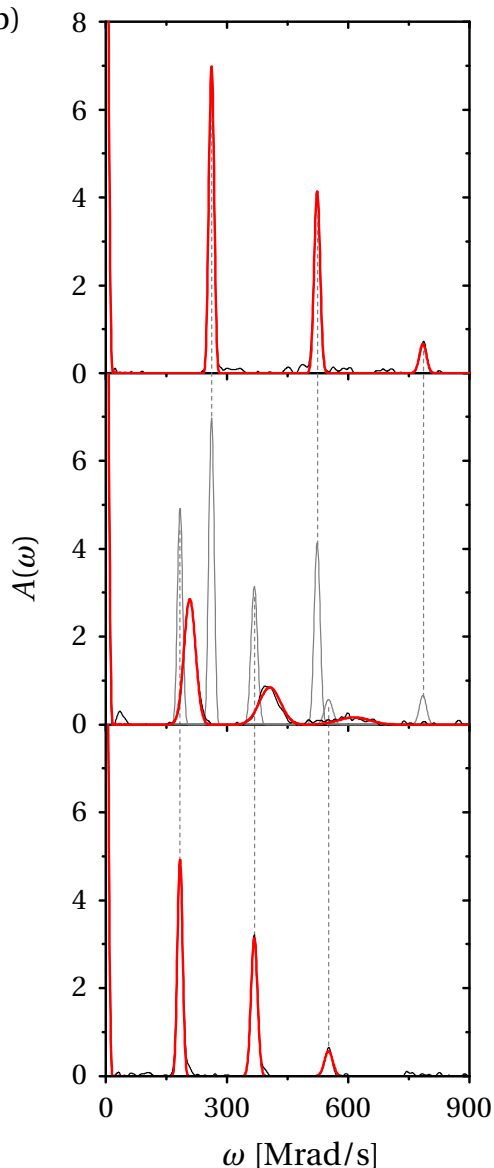

Abbildung 5.10: Störfunktionen $R(t)$ (a) und deren Fouriertransformierten $A(\omega)$ (b) an der CdSonde im System $\left(\mathrm{Ti}_{1-x}, \mathrm{~V}_{x}\right)_{2} \mathrm{AlC}$ bei Raumtemperatur. Bei Substituion der Hälfte der Titanatome durch Vanadium beträgt die Quadrupolkopplungskonstante $v_{Q}=$ 219(1) MHz. Das Spektrum von $\mathrm{V}_{2} \mathrm{AlC}$ wurde aus Referenz [47] entnommen.

Phasen liegen im Bereich von einem Prozent. Das Maximum der Korrelation im CzjzekDiagramm liegt bei allen Mischkristallen bei $\eta=0$ (nicht gezeigt), die Axialsymmetrie bleibt also - im Terminus von Butz [167] - erhalten, wenngleich analog zu $v_{Q}$ eine Verbreiterung der Verteilung von $\eta$ auftritt, die sich beim Fit mit Gleichung 3.11 in einem scheinbar von null verschiedenen Asymmetrieparameter äußert (vgl. Kapitel 3.1.4).

\subsubsection{Temperaturabhängigkeit der Quadrupolkopplungskonstante}

Die Temperaturabhängigkeit der Quadrupolkopplungskonstante kann im Gegensatz zum bei $1073 \mathrm{~K}$ degradierenden Dünnschichtsystem über einen erweiterten Temperaturbereich von $12 \mathrm{~K}$ bis $1173 \mathrm{~K}$ vermessen werden. Die Verläufe aller vier Mischkristallsysteme sind in Abbildung 5.13 denen der reinen Phasen gegenüber gestellt. Vier der fünf reinen Systeme wurden von Jürgens [47] bereits charakterisiert, für $\mathrm{Ti}_{2} \mathrm{AlN}$ wurde der Verlauf im Rahmen 
(a)

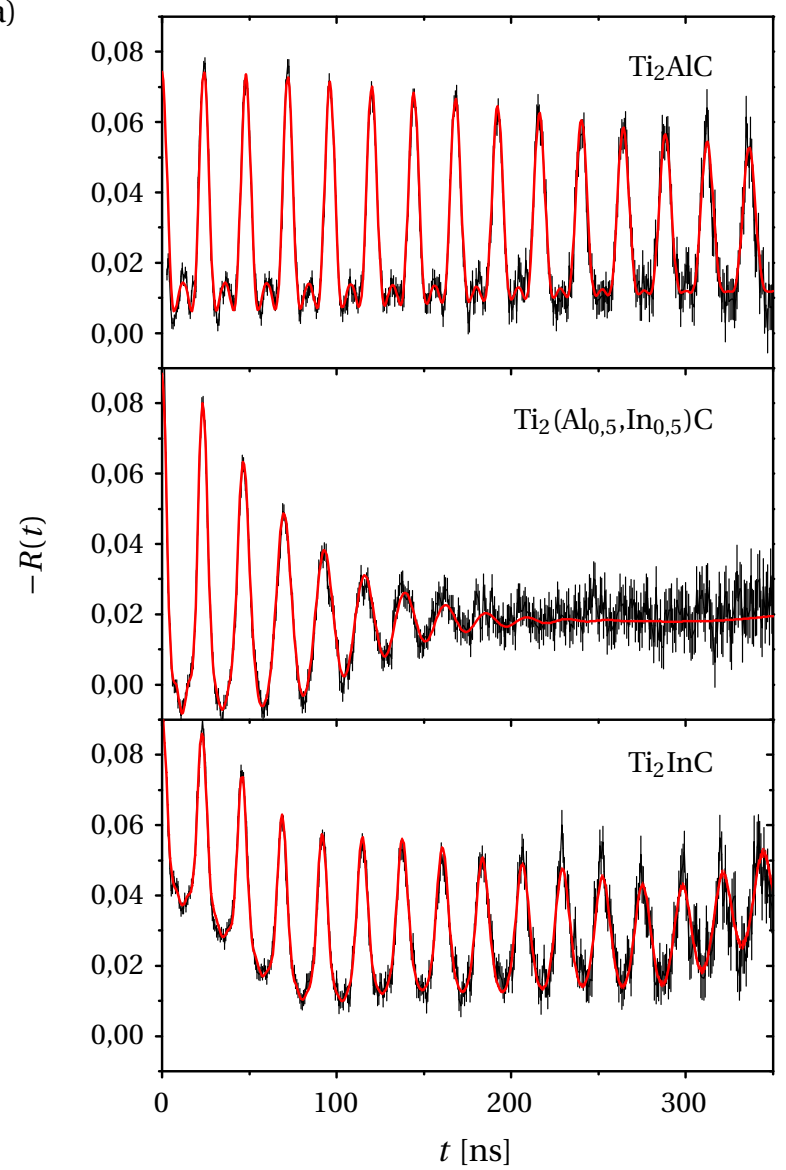

(b)

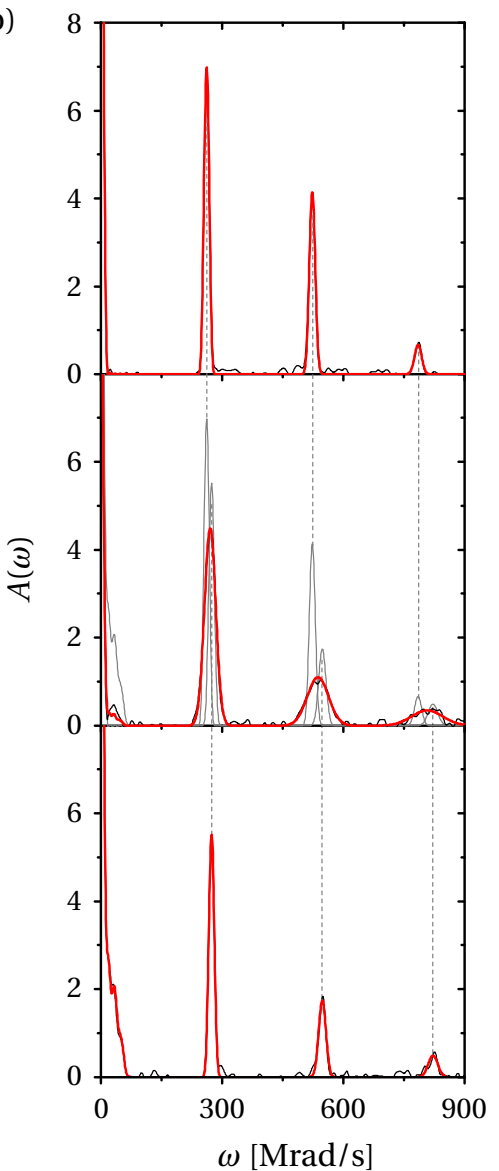

Abbildung 5.11: Störfunktionen $R(t)$ (a) und deren Fouriertransformierten $A(\omega)$ (b) an der CdSonde im System $\mathrm{Ti}_{2}\left(\mathrm{Al}_{1-x}, \mathrm{In}_{x}\right) \mathrm{C}$ bei Raumtemperatur. Für $x=0,5$ beträgt die Quadrupolkopplungskonstante 286,1(8) MHz. Zusätzlich zum Frequenztriplett der MAX-Phasen ist ein weiteres Triplett bei niedrigen Frequenzen sichtbar, das metallischen Indium zugeordnet werden kann.

dieser Arbeit analog vermessen.

Der Verlauf liegt für alle Mischkristalle innerhalb derer der reinen Phasen. Die Messdaten lassen sich analog zu den reinen Phasen hervorragend mit Gleichung 2.1 anpassen. Die entsprechenden Konstanten $B_{T}^{z z}$ und $C_{T}^{z z}$ sowie die zu $T=0 \mathrm{~K}$ hin extrapolierte Quadrupolkopplungskonstante sind in Tabelle 5.4 aufgelistet. Wie die Quadrupolkopplungskonstanten liegen auch die Konstanten $B_{T}^{z z}$ und $C_{T}^{z z}$ zwischen denen der reinen Phasen, es folgen ähnliche Verläufe innerhalb eines Mischkristallsystems. In vielen Systemen schneiden sich die Temperaturverläufe bei einer spezifischen Temperatur $T_{\text {eq }}$, lediglich in $\operatorname{Ti}_{2}\left(\mathrm{Al}_{0,5}, \mathrm{In}_{0,5}\right) \mathrm{C}$ divergieren die Verläufe.

Die Dämpfung sinkt mit zunehmender Messtemperatur um 0,4 bis 0,7 Prozentpunkte ab, was maßgeblich auf die fortschreitende Rekristallisation der durch den Ionenstrahl geschädigten Bereiche zurückzuführen ist, da die verminderte Dämpfung bei einer an- 
(a)

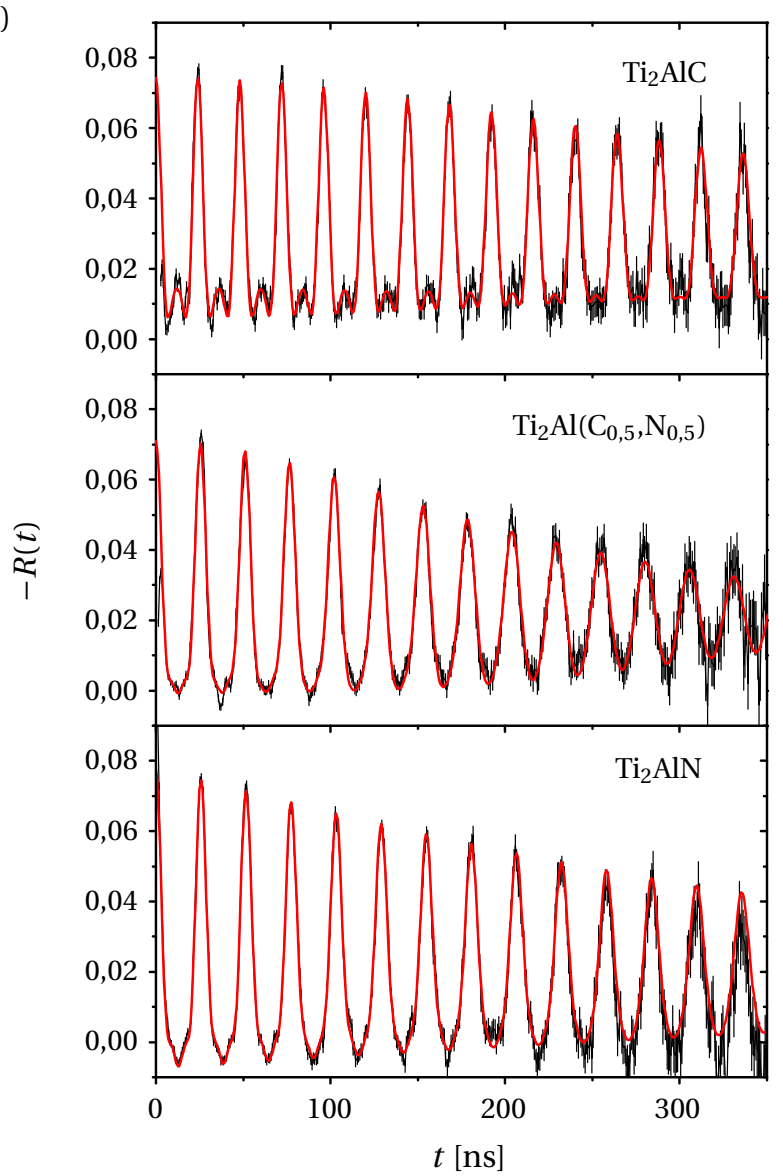

(b)

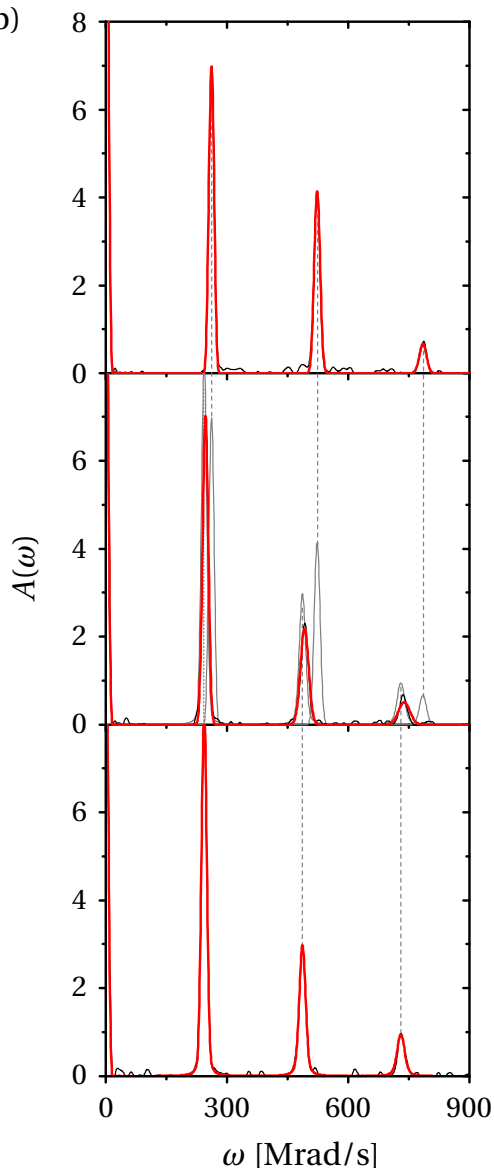

Abbildung 5.12: Störfunktionen $R(t)$ (a) und deren Fouriertransformierten $A(\omega)$ (b) an der CdSonde im System $\mathrm{Ti}_{2} \mathrm{Al}\left(\mathrm{C}_{1-x}, \mathrm{~N}_{x}\right)$ bei Raumtemperatur. Die Substition der Hälfte der Kohlenstoffatome durch Stickstoff führt zu einer Quadrupolkopplungskonstante von $v_{Q}=261,1(5) \mathrm{MHz}$.

schließenden Raumtemperaturmessung größtenteils erhalten bleibt. So steigt die Dämpfung nach der Hochtemperaturmessung in den Systemen $\left(\mathrm{Ti}_{0,5}, \mathrm{Nb}_{0,5}\right)_{2} \mathrm{AlC},\left(\mathrm{Ti}_{0,5}, \mathrm{~V}_{0,5}\right)_{2} \mathrm{AlC}$ und $\operatorname{Ti}_{2}\left(\mathrm{Al}_{0,5}, \mathrm{In}_{0,5}\right) \mathrm{C}$ geringfügig um 0,1 Prozentpunkte, auf die in Tabelle 5.4 angegebene Werte, an. Für $\mathrm{Ti}_{2} \mathrm{Al}\left(\mathrm{C}_{0,5}, \mathrm{~N}_{0,5}\right)$ ist diese Dynamik leicht erhöht, hier ergibt sich eine Dämpfung von 1,1(1)\% bei $T=1173 \mathrm{~K}$ gegenüber 1,3(1)\% bei der anschließenden Raumtemperaturmessung. Interessant ist hierbei weniger die Dynamik als die Tatsache, dass bei $T_{\text {eq }}$ nahezu die Dämpfung der reinen Phasen von $0,9(1) \%^{a}$ erreicht wird.

\subsubsection{Vergleich zu reinen MAX-Phasen}

Die graphische Auftragung der Quadrupolkopplungskonstanten und der Gitterkonstanten aus Tabellen 5.4 bzw. 5.1 als Funktion des Anteils $x$ der untersuchten Mischkristallsyste-

${ }^{a}$ In der reinen MAX-Phase $\mathrm{Ti}_{2} \mathrm{AlN}$ steigt die Dämpfung nach der Hochtemperaturmessung nicht mehr an. 

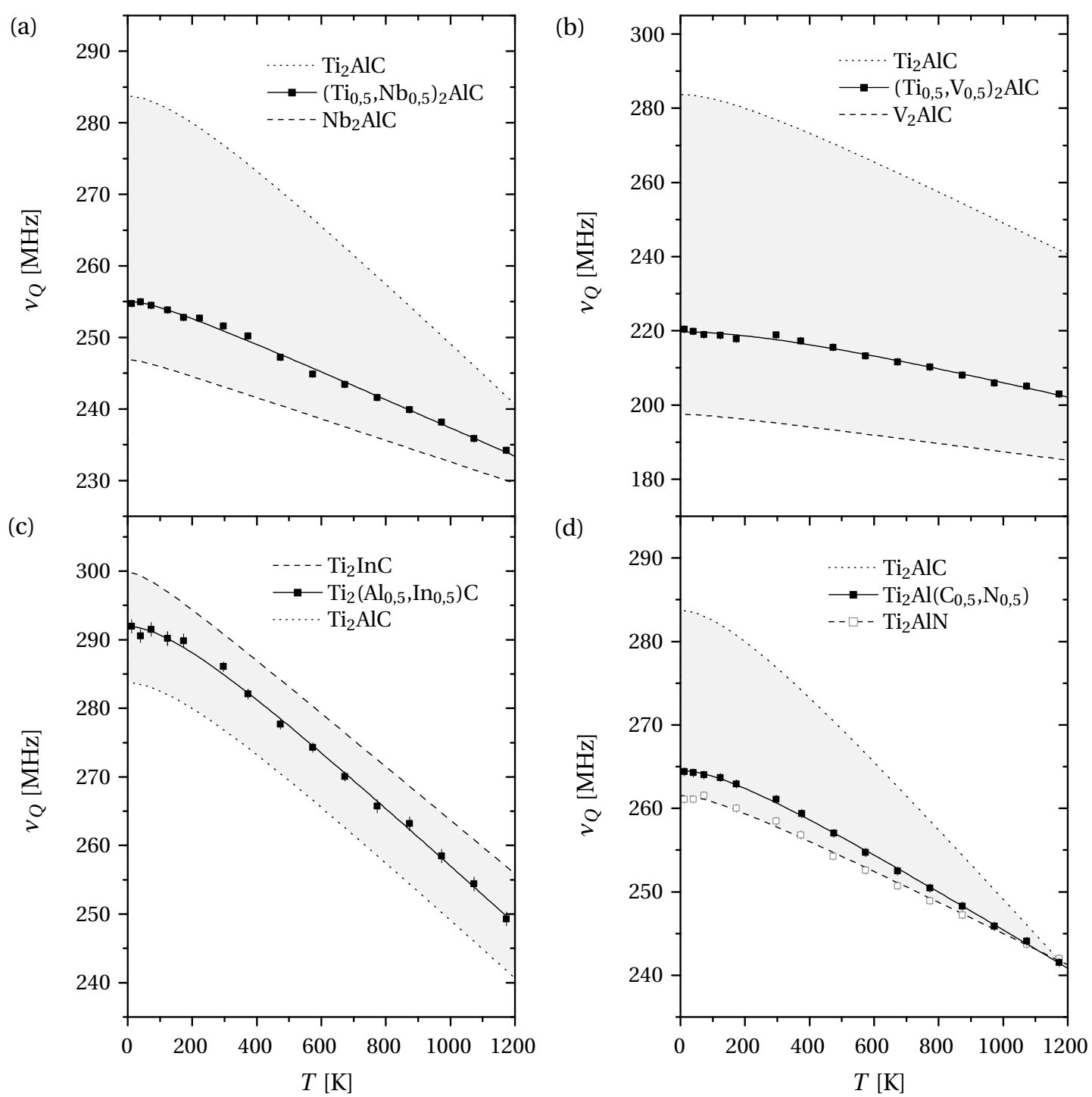

Abbildung 5.13: Temperaturabhängigkeit der Quadrupolkopplungskonstante $v_{Q}$ an der CdSonde in den Mischkristallsystemen $\left(\mathrm{Ti}_{1-x}, \mathrm{Nb}_{x}\right)_{2} \mathrm{AlC}$ (a), $\left(\mathrm{Ti}_{1-x}, \mathrm{~V}_{x}\right)_{2} \mathrm{AlC}$ (b), $\mathrm{Ti}_{2}\left(\mathrm{Al}_{1-x}, \mathrm{In}_{x}\right) \mathrm{C}$ (c) und $\mathrm{Ti}_{2} \mathrm{Al}\left(\mathrm{C}_{1-x}, \mathrm{~N}_{x}\right)$ (d). Die Messdaten in den Mischkristallen genügen, wie auch die Messdaten an der Cadmiumsonde in reinen MAX-Phasen [47], dem empirischen Zusammenhang aus Gleichung 2.1. Der entsprechende Fit ist jeweils als Linie eingezeichnet. Der Temperaturverlauf von $v_{Q}$ liegt jeweils innerhalb des Verlaufs der reinen Phasen. In vielen Systemen liefert eine Extrapolation der Daten eine spezifische Temperatur $T_{\text {eq }}$, bei der sich die Temperaturverläufe aller $x$ schneiden ( $1600 \mathrm{~K}$ (a), $3000 \mathrm{~K}$ (b) und $1200 \mathrm{~K}$ (d)). Die experimentellen Temperaturverläufe der reinen Phasen wurden, falls möglich, aus Referenz [47] entnommen. Im Falle von $\mathrm{Ti}_{2} \mathrm{AlN}$ wurde der entsprechende Verlauf separat gemessen. 


\section{Kapitel 5 Experimentelle Ergebnisse}

Tabelle 5.4: Quadrupolkopplungskonstante $v_{Q}$ bei $T=0 \mathrm{~K}$ und die minimale Verteilungsbreite $\delta$ des EFGs an der Cd-Sonde in reinen MAX-Phasen und deren Mischkristalle sowie die entsprechenden Koeffizienten $B_{T}^{z z}$ und $C_{T}^{z z}$, die den Temperaturverlauf nach Gleichung 2.1 beschreiben. Im Wesentlichen finden sich alle Parameter der Mischkristalle innerhalb der von den reinen Phasen vorgegeben Schranken wieder, lediglich die Verteilungsbreite zeigt eine systematische Vergrößerung in den Mischkristallen.

\begin{tabular}{ccccc}
\hline & $v_{Q}(0 \mathrm{~K})[\mathrm{MHz}]$ & $\delta[\%]$ & $B_{T}^{z z}\left[10^{-5} \mathrm{~K}^{-1}\right]$ & $C_{T}^{z z}[\mathrm{~K}]$ \\
\hline $\mathrm{Ti}_{2} \mathrm{AlC}[47]$ & $283,7(2)$ & $0,9(1)$ & $15,5(3)$ & $271(25)$ \\
$\left(\mathrm{Ti}_{0,5}, \mathrm{Nb}_{0,5}\right)_{2} \mathrm{AlC}$ & $255,0(3)$ & $2,8(2)$ & $7,8(3)$ & $130(50)$ \\
$\mathrm{Nb}_{2} \mathrm{AlC}[47]$ & $246,9(3)$ & $0,9(1)$ & $6,1(1)$ & $53(27)$ \\
\hline$\left(\mathrm{Ti}_{0,5}, \mathrm{~V}_{0,5}\right)_{2} \mathrm{AlC}$ & $219,7(4)$ & $5,4(2)$ & $10(2)$ & $600(300)$ \\
$\mathrm{V}_{2} \mathrm{AlC}[47]$ & $197,5(2)$ & $1,6(1)$ & $5,8(2)$ & $132(35)$ \\
\hline $\mathrm{Ti}_{2}\left(\mathrm{Al}_{0,5}, \mathrm{In}_{0,5}\right) \mathrm{C}$ & $291,9(4)$ & $4,7(2)$ & $15,0(7)$ & $250(60)$ \\
$\mathrm{Ti}_{2} \mathrm{InC}[47]$ & $299,8(5)$ & $0,8(1)$ & $13,1(3)$ & $88(27)$ \\
\hline $\mathrm{Ti}_{2} \mathrm{Al}_{\left(\mathrm{C}_{0,5}, \mathrm{~N}_{0,5}\right)}$ & $264,5(2)$ & $1,3(1)$ & $9(2)$ & $240(40)$ \\
$\mathrm{Ti}_{2} \mathrm{AlN}$ & $261,5(3)$ & $0,9(1)$ & $7,3(4)$ & $150(60)$ \\
\hline
\end{tabular}

(a)

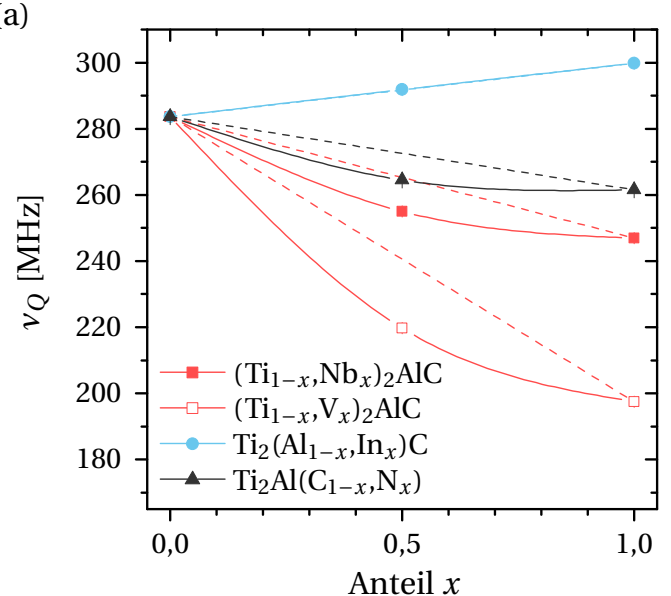

(b)

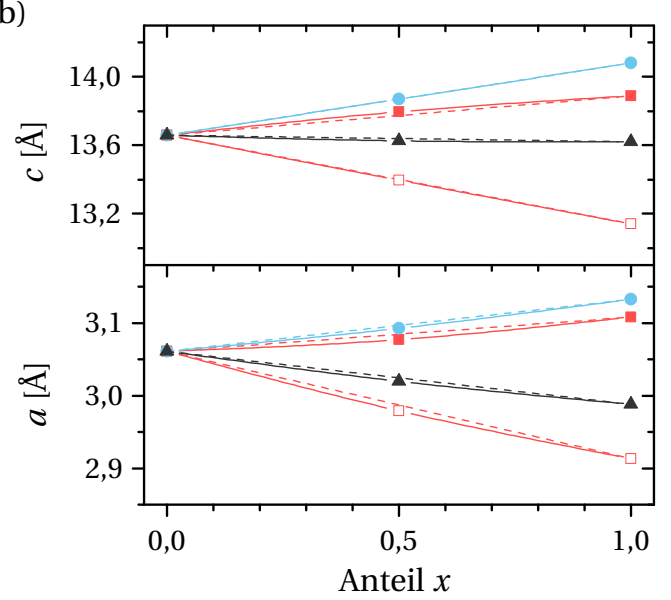

Abbildung 5.14: Quadrupolkopplungskonstanten $v_{Q}$ an der Cd-Sonde (a) und Gitterkonstanten $a$ bzw. $c$ (b) bei unterschiedlichen Anteilen $x$ der untersuchten Mischkristallsysteme (siehe Tabellen 5.4 und 5.1). Die gestrichelten Linien entsprechen einer linearen Interpolation zwischen den jeweiligen Werten der Endkomponenten, die durchgezogenen Linien einer Interpolation aller Messwerte mit stückweisen Polynomen. Im Falle der Gitterkonstanten ist die Abweichung von der linearen Interpolation gering, die Vegard'sche Regel also zutreffend. Anders die Quadrupolkopplungskonstante, die außer bei $\mathrm{Ti}_{2}\left(\mathrm{Al}_{1-x}, \mathrm{In}_{x}\right) \mathrm{C}$ starke Abweichungen von der linearen Interpolation zeigt. Die Gitterkonstanten für $\mathrm{V}_{2} \mathrm{AlC}$ wurden aus Referenz [4] entnommen. 


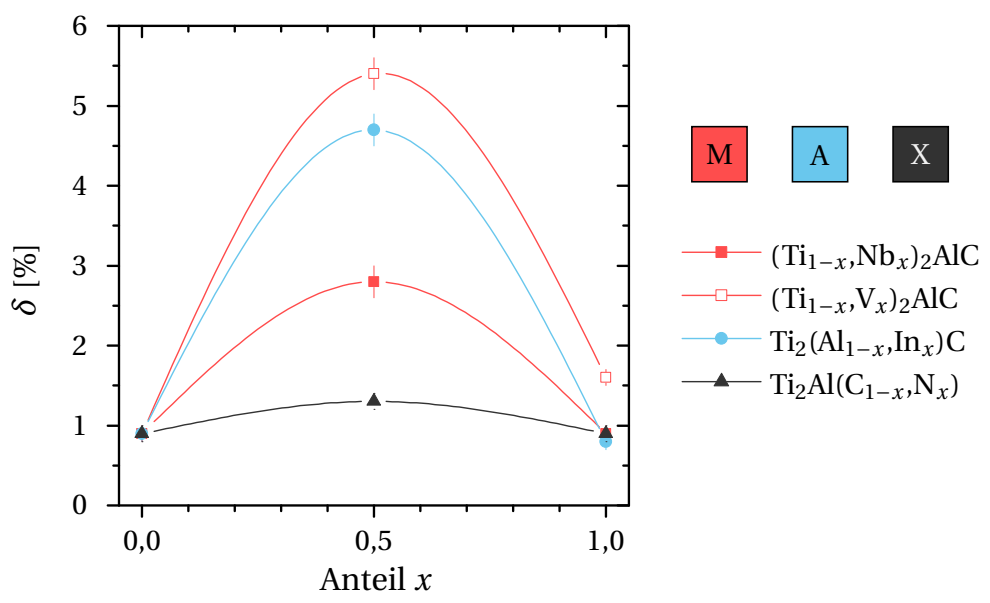

Abbildung 5.15: Verteilungsbreite des EFGs $\delta$ bei unterschiedlichen Anteilen $x$ der untersuchten Mischkristallsysteme (siehe Tabelle 5.4). Zur besseren Visualisierung wurden die Messwerte mit stückweisen Polynomen interpoliert. Auffällig ist die vergleichsweise kleine Verteilungsbreite bei fünfzigprozentiger Substitution von Kohlenstoff durch Stickstoff bei gleichzeitig relativ großem Unterschied der Quadrupolkopplungskonstanten der reinen MAX-Phasen; eine Substitution von Aluminum durch Indium führt hingegen zu einer massiven Verbreiterung bei kleinerem Unterschied der entsprechenden Quadrupolkopplungskonstanten.

me zeigt deutlich, wie unterschiedlich sich beide Größen beim Mischen verhalten (siehe Abbildung 5.14). Einerseits ändern sich die Gitterkonstanten der Mischkristalle im Einklang mit der Vegard'schen Regel näherungsweise linear mit dem Anteil $x$, andererseits ist - $\mathrm{Ti}_{2}\left(\mathrm{Al}_{1-x}, \mathrm{In}_{x}\right) \mathrm{C}$ ausgenommen - die Abweichung zwischen gemessener Quadrupolkopplungskonstante und linearer Interpolation der reinen Phasen offensichtlich. Die Quadrupolkopplungskonstante verhält sich also in Mischkristallen im Allgemeinen nicht gemäß der Vegard'schen Regel.

Die beobachteten Abweichungen sind reell, insbesondere sind sie kein Artefakt der $\eta$ Verteilung und auch kein statistischer Effekt des geringen Implantationsvolumens. Der durch die $\eta$-Verteilung eingebrachte Fehler in der Bestimmung von $v_{Q}$ ist zwar schwierig exakt zu quantifizieren, kann aber im Extremfall von $\left(\mathrm{Ti}_{0,5}, \mathrm{~V}_{0,5}\right)_{2} \mathrm{AlC}$ keinesfalls $4 \mathrm{MHz}$ übersteigen und würde die Abweichung noch verstärken [164]. In den anderen Systemen ist dieser Fehler kleiner, in $\mathrm{Ti}_{2}\left(\mathrm{Al}_{1-x}, \mathrm{In}_{x}\right) \mathrm{C}$ führt er eher zu einer leichten Abweichung vom scheinbar linearen Verhalten. Der Einfluss des Implantationsvolumens kann deshalb ausgeschlossen werden, weil die durchgeführten Messreihen an jeweils drei unterschiedlichen Probenstücken immer das gleiche Ergebnis liefern.

Die gleiche Darstellung der Dämpfung als Funktion des Anteils $x$ in Abbildung 5.15 verdeutlicht den höchst unterschiedlichen Einfluss des Mischens auf die Verteilungsbreite des Feldgradienten in verschiedenen Mischkristallsystemen. So werden einerseits für $\left(\mathrm{Ti}_{1-x}, \mathrm{Nb}_{x}\right)_{2} \mathrm{AlC},\left(\mathrm{Ti}_{0,5}, \mathrm{~V}_{0,5}\right)_{2} \mathrm{AlC}$ und $\mathrm{Ti}_{2} \mathrm{Al}\left(\mathrm{C}_{1-x}, \mathrm{~N}_{x}\right)$ bei stark bzw. schwach unterschiedlichen Quadrupolfrequenzen der beteiligten reinen Phasen große bzw. kleine Dämpfungen beobachtet, andererseits zeigt sich für $\mathrm{Ti}_{2}\left(\mathrm{Al}_{1-x}, \mathrm{In}_{x}\right) \mathrm{C}$ schon bei relativ kleinem Unter- 
schied eine extrem große Dämpfung. Die Implikationen dieser und der anderen hier genannten Beobachtung werden in Kapitel 6 diskutiert.

Die kubische Interpolation der Messdaten in den Abbildungen 5.14 und $5.15 \mathrm{kommt}$ dem tatsächlichen Verlauf der Quadrupolkopplungskonstante und auch der Dämpfung vermutlich recht nahe, da aus anderen Experimenten bekannt ist, dass sich beide Verläufe für kleine Konzentrationen linear ändern, sich über den gesamten Konzentrationsbereich stetig verhalten und im Falle der Dämpfung bei 50-prozentiger Mischung maximal werden [97, 103-106]. 


\section{Kapitel 6}

\section{Diskussion}

Nachfolgend werden die theoretischen Ergebnisse aus Kapitel 4 sowie die experimentellen Ergebnissen aus Kapitel 5 diskutiert. Um zunächst zu verstehen, warum und wie sich der Feldgradient mit der Dehnung verändert, wird zuerst die Ursache der Gradient-elastischen Konstanten anhand der elektronischen Zustandsdichten evaluiert. Bei allen weiteren Analysen ist es von zentraler Bedeutung, inwieweit den berechneten Gradient-elastischen Konstanten vertraut werden kann. Aus diesem Grunde wird ein Vergleich mit experimentellen Konstanten durchgeführt, mit Hilfe dessen die Genauigkeit der berechneten Konstanten abgeschätzt werden kann. Im Zuge dessen werden auch die alternativen Konzepte der Volumen- und Strukturabhängigkeit des Feldgradienten auf Basis der Gradient-elastischen Tensoren neu bewertet.

Die unter uniaxialer Last durchgeführten Experimente werden anschließend quantitativ im Hinblick auf die Versetzungsdichte, die elastische Verbreiterung und die dabei für die MAX-Phasen relevanten materialphysikalischen Erkenntnisse analysiert. Die in Mischkristallen beobachteten Effekte lassen sich zwar nicht mit Hilfe der Gradient-elastischen Tensoren beschreiben, werden aber gleichwohl im Kontext der Feldgradientenverbreiterung erörtert. Die sich in der Summe ergebenden Möglichkeiten, mit der PAC-Methode interne Spannungen zu messen, werden abschließend bereits etablierten Methoden gegenüber gestellt.

\subsection{Ursache der Dehnungsabhängigkeit des Feldgradienten ${ }^{a}$}

Allein anhand der in den Tabellen 4.1 und 4.2 aufgeführten Werte lässt sich, von Vorzeichen ausgenommen, keine allgemeine Systematik innerhalb der Gradient-elastischen Konstanten erkennen. Es ist nicht klar, warum und wie stark sich der elektrische Feldgradient mit der Dehnung ändert. Dies wird anhand einer Analyse der elektronischen Zustandsdichte im Folgenden erklärt. Solche Analysen im Hinblick auf den EFG sind üblich, um herauszufinden, welche Zustände den EFG hervorrufen [122, 196]. Die Klassifizierung erfolgt hierbei über Energie, Quantenzahl und Art der Zustände.

In Aluminium ist diese Analyse besonders anschaulich, da der EFG hier nur durch pZustände erzeugt wird. Die entsprechende Analyse dieser p-Zustände in der Valenzregion ist in Abbildung 6.1 exemplarisch für eine Dehnung $\varepsilon_{z z}$ gezeigt. Die Dehnung entlang der z-Achse führt zu einer identischen Änderung der Zustandsdichten $n\left(\mathrm{p}_{x}\right)$ und $n\left(\mathrm{p}_{y}\right)$ sowie

${ }^{a}$ Dieses Kapitel basiert im Wesentlichen auf Ergebnissen, die in Referenz [185] veröffentlicht wurden. 
(a)

(b)

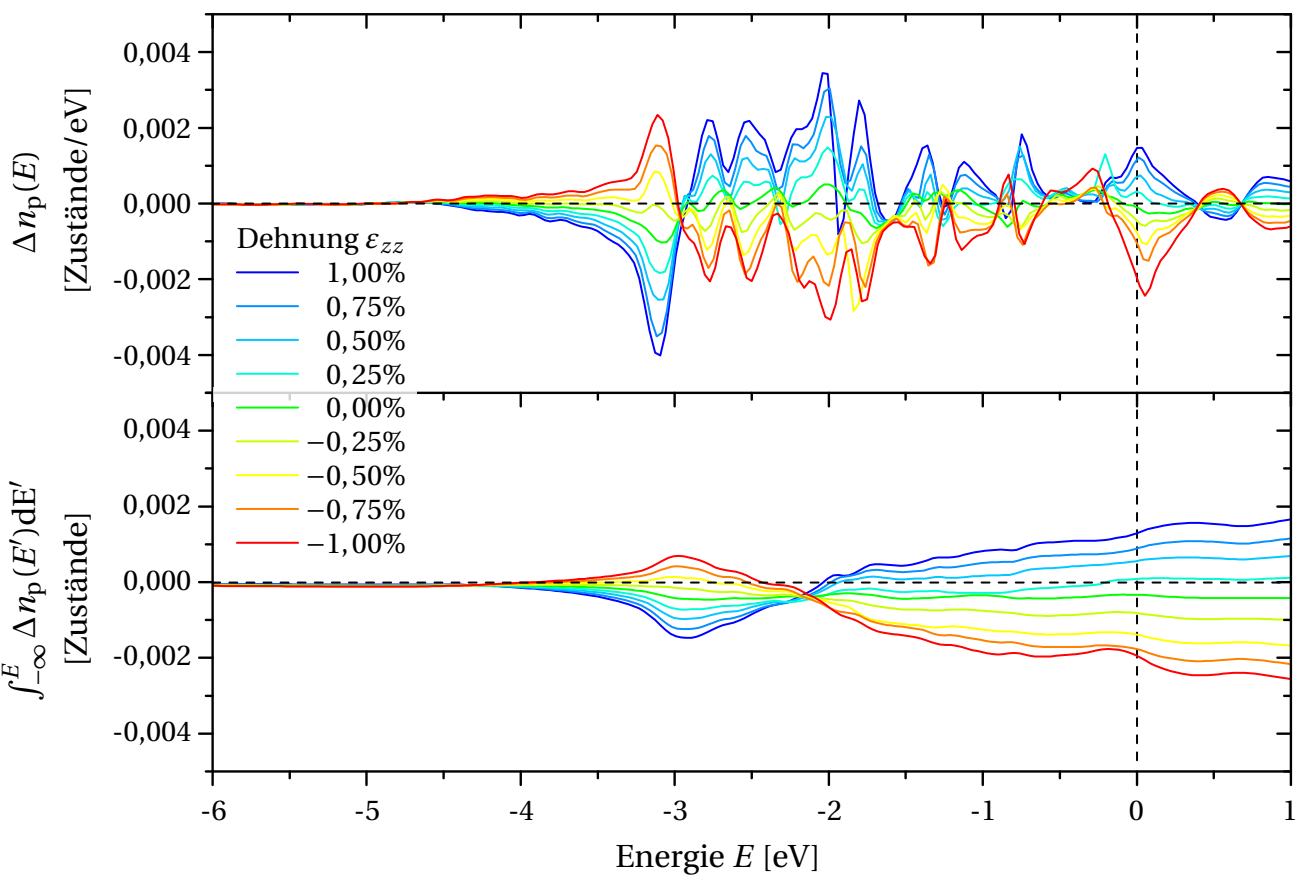

Abbildung 6.1: Asymmetrische Besetzung der p-Zustände $\Delta n_{\mathrm{p}}:=1 / 2\left(n\left(\mathrm{p}_{x}\right)+n\left(\mathrm{p}_{y}\right)\right)-n\left(\mathrm{p}_{z}\right)$ relativ zur Fermienergie in entlang $\varepsilon_{z z}$ gedehntem Aluminium (a). Integral dieser Asymmetrie als Funktion der Energie (b). Die Auswertung dieses Integrals an der Fermienergie ergibt eine zum erzeugten EFG proportionalen Größe. Folglich bestimmen Änderungen dieser integrierten p-Asymmetrie die Gradient-elastischen Konstanten. Negative Werte des Integrals an der Fermienergie entsprechen einer hohen Ladungsdichte im $\mathrm{p}_{z}$-Orbital und einem negativen EFG, positive Werte genau dem Gegenteil. Für verschwindende Dehnungen wird aus Symmetriegründen erwartet, dass die Asymmetrie bei allen Energien null ist. Dies ist nicht der Fall, da die Rechnung in erniedrigter Symmetrie durchgeführt wurde. Es folgt ein nicht vollständig verschwindender EFG bei $\varepsilon_{z z}=0$ (vgl. Achsenabschnitt in Abbildung 4.2).

einer davon verschiedenen Änderung der Dichte $n\left(\mathrm{p}_{z}\right)$, wobei sich sowohl die Lage relativ zur Fermienergie als auch die Form der Teilzustandsdichten ändern. Die daraus resultierende Asymmetrie der Zustandsdichte $\Delta n_{\mathrm{p}}:=1 / 2\left(n\left(\mathrm{p}_{x}\right)+n\left(\mathrm{p}_{y}\right)\right)-n\left(\mathrm{p}_{z}\right)$ ändert sich folglich nicht uniform mit der Dehnung, sondern entwickelt sich für verschiedene Energien unterschiedlich. Da der EFG am Kernort durch die asymmetrische Verteilung aller umgebenden Elektronen bestimmt wird, bildet das Integral über die asymmetrische Zustandsdichte eine dem EFG proportionale Größe. Für eine Dehnung $\varepsilon_{z z}$ ist der EFG somit axialsymmetrisch und entlang der z-Achse orientiert, Stärke und Vorzeichen werden über das Integral der Asymmetrie bestimmt.

Allgemein bedeutet dies, dass Gradient-elastischen Konstanten in starker Abhängigkeit zur individuellen Zustandsdichte stehen. Insbesondere ist es nicht möglich, die Stärke der Gradient-elastischen Konstanten allein mit der Anzahl der Elektronen in Verbindung zu bringen, beispielsweise um einen einfachen Zusammenhang zur Kernladungszahl kubi- 
scher Metalle zu finden, vielmehr muss eine Bilanz gegenläufiger Beiträge aller Zustände berücksichtigt werden.

Im Falle der restlichen kubischen Metalle können der EFG und somit die Gradientelastischen Konstanten, analog zur Analyse in Aluminium, mit der asymmetrischen Besetzungen von p- und d-Zuständen in der Valenzregion in Verbindung gebracht werden. Die in Tantal und Gold auftretenden f-Zustände spielen in beiden Fällen nur eine untergeordnete Rolle. Beiträge zum EFG von außerhalb des kernnahen Bereichs, also von ebenen Wellen in der zwischen den Atomen liegenden Region, sind im Allgemeinen vernachlässigbar klein. Die Relevanz kernnaher p- und d-Zustände für den Ursprung des dehnungsinduzierten EFGs in kubischen Metallen steht somit mit dem Ursprung des intrinsischen EFGs in hexagonalen Metallen im Einklang [122].

Das Verhältnis zwischen Beiträgen aus p- und d-Zuständen variiert zwischen den Metallen und zwischen den unterschiedlichen Dehnungen $\varepsilon_{z z}$ und $\gamma_{y z}$, wird aber üblicherweise von den p-Zuständen dominiert. Die Dominanz der p-Zustände kann, in Analogie zu den hexagonalen Metallen [122], mit der radialen Verteilung der anisotropen Ladungsdichte begründet werden. So weisen p-Zustände eine höhere Ladungsdichte in Kernnähe auf, womit diese auch stärker zum EFG beitragen. Eine Ausnahme von dieser Regel bildet die Dehnung $\varepsilon_{z z}$ in Kupfer, bei der der größte Beitrag zum EFG von den d-Zuständen verursacht wird, insgesamt aber eine vergleichsweise geringe Änderung des EFGs auftritt. Hier treten neben den Beiträgen der 4p-Zustände nahe der Fermienergie noch Beiträge von tiefer liegenden, vollbesetzten 3p-Semikernzuständen entgegengesetzten Vorzeichens auf, die den Beitrag der 4p-Zustände nahezu vollständig aufwiegen. Diese auf den ersten Blick überraschenden Beiträge ${ }^{a}$ treten nicht nur in Kupfer sondern auch in Aluminium und Gold auf, wobei der entsprechende Semikernzustand bei einer Energie von 50-60 eV unterhalb der Fermienergie liegt. Ein ähnliches Verhalten wurde schon für seltene Erden beschrieben, in denen vollbesetzte $5 p$-Schalen durch f-Elektronen in der Atomhülle oder durch eine nichtkubische Umgebung asymmetrisch verzerrt werden können [123]. Es ist anzunehmen, dass der gleiche Effekt auch für die vollbesetzten Semikernzustände in den hier beschriebenen kubischen Metallen auftritt, also die asymmetrisch besetzten Zustände nahe der Fermienergie eine anisotrope Besetzung der Semikernzustände induzieren.

Der überall vorhandene Beitrag von Zuständen nahe der Fermienergie bestätigt frühere Rechnungen zu Gradient-elastischen Konstanten insofern als ein starker Beitrag der Leitungselektronen zum EFG vermutet wurde (siehe Referenz [197] und die darin enthaltenen Zitate). Diese auf dem Versagen des Punktladungsmodells basierende Vermutung wird durch die hier gezeigte Analyse auf direktem Wege nachgewiesen.

Für die hexagonalen Systeme werden, genauso wie in den gedehnten kubischen Metallen, die intrinsisch vorhanden Feldgradienten maßgeblich durch eine asymmetrische Besetzung der p- und d-Zustände verursacht [46, 121, 122]. Beiträge von außerhalb des kernnahen Bereichs sind auch hier vernachlässigbar klein. Folglich werden die Gradientelastischen Konstanten in diesen Systemen durch eine dehnungsinduzierte Änderung der asymmetrischen Besetzung bestimmt. Das Verhältnis zwischen Beiträgen aus p- und dZuständen variiert wie in den kubischen Metallen zwischen den Materialsystemen und

${ }^{a}$ In freien Atomen bilden vollbesetzte Schalen eine isotrope Ladungsdichte, tragen also nicht zum EFG bei. 


\section{Kapitel 6 Diskussion}

den unterschiedlichen Dehnungen, wird im Allgemeinen aber durch die Beitrag der pZustände dominiert.

Die nicht-lineare Änderung des EFGs an der Cadmiumsonde in Titan kann vollständig mit einer nicht-linearen Änderung der asymmetrischen Besetzung der p-Zustände in Verbindung gebracht werden. Der ungefähr gleich große Anteil der d-Zustände an der Änderung des EFGs verhält sich hingegen vollständig linear. Eine Untersuchung der integrierten p-Asymmetrie an der Fermienergie ergibt keine Hinweise auf die Frage, warum sich die p-Asymmetrie lediglich an der Cadmiumsonde in Titan so eigentümlich verhält. Gleichermaßen bieten sich durch eine solche Analyse keine fundamentaleren Einsichten in das von den anderen MAX-Phasen abweichende Verhalten des EFGs an der Cadmiumsonde in $\mathrm{Ti}_{2} \mathrm{AlC}$. Um zu klären, ob diese Besonderheiten Artefakte der Rechnung oder reale Eigenschaften der Materialsysteme sind, wäre eine experimentelle Untersuchung an Einkristallen nötig.

Eine Vorhersage der Gradient-elastischen Konstanten, beispielsweise durch die Steigung der integrierten Asymmetrie an der Fermienergie, ist im Allgemeinen nicht möglich. Das einfachste Gegenbeispiel bieten hierbei kubische Systeme, deren asymmetrische Besetzung aus Symmetriegründen für alle Energien null ist. Es ist deshalb für die Bestimmung der Gradient-elastischen Konstanten unumgänglich, die explizite Änderung der Zustandsdichte mit der Dehnung zu bestimmen.

\subsection{Genauigkeit der ermittelten Gradient-elastischen Konstanten ${ }^{a}$}

Ein direkter Vergleich der hier berechneten Gradient-elastischen Konstanten mit experimentellen Werten ist nur bedingt möglich, da allein für die kubischen Metalle entsprechende Literaturwerte vorliegen. In anderen Materialsystemen können lediglich von den Gradient-elastischen Konstanten abgeleitete Größen, wie die relative Änderung des EFGs unter isostatischem Druck oder unter uniaxialer Spannung entlang dedizierter Kristallachsen, als Vergleichsgröße herangezogen werden. In beiden Fällen werden im Folgenden anhand der Abweichung zum Literaturwert die Fehler der berechneten Konstanten abgeschätzt. Der Einfluss von Temperatureffekten und anderer Fehlerquellen wird dabei zunächst vernachlässigt. Aus dieser Abschätzung in experimentell zugänglichen Systemen wird auf die zu erwartende Genauigkeit in vergleichbaren, experimentell nicht zugänglichen Systemen geschlossen.

\subsubsection{Direkter Vergleich zu experimentellen Werten}

Der Vergleich zwischen berechneten und bei Raumtemperatur gemessenen Gradientelastischen Konstanten $S_{m n}$ ist in Tabelle 6.1 gezeigt. Die experimentellen Werte wurden, wo möglich, mit aktuellen Quadrupolmomenten [198] neu bewertet. Es ist im Hinblick auf die Genauigkeit der experimentellen Werte davon auszugehen, dass Messungen mit einer akustischen Anregung des Kernresonanzsignals (engl. nuclear acustic resonance, NAR)

${ }^{a}$ Dieses Kapitel basiert im Wesentlichen auf Ergebnissen, die in Referenz [185] veröffentlicht wurden. 
Tabelle 6.1: Vergleich berechneter und experimenteller Gradient-elastischer Konstanten $S_{m n}$.

\begin{tabular}{cccccc}
\hline Wirtsgitter & \multicolumn{2}{c}{$S_{11}\left[10^{21} \mathrm{~V} / \mathrm{m}^{2}\right]$} & \multicolumn{2}{c}{$S_{44}\left[10^{21} \mathrm{~V} / \mathrm{m}^{2}\right]$} & Methode \\
& ber. & exp. & ber. & exp. & \\
\hline $\mathrm{Al}$ & $5,46(5)$ & 5,5 & $-8,02(1)$ & $-5,6$ & NMR [82] \\
& & $\pm 4,7^{a}$ & & $\mp 8,4^{a}$ & NAR [81] \\
$\mathrm{Au}$ & $13,28(9)$ & $\pm 27(3)$ & $-49,42(1)$ & $\mp 39(2)$ & NAR [87] \\
$\mathrm{Mo}$ & $49,2(3)$ & $\pm 10(3)^{a}$ & $-8,37(1)$ & $\mp 3,1(7)^{a}$ & NAR [85] \\
$\mathrm{Nb}$ & $15,4(2)$ & $\pm 15(3)^{a}$ & $-37,66(5)$ & $\mp 42(7)^{a}$ & NAR [84] \\
$\mathrm{Ta}$ & $68,2(1)$ & $\pm 65(6)^{a}$ & $-77,5(1)$ & $\mp 74(4)^{a}$ & NAR [86] \\
& & $\pm 92(10)^{a}$ & & $\mp 102(11)^{a}$ & NAR [83] \\
\hline
\end{tabular}

${ }^{a}$ Mit Quadrupolmomenten aus Ref. [198].

den statischen Messungen unter elastischer Spannung (engl. nuclear magnetic resonan$c e$, NMR) überlegen sind. Dessen ungeachtet weichen die experimentellen Werte unterschiedlicher Gruppen trotz gleicher Messmethode um 20-50\% voneinander ab, womit ein Anhaltspunkt für die Genauigkeit der experimentellen Bestimmung gegeben ist.

Eine vergleichsweise gute Übereinstimmung zwischen Rechnung und Experiment liegt für Aluminium, Niob und Tantal vor. In Gold und Molybdän weichen die Rechnungen um $100 \%$ bzw. $370 \%$ vom Experiment ab. Es ist nicht klar, warum sich die Werte lediglich in Molybdän so stark unterscheiden. Der Literaturwert von Kupfer wurde zusammen mit einem Wert für Aluminium [79] veröffentlicht, der im Nachhinein entkräftet wurde [82]. Da nicht klar ist, was in der vorhergehenden Bestimmung misslang, wird auf einen Vergleich der Werte für Kupfer verzichtet. Im Schnitt ergibt sich so für die kubischen Metalle eine Abweichung zwischen Experiment und Rechnung die, Molybdän ausgenommen, um 25\% liegt.

\subsubsection{Vergleich mit abgeleiteten Größen}

Im Falle von Systemen mit intrinsischem EFG wird üblicherweise die relative Änderung der Quadrupolkopplungskonstante $v_{Q}$ oder die Änderung des Asymmetrieparameters $\eta$ unter einer isostatischen oder uniaxialen Spannung betrachtet [88, 89, 99, 100, 124]. Beide experimentellen Größen lassen sich aus den hier berechneten Gradient-elastischen Konstanten vorhersagen und somit zum Testen der Rechnung nutzen. Im Folgenden wird isostatischer Druck mit $p$ gekennzeichnet. Der zugehörige Spannungstensor lautet

$$
\sigma_{i j}=\left(\begin{array}{ccc}
-p & 0 & 0 \\
0 & -p & 0 \\
0 & 0 & -p
\end{array}\right) .
$$

Im Falle uniaxialer Belastungen $\sigma_{a}, \sigma_{m}$ und $\sigma_{c}$ entlang der dedizierten Kristallachsen $a$, $m$ und $c$ werden die entsprechenden Elemente des Spannungstensors, analog zum Druck, mit dem negativen Betrag gebildet. Die betrachteten relativen Änderungen werden gemäß

$$
\frac{1}{V_{z z}^{0}} \frac{\mathrm{d} V_{z z}}{\mathrm{~d} p}=\frac{1}{v_{Q}^{0}} \frac{\mathrm{d} v_{Q}}{\mathrm{~d} p}:=\frac{\mathrm{d} \ln v_{Q}}{\mathrm{~d} p}
$$


Tabelle 6.2: Vergleich zwischen berechneten und experimentellen relativen Änderungen der größten EFG-Tensorkomponente unter kompressiven Spannungen. Alle Größen sind in $\mathrm{GPa}^{-1}$ angegeben.

\begin{tabular}{ccccc}
\hline Wirtsgitter & Messgröße & berechnet & experimentell & Methode \\
\hline \multirow{2}{*}{$\mathrm{dd}$} & $\mathrm{d} \ln v_{Q} / \mathrm{d} \sigma_{c}$ & $-0,227(1)$ & $-0,28(12)$ & PAC [89] \\
& $\mathrm{d} \ln v_{Q} / \mathrm{d} p$ & $-0,053(2)$ & $-0,036(3)$ & PAC [100] \\
& & & $-0,043(3)$ & PAC [99] \\
Zn & $\mathrm{d} \ln v_{Q} / \mathrm{d} p$ & $-0,071(6)$ & $-0,056(4)$ & PAC [124] \\
ZnO & $\mathrm{d} \ln v_{Q} / \mathrm{d} \sigma_{c}$ & $0,223(5)$ & $0,196(4)$ & PAC [88] \\
& $\mathrm{d} \ln v_{Q} / \mathrm{d} \sigma_{a}$ & $-0,112(4)$ & $-0,245(5)$ & PAC [88] \\
& $\mathrm{d} \ln v_{Q} / \mathrm{d} \sigma_{m}$ & $-0,112(4)$ & $-0,245(5)$ & PAC [88] \\
\hline
\end{tabular}

mit der ursprünglichen Quadrupolkopplungskonstante $v_{Q}^{0}$ bzw. dem ursprünglichen EFG $V_{z z}^{0}$ gebildet. Als Bezugsgröße für die Berechnung der relativen Änderung des intrinsischen EFGs wird der experimentell ermittelte Wert bei $T=0 \mathrm{~K}$ verwendet (siehe Tabelle 4.2). Dadurch wird implizit angenommen, dass der jeweils zugrundeliegende Unterschied zwischen experimentellem und berechnetem EFG keinen Einfluss auf die Gradientelastischen Konstanten hat, also lediglich eine konstante Verschiebung bildet.

Solange das Hauptachsensystem des Feldgradienten unter Last gleich bleibt, kann die relative Änderung unmittelbar durch Spannungstensor, Gradient-elastischen Tensor $C_{m n}$ und Gleichung 2.5 ausgedrückt werden. Dies trifft auf alle Spannungszustände in Systemen mit Punktgruppe $D_{6 h}$ und $D_{3 h}$ zu, die im Hauptachsensystem des ursprünglichen EFGs lediglich aus Normalspannungen bestehen. In diesen Fällen verhalten sich Spannungen in der Basalebene isotrop, eine Unterscheidung zwischen der $a$ - und der $m$-Achse ist damit nicht nötig. In Systemen mit Punktgruppe $C_{3 v}$, also beispielsweise der Cadmiumsonde in Zinkoxid, bleibt das Hauptachsensystem nur für Normalspannungen mit $\sigma_{x x}=\sigma_{y y}$, das heißt isostatischem Druck oder Spannungen entlang der c-Achse, erhalten. Für Spannungen in der Basalebene muss der EFG-Tensor in das neue Hauptachsensystem transformiert werden, bevor die entsprechenden Änderungen von $v_{Q}$ und $\eta$ bestimmt werden können. Hieraus resultiert für große Spannungen eine nicht-lineare Änderung dieser Größen (vgl. Titan in Kapitel 4.2.4). Ferner müssen aufgrund fehlender Isotropie in der Basalebene Spannungen entlang der $a$ - und der $m$-Achse unterschieden werden. Ein Vergleich zwischen den bei $T=0 \mathrm{~K}$ berechneten und den bei Raumtemperatur gemessenen Abhängigkeiten der Quadrupolkopplungskonstante in Cadmium, Zink und Zinkoxid ist in Tabelle 6.2 gegeben.

Für Cadmium und Zink müssen Fehler von 5\% bis $10 \%$ für die unabhängigen Komponenten von $S_{m n}$ angenommen werden, um mit den experimentellen Werten vereinbar zu sein. Dieser Fehler wirkt zunächst sehr klein, genügt aber der beobachteten Abweichung, da die Messgrößen aus der Summe dreier unabhängiger Konstanten unterschiedlichen Vorzeichens gebildet werden.

Für Zinkoxid muss ein Fehler von 15\% angenommen werden, um mit dem experimentellen Wert von $\mathrm{d} \ln v_{Q} / \mathrm{d} \sigma_{c}$ in Einklang zu stehen. Die Abweichungen für Spannungen in der Basalebene sind erheblich höher, müssen aber unter Vorbehalt betrachtet werden. Wie 
schon in Kapitel 3.1 angedeutet, führt die in jeder Messung auftretende Verbreiterung der EFG-Tensorkomponenten zu einem systematischen Fehler in der Bestimmung der Quadrupolkopplungskonstante [164]. Dieser Fehler hängt vom Asymmetrieparameter ab und führt bei $\eta=0$ zu einer maximalen Überschätzung von $v_{Q}$. Da nun aber gerade der Asymmetrieparameter bei Spannungen in der Basalebene ausgehend von null ansteigt, ist davon auszugehen, dass die Beträge der Messgrößen $\mathrm{d} \ln v_{Q} / \mathrm{d} \sigma_{a}$ und $\mathrm{d} \ln v_{Q} / \mathrm{d} \sigma_{m}$ experimentell überschätzt wurden. Unabhängig von der Abweichung zwischen Experiment und Rechnung ist auch der Unterschied zwischen verschiedenen Orientierungen in der Basalebene von Interesse. Hierbei fällt auf, dass sich sowohl in der Rechnung als auch im Experiment $v_{Q}$ für beide Orientierungen nahezu gleich verhält.

Dieses Verhalten wird auch für die Rechnung des Asymmetrieparameters beobachtet, dessen Spannungsabhängigkeit entlang der $a$ - bzw. $m$-Achse bis zu 1 GPa nahezu identisch verläuft. Für eine dem Messbereich aus Referenz [121] entsprechende Spannung von $0.3 \mathrm{GPa}$ errechnet sich ein Wert von $\mathrm{d} \eta / \mathrm{d} \sigma_{a}=\mathrm{d} \eta / \mathrm{d} \sigma_{m}=0,44(1) \mathrm{GPa}^{-1}$. Dieser Wert ist zwar mit dem experimentell beobachteten Wert $\mathrm{d} \eta / \mathrm{d} \sigma_{a}=0,5(1) \mathrm{GPa}^{-1}$ vereinbar, nicht aber mit $\mathrm{d} \eta / \mathrm{d} \sigma_{m}=1,1(2) \mathrm{GPa}^{-1}$. Um diesen experimentell beobachteten Unterschied der beiden Orientierungen mit der hier verwendeten Theorie beschreiben zu können, würde ein derart großer Wert der Konstante $S_{41}$ benötigt werden, dass dadurch zwangsläufig auch ein signifikanter Unterschied im Verhalten von $v_{Q}$ experimentell sichtbar sein müsste. Dies ist aber, wie oben gesehen, nicht der Fall. Somit stößt hier entweder die linear-elastische Theorie des Feldgradienten an ihre Grenzen oder aber die Messung des Asymmetrieparameters ist zu ungenau.

\subsubsection{Relevante Fehlerquellen}

Der Mittelwert aller bisher ermittelten Abweichung suggeriert eine Genauigkeit der berechneten Konstanten von über 30\%. Eine Übersicht der Korrelation zwischen Rechnung und Experiment der hier gezeigten Messgrößen ist in Abbildung 6.2 gegeben. Die Erwartung, dass die Genauigkeit mit zunehmender Komplexität der Systeme zurückgeht, kann anhand der untersuchten Systeme nicht bestätigt werden; lediglich die Streuung der berechneten Datenpunkte, aus denen die Konstanten ermittelt werden, nimmt zu. Bei all den bisher angestellten Vergleichen wurden einige offensichtliche Fehlerquellen ignoriert, die im Folgenden angesprochen werden. Die trotz dieser unberücksichtigten Fehler relativ hohe Genauigkeit spricht für die Beständigkeit der hier vorgestellten Methode zur Bestimmung der Gradient-elastischen Konstanten.

Ein erster, schon angesprochener Fehler betrifft die Temperatur. Alle Vergleichsmessungen wurden bei Raumtemperatur durchgeführt, die berechneten Konstanten sind jedoch lediglich bei $T=0$ K gültig. Der dadurch eingebrachte Fehler kann anhand der Temperaturabhängigkeit des intrinsischen EFGs in hexagonalen Systemen mit $2 \%$ bis $20 \%$ abgeschätzt werden [47, 186-189].

Alle Systeme, in denen eine Superzelle zur Berechnung verwendet wird, erfahren durch deren limitierte Größe einen systematischen Fehler. Im Idealfall entspräche die Größer der Superzelle dem mittleren Abstand der experimentell eingebrachten Sondenatome. Zur weiteren Berechnung müssten zudem die elastischen Konstanten eben dieser Superzelle 

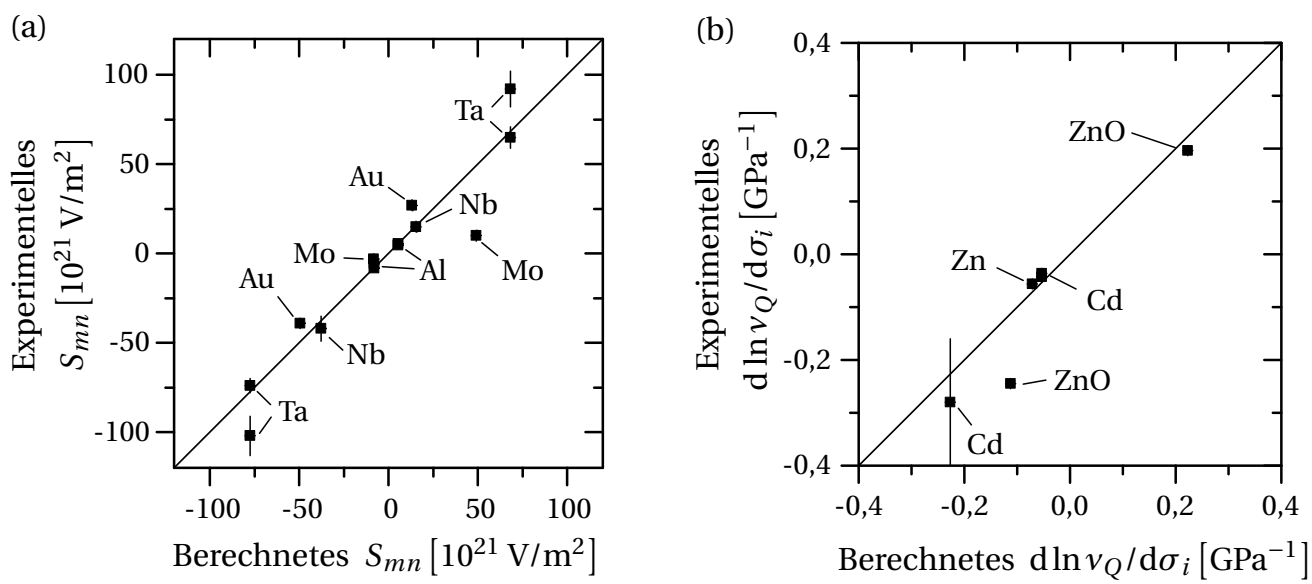

Abbildung 6.2: Korrelation zwischen den berechneten und gemessenen Gradient-elastischen Konstanten $S_{m n}$ aus Tabelle 6.1 (a) sowie den relativen Spannungsabhängigkeiten der größten EFG-Tensorkomponente aus Tabelle 6.2 (b).

verwendet werden. Beides führt zu extrem aufwändigen Rechnungen. Ansatzweise könnte diesem Fehler Rechnung getragen werden, indem zumindest die elastischen Konstanten der tatsächlich verwendeten Superzelle berechnet werden, jedoch würde auch dies bereits zu einem erheblich erhöhten Rechenaufwand führen. Somit können die hier präsentierten Rechnungen unter Ausnutzung der elastischen Konstanten des Volumenmaterials als brauchbarer Kompromiss zwischen Rechenzeit und Genauigkeit angesehen werden, da beim Übergang hin zu komplexen Systemen keine signifikant schlechteren Ergebnisse erzielt werden.

Die hierbei und in einfacheren Systemen verwendeten elastischen Konstanten sind ihrerseits selbstverständlich auch nicht exakt. So können elastische Konstanten experimentell bis auf wenige Prozent genau bestimmt werden [55-57, 59-63]. Berechnete Konstanten, wie sie zum Beispiel im Falle der MAX-Phasen aus Ermangelung experimenteller Werte verwendet werden müssen, weisen dagegen vermutlich eine Genauigkeit von ungefähr $10 \%$ auf [40].

Zuletzt ist auch die Genauigkeit der experimentellen Vergleichsgrößen selbst bisher unberücksichtigt geblieben. Wie anhand von Gold und Tantal deutlich wird, ist hierbei mit einer Ungenauigkeit von einigen zehn Prozent zu rechnen [81-83, 86]. Diese Ungenauigkeit wird durch den Fehler des Quadrupolmomentes zusätzlich verstärkt, da dieses Moment bei der Umrechnung der Quadrupolfrequenzen in Feldgradienten eine zentrale Rolle einnimmt. Abhängig vom Isotop schlägt dieser Fehler mit ungefähr $5 \%$ zu buche [120, 198].

Im Vergleich zu den Rechnungen von Zwanziger et. al. in Galliumphosphid [90] werden leicht bessere Ergebnisse erzielt. Der Vergleich zu den experimentellen Werten [78] zeigt hier eine Abweichung von $60 \%^{a}$. Da die Konstanten nur für ein Materialsystem berechnet wurden, ist kein systematischer Vergleich zu den hier gezeigten Rechnungen mög-

\footnotetext{
${ }^{a}$ Zwanziger et.al. [90] berechneten in Galliumphosphid $S_{11}$ und $S_{44} \mathrm{zu} 18 \cdot 10^{21} \mathrm{~V} / \mathrm{m}^{2}$ bzw. $-20 \cdot 10^{21} \mathrm{~V} / \mathrm{m}^{2}$. Die entsprechenden experimentellen Werte lauten mit dem aktuellen Quadrupolmoment der Galliumsonde $\pm 33,2 \cdot 10^{21} \mathrm{~V} / \mathrm{m}^{2}$ bzw. $\mp 28,6 \cdot 10^{21} \mathrm{~V} / \mathrm{m}^{2}[78,199]$.
} 
lich. Im Allgemeinen wird aber ein besseres Abschneiden der hier verwendeten LAPW- bzw. APW+lo-Methode erwartet, weil die in Referenz [90] verwendete Pseudopotentialmethode kernnahe Elektronendichten nur ungenügend beschreibt (siehe Kapitel 2.3).

\subsection{Volumen- und Strukturabhängigkeit des Feldgradienten ${ }^{a}$}

Das neben dem Gradient-elastischen Tensor entwickelte Konzept, Änderungen des EFGs linear an eine Änderung des $c / a$-Verhältnisses oder des Volumens zu koppeln führte zu der Schlussfolgerung, dass der EFG in hexagonalen Metallen ungefähr bei idealdichtester Packung $c / a=1,633$ verschwindet und sich überproportional stark mit dem Volumen ändert [89]. Die Bestimmung der Volumenabhängigkeit setzte hierbei voraus, dass der Feldgradient exakt bei idealdichtester Packung verschwindet. Diese wechselseitige Abhängigkeit kann mit dem hier bestimmten, vollständigen Gradient-elastischen Tensor umgangen werden. Es ist somit zum ersten Mal möglich, Volumen- und Strukturabhängigkeit des Feldgradienten direkt zu bestimmen.

Die Strukturabhängigkeit beschreibt die relative Änderungen des Feldgradienten mit der relativen Änderung des $c / a$-Verhältnisses bei konstantem Volumen. Der zugehörige Dehnungstensor, der die Abweichung vom ursprünglichen Verhältnis $c_{0} / a_{0}$ beschreibt, lautet

$$
\varepsilon_{i j}=\left(\begin{array}{ccc}
\alpha & 0 & 0 \\
0 & \alpha & 0 \\
0 & 0 & \frac{1-(1+\alpha)^{2}}{(1+\alpha)^{2}}
\end{array}\right), \quad \text { mit } \alpha=\sqrt[3]{\frac{c_{0} / a_{0}}{c / a}}-1
$$

Entsprechend der in Gleichung 6.2 eingeführten Definition einer relativen Änderung des EFGs ergibt sich die relative Strukturabhängigkeit durch eine lineare Entwicklung nach $c / a$, also für kleine Änderungen, zu

$$
\left(\frac{\partial \ln v_{Q}}{\partial \ln c / a}\right)_{V}=\frac{1}{3 V_{z z}^{0}}\left(-S_{31}-S_{32}+2 S_{33}\right) .
$$

Um herauszufinden, bei welchem $c / a$-Verhältnis der EFG verschwindet, muss diese Abhängigkeit für große Änderungen, also fernab vom linear-elastischen Bereich, untersucht werden. Es ist somit zunächst nicht klar, wie aussagekräftig eine solche Untersuchung ist. Diese Problematik sprach auch schon Butz [89] in seiner experimentellen Untersuchung der Strukturabhängigkeit an, in der eine Extrapolation experimenteller Daten zur Identifikation des entsprechenden $c / a$-Verhältnisses nötig war.

Im Rahmen der hier präsentierten Untersuchung ergeben sich zwei unterschiedliche Herangehensweisen, mit dieser Extrapolation umzugehen. Einerseits könnte direkt der Dehnungstensor aus Gleichung 6.3 in Gleichung 2.5 eingesetzt werden, um so die Strukturabhängigkeit im Rahmen der linear-elastischen Theorie exakt zu berechnen, andererseits könnte die lineare Entwicklung nach $c / a$ aus Gleichung 6.4 extrapoliert werden. Der erste Weg sagt eine nicht-lineare Änderung des EFGs mit dem $c / a$-Verhältnis voraus, der zweite eine lineare; für kleine Änderungen stimmen beide Verläufe überein.

${ }^{a}$ Dieses Kapitel basiert im Wesentlichen auf Ergebnissen, die in Referenz [185] veröffentlicht wurden. 


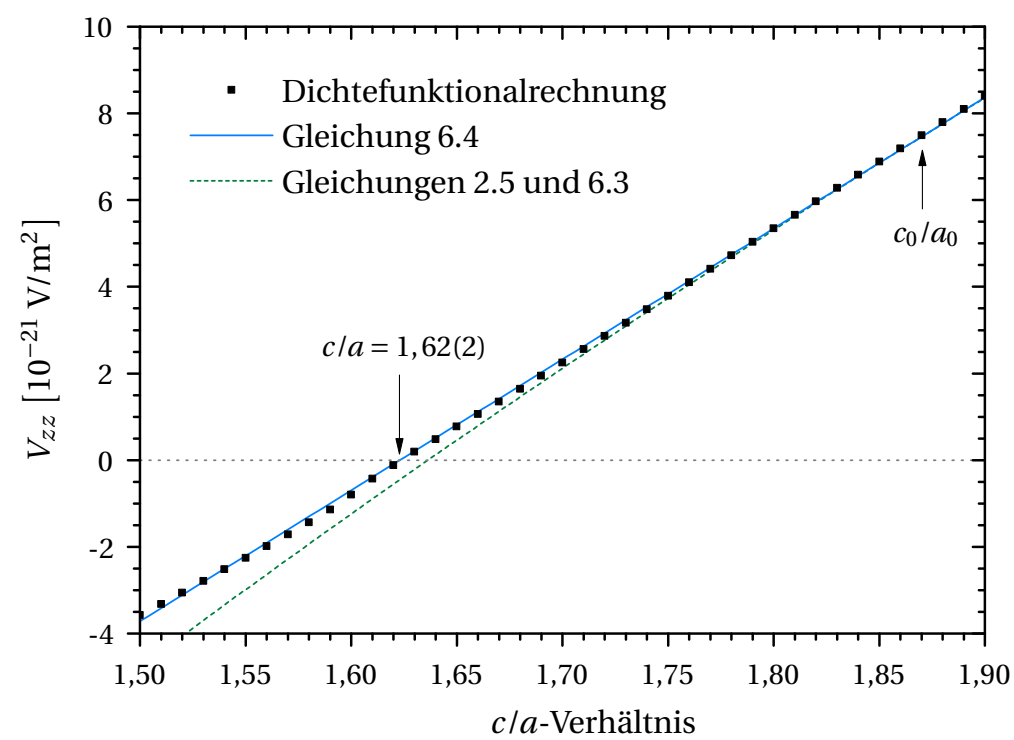

Abbildung 6.3: Der elektrischen Feldgradient in Cd als Funktion des $c / a$-Verhältnisses bei konstantem Volumen. Die berechneten Werte zeigen über weite Bereiche einen linearen Zusammenhang mit der von der linear-elastischen Theorie für kleine Änderungen vorhergesagten Steigung (Gleichung 6.4). Dies verdeutlicht die auf kleine Dehnungen limitierte Gültigkeit des allgemeinen Ansatzes der linear-elastischen Theorie (Gleichungen 2.5 und 6.3). Der EFG verschwindet bei $c / a=1,62(2)$, also ungefähr bei $c / a=1,633$, dem Verhältnis idealdichtester Packung.

Welcher Weg besser die Realität beschreibt, kann mittels einer direkten Berechnung des Feldgradienten bei vorgegebenem $c / a$-Verhältnis erfolgen. Es zeigt sich über weite Bereiche ein linearer Zusammenhang, der exakt mit der Vorhersage aus Gleichung 6.4 übereinstimmt (siehe Abbildung 6.3). Dies verdeutlicht die auf kleine Dehnungen limitierte Gültigkeit des allgemeinen Ansatzes der linear-elastischen Theorie des Feldgradienten; für eine Beschreibung bei großen Dehnungen müssten höhere Ordnungen berücksichtigt werden. Die Tatsache, dass gerade die lineare Entwicklung nach $c / a$ den EFG korrekt beschreibt, zeigt die zentrale Bedeutung des $c / a$-Verhältnisses für den EFG.

Das $c / a$-Verhältnis, bei dem der EFG verschwindet kann nun direkt für die hexagonalen Metalle ausgewertet werden ${ }^{a}$. Für Cadmium und Zink wird ein Verschwinden des EFGs an der Cadmiumsonde bei einem Verhältnis von 1,62(2) bzw. 1,68(4) vorhergesagt, in Übereinstimmung mit dem Verhältnis von 1,633 bei idealdichtester Packung. Der Wert für Titan ist mit 2,0(9) aufgrund der großen Fehler der beteiligten Gradient-elastischen Konstanten nicht sonderlich informativ.

Die Volumenabhängigkeit beschreibt die relative Änderung des EFGs mit der relativen Änderung des Volumens bei konstantem $c / a$-Verhältnis. Der zugehörige Dehnungstensor,

\footnotetext{
${ }^{a}$ In binären und ternären Systemen ist eine solche Auswertung aufgrund der verschiedenen auftretenden Elemente nicht sinnvoll.
} 
der die Abweichung vom ursprünglichen Volumen $V_{0}$ beschreibt, lautet

$$
\varepsilon_{i j}=\left(\begin{array}{ccc}
\beta & 0 & 0 \\
0 & \beta & 0 \\
0 & 0 & \beta
\end{array}\right), \quad \text { mit } \beta=\sqrt[3]{\frac{V}{V_{0}}}-1 .
$$

Die relative Volumenabhängigkeit des EFGs lautet somit für kleine Änderungen

$$
\left(\frac{\partial \ln v_{Q}}{\partial \ln V}\right)_{c / a}=\frac{1}{3 V_{z z}^{0}}\left(S_{31}+S_{32}+S_{33}\right) .
$$

Die so ermittelte Volumenabhängigkeit des EFGs an der Cadmiumsonde beträgt für Zink $-1,7(2)$, was in Übereinstimmung mit dem experimentell abgeleiteten Wert $-1,9(8)$ [89] steht. Im Falle von Cadmium wird eine Volumenabhängigkeit von -1,76(6) vorhergesagt. Aus Experimenten wurden Werte von -3,7(4) und -4, 1(5) abgeleitet [89]. Diese Werte stehen selbst dann nicht im Einklang mit dem hier berechneten Wert, wenn ein Fehler der Gradient-elastischen Konstanten von 10\% angenommen wird (siehe Diskussion in Kapitel 6.2), womit sich eine Volumenabhängigkeit von -1,8(4) ergeben würde.

Abweichungen werden an dieser Stelle erwartet, da die vom Experiment abgeleiteten Werte unter der Annahme eines exakt bei idealdichtester Packung verschwindenden EFGs ermittelt wurden. Diese Annahme hat einen dramatischen Einfluss auf das erhaltene Ergebnis. So verringert sich die Volumenabhängigkeit um 50\%, wenn in der Berechnung statt dieser Annahme die aus den Gradient-elastischen Konstanten erhaltene Strukturabhängigkeit verwendet wird. Des Weiteren wurden für die dortige Berechnung druckabhängige Gitterkonstanten verwendet, die im Vergleich zu einer Berechnung mit (genaueren) elastischen Konstanten zu einer zusätzlichen Überschätzung von $20 \%$ führen. Die hier ermittelten Werte sind unabhängig von elastischen Konstanten oder der Strukturabhängigkeit des EFGs, bieten also - Temperatureffekte ausgenommen - den direkteren Zugang zur Volumenabhängigkeit.

Die Volumenabhängigkeiten der MAX-Phasen liegen zwischen -0,9(2) für $\mathrm{Ti}_{2} \mathrm{AlC}$ und -1,61(6) für $\mathrm{Ti}_{2} \mathrm{InC}$, der EFG ändert sich in den MAX-Phasen also weniger stark mit dem Volumen als in den hexagonalen Metallen.

Die obige Diskussion bestätigt somit unmittelbar frühere Untersuchungen [89], die zum Bild eines in der Nähe idealdichtester Packung verschwindenden EFGs führten, der sich überproportional stark mit dem Volumen verändert. Übertragen auf die Zustandsdichten bedeutet dies, dass es in hexagonalen Metallen eine Konfiguration gibt, in der sich die Asymmetrien innerhalb der Unterzustandsdichten gerade aufheben. Änderungen des Volumens haben einen starken Einfluss auf diese Asymmetrie, die Unterzustandsdichten ändern sich also unabhängig voneinander: Lediglich für eine isotrope Änderung der Ladungsdichte würde aufgrund der $1 / r^{3}$-Abhängigkeit des Feldgradienten eine relative Volumenabhängigkeit von exakt -1 folgen.

\subsection{Linear-elastische Störungen des Feldgradienten}

Die Erweiterung von uniformen Dehnungen eindeutiger Orientierung hin zu inhomogenen Dehnungen verschiedener Orientierungen führt zu einer Verteilung des Feldgradien- 


\section{Kapitel 6 Diskussion}

ten, die unter Ausnutzung der Gradient-elastischen Konstanten eindeutig mit der Ursache der jeweiligen Störung in Verbindung gebracht werden kann. Durch die Verknüpfung der Charakteristika verschiedener Störungen (siehe Kapitel 4.2) mit den in Kapitel 5 beobachteten Phänomenen lassen sich nun Aussagen über damit einhergehende materialphysikalische Vorgänge treffen.

\subsubsection{Auswirkungen der Deformation ${ }^{a}$}

Die Auswirkungen der Deformation auf die Feldgradientenverteilung zeigen sich in einem Anteil, der reversibel unter Last auftritt, und einem Anteil, der irreversibel mit der permanenten Deformation einhergeht. Im PAC-Experiment wird dies als reversibler bzw. irreversibler Anstieg der Dämpfung $\delta$ der Störfunktion sowie einer reversiblen Änderung der Quadrupolkopplungskonstante sichtbar (siehe Kapitel 5.1).

Aus den Experimenten ergibt sich somit folgendes Bild: Die anfänglich auftretende Dämpfung $\delta_{0}$ wird durch die nach der Implantation vorhandene Punkt- und Versetzungsdichte bestimmt. Durch die Deformation eingebrachte Versetzungen und etwaige damit einhergehende Punktdefekte sind für den irreversiblen Anstieg verantwortlich. Risse und Delamination spielen hierbei vermutlich nur eine untergeordnete Rolle, da lediglich neue Oberflächen erzeugt werden. Die durch die uniaxiale Last hervorgerufene reversible Änderung der Quadrupolkopplungskonstante und der Dämpfung gehen auf die elastische Verbreiterung zurück. Ergänzend dazu sind Beiträge zur Dämpfung denkbar, die von einer unter Last erhöhten Versetzungsdichte stammen. Punktdefekte, die nur unter Last existieren, können hingegen ausgeschlossen werden.

Einflüsse von Verunreinigungen in der Probe, wie sie beispielsweise durch eine Phasentransformation im Zuge der Ionenimplantation hervorgerufen werden könnten, tragen nicht zum Dämpfungsparameter bei. Zwar haben etwaige Verunreinigungen einen Einfluss auf makroskopische mechanische Eigenschaften, wie zum Beispiel die Streckgrenze oder die elastischen Konstanten, sie beeinflussen allerdings nicht die lokale Umgebung der Sonden mit der jeweils für die untersuchte Phase charakteristischen Quadrupolkopplungskonstante. Alle Informationen, die aus der Verteilung dieses charakteristischen Feldgradienten gezogen werden, stammen somit ausschließlich von Defekten oder Verzerrungen innerhalb der zugehörigen Phase.

Die Annahme, der irreversible Anstieg der Dämpfung sei mit der permanenten Deformation verknüpft, ist nachvollziehbar. So wird für eine signifikante makroskopische Deformation eine Spannung benötigt, die größer als die Streckgrenze des Materials ist. Folglich ist eine PAC-Messung bei einer Spannung $\sigma_{u}$, die größer als die Streckgrenze ist, eine Messung bei der augenblicklichen Fließspannung. Da nun eben diese Spannung und somit die Festigkeit im Experiment steigt, werden im Zuge der Kaltumformung zweifelsohne Versetzungen in das Material eingebracht. Unter Ausnutzung der Proportionalität der Fließspannung zur Wurzel der Versetzungsdichte [67] und der eben gleichen Abhängigkeit der EFGVerteilungsbreite (siehe Kapitel 4.2) wird eine lineare Abhängigkeit der Dämpfung mit der Spannung erwartet [91]. Dies steht im Einklang zum beobachteten irreversiblen Anstieg

${ }^{a}$ Dieses Kapitel basiert im Wesentlichen auf Ergebnissen, die in Referenz [194] veröffentlicht wurden. 
von $\delta$ mit der maximalen Last. Am Beispiel von Abbildung 5.2 wird deutlich, dass auch nach Übersteigen der maximalen Fließspannung im Belastungszyklus Nr. 7 die Dämpfung und somit die Defektdichte noch weiter ansteigen. Die Probe wird so weit verformt, bis die Querschnittsfläche groß genug ist, um der maximalen Fließspannung $\sigma_{u} \approx 1 \mathrm{GPa}$ zu entsprechen.

Es ist darüber hinaus wahrscheinlich, dass auch schon vor Erreichen der makroskopischen Streckgrenze lokal Versetzungsbewegung und -nukleation stattfindet, da die Streckgrenze im Allgemeinen korngrößenabhängig ist und die kritische Schubspannung insbesondere auf der Basalebene der MAX-Phasen sehr klein ist. Ein in diesem Zusammenhang auftretender Anstieg der irreversiblen Verbreiterung konnte hingegen nicht nachgewiesen werden, vermutlich ist die Sensitivität der Dämpfung für solch minimale Änderungen zu gering.

Der Beitrag von Punktdefekten ist aufgrund der unzureichenden Modellierung der Störfunktion konzeptionell nicht von Beiträgen der Versetzungen zu unterscheiden. Es ist also durch die Messung nicht möglich festzustellen, zu welchen Teilen Punkt- und Liniendefekte zum irreversiblen Anstieg der Dämpfung beitragen. Im Falle der MAX-Phasen ist es überraschend, dass schon bei der im Vergleich zur Synthese relativ niedrigen Ausheiltemperatur von $1173 \mathrm{~K}$ die Dämpfung auf das anfängliche Niveau $\delta_{0}$ absinkt, da sich ein Teil der Versetzungen typischerweise in relativ stabilen Versetzungswänden anordnet. Sollten also Versetzungen am irreversiblen Anteil beteiligt sein, handelt es sich hierbei vermutlich um solche, die homogen verteilt oder an Punktdefekten bzw. an anderen Versetzungen gebunden sind und dadurch schon bei niedrigeren Temperaturen den Kristall verlassen bzw. annihilieren können. Gleichzeitig können aber auch durch Versetzungsbewegung erzeugte und bei höheren Temperaturen ausheilende Punktdefekte, beispielsweise auf dem A-Platz der MAX-Phasen, als potentieller Mechanismus der irreversiblen Änderung in Frage kommen. Eine isolierte Betrachtung von homogen verteilten Punktdefekten durch ein Experiment, in dem die MAX-Phase $\mathrm{Ti}_{2} \mathrm{AlC}^{a}$ zunächst an Luft auf $1273 \mathrm{~K}$ erhitzt und anschließend in einem Wasserbad abgeschreckt wird, führt zu keiner merklichen Veränderung der Dämpfung. Die so über das thermodynamische Gleichgewicht hinaus eingefrorenen Punktdefekte sind nicht sichtbar.

Im Folgenden wird unter der Annahme, der irreversible Anstieg der Dämpfung würde allein durch homogen verteilte Versetzungen verursacht, der Anstieg der Versetzungsdichte im Experiment abgeschätzt. Daran anschließend wird die reversible Verbreiterung durch einen quantitativen Vergleich zwischen berechneter elastischer Verbreiterung und experimentellem Anstieg der Dämpfung diskutiert.

\subsubsection{Bestimmung von Versetzungsdichten}

Die Bestimmung der Versetzungsdichte basiert auf den theoretischen Vorarbeiten zu den Gradient-elastischen Konstanten und den Feldgradientenverteilungen aus Kapitel 4. Den

${ }^{a}$ Das gleiche Experiment in $\mathrm{Ti}_{2} \mathrm{AlN}$ führt zu einer Oxidation der Oberfläche bis hin zum implantierten Bereich, womit das PAC-Spektrum seine Aussagekraft verliert. In $\mathrm{Ti}_{2} \mathrm{AlC}$ bleibt aufgrund der außerordentlichen Oxidationsbeständigkeit das PAC-Spektrum auch nach hundertvierzigstündigem Heizen bei $1273 \mathrm{~K}$ unverändert. Ein sich an der Oberfläche bildende Schicht aus $\mathrm{Al}_{2} \mathrm{O}_{3}$ passiviert die darunterliegende MAX-Phase [21]. 


\section{Kapitel 6 Diskussion}

Ausgangspunkt bildet hierbei der quantitative Zusammenhang zwischen Stufenversetzungsdichte und Verteilungsbreite des Feldgradienten aus Gleichung 4.6. Versetzungen mit Schraubencharakter werden vernachlässigt, da sie nur marginal zur gemessenen Dämpfung beitragen. Es wird angenommen, dass in den MAX-Phasen Schraubenversetzungen mit der gleichen Häufigkeit auftreten wie Stufenversetzungen (siehe Kapitel 4.2). Gemischte Versetzungen werden durch die Annahme gleicher Häufigkeiten implizit berücksichtigt. Alle vor der Deformation vorhandenen Defekte, also auch die ursprünglich vorhandenen Versetzungen, bilden die anfängliche Dämpfung $\delta_{0}$. Aufgrund der wurzelförmigen Abhängigkeit der Verteilungsbreite von der Versetzungsdichte folgt

$$
\delta_{\text {irr }}^{2}=\delta^{2}-\delta_{0}^{2}=\left(B_{e}^{z z} \cdot \sqrt{\Delta \rho_{e}}\right)^{2},
$$

wobei $\delta_{\text {irr }}$ der Beitrag zur Dämpfung ist, der durch irreversibel eingebrachte Versetzungen hervorgerufen wird.

Für $\mathrm{Ti}_{2} \mathrm{AlN}$ ändert sich nach einer plastischen Dehnung von ungefähr $3 \%$ die EFGVerteilungsbreite von anfänglich 1,13(5)\% auf 1,33(3)\% (siehe Abbildung 5.2). Dies entspricht unter Ausnutzung der entsprechenden Konstante $B_{e}^{z z}$ einem Anstieg der Versetzungsdichte von $1,3(9) \cdot 10^{15} \mathrm{~m}^{-2}$. Ein ähnliches Bild ergibt sich für die anderen MAXPhasen. In $\mathrm{Nb}_{2} \mathrm{AlC}$ bzw. $\mathrm{Ti}_{2} \mathrm{AlC}$ ergeben sich für eine plastische Dehnung um $2 \%$ ein $\mathrm{Zu}$ wachs von $0,6(4) \cdot 10^{15} \mathrm{~m}^{-2}$ und $0,4(3) \cdot 10^{15} \mathrm{~m}^{-2}$. Im Falle von um $3 \%$ plastisch gedehntem $\mathrm{Ti}_{2}$ InC liegt der Zuwachs bei 1,4(9) $\cdot 10^{15} \mathrm{~m}^{-2}$. Der Fehler ergibt sich zum einen aus der experimentellen Unsicherheit der Dämpfung und zum anderen aus der angenommen Unsicherheit der Gradient-elastischen Konstanten von circa 30\%.

Die Änderung der Versetzungsdichte kann also mit der hier präsentierten Methode ungefähr in einer Größenordnung von $10^{15} \mathrm{~m}^{-2}$ angegeben werden. ${ }^{a}$ Da der Anteil der durch die Deformation eingebrachten Punktdefekte am irreversiblen Anstieg der Dämpfung unberücksichtigt bleibt, handelt es sich bei dieser Angabe um eine obere Schranke der Versetzungsdichte.

Dem stehen Abschätzungen der Versetzungsdichte im Bereich von $10^{14} \mathrm{~m}^{-2}$ gegenüber, die auf TEM-Messungen deformierter MAX-Phasen beruhen [30, 33, 64]. Lokal, insbesondere in Versetzungswänden, ergeben sich zum Teil erheblich höhere Versetzungsdichten. Es ist nicht klar in wie weit die bei den TEM-Messungen angegebenen Versetzungsdichten repräsentativ für das gesamte Material sind, da selektiv ein nur sehr kleiner Bereich der Probe untersucht wird; Versetzungen in ungünstiger Orientierung sind schlimmstenfalls unsichtbar. Zwar ist durch die beschränkte Eindringtiefe der Cadmiumsonde von ungefähr $150 \mathrm{~nm}$ auch bei der PAC-Messung das untersuchte Volumen im Vergleich zum Probenvolumen klein, allerdings führt der makroskopische Implantationsquerschnitt zu einem Messbereich, der um sechs Größenordnungen über dem der TEM-Messung liegt.

\footnotetext{
${ }^{a}$ Bei einer solche Versetzungsdichte würde aus der Taylor-Gleichung $\tau=\alpha G b \sqrt{\rho}(\alpha \approx 0,5)[67]$ und der Annahme eines maximalen Schmid-Faktors, also $\tau=0,5 \cdot \sigma$, mit einem Schubmodul von $120 \mathrm{GPa}$ in $\mathrm{Ti}_{2} \mathrm{AlN}_{\mathrm{N}}$ [200] eine Fließspannung von ungefähr 1 GPa folgen, was ungefähr den erreichten Werten entspricht. Wenngleich die Anwendung dieses Zusammenhangs aufgrund der limitierten Anzahl aktiver Gleitsysteme und der dadurch resultierenden Relevanz anderer Verformungsmechanismen als Versetzungsbewegung fragwürdig ist, so ergibt sich doch ein Hinweis darauf, dass die Größenordnung der hier gemessenen Versetzungsdichten realistisch ist.
} 
Der Vergleich zu einer weiteren großflächigeren Methode wie der hochauflösenden Röntgenbeugung wäre wünschenswert, allerdings wurde in der bisher einzigen dafür in Frage kommenden Studie lediglich die inhomogenen Dehnungen in Form einer Linienverbreiterung angegeben, ohne daraus eine Versetzungsdichte zu extrahieren [19].

\subsubsection{EFG-Verbreiterung unter Last}

Der Vergleich zwischen experimentellem reversiblen Anstieg der Dämpfung und theoretischer Vorhersage der elastische Verbreiterung in Tabelle 5.2 zeigt eine systematische Unterschätzung der erwarteten Verbreiterung. Diese Abweichung wird nachfolgend in Hinblick auf die zugrundeliegenden Annahmen und beteiligten Unsicherheiten diskutiert.

Wird zunächst die Änderung der mittleren Quadrupolkopplungskonstante, $\mathrm{d} \ln v_{Q} / \mathrm{d} \sigma_{u}$, betrachtet, zeigen sich Abweichungen zwischen Theorie und Experiment, die auf einen Fehler der beteiligten Gradient-elastischen Konstanten von ungefähr $40 \%$ hindeuten. Dieser Fehler liegt nur geringfügig über der aus mitunter weniger komplexen Systemen erwarteten Genauigkeit der Gradient-elastischen Konstanten von 30\% (siehe Kapitel 6.2). Erschwerend kommt hierbei hinzu, dass leichte Abweichungen von der regellosen Orientierung einen starken Einfluss auf die Messgröße haben, was insbesondere in Zink zu größeren Abweichungen führt, da hier sowohl in den PAC-Spektren als auch in den Röntgendiffraktogrammen ein erhebliche Vorzugsrichtung der untersuchten Körner beobachtet wird. Alle experimentellen Verläufe liegen konsistent zur theoretischen Erwartung innerhalb der einkristallinen Schranken.

Die gleiche Fehleranalyse der Verbreiterung $\mathrm{d} \delta_{\text {rev }} / \mathrm{d} \sigma_{u}$ zeigt, dass die berechneten Werte erst mit dem Experiment konsistent sind, wenn für die Fehler der Gradient-elastischen Konstanten Werte angenommen werden, die zum Teil erheblich größer sind als alle zuvor ermittelten. So werden in Zink Fehler von $70 \%$, in $\mathrm{Ti}_{2} \mathrm{AlC}$ von $25 \%$, in $\mathrm{Nb}_{2} \mathrm{AlC}$ von $100 \%$, in $\mathrm{Ti}_{2} \mathrm{AlN}$ von $130 \%$ und in $\mathrm{Ti}_{2} \mathrm{InC}$ von $200 \%$ benötigt. Diese erhöhte Abweichung der erforderlichen Fehler in Kombination mit der systematischen Unterschätzung von $\mathrm{d} \delta_{\text {rev }} / \mathrm{d} \sigma_{u}$ impliziert, dass die hier angenommene elastische Verbreiterung nicht den einzigen Beitrag zum experimentell beobachteten reversiblen Anstieg der Dämpfung darstellt.

Zwei grundlegende Mechanismen könnten diesen fehlenden Beitrag erklären. Zum einen ist die Annahme einer uniformen Spannung (Reuss'scher Ansatz [192]) aufgrund der elastischen Anisotropie der untersuchten hexagonalen Systeme nur bedingt anwendbar. ${ }^{a}$ Zum anderen sind, wie oben schon angedeutet, Versetzungen denkbar, die lediglich unter

\footnotetext{
${ }^{a}$ Das andere Extrem wäre der Voigt'sche Ansatz [201], bei dem statt der uniformen Spannung eine uniforme Dehnung angenommen wird. In den Gleichungen 4.10 und 4.11 müssten dafür die Konstanten $C_{m n}$ durch $S_{m n}$ und die Spannung $\sigma_{u}$ durch die Dehnung $\varepsilon_{u}=\sigma_{u} / E$ ersetzt werden, mit dem in der Voigt'schen Approximation berechneten Elastizitätsmodul $E$ des quasi-isotropen Polykristalls. Auch dieser Ansatz liefert nur idealisierte Ergebnisse, allerdings hängt hier die Position des divergierenden Bereich der Wahrscheinlichkeitsdichte (siehe Abbildung 4.11) einzig von der relativ ungenau bestimmten Gradient-elastischen Konstante $S_{31}=-S_{11}-S_{12}$ ab, die insbesondere im Falle der MAX-Phasen sehr kleine Werte annimmt. Beim Reuss'schen Ansatz tritt dieses Problem nicht so stark auf, da hier die Konstante $S_{13}$ das Ergebnis stabilisiert. Aus dem gleichen Grund wird auch auf die häufig verwendete Voigt-Reuss-Approximation verzichtet, bei der ein Mittelwert beider Ergebnisse gebildet werden würde. Eine möglicherweise realitätsnähere Beschreibung könnte mit dem EPSC-Modell gelingen, bei dem individuell orientierte Körner in einem homogenen äußeren Medium unter Berücksichtigung der anisotropen elastischen Eigenschaften deformiert werden [202].
} 


\section{Kapitel 6 Diskussion}

Last existieren bzw. unter Last ihre effektive Länge vergrößern. Beide Ansätze würden die Verteilungsbreite beeinflussen, der zweite aber den Mittelwert der Quadrupolkopplungskonstante unverändert lassen. Eine Kombination beider Mechanismen ist denkbar, deren Unterscheidung aber aufgrund der unzureichenden Modellierung der Störfunktion nicht möglich ist.

Reversibel unter Last entstehende Beiträge von Versetzungen sind beispielsweise in Form einer Ausbeulung schon bestehender Versetzungslinien an Defekten oder durch eine unter Last einsetzende Versetzungsnukleation bzw. -multiplikation in Kombination mit einer Versetzungsannihilation nach Wegnahme der Last durchaus mit klassischer Versetzungstheorie vereinbar. Ein vergleichbarer reversibler Anstieg der Versetzungsdichte wurde beispielsweise an über die Streckgrenze deformierten nanokristallinem Nickel durch in-situ Röntendiffraktometriemessungen nachgewiesen [203]. Der Anteil der unter Last zusätzlich auftretenden Versetzungen an der reversiblen Reflexverbreiterung wurde, konsistent mit den hier gezeigten Ergebnissen, mit ungefähr 50\% abgeschätzt [203]. Da es sich bei dem speziellen Beispiel um ein kubisches System handelt, spielen elastische Anisotropien und somit Abweichungen des uniformen Spannungszustandes nur eine untergeordnete Rolle. Es ist anzunehmen, dass aus diesem Grunde eine Erwähnung dieses Aspektes in Referenz [203] ausblieb.

Im Hinblick auf den kontrovers diskutierten IKB-Mechanismus, der reversible Hysteresen im Spannungs-Dehnungs-Diagramm erklären soll, kann somit keine Aussage getroffen werden. Zwar stünde dieser Mechanismus mit der zweiten Erklärung im Einklang, da hierzu Versetzungen postuliert werden, die unter Last in Form von Versetzungsringen entstehen und nach der Wegnahme der Last vollständig annihilieren [18], allerdings lassen sich diese Beiträge im Sinne der Dämpfung nicht von anderen reversiblen Beiträgen unterscheiden. Umgekehrt lässt sich aber auch der dazu konkurrierende Ansatz von Jones et. al. [19] nicht verifizieren, der die Hysteresen nicht auf den IKB-Mechanismus, sondern auf eine Kombination aus elastischer Verformung in Körnern niedrigen Schmid-Faktors und klassischer Versetzungsbewegung in Körnern hohen Schmid-Faktors zurückführt. Dieser Ansatz würde zu keinem zusätzlichen Beitrag der reversiblen Verbreiterung unter Last führen; gleichwohl ist parallel eine reversible Erhöhung der Versetzungsdichte wie im Beispiel von Budrovic [203] möglich. Die für beide Mechanismen notwendige Mobilität der Versetzungen bei niedrigen Spannungen ist hingegen wohlbekannt [31,37], sodass auch schon vor der makroskopischen Streckgrenze des Materials Versetzungsbewegung auf der Basalebene stattfinden kann. Eine potentielle Abweichung vom uniformen Spannungszustand spielt in keiner der beiden Theorien eine Rolle.

Der fehlende Beitrag kann analog zur Abschätzung im vorherigen Abschnitt mit einer zusätzlichen Versetzungsdichte unter Last in Verbindung gebracht werden. Der dabei nicht berücksichtigte Einfluss inhomogener Spannungen ist für die MAX-Phasen vermutlich eher klein, da die elastische Anisotropie selbst relativ klein ist (vgl. $c_{11}$ und $c_{33}$ in Tabelle 2.1). Für eine exemplarische Last von 0,5 GPa ergäbe sich so ein reversibler Anstieg der Versetzungsdichte, der zwischen $1,3(9) \cdot 10^{14} \mathrm{~m}^{-2}$ für Ti 2 AlC und $1,0(8) \cdot 10^{15} \mathrm{~m}^{-2}$ für Ti 2 InC liegen würde. Die Fehler ergeben sich aus den experimentellen Unsicherheiten der reversiblen Änderung der Dämpfung, der Unsicherheit der Konstante $B_{e}^{z z}$ und der Unsicherheit der theoretischen Vorhersage der elastischen Verbreiterung. Unsicherheiten aufgrund 
des unbekannten Anteils der Punktdefekte entfallen hingegen. Zum Vergleich: Die Versetzungsdichte steigt in nanokristallinem Nickel unter einer Last von $2 \mathrm{GPa}$ reversibel von $4,7 \cdot 10^{15} \mathrm{~m}^{-2}$ auf $6,5 \cdot 10^{15} \mathrm{~m}^{-2}$ an [203]; es ist zu erwarten, dass der Effekt in grobkörnigeren Materialien erheblich geringer ausfallen würde, was im Einklang zu den hier beobachteten Effekten stünde.

\subsubsection{Dünnschichtproben}

Die $\mathrm{Cr}_{2}$ AlC-Dünnschichtproben zeigen im Vergleich zur Volumenprobe keinen Unterschied in der Quadrupolkopplungskonstante oder der Dämpfung der Störfunktion. Es gibt keinen Hinweis auf eine zusätzliche interne Spannung in der Schicht, weder aufgrund von Defekten noch aufgrund von Grenzflächeneffekten; selbst Temperaturänderungen führen zu keiner messbaren thermisch induzierten Spannung. Die Vermutung liegt nahe, dass etwaige Spannungen, die an der Grenzfläche zum Siliziumnitrid entstehen, über die Korngrenzen hinweg vollständig abgebaut werden und somit von den PAC-Sonden, die sich einen Mikrometer über der Grenzfläche befinden, nicht mehr detektiert werden. Obwohl $\mathrm{Cr}_{2} \mathrm{AlC}$ theoretisch nicht behandelt wurde und somit keine Gradient-elastischen Konstanten für diese MAX-Phasen vorliegen, kann angenommen werden, dass das Fehlen interner Spannungen in den $\mathrm{Cr}_{2} \mathrm{AlC}$-Filmen auf alle polykristallinen Dünnschichtsysteme der MAX-Phasen vergleichbarer Schichtdicke zutreffen würde. Es ist also generell möglich, Dünnschichtsysteme der MAX-Phasen so herzustellen, dass sie - wie Volumenproben einen spannungsfreien Zustand erreichen.

Die Tatsache, dass sich der gemessene EFG in Dünnschichtsystemen der MAX-Phasen im Vergleich zu Volumenproben nicht verändert und ein signifikanter Anteil der PACSonden auf dem A-Platz eingebaut wird, erlaubt es Dünnschichtsysteme in gleicher Weise mit der PAC-Methode zu untersuchen wie Volumenproben. Eine besonders interessante Anwendung ergibt sich hierbei in Hinblick auf die potentiell magnetischen MAX-Phasen $\left(\mathrm{Cr}_{1-x}, \mathrm{Mn}_{x}\right)_{2} \mathrm{AlC},\left(\mathrm{Cr}_{1-x}, \mathrm{Mn}_{x}\right)_{2} \mathrm{GaC}$ und $\left(\mathrm{Cr}_{1-x}, \mathrm{Mn}_{x}\right)_{2} \mathrm{GeC}$ [11, 204-210], von denen aussichtsreiche Kandidaten in Dünnschichtsystemen synthetisiert wurden. Die magnetische Ordnung sollte in solchen Phasen mit der PAC-Methode über die magnetische Hyperfeinwechselwirkung in Form einer mit der elektrischen Hyperfeinwechselwirkung kombinierten Störung sichtbar sein.

\subsection{Störungen fernab der linear-elastischen Theorie}

Die bisher diskutierten Effekte beschränkten sich auf langreichweitige Dehnungsfelder, die innerhalb des kleinen, für den Feldgradienten aber maßgeblichen Bereichs, als konstant angenommen werden können. Effekte die sich innerhalb dieses kleinen Bereichs abspielen, können a priori nicht mehr kontinuumstheoretisch beschrieben werden; die linearelastische Theorie versagt.

Die Untersuchung der Mischkristallsysteme fällt genau in den Zwischenbereich, da in Mischkristallen einerseits mit hoher Wahrscheinlichkeit die unmittelbare Umgebung des Kerns, der den Feldgradienten misst, im Vergleich zur reinen Phase unterschiedlich besetzt ist, andererseits aber auch die Gitterkonstanten - gemäß der Vegard'schen Regel - 


\section{Kapitel 6 Diskussion}

einer Veränderung unterliegen, die in Form eines Dehnungstensors beschrieben werden kann. Es ist davon auszugehen, dass sich beide Beiträge überlagern, jedoch kann allein der Einfluss einer unterschiedlichen Umgebung individueller Sondenkerne die beobachtete Verbreiterung der Feldgradientenverteilung hervorrufen. Änderungen der Gitterkonstante würden lediglich zu einer uniformen Änderung der Feldgradientenstärke führen (siehe Kapitel 2.2.4). Da die Konzentration $x$ in den hier untersuchten Mischkristallen bei 0,5 liegt, beschränkt sich die Diskussion im Wesentlichen auf die lokale Umgebung der Sondenkerne. Von Bereichen, in denen lineare Änderungen der Messgrößen ausgehend von den reinen Phasen erwartet werden, sind diese Experimente maximal entfernt.

Die hier beobachteten Störfunktionen, die mit einer einzigen Quadrupolfrequenz hoher Verteilungsbreite beschrieben werden können, implizieren in Übereinstimmung mit den Röntgenstrukturmessungen, dass es sich bei den untersuchten Systemen um homogene Mischkristalle handelt. Die Bildung eines geordneten MAX-Phasen-Mischkristalls kann bei Temperaturen oberhalb von $12 \mathrm{~K}$ ausgeschlossen werden. Eine entsprechende Ordnung würde in einer deutlich abgeschwächten Dämpfung des PAC-Spektrums resultieren, die in keiner der untersuchten Systeme beobachtet werden konnte. Die ermittelten Quadrupolkopplungskonstanten und somit mittleren Feldgradientenstärken liegen innerhalb der Stärken der zugehörigen reinen Phasen, folgen aber keiner linearen Interpolation, wie es bei den Gitterkonstanten der Fall ist (siehe Kapitel 5.3).

Vergleichbare Abweichungen des EFGs von der linearen Interpolation wurden bereits in Hafnium-Zirkonium-Mischkristallen [105] beobachtet, allerdings überstieg hier die ermittelte mittlere Feldgradientenstärke sogar die Stärke der reinen Phasen ${ }^{a}$. Im Gegensatz dazu stehen Experimente in Metalloxiden, die eine vollständig lineare Interpolation zulassen [103, 104]. Da systematische Fehler im Falle der MAX-Phasen ausgeschlossen werden können (siehe Kapitel 5.3), stellt sich die Frage, warum sich der mittlere Feldgradient in manchen Mischkristallsystemen linear interpolieren lässt, in anderen jedoch nicht. Hierfür ist es notwendig, den Ursprung der beobachteten Effekte zu verstehen.

Der in den Metalloxiden beobachtete lineare Verlauf sei nach Peterson [103] unmittelbar in der linearen Abhängigkeit der Gitterkonstanten und der internen Positionen mit dem Anteil $x$ begründet. Diese Schlussfolgerung beruht auf Rechnungen im Punktladungsmodell, die neben der Größenordnung des Feldgradienten auch den zum Experiment vergleichbaren linearen Verlauf des Feldgradienten vorhersagen. Wie allerdings bereits oben angedeutet, führt eine solche Betrachtung zu keiner Verteilung des Feldgradienten. Die dennoch beobachtete Verteilung führte Peterson [103], ohne näher auf den tatsächlichen Ursprung einzugehen, auf die Unordnung der nächsten Nachbarn zurück. Implizit wurde dabei angenommen, dass sich der lokale EFG abhängig von der Anzahl der Fremdatome in nächster Nachbarschaft linear bis hin zum EFG der anderen reinen Phase ändert. Die Wahrscheinlichkeit, eine bestimmte Anzahl Fremdatome zu finden, wäre hierbei durch die Binomialverteilung gegeben; folglich würde die Binomialverteilung einer durch den Anteil $x$ festgelegten Wahrscheinlichkeit die Verteilung des Feldgradienten widerspiegeln.

\footnotetext{
${ }^{a}$ Die im Hafnium-Zirkonium-Mischkristall [105] beobachtete Feldgradientenverteilung ist so breit $(\delta=$ $20 \%$ ), dass die mittlere Feldgradientenstärke vermutlich erheblich überschätzt wurde [164]. Die Abweichung von der linearen Interpolation ist also vermutlich in diesem System nicht ganz so stark, wie die dort angegebenen Feldgradientenstärken suggerieren.
} 
Diese Interpretation ist im Hinblick auf die hier durchgeführten Experimente nicht haltbar. Zum einen widerspricht ihr die beobachtet Abweichung vom linearen Verhalten, zum anderen die in den Mischkristallen beobachtete Verteilungsbreite.

Der erste Einwand könnte prinzipiell dadurch entkräftet werden, dass in den hier durchgeführten PAC-Messungen mit der Indium-Cadmiumsone ein Element zum Einsatz kommt, das in den meisten untersuchten Mischkristallen nicht nativ enthalten ist. Es wäre denkbar, dass sich um das fremde Indium bevorzugt Atome einer Sorte des Mischkristalls sammeln und sich dadurch obige Binomialverteilung in Richtung einer reinen Phasen verschiebt. Die an ohnehin im Mischkristall enthaltenen Sonden durchgeführten NMR-Messungen der Metalloxide würden diese Vorzugsrichtung selbstverständlich nicht zeigen, und auch die einzige MAX-Phase, die annähernd eine lineare Interpolation zulässt, enthält mit Indium nativ die verwendete PAC-Sonde. Gleiches müsste dann konsequenterweise aber auch für die Hafnium-Zirkonium-Mischkristalle gelten, da in diesem Falle das Hafnium die PAC-Sonde darstellt - das Gegenteil wird beobachtet.

Der zweite Einwand lässt sich hingegen nicht relativieren. Würde sich die Feldgradientenstärke tatsächlich linear mit der Anzahl fremder Atome in nächster Nachbarschaft verändern, dürften die beobachteten Frequenzverteilungen keinesfalls über die durch die reinen Phasen vorgegebenen Ränder hinausragen. Am Beispiel $\mathrm{Ti}_{2}\left(\mathrm{Al}_{0,5}, \mathrm{In}_{0,5}\right) \mathrm{C}$ wird deutlich, dass das Gegenteil der Fall ist (siehe Abbildung 5.11): Die Standardabweichung der Frequenzverteilung übersteigt mit 4,7(2)\%, also 13,7(6) MHz, deutlich den Abstand zwischen der Quadrupolkopplungskonstante des reinen Systems und der des gemischten Systems von 7,9(7) MHz (siehe Tabelle 5.4).

Erst die Annahme einer rein von der Anzahl der fremden nächsten Nachbarn unabhängigen lokalen Feldgradientenstärke erlaubt es, alle experimentellen Daten konsistent zu erklären. Der Schlüssel besteht - wie auch bei den Gradient-elastischen Konstanten - in den Auswirkungen der lokalen Umgebung auf die asymmetrische Besetzung der $p$ - und $d$ Unterzustände. Die Auswirkungen auf den lokalen Feldgradienten sind systemspezifisch und im Allgemeinen nicht auf das Intervall zwischen den reinen Phasen beschränkt. Bestimmte Konfigurationen sind also in der Lage, eine große Spanne von Feldgradienten abzudecken. Die Sensitivität auf kleine Änderungen der lokalen Umgebung ist groß, da es sich bei der für den EFG relevanten asymmetrischen Besetzung um eine Bilanz vergleichbarer Größen handelt.

Die Verteilung der Feldgradienten aller Konfigurationen muss bei $x=0,5$ keineswegs symmetrisch sein, Abweichungen von der linearen Interpolation sind also vermutlich die Regel. Eine lineare Interpolation wird in diesem Zusammenhang genau dann erwartet, wenn die Feldgradientenstärke einer bestimmten Konfiguration den gleichen Abstand zur reinen Phase aufweist wie die invertierte Konfiguration von der anderen. Dies scheint im Falle der Metalloxide der Fall zu sein. Entsprechende DFT-Rechnungen unterschiedlicher Konfigurationen wären in diesem Kontext hilfreich, allerdings im Falle der MAX-Phasen außerordentlich komplex.

Diese Interpretation steht im Einklang zu den Unterschieden der beobachteten Feldgradientenverteilungen innerhalb der verschiedenen MAX-Phasen-Mischkristalle. So steigt zwar zweifelsohne die Dämpfung der Störfunktionen mit zunehmenden Abstand der Feldgradientenstärken der reinen Phasen an (vgl. $\left(\mathrm{Ti}_{1-x}, \mathrm{Nb}_{x}\right)_{2} \mathrm{AlC}$ und $\left(\mathrm{Ti}_{1-x}, \mathrm{~V}_{x}\right)_{2} \mathrm{AlC}$ in Abbil- 


\section{Kapitel 6 Diskussion}

dungen 5.14a und 5.15), allerdings spielt auch die ersetzte Atomsorte eine erhebliche Rolle.

Wird das System $\mathrm{Ti}_{2}\left(\mathrm{Al}_{1-x}, \mathrm{In}_{x}\right) \mathrm{C}$ betrachtet, ergibt sich eine extreme Verbreiterung, obwohl der entsprechende Abstand nur gering ist. Dies ist eine direkte Konsequenz des Ursprunges des EFGs, der in den MAX-Phasen durch die asymmetrische Besetzung der $p_{x^{-}}$, $p_{y}$ - und $p_{z}$-Orbitale bestimmt wird, wovon die ersten beiden unmittelbar von der Bindung zu den umliegenden und hier durchmischten A-Elementen beeinflusst werden [46]. Dem entgegen zeigt sich in $\mathrm{Ti}_{2} \mathrm{Al}\left(\mathrm{C}_{1-x}, \mathrm{~N}_{x}\right)$ bei vergleichbarem Abstand der Feldgradienten der reinen Systeme nur einer geringe Verbreiterung, da derX-Platz der MAX-Phase mit der Sonde keine Bindung eingeht und somit auf dessen p-Unterorbitale keinen direkten Einfluss hat [46]. In den MAX-Phasen-Mischkristallen des M-Platzes besteht ein direkter Einfluss auf das $p_{z}$-Orbital [46], die beobachteten Dämpfungen sind im Vergleich zu den anderen Mischkristallen und dem jeweiligen Abstand der Feldgradienten der reinen Phasen mittelmäßig stark.

In der Theorie von Peterson würden die Dämpfungen auf dem M- und auf dem A-Platz vergleichbar groß sein, da beide Atomsorten jeweils sechs der zwölf nächsten Nachbarn stellen $^{a}$ - das Gegenteil ist der Fall. Der X-Platz hingegen würde auch hier eine Sonderstellung einnehmen, da die sechs nächsten X-Atome abgeschirmt in der zweiten Koordinationssphäre liegen.

Die Temperaturabhängigkeit der Quadrupolkopplungskonstante bzw. der Dämpfung gibt keinen weiteren Hinweis auf den Ursprung der beobachteten Effekte. In beiden Theorien würde sich der Verlauf der Quadrupolkopplungskonstante der Mischkristalle in den Verlauf der reinen Phasen wie beobachtet einbetten (siehe Abbildung 5.13). Auch die mit ansteigender Temperatur rückläufige Verteilungsbreite wird in beiden Theorien erwartet; bei sich schneidenden Verläufen der Quadrupolkopplungskonstante ${ }^{b}$ wird die Dämpfung, wie erwartet, minimal (siehe Kapitel 5.3).

\subsection{Methoden zur Bestimmung interner Spannungen im Vergleich}

Mit den in dieser Arbeit entwickelten Werkzeugen und Methoden lässt sich die gestörte $\gamma$ - $\gamma$-Winkelkorrelation zur quantitativen Untersuchung von internen Spannungen in einer Vielzahl von Materialien einsetzen. Darüber hinaus können durch die lokale Änderung der elektronischen Zustandsdichte kurzreichweitige Einflüsse von Fremdatomen in Mischkristallsystemen studiert werden. Die dabei relevanten Feldgradientenverteilungen lassen sich alternativ zur PAC-Methode auch mit der NMR- oder der Mößbauer-Spektroskopie bestimmen. Wesentliche Unterschiede zwischen den Methoden bilden die unterschiedlichen Anforderungen an das jeweilige Sondenatom und der Raum - Frequenzraum oder Zeitraum - in dem die Quadrupolwechselwirkung gemessen wird. Einen dazu komplementären An-

\footnotetext{
${ }^{a}$ Der Abstand des M-Platzes vom Sondenatom ist, am Beispiel von $\mathrm{Ti}_{2} \mathrm{AlC}$, mit 2,90 $\AA$ sogar kleiner als der Abstand zum A-Platz mit 3,07 A, sodass dessen Einfluss sogar leicht größer sein sollte. Der X-Platz ist hingegen mit $3,87 \AA$ weit von der Sonde entfernt.

${ }^{b}$ Die Gitterkonstanten sind bei dieser speziellen Temperatur von $1200 \mathrm{~K}$ unterschiedlich. Es wird folglich auch nicht erwartet, dass sich andere Materialeigenschaften bei dieser Temperatur gleichen.
} 
satz bieten Diffraktometriemethoden, die durch eine Messung der Reflexverbreiterung auf interne Spannungen schließen lassen.

Unabhängig von der verwendeten Methode werden interne Spannungen, die auf Defekte zurückgehen, üblicherweise als inhomogene Dehnungen bezeichnet, die daraus resultierende Verbreiterung als Defektverbreiterung. Die durch eine äußere Spannung hervorgerufene Verbreiterung einer Messgröße wird als elastische Verbreiterung bezeichnet, die damit einhergehende Verzerrung als elastisch inhomogene Dehnung.

Ein grundlegender Unterschied zwischen Methoden, die auf einer Messung der Quadrupolwechselwirkung beruhen, und jenen, die auf einer Messung der kohärenten Streuung an Netzebenen basieren, liegt in ihrer Sensitivität auf Punktdefekte. So sind bei Diffraktometriemethoden Punktdefekte praktisch unsichtbar, da die zugehörigen Dehnungsfelder zu einer inkohärenten Streuung der einfallenden Strahlung führen. Punktdefekte tragen damit nur zum Untergrund bei [211]. Die Reflexverbreiterung durch den übrigbleibenden sichtbaren Teil der internen Spannungen weist eine Abhängigkeit vom Streuwinkel auf, die es erlaubt, den Anteil der Reflexverbreiterung, der durch die endliche Kristallitgröße in Form der Scherrer-Gleichung entsteht, vom Anteil der internen Spannung abzutrennen; die zugehörige Darstellung erfolgt im Williamson-Hall-Diagramm [176] bzw. durch eine Warren-Averbach-Analyse [177]. Prinzipiell gelingt bereits dadurch eine rudimentäre Zuordnung von Versetzungsdichten, allerdings kann die jeweilige Analyse durch einen modifizierten Ansatz verfeinert werden [212]. Die wesentliche Erweiterung des modifizierten Ansatzes besteht in der Berücksichtigung eines sogenannten Kontrastfaktors, der dem Einfluss unterschiedlicher Versetzungen zur winkelabhängigen Verbreiterung Rechnung trägt. Der Kontrastfaktor hängt maßgeblich von den elastischen Eigenschaften des Materials und dem Charakter der Versetzung ab. Für kubische und hexagonale Systeme gelingt dessen Bestimmung numerisch [213]. Grundlage einer solchen Analyse sind hochauflösende Diffraktogramme, wie sie beispielsweise an eine Synchrotronstrahlungsquelle oder anderen speziell dafür ausgelegten Diffraktometern gemessen werden können.

Die Rolle des Kontrastfaktors übernimmt bei Methoden, die auf der Quadrupolwechselwirkung basieren, eine Analyse der Gradient-elastischen Konstanten und der defektspezifischen Feldgradientenverteilung wie in Kapitel 4. Anstelle der starken Röntgenquelle tritt die jeweilige Sonde, die selektiv auf definierten Gitterplätzen eingebaut werden kann und so beispielsweise Messungen des Spannungsfeldes in Quantenpunkten ermöglicht [94-96]. Die Möglichkeit, verschiedene Anteile der Verbreiterung separat zu betrachten ist hingegen nicht gegeben, vielmehr kumulieren sich alle Beiträge in einem einzigen Parameter, der Feldgradientverbreiterung. Dieser Umstand birgt, wie oben an den PAC-Messungen gesehen, eine gewisse Mehrdeutigkeit der Ergebnisse. Da aber die Form der einzelnen Verteilungen prinzipiell bekannt ist, ließen sich durch eine geeigneten Modellierung der Störfunktion und bei hinreichend guter Statistik im Idealfall die einzelnen Beiträge rekonstruieren und somit vergleichbare Informationen zum modifizierten Williamson-HallDiagramm extrahieren - mit zusätzlichen Informationen über in der Probe enthaltene Punktdefekte.

Die Sensitivität des Feldgradienten auf Dehnungsfelder ist, wie an der Volumen- und Strukturabhängigkeit gezeigt, zum Teil überproportional groß, womit sich auch schon kleinste Dehnungen in der Quadrupolwechselwirkung bemerkbar machen können. Der 
Einfluss von nächsten Nachbarn auf die elektronische Struktur lässt sich hingegen nicht durch eine elastische Theorie beschreiben und ist dadurch gänzlich unzugänglich für Diffraktometriemethoden. In dieser Hinsicht erscheint für viele Fragestellungen eine kombinatorische Analyse aus Methoden, welche die Quadrupolwechselwirkung nutzen, und Diffraktometriemessungen sinnvoll, da sich die verschiedenen Methoden in Teilgebieten komplementär zueinander verhalten. 


\section{Kapitel 7}

\section{Zusammenfassung und Ausblick}

Basierend auf dem Konzept des Gradient-elastischen Tensors [48] wurde ein Weg vorgestellt, mit Methoden der nuklearen Festkörperphysik interne Spannungen in beliebig komplexen Kristallen zu bestimmen. Bisher waren solche Untersuchung auf kubische Systeme limitiert, die zusätzlich als Einkristall vorliegen müssen [91]. Diese Limitierung konnte durch Berechnungen der Gradient-elastischen Konstanten mit Hilfe der Dichtefunktionaltheorie überwunden werden. Die Anwendung dieses Konzeptes auf die MAX-Phasen ermöglichte es, in-situ interne Spannungen im Material zu detektieren und dabei auftretende Versetzungsdichten abzuschätzen.

Die durch diesen Ansatz berechneten Gradient-elastischen Konstanten weisen eine Genauigkeit von ungefähr $30 \%$ auf. Die theoretische Behandlung dieses Problems ermöglichte es erstmals, die Ursache der Dehnungsabhängigkeit des elektrischen Feldgradienten auf die dehnungsabhängige Änderungen der asymmetrischen Besetzung der p- und d-Unterzustände der jeweiligen Sonde zurückzuführen. Es zeigte sich, dass Vorhersagen der Gradient-elastischen Konstanten im Allgemeinen nicht möglich sind, da sich die Änderung aus zum Teil gegenläufigen Beiträgen der einzelnen Unterzustände zusammensetzt. Einzig die hier gezeigte, vollständige Berechnung der elektronischen Struktur erlaubt es, die Konstanten mit genannter Genauigkeit zu ermitteln.

Unabhängig von der Untersuchung interner Spannungen ermöglichte es dieser Ansatz, unmittelbar Volumen- und Strukturabhängigkeiten zu bestimmen. Es konnte zum ersten Mal auf direktem Wege gezeigt werden, dass die Vorstellung eines in der Nähe ideal dichtester Packung verschwindenden elektrischen Feldgradienten [89] zutrifft. Änderungen des Volumens führen, wie zuvor vermutet, zu einer überproportionalen Änderung des Feldgradienten; die Stärke dieser Änderung ist jedoch kleiner, als aufgrund indirekter Messungen vermutet wurde [89]. Eine isotrope Volumenänderung führt in nicht-kubischen Systemen im Allgemeinen also zu einer anisotropen Änderung der Elektronendichte.

Ferner gelang es erstmals in hexagonalen Systemen, Vorhersagen für die Verteilung des Feldgradienten zu entwickeln, wie sie in der Umgebung bestimmter Defekte oder unter dem Einfluss einer elastischen Verbreiterung zu erwarten sind. Eine explizite Trennung der einzelnen Beiträge gelang im Experiment jedoch nicht, da hierfür weder die Statistik genügte noch eine exakte Modellierung der gemessenen Störfunktion im Hinblick auf die Verteilung des Asymmetrieparameters zur Verfügung stand. Trotz dieser Einschränkung konnte eine obere Abschätzung der bei einer Verformung in das Material eingebrachten Versetzungsdichte angegeben werden. Die eingebrachten Versetzungen entsprechen im Falle der MAX-Phasen einem Anstieg der Versetzungsdichte von ungefähr $10^{14} \mathrm{bis} 10^{15} \mathrm{~m}^{-2}$. Des 
Weiteren zeigte eine Analyse der reversibel unter Last auftretenden Verbreiterung des Feldgradienten, dass neben der durch homogene Spannungen hervorgerufenen elastischen Verbreiterung auch andere Beiträge existieren. Diese setzen sich zum einen aus einer reversibel unter Last erhöhten Versetzungsdichte und zum anderen aus dem Einfluss inhomogener Spannungen, die auf die elastische Anisotropie zurückzuführen sind, zusammen.

Das Problem limitierter Statistik und ungenügender Beschreibung der Störfunktion könnte durch den kombinierten Einsatz eines Spektrometers nächster Generation [148] und neu entwickelter Auswerteroutinen [169] überwunden werden. Vielversprechend wäre es hierbei, die Störfunktion ausgehend von einer vorgegebenen Feldgradientenverteilung, die sowohl die Stärke als auch den Asymmetrieparameter umfasst, zu simulieren. Die dafür nötigen Grundlagen stehen mit den hier bestimmten Gradient-elastischen Konstanten und den daraus abgeleiteten Verteilung erstmals bereit. Sollte es in einer solchen weiterentwickelten Analyse der Messdaten möglich sein, Beiträge von Punktdefekten und Versetzungen zu separieren, gelängen präzisere Aussagen über die beteiligten Defektdichten.

Darüber hinaus lassen sich die hier hergeleiteten Feldgradientenverteilungen auch direkt in anderen Methoden, wie beispielsweise der NMR- oder Mößbauerspektroskopie, zur quantitativen Beschreibung von internen Spannungen verwenden. Ein interessantes Beispiel bildet hierbei die bereits erwähnte Untersuchung von InP/GaInP-, InGaAs/GaAsQuantenpunkten mittels der magnetischen Kernresonanzspektroskopie [94-96], bei der es teilweise sogar ortsaufgelöst gelingt, das Spannungsfeld des Quantenpunkts zu vermessen. Auch hier kann mit den in dieser Arbeit entwickelten Methoden die Limitierung auf kubische Systeme überwunden werden. Potentielle Systeme umfassen hierbei CdSe/ZnSe-, CdSe/ZnS-, CdS/HgS- und ZnO-Quantenpunkte [214-217] sowie GaN/AlN- und InN/AlNQuantenpunkte [218, 219].

In den aufgeführten II-VI- bzw. III-V-Halbleitern könnte mit diesem Ansatz darüber hinaus auch jede andere Form interner Spannungen vermessen werden. Die entsprechende Feldgradientenverteilung würde dann zuvor analog zu den hier durchgeführten Simulationen ermittelt werden müssen. Gleichermaßen interessant wären entsprechende Studien in technologisch relevanten hexagonalen Metallen, wie beispielsweise Magnesium, Titan oder Zirkon. In Frage kommende PAC-Sonden wären beispielsweise durch ${ }^{28} \mathrm{Mg} /{ }^{28} \mathrm{Al}$, ${ }^{44} \mathrm{Ti} /{ }^{44} \mathrm{Sc},{ }^{72} \mathrm{Zn} /{ }^{72} \mathrm{Ga},{ }^{111 \mathrm{~m}} \mathrm{Cd} /{ }^{111} \mathrm{Cd}$ oder eben durch die hier verwendete ${ }^{111} \mathrm{In} /{ }^{11} \mathrm{Cd}$-Sonde gegeben [149]. Dabei ist es insbesondere bei der Untersuchung von Nanostrukturen hilfreich, dass die radioaktiven Sonden gezielt in das Materialsystem, beispielsweise in Form einer Monolage [172], eingebracht werden können. Durch Verwendung der NMR- oder Mößbauerspektroskopie werden die Möglichkeiten noch weiter vergrößert, da hier konzeptionell bedingt andere Sonden und Geometrien zum Einsatz kommen können.

Die an den Mischkristallen durchgeführten Experimente verdeutlichen - auch ohne explizite Berechnung der elektronischen Struktur - abermals die Relevanz der asymmetrischen Besetzung der p- und d-Unterzustände für den EFG. Es zeigte sich, insbesondere bei der in dieser Arbeit neu entdeckten MAX-Phase $\mathrm{Ti}_{2}\left(\mathrm{Al}_{0,5}, \mathrm{In}_{0,5}\right) \mathrm{C}$, deutlich, dass die dabei relevante Asymmetrie in hohem Maße sensitiv auf eine lokale Störung in der Basalebene reagiert. In diesem Zusammenhang wäre es wünschenswert, zumindest einige ausgewählte Konfigurationen nächster Nachbarn ab-initio zu berechnen, um die beobachtete 
Verbreiterung detailliert verstehen zu können. Die hier durchgeführten Messungen zeigen deutlich, dass es sich bei den MAX-Phasen-Mischkristallen um vollständig homogene Substitutionsmischkristalle handelt; eine lokale Ordnung oder gar eine Phasenseparation kann ausgeschlossen werden.

Ein weiteres Forschungsfeld eröffnet sich durch die in dieser Arbeit gezeigte Möglichkeit, Dünnschichtsysteme mit der PAC-Methode zu charakterisieren. In polykristallinen Schichten von $1 \mu \mathrm{m}$ Dicke ist demnach keine Veränderung des PAC-Signals zu erwarten, womit der Fokus auf andere Effekte, wie etwa Magnetismus, gelegt werden kann. Die dafür in Frage kommenden Systeme $\left(\mathrm{Cr}_{1-x}, \mathrm{Mn}_{x}\right)_{2} \mathrm{AlC},\left(\mathrm{Cr}_{1-x}, \mathrm{Mn}_{x}\right)_{2} \mathrm{GaC}$ und $\left(\mathrm{Cr}_{1-x}, \mathrm{Mn}_{x}\right)_{2} \mathrm{GeC}$ $[11,204-210]$ müssten bei einer Untersuchung mit der ${ }^{111} \mathrm{In} /{ }^{111} \mathrm{Cd}$-Sonde hierzu in entsprechender Schichtdicke synthetisiert werden, da dünnere Schichten möglicherweise zu einer ungewollten Dämpfung der Störfunktion führen. In jedem Falle wäre die magnetische Hyperfeinwechselwirkung durch eine Kombination mit der elektrischen Quadrupolwechselwirkung sichtbar. Einerseits hat dies den Vorteil, dass die charakteristische Wechselwirkungsfrequenz des A-Platze genutzt werden kann, um festzustellen, ob die Sonde auf dem gewünschten Platz eingebaut ist, andererseits erschwert die kombinierte Wechselwirkung die Interpretation der Störfunktion erheblich ${ }^{a}$.

Ein möglicher Kandidat wäre hierbei die Sonde ${ }^{48} \mathrm{Cr} /{ }^{48} \mathrm{~V}$ [149], die nativ den M-Platz der obigen MAX-Phasen besetzten sollte. Der Einbau der Sonde könnte durch Ionenimplantation am GLM-Zweig von ISOLDE (CERN) [148] erfolgen. Die Implantationsenergie sollte dabei so gewählt werden, dass eine ähnliche Eindringtiefe erreicht wird, wie bei den in dieser Arbeit verwendeten ${ }^{111} \mathrm{In} /{ }^{111} \mathrm{Cd}$-Sonden. Auf diese Weise kann ausgeschlossen werden, dass sich die Sonden nach dem Ausheilen der Implantationsschäden möglicherweise in einer sich bildenden Oxidschicht befinden [47].

Die Auswirkungen der magnetischen Ordnung auf das Hyperfeinfeld am Kernort der verwendeten Sonde könnten zuvor mit Hilfe der Dichtefunktionaltheorie theoretisch untersucht werden [123]. Hierbei sind für verschiedenen Sonden stark unterschiedliche Felder zu erwarten, da das Hyperfeinfeld am Kernort durch eine Wechselwirkung der Elektronenhülle mit der Umgebung verstärkt oder geschwächt wird. In Elementen der seltenen Erden ist dieser Effekt teilweise besonders stark [123, 220], sodass es sich unter Umständen anbietet, solche PAC-Sonden zur Untersuchung zu verwenden.

${ }^{a}$ Einen möglichen Ausweg, die kombinierte Wechselwirkung zu vermeiden, bietet die Verwendung einer Sonde, die kein Quadrupolmoment besitzt und damit auch keine Quadrupolwechselwirkung zeigt. 

Anhang 



\section{Anhang A}

\section{Details der DFT-Rechnungen}

Die Dichtefunktionalrechnungen in dieser Arbeit wurden mit WIEN2k [118], Version 13.1, durchgeführt. Als Austausch-Korrelations-Potential kam für alle Systeme die Gradientennäherung (engl. generalized gradient approximation, GGA) von Perdew, Burke und Enzerhof (PBE) [110] zum Einsatz. Lediglich für Cadmium wurde das Austauschpotential von Wu und Cohen [111] verwendet. Die verwendeten Radien $R_{\mathrm{MT}}$ (engl. muffin tin radius), innerhalb derer Kugelflächenfunktionen statt ebener Wellen verwendet werden; die Energie $E_{\text {sep }}$, die zwischen Kern- und Valenzzuständen unterscheidet; die Größe des Basissatzes $K_{\max }$, die maximale Quantenzahl $l_{\max }$ der Wellenfunktion innerhalb des muffin-tin-Radius sowie die Anzahl der (unverschobenen) k-Punkte in der Brillouin-Zone sind in den Tabellen A.1 und A.2 systemspezifisch angegeben. Die Fourierentwicklung der Ladungsdichte wurde jeweils bis zu einem Betrag der Basisvektoren von $G_{\max }=16 \sqrt{\mathrm{Ry}}$ bestimmt.

Jedes System wurde im Grundzustand betrachtet, weswegen jeweils zu Beginn der Rechnung jenes Volumen und $c / a$-Verhältnis der Einheitszelle gesucht wurde, das die Energie des Gesamtsystems minimiert. Dies geschah ausgehend von experimentellen Gitterparametern und den durch die Raumgruppe vorgegebenen Atompositionen. Im Falle der MAXPhasen sind die Atompositionen nicht vollständig durch die Raumgruppe bestimmt, der hierbei zusätzlich auftretende Freiheitsgrad wurde simultan optimiert. Alle weiteren Rechnungen wurden mit den so gewonnenen optimierten Gitterkonstanten $a_{0}$ und $c_{0}$ durchgeführt. Zur Berechnung des EFGs an einer bestimmten Sonde wurde, wenn es sich bei der Sonde um ein Fremdatom handelte, eine ungefähr kubische Superzelle aus der Elementarzelle gebildet, in der die Sonde substitutionell eingebracht wurde.

Tabelle A.1: Details der DFT-Rechnungen kubischer Systeme.

\begin{tabular}{|c|c|c|c|c|c|c|}
\hline $\begin{array}{l}\text { Wirtsgitter } \\
\text { Raumgruppe } \\
\text { Sondenatom } \\
\text { Punktgruppe }\end{array}$ & $\begin{array}{c}\mathrm{Al} \\
F m \overline{3} m \\
\mathrm{Al} \\
O_{h}-m \overline{3} m\end{array}$ & $\begin{array}{c}\mathrm{Au} \\
F m \overline{3} m \\
\mathrm{Au} \\
O_{h}-m \overline{3} m\end{array}$ & $\begin{array}{c}\mathrm{Cu} \\
F m \overline{3} m \\
\mathrm{Cu} \\
O_{h}-m \overline{3} m\end{array}$ & $\begin{array}{c}\text { Mo } \\
\text { Im } m \overline{3} m \\
\text { Mo } \\
O_{h}-m \overline{3} m\end{array}$ & $\begin{array}{c}\mathrm{Nb} \\
\operatorname{Im} \overline{3} m \\
\mathrm{Nb} \\
O_{h}-m \overline{3} m\end{array}$ & $\begin{array}{c}\text { Ta } \\
I m \overline{3} m \\
\mathrm{Ta} \\
O_{h}-m \overline{3} m\end{array}$ \\
\hline$[\AA]$ & 4,0445 & 4,1535 & 3,6315 & 3,1689 & 3,3129 & 3,3201 \\
\hline [a.u.] & $\mathrm{Al}: 2,5$ & Au: 2,5 & $\mathrm{Cu}: 2,41$ & Mo: 2,5 & $\mathrm{Nb}: 2,5$ & Ta: 2,5 \\
\hline$E_{\text {sep }} \quad[\mathrm{Ry}]$ & -8 & -8 & -9 & -6 & -6 & -6 \\
\hline$K_{\max } \cdot R_{\mathrm{MT}}^{\min }$ & 7 & 8,5 & 8 & 8 & 8 & 8 \\
\hline$l_{\max }$ & 10 & 10 & 10 & 10 & 10 & 10 \\
\hline k-Punkte & 24000 & 24000 & 24000 & 24000 & 24000 & 24000 \\
\hline
\end{tabular}


Tabelle A.2: Details der DFT-Rechnungen in Cd-dotieren hexagonalen Metallen und MAX-Phasen.

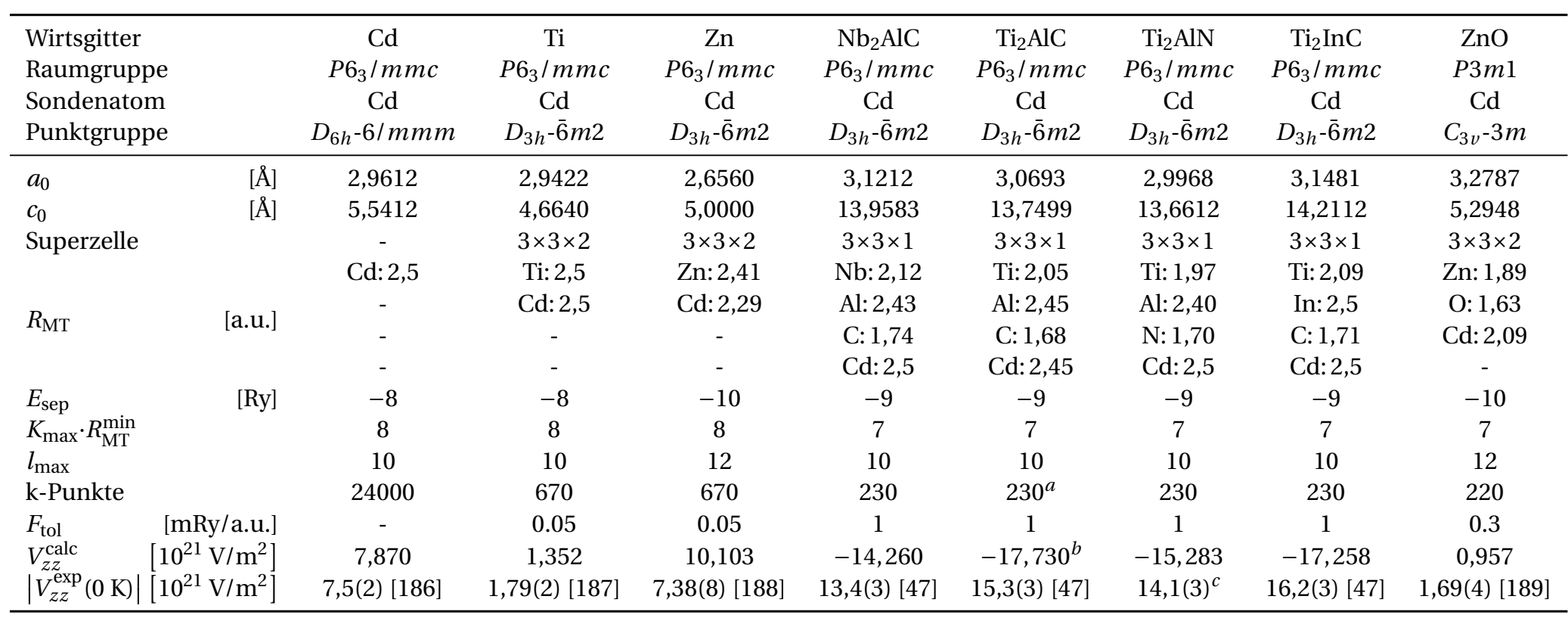

$a_{300}$ k-Punkte für die orthorhombische Dehnung.

${ }^{b}$ Ladungskonvergenzkriterium von $10^{-5} e$.

${ }^{c}$ Diese Arbeit. 


\section{Anhang B}

\section{Dehnungsfelder um Versetzungen}

Der Dehnungstensor $\varepsilon_{i j}(x, z)$ um eine Stufenversetzung in der Basalebene eines hexagonalen Kristalls ist mit Burgersvektor $b$ entlang der $x$-Achse, der Versetzungslinie entlang der $y$-Achse und kristallographischer $c$-Achse entlang der $z$-Achse durch

$$
\begin{aligned}
& \varepsilon_{x x}(x, z)=E(x, z) \cdot\left[\left(\overline{c_{11}}+c_{13}+c_{44}\right) x^{2} z+c_{44} \lambda^{2} z^{3}\right], \\
& \varepsilon_{z z}(x, z)=-\frac{E(x, z)}{c_{33}} \cdot\left[\left(c_{13}^{2}+\overline{c_{11}} c_{13}+2 c_{13} c_{44}+\overline{c_{11}} c_{44}\right) x^{2} z-\overline{c_{11}} c_{44} \lambda^{2} z^{3}\right], \\
& \varepsilon_{x z}(x, z)=-\frac{E(x, z)}{2} \cdot\left(c_{13}+\overline{c_{11}}\right) \cdot\left(x^{3}-\lambda^{2} x z^{2}\right)
\end{aligned}
$$

gegeben, wobei $c_{m n}$ die elastischen Konstanten sind und für

$$
\begin{aligned}
E(x, z) & =\frac{-b \lambda\left(c_{13}-\overline{c_{11}}\right)}{4 \pi q(x, z)^{2} t(x, z)^{2} \overline{c_{11}} c_{44} \sin \Phi}, \\
q(x, z)^{2} & =x^{2}+2 x z \lambda \cos \Phi+z^{2} \lambda^{2}, \\
t(x, z)^{2} & =x^{2}-2 x z \lambda \cos \Phi+z^{2} \lambda^{2}, \\
\lambda & =\sqrt[4]{c_{11} / c_{33}}, \\
\overline{c_{11}} & =\sqrt{c_{11} c_{33}}, \\
\Phi & =\frac{1}{2} \arccos \frac{c_{13}^{2}+2 c_{13} c_{44}-{\overline{c_{11}}}^{2}}{2 \overline{c_{11}} c_{44}}
\end{aligned}
$$

gilt [54]. Der Versetzungskern liegt bei $x=0$ und $z=0$.

Der Dehnungstensor $\varepsilon_{i j}(x, z)$ um eine Schraubenversetzung in der Basalebene eines hexagonalen Kristalls ist mit Burgersvektor und Versetzungslinie entlang der $y$-Achse sowie kristallographischer $c$-Achse entlang der $z$-Achse durch

$$
\begin{aligned}
& \varepsilon_{y z}(x, z)=-\frac{b}{4 \pi} \cdot \frac{\sqrt{c_{44} c_{66}}}{c_{44} x^{2}+c_{66} z^{2}} \cdot x, \\
& \varepsilon_{x y}(x, z)=\frac{b}{4 \pi} \cdot \frac{\sqrt{c_{44} c_{66}}}{c_{44} x^{2}+c_{66} z^{2}} \cdot z
\end{aligned}
$$

gegeben [54]. 



\section{Literaturverzeichnis}

[1] E. Rudy und H. Nowotny. Untersuchungen im System Hafnium-Tantal-Kohlenstoff. Monatsh. Chem. 94(3):507-517, 1963.

[2] C. E. Guillaume. The anomaly of the nickel-steels. Proc. Phys. Soc. London 32(12):374, 1920.

[3] J. G. Bednorz und K. A. Müller. Possible high $\mathrm{T}_{c}$ superconductivity in the Ba-La-Cu-O system. Z. Phys. B: Condens. Matter 64(2):189-193, 1986.

[4] W. Jeitschko, H. Nowotny und F. Benesovsky. Kohlenstoffhaltige ternäre Verbindungen (H-Phase). Monatsh. Chem. 94(4):672-676, 1963.

[5] W. Jeitschko, H. Nowotny und F. Benesovsky. $\mathrm{Ti}_{2} \mathrm{AlN}$, eine stickstoffhaltige H-Phase. Monatsh. Chem. 94(6):1198-1200, 1963.

[6] W. Jeitschko, H. Nowotny und F. Benesovsky. Die H-Phasen $\mathrm{Ti}_{2} \mathrm{InC}, \mathrm{Zr}_{2} \mathrm{InC}, \mathrm{Hf}_{2} \mathrm{InC}$ und $\mathrm{Ti}_{2}$ GeC. Monatsh. Chem. 94(6):1201-1205, 1963.

[7] W. Jeitschko, H. Nowotny und F. Benesovsky. Die H-Phasen $\mathrm{Ti}_{2} \mathrm{TlC}, \mathrm{Ti}_{2} \mathrm{PbC}, \mathrm{Nb}_{2} \mathrm{InC}$, $\mathrm{Nb}_{2} \mathrm{SnC}$ und $\mathrm{Ta}_{2} \mathrm{GaC}$. Monatsh. Chem. 95(2):431-435, 1964.

[8] H. Nowotny. Strukturchemie einiger Verbindungen der Übergangsmetalle mit den Elementen C, Si, Ge, Sn. Prog. Solid State Chem. 5:27-70, 1971.

[9] M. W. Barsoum, L. Farber, I. Levin, A. Procopio, T. El-Raghy und A. Berner. Highresolution transmission electron microscopy of $\mathrm{Ti}_{4} \mathrm{AlN}_{3}$, or $\mathrm{Ti}_{3} \mathrm{Al}_{2} \mathrm{~N}_{2}$ revisited. J. Am. Ceram. Soc. 82(9):2545-2547, 1999.

[10] W. Jeitschko und H. Nowotny. Die Kristallstruktur von $\mathrm{Ti}_{3} \mathrm{SiC}_{2}$ - ein neuer Komplexcarbid-Typ. Monatsh. Chem. 98(2):329-337, 1967.

[11] A. S. Ingason, A. Petruhins, M. Dahlqvist, F. Magnus, A. Mockute, B. Alling, L. Hultman, I. A. Abrikosov, P. O. A. Persson und J. Rosen. A nanolaminated magnetic phase: $\mathrm{Mn}_{2}$ GaC. Mat. Res. Lett. 2(2):89-93, 2014.

[12] M. Naguib, G. W. Bentzel, J. Shah, J. Halim, E. N. Caspi, J. Lu, L. Hultman und M. W. Barsoum. New Solid Solution MAX Phases: $\left(\mathrm{Ti}_{0.5}, \mathrm{~V}_{0.5}\right)_{3} \mathrm{AlC}_{2},\left(\mathrm{Nb}_{0.5}, \mathrm{~V}_{0.5}\right){ }_{2} \mathrm{AlC}$, $\left(\mathrm{Nb}_{0.5}, \mathrm{~V}_{0.5}\right)_{4} \mathrm{AlC}_{3}$ and $\left(\mathrm{Nb}_{0.8}, \mathrm{Zr}_{0.2}\right)_{2}$ AlC. Mat. Res. Lett. 2(4):233-240, 2014.

[13] M. W. Barsoum. MAX Phases: Properties of machinable ternary carbides and nitrides Wiley-VCH Weinheim, 2013. 


\section{Literaturverzeichnis}

[14] J. Etzkorn, M. Ade und H. Hillebrecht. $\mathrm{Ta}_{3} \mathrm{AlC}_{2}$ and $\mathrm{Ta}_{4} \mathrm{AlC}_{3}$-single-crystal investigations of two new ternary carbides of tantalum synthesized by the molten metal technique. Inorg. Chem. 46(4):1410-1418, 2007.

[15] P. Eklund, M. Beckers, U. Jansson, H. Högberg und L. Hultman. The $\mathrm{M}_{n+1} \mathrm{AX}$ 的 ses: Materials science and thin-film processing. Thin Solid Films 518(8):1851 - 1878, 2010.

[16] V. Vishnyakov, O. Crisan, P. Dobrosz und J. S. Colligon. Ion sputter-deposition and in-air crystallisation of $\mathrm{Cr}_{2} \mathrm{AlC}$ films. Vacuum 100:61 - 65, 2014.

[17] M. W. Barsoum und T. El-Raghy. Synthesis and characterization of a remarkable ceramic: $\mathrm{Ti}_{3} \mathrm{SiC}_{2}$. J. Am. Ceram. Soc. 79(7):1953-1956, 1996.

[18] M. W. Barsoum, T. Zhen, S. R. Kalidindi, M. Radovic und A. Murugaiah. Fully reversible, dislocation-based compressive deformation of $\mathrm{Ti}_{3} \mathrm{SiC}_{2}$ to $1 \mathrm{GPa}$. Nat. $\mathrm{Ma}$ ter. 2(2):107-111, 2003.

[19] N. G. Jones, C. Humphrey, L. D. Connor, O. Wilhelmsson, L. Hultman, H. J. Stone, F. Giuliani und W. J. Clegg. On the relevance of kinking to reversible hysteresis in MAX phases. Acta Mater. 69:149-161, 2014.

[20] K. Sakamaki, H. Wada, H. Nozaki, Y. Ōnuki und M. Kawai. Carbosulfide superconductor. Solid State Commun. 112(6):323-327, 1999.

[21] D. J. Tallman, B. Anasori und M. W. Barsoum. A critical review of the oxidation of $\mathrm{Ti}_{2} \mathrm{AlC}, \mathrm{Ti}_{3} \mathrm{AlC}_{2}$ and $\mathrm{Cr}_{2} \mathrm{AlC}$ in air. Mat. Res. Lett. 1(3):115-125, 2013.

[22] M. Sundberg, G. Malmqvist, A. Magnusson und T. El-Raghy. Alumina forming high temperature silicides and carbides. Ceram. Int. 30(7):1899-1904, 2004.

[23] S. Gupta, D. Filimonov, T. Palanisamy, T. El-Raghy und M. W. Barsoum. Ta ${ }_{2} \mathrm{AlC}$ and $\mathrm{Cr}_{2} \mathrm{AlC}$ Ag-based composites-new solid lubricant materials for use over a wide temperature range against Ni-based superalloys and alumina. Wear 262(11):1479-1489, 2007.

[24] M. W. Barsoum. The $\mathrm{M}_{n+1} \mathrm{AX}_{n}$ phases: A new class of solids: Thermodynamically stable nanolaminates. Prog. Solid State Chem. 28(1-4):201-281, 2000.

[25] E. N. Hoffman, D. W. Vinson, R. L. Sindelar, D. J. Tallman, G. Kohse und M. W. Barsoum. MAX phase carbides and nitrides: Properties for future nuclear power plant in-core applications and neutron transmutation analysis. Nucl. Eng. Des. 244:17-24, 2012.

[26] R. Grieseler, B. Hähnlein, M. Stubenrauch, T. Kups, M. Wilke, M. Hopfeld, J. Pezoldt und P. Schaaf. Nanostructured plasma etched, magnetron sputtered nanolaminar $\mathrm{Cr}_{2} \mathrm{AlC}$ MAX phase thin films. Appl. Surf. Sci. 292:997-1001, 2014. 
[27] B. Pécz, L. Tóth, M. A. di Forte-Poisson und J. Vacas. $\mathrm{Ti}_{3} \mathrm{SiC}_{2}$ formed in annealed $\mathrm{Al} / \mathrm{Ti}$ contacts to p-type SiC. Appl. Surf. Sci 206(1):8-11, 2003.

[28] M. R. Lukatskaya, O. Mashtalir, C. E. Ren, Y. Dall'Agnese, P. Rozier, P. L. Taberna, M. Naguib, P. Simon, M. W. Barsoum und Y. Gogotsi. Cation intercalation and high volumetric capacitance of two-dimensional titanium carbide. Science 341(6153):1502-1505, 2013.

[29] J. Halim, M. R. Lukatskaya, K. M. Cook, J. Lu, C. R. Smith, L.-Å. Naslund, S. J. May, L. Hultman, Y. Gogotsi, P. Eklund und M. W. Barsoum. Transparent conductive twodimensional titanium carbide epitaxial thin films. Chem. Mater. 26(7):2374-2381, 2014.

[30] L. Farber, M. W. Barsoum, A. Zavaliangos, T. El-Raghy und I. Levin. Dislocations and stacking faults in $\mathrm{Ti}_{3} \mathrm{SiC}_{2}$. J. Am. Ceram. Soc. 81(6):1677-1681, 1998.

[31] M. W. Barsoum und T. El-Raghy. Room-temperature ductile carbides. Metall. Mater. Trans. A 30(2):363-369, 1999.

[32] B. J. Kooi, R. J. Poppen, N. J. M. Carvalho, J. T. M. De Hosson und M. W. Barsoum. $\mathrm{Ti}_{3} \mathrm{SiC}_{2}$ : A damage tolerant ceramic studied with nano-indentations and transmission electron microscopy. Acta Mat. 51(10):2859-2872, 2003.

[33] M.-C. Maaß. In-situ TEM Verformung von $\mathrm{Ti}_{3} \mathrm{SiC}_{2}$ - MAX Phase. Masterarbeit, Georg-August-Universität Göttingen, 2014.

[34] I. Salama, T. El-Raghy und M. W. Barsoum. Synthesis and mechanical properties of $\mathrm{Nb}_{2} \mathrm{AlC}$ and (Ti,Nb) ${ }_{2}$ AlC. J. Alloys Compd. 347(1-2):271-278, 2002.

[35] J.C. Schuster, H. Nowotny und C. Vaccaro. The ternary systems: CrAlC, VAlC, and TiAlC and the behavior of H-phases (M2AlC). J. Solid State Chem. 32(2):213-219, 1980 .

[36] M. W. Barsoum, M. Ali und T. El-Raghy. Processing and characterization of $\mathrm{Ti}_{2} \mathrm{AlC}$, $\mathrm{Ti}_{2} \mathrm{AlN}$, and $\mathrm{Ti}_{2} \mathrm{AlC}_{0.5} \mathrm{~N}_{0.5}$. Metall. Mater. Trans. A31(7):1857-1865, 2000.

[37] C. Brüsewitz, I. Knorr, H. Hofsäss, M. W. Barsoum und C. A. Volkert. Single crystal pillar microcompression tests of the MAX phases $\mathrm{Ti}_{2} \mathrm{InC}$ and $\mathrm{Ti}_{4} \mathrm{AlN}_{3}$. Scr. Mater. 69(4):303-306, 2013.

[38] C. Tromas, P. Villechaise, V. Gauthier-Brunet und S. Dubois. Slip line analysis around nanoindentation imprints in $\mathrm{Ti}_{3} \mathrm{SnC}_{2}$ : a new insight into plasticity of MAX-phase materials. Philos. Mag. 91(7-9):1265-1275, 2011.

[39] J. M. Molina-Aldareguia, J. Emmerlich, J. P. Palmquist, U. Jansson und L. Hultman. Kink formation around indents in laminated $\mathrm{Ti}_{3} \mathrm{SiC}_{2}$ thin films studied in the nanoscale. Scr. Mater. 49(2):155-160, 2003. 


\section{Literaturverzeichnis}

[40] M. F. Cover, O. Warschkow, M. M. M. Bilek und D. R. McKenzie. A comprehensive survey of $\mathrm{M}_{2} \mathrm{AX}$ phase elastic properties. J. Phys. Condens. Matter 21(30):305403, 2009.

[41] X. He, Y. Bai, C. Zhu und M. W. Barsoum. Polymorphism of newly discovered $\mathrm{Ti}_{4} \mathrm{GaC}_{3}$ : A first-principles study. Acta Mat. 59(14):5523-5533, 2011.

[42] X. He, Y. Bai, Y. Chen, C. Zhu, M. Li und M. W. Barsoum. Phase stability, electronic structure, compressibility, elastic and optical properties of a newly discovered $\mathrm{Ti}_{3} \mathrm{SnC}_{2}$ : A first-principle study. J. Am. Ceram. Soc. 94(11):3907-3914, 2011.

[43] H. Frauenfelder und R. M. Steffen. Alpha-, beta- and gamma-ray spectroscopy, Seiten 997-1198. North-Holland Publishing Company, 1965.

[44] D. Jürgens, M. Uhrmacher, H. Hofsäss, J. Röder, P. Wodniecki, A. Kulinska und M. W. Barsoum. First PAC experiments in MAX-phases. Hyperfine Interact. 178(1-3):23-30, 2007.

[45] D. Jürgens, M. Uhrmacher, H. Hofsäss, J. Mestnik-Filho und M. W. Barsoum. Perturbed angular correlation studies of the MAX phases $\mathrm{Ti}_{2} \mathrm{AlN}$ and $\mathrm{Cr}_{2} \mathrm{GeC}$ using ion implanted ${ }^{111}$ In as probe nuclei. Nucl. Instrum. Methods Phys. Res., Sect. B 268(1112):2185-2188, 2010.

[46] D. Jürgens, M. Uhrmacher, H. G. Gehrke, M. Nagl, U. Vetter, C. Brüsewitz, H. Hofsäss, J. Mestnik-Filho und M. W. Barsoum. Electric field gradients at ${ }^{111} \mathrm{In} /{ }^{111} \mathrm{Cd}$ probe atoms on A-sites in 211-MAX phases. J. Phys. Condens. Matter 23(50):505501, 2011.

[47] D. Jürgens. Untersuchung der elektrischen Hyperfeinwechselwirkung in $M_{n+1} A X_{n}$ Phasen mittels der gestörten $\gamma-\gamma$-Winkelkorrelation Doktorarbeit, Universität Göttingen, 2014.

[48] R. G. Shulman, B. J. Wyluda und P. W. Anderson. Nuclear magnetic resonance in semiconductors. II. Quadrupole broadening of nuclear magnetic resonance lines by elastic axial deformation. Phys. Rev. 107(4):953-958, 1957.

[49] W. Yu, S. Li und W. G. Sloof. Microstructure and mechanical properties of a $\mathrm{Cr}_{2} \mathrm{Al}(\mathrm{Si}) \mathrm{C}$ solid solution. Mat. Sci. Eng. A 527(21):5997-6001, 2010.

[50] B. Manoun, S. K. Saxena, G. Hug, A. Ganguly, E. N. Hoffman und M. W. Barsoum. Synthesis and compressibility of $\mathrm{Ti}_{3}\left(\mathrm{Al}_{1} \mathrm{Sn}_{0.2}\right) \mathrm{C}_{2}$ and $\mathrm{Ti}_{3} \mathrm{Al}\left(\mathrm{C}_{0.5}, \mathrm{~N}_{0.5}\right)_{2}$. J. Appl. Phys. 101(11):113523, 2007.

[51] W. Voigt. Lehrbuch der Kristallphysik BG Teubner, 1910.

[52] J. F. Nye. Physical properties of crystals: their representation by tensors and matrices. Clarendon Press, 1957.

[53] J. D. Eshelby. The continuum theory of lattice defects. Solid state physics 3:79-144, 1956. 
[54] J. P. Hirth und J. Lothe. Theory of dislocations. John Wiley \& Sons New York, 1982.

[55] C. W. Garland und J. Silverman. Elastic constants of cadmium from $4.2 \mathrm{~K}$ to $300 \mathrm{~K}$. Phys. Rev. 119(4):1218-1222, 1960.

[56] E. S. Fisher und C. J. Renken. Single-crystal elastic moduli and the hcp-bcc transformation in Ti, Zr, and Hf. Phys. Rev. 135(2A):A482-A494, 1964.

[57] H. M. Ledbetter. Elastic properties of zinc: A compilation and a review. J. Phys. Chem. Ref. Data 6(4):1181-1203, 1977.

[58] T. B. Bateman. Elastic moduli of single-crystal zinc oxide. J. Appl. Phys. 33(11):33093312, 1962.

[59] P. M. Sutton. The variation of the elastic constants of crystalline aluminum with temperature between 63 K and 773 K. Phys. Rev.91(6):816-821, 1953.

[60] J. R. Neighbours und G. A. Alers. Elastic constants of silver and gold. Phys. Rev. 111(3):707-712, 1958.

[61] W. C. Overton und J. Gaffney. Temperature variation of the elastic constants of cubic elements. I. Copper. Phys. Rev. 98(4):969-977, 1955.

[62] F. H. Featherston und J. R. Neighbours. Elastic constants of tantalum, tungsten, and molybdenum. Phys. Rev. 130(4):1324-1333, 1963.

[63] K. J. Carroll. Elastic constants of niobium from $4.2 \mathrm{~K}$ to $300 \mathrm{~K}$. J. Appl. Phys. 36(11):3689-3690, 1965.

[64] M. W. Barsoum, L. Farber und T. El-Raghy. Dislocations, kink bands, and roomtemperature plasticity of $\mathrm{Ti}_{3} \mathrm{SiC}_{2}$. Metall. Mater. Trans. A 30(7):1727-1738, 1999.

[65] L. Farber, I. Levin und M. W. Barsoum. Transmission electron microscopy study of a low-angle boundary in plastically deformed $\mathrm{Ti}_{3} \mathrm{SiC}_{2}$. Philos. Mag. Lett. 79(4):163-170, 1999.

[66] F. C. Frank und A. N. Stroh. On the theory of kinking. Proc. Phys. Soc. London, Sect. $B$ 65(10):811, 1952.

[67] G. Gottstein. Physikalische Grundlagen der Materialkunde Springer Berlin Heidelberg, 2007.

[68] M. W. Barsoum und S. Basu. Kinking nonlinear elastic solids. In Encyclopedia of Materials: Science and Technology (Second Edition) Elsevier, 2010.

[69] J. Christiansen, P. Heubes, R. Keitel, W. Klinger, W. Loeffler, W. Sandner und W. Witthuhn. Temperature dependence of the electric field gradient in noncubic metals. $Z$. Physik B 24(2):177-187, 1976. 


\section{Literaturverzeichnis}

[70] M. Forker und S. Scholz. Linear temperature dependence of the electric quadrupole interaction of ${ }^{111} \mathrm{Cd}$ impurities in holmium. Hyperfine Interact. 7(1):353-360, 1979.

[71] P. Wodniecki, B. Wodniecka, A. Kulińska, M. Uhrmacher und K.-P. Lieb. The electric field gradients in $(\mathrm{Zr} / \mathrm{Hf}) \mathrm{Al}_{3}$ and $(\mathrm{Zr} / \mathrm{Hf})_{2} \mathrm{Al}_{3}$ intermetallic compounds studied by ${ }^{181} \mathrm{Ta}$ - and ${ }^{111} \mathrm{Cd}-\mathrm{PAC}$ spectroscopy. Hyperfine interact. 136(3-8):535-539, 2001.

[72] D. Torumba, K. Parlinski, M. Rots und S. Cottenier. Temperature dependence of the electric-field gradient in hcp-Cd from first principles. Phys. Rev. B 74(14):144304, 2006.

[73] F. E. Neumann und O. E. Meyer. Vorlesungen über die Theorie der Elasticität der festen Körper und des Lichtäthers: gehalten an der Universität Königsberg. BG Teubner, 1885.

[74] F. G. Fumi. Physical properties of crystals: The direct-inspection method. Acta Crystallogr. 5(1):44-48, 1952.

[75] F. G. Fumi. Matter tensors in symmetrical systems. Il Nuovo Cimento 9(9):739-756, 1952.

[76] E. F. Taylor und N. Bloembergen. Nuclear spin saturation by ultrasonics in sodium chloride. Phys. Rev. 113(2):431, 1959.

[77] R. K. Sundfors. Nuclear acoustic resonance of ${ }^{73} \mathrm{Ge}$ in single-crystal germanium; interpretation of experimental gradient-elastic-tensor components in germanium and zinc-blende compounds. Phys. Rev. B 20(9):3562, 1979.

[78] R. K. Sundfors. Experimental gradient-elastic tensors and chemical bonding in III-V semiconductors. Phys. Rev. B 10(10):4244-4252, 1974.

[79] O. Kanert. Quadrupole broadening of nuclear magnetic resonance lines in deformed copper and aluminum. Phys. Status Solidi B 32(2):667-677, 1969.

[80] O. Kanert und K. Preusser. X-ray and nuclear magnetic resonance investigations on deformed aluminium single crystals. Phys. Status Solidi A 15(2):483-489, 1973.

[81] J. Buttet. Determination of the gradient-elastic tensor in aluminium using nuclear acoustic resonance. J. Phys. F: Met. Phys. 3(5):918, 1973.

[82] O. Kanert und K. Preusser. Gradient-elastic tensor in aluminium. Solid State Commun. 15(1):97 - 99, 1974.

[83] R. K. Sundfors, T. H. Wang, D. I. Bolef, P. A. Fedders und D. G. Westlake. Nuclear acoustic resonance in single-crystal hydrogen-free tantalum. Phys. Rev. B 12(1):2630, 1975.

[84] J. Pellisson und J. Buttet. Nuclear-acoustic-resonance determination of the gradientelastic tensor and indirect nuclear-spin interactions in niobium. Phys. Rev. B 11(1):48-59, 1975. 
[85] E. Fischer, V. Müller, D. Ploumbidis und G. Schanz. Nuclear-acoustic-resonance determination of the electronic contribution to the electric-field-gradient tensor in molybdenum. Phys. Rev. Lett. 40(12):796-799, 1978.

[86] B. Ströbel und V. Müller. Nuclear-acoustic-resonance determination of the strain electric-field-gradient tensor $S$ and its temperature dependence in Ta single crystals. Phys. Rev. B 24(11):6292-6303, 1981.

[87] R. K. Sundfors. Nuclear acoustic resonance in single-crystal gold metal. Phys. Rev. B 28(4):2284-2286, 1983.

[88] R. Przewodnik, P. Kessler und R. Vianden. Hyperfine fields in $\mathrm{ZnO}$ studied under uniand biaxial pressure. Hyperfine Interact. 221(1-3):111-116, 2013.

[89] T. Butz. On the volume and structure dependence of electric field gradients in closepacked metals. I. Phys. Scr. 17(2):87, 1978.

[90] J. W. Zwanziger, U. Werner-Zwanziger, J. L. Shaw und C. So. Stress, strain, and NMR. Solid State Nucl. Magn. Reson. 29(1-3):113-118, 2006.

[91] O. Kanert und M. Mehring. Static quadrupole effects in disordered cubic solids. In NMR Springer Berlin Heidelberg, 1971.

[92] B. Wodniecka, M. Marszalek und P. Wodniecki. TDPAC studies on the recovery of Ag plastically deformed at room temperature. J. Phys. Condens. Matter 1(41):7521, 1989.

[93] S. V. Verkhovskii, A. Yu. Yakubovsky, B. Z. Malkin, S. K. Saikin, M. Cardona, A. Trokiner und V. I. Ozhogin. Isotopic disorder in Ge single crystals probed with ${ }^{73} \mathrm{Ge}$ NMR. Phys. Rev. B68(10):104201, 2003.

[94] E. A. Chekhovich, K. V. Kavokin, J. Puebla, A. B. Krysa, M. Hopkinson, A. D. Andreev, A. M. Sanchez, R. Beanland, M. S. Skolnick und A. I. Tartakovskii. Structural analysis of strained quantum dots using nuclear magnetic resonance. Nat. Nanotechnol. 7(10):646-650, 2012.

[95] C. Bulutay. Quadrupolar spectra of nuclear spins in strained $\operatorname{In}_{x} \mathrm{Ga}_{1-x}$ As quantum dots. Phys. Rev. B 85(11):115313, 2012.

[96] M. S. Kuznetsova, K. Flisinski, I. Ya. Gerlovin, M. Yu. Petrov, I. V. Ignatiev, S. Yu. Verbin, D. R. Yakovlev, D. Reuter, A. D. Wieck und M. Bayer. Nuclear magnetic resonances in (In,Ga)As/GaAs quantum dots studied by resonant optical pumping. Phys. Rev. B 89(11):125304, 2014.

[97] T. Butz und H. Ernst. The electric field gradient in $\mathrm{Cd}_{1-x} \mathrm{Mg}_{x}$ for $0<\mathrm{x}<0.15$ measured by perturbed angular correlations. Phys. Lett. A 53(5):387 - 389, 1975.

[98] S. Unterricker, H. J. Hunger und J. Hausbrand. The concentration dependence of the quadrupole interaction in the disordered system $\mathrm{Mg}_{x} \mathrm{Cd}_{1-x}$ at high temperatures. Phys. Status Solidi B 69(2):K111-K115, 1975. 


\section{Literaturverzeichnis}

[99] P. Raghavan, R. S. Raghavan und W. B. Holzapfel. Effect of high pressure on the quadrupole interaction in Cd metal measured by perturbed angular correlations. Phys. Rev. Lett. 28(14):903-906, 1972.

[100] T. Butz, B. Lindgren und H. Saitovitch. The effect of lattice vibrations on the pressure dependence of the electric field gradient in cadmium metal. Hyperfine Interact. 7(1):81-91, 1979.

[101] L. A. Terrazos, H. M. Petrilli, M. Marszalek, H. Saitovitch, P. R. J. Silva, P. Blaha und K. Schwarz. Electric field gradients at $\mathrm{Ta}$ in $\mathrm{Zr}$ and $\mathrm{Hf}$ inter-metallic compounds. Solid State Commun. 121(9-10):525 - 529, 2002.

[102] P Wodniecki, A Kulińska, B Wodniecka, J Belošević-Čavor, V Koteski und M Uhrmacher. Electric field gradients at $\mathrm{Ta}$ and $\mathrm{Cd}$ in $\mathrm{Ti}_{2} \mathrm{Pd}$ compound . J. Alloys Compd. 477(1-2):36 - 39, 2009.

[103] G. E. Peterson, J. R. Carruthers und A. Carnevale. ${ }^{93} \mathrm{Nb}$ NMR Study of the $\mathrm{LiNbO}_{3}-$ $\mathrm{LiTaO}_{3}$ solid-solution system. J. Chem. Phys. 53(6):2436-2442, 1970.

[104] J Seliger und V Žagar. Nuclear-quadrupole double-resonance study of the solid solution $\mathrm{Rb}_{1-x} \mathrm{Tl}_{x} \mathrm{H}_{2} \mathrm{PO}_{4}$. Phys. Rev. B 52(2):1070, 1995.

[105] R. L. Rasera, R. C. Reno, G. Schmidt, T. Butz, A. Vasquez, H. Ernst, G. K. Shenoy und B. D. Dunlap. Strength, symmetry and distribution of electric quadrupole interactions at ${ }^{181}$ Ta impurities in hafnium-zirconium alloys. J. Phys. F: Met. Phys. 8(7):1579, 1978.

[106] C. Budtz-Jrgensen und K. Bonde Nielsen. Perturbed angular correlations with 111Cd in the $\alpha$ and $\delta$ phases of In-Sn. Hyperfine Interact. 1(1):81-92, 1975.

[107] S. Cottenier. Density functional theory and the family of (L)APW-methods: a stepby-step introduction 2002.

[108] P. Hohenberg und W. Kohn. Inhomogeneous electron gas. Phys. Rev. 136:864-871, 1964.

[109] W. Kohn und L. J. Sham. Self-consistent equations including exchange and correlation effects. Phys. Rev. 140(4A):1133-1138, 1965.

[110] J. P. Perdew, K. Burke und M. Ernzerhof. Generalized gradient approximation made simple. Phys. Rev. Lett.77(18):3865-3868, 1996.

[111] Z. Wu und R. E. Cohen. More accurate generalized gradient approximation for solids. Phys. Rev. B 73(23):235116, 2006.

[112] W. E. Pickett. Pseudopotential methods in condensed matter applications. Comput. Phys. Rep. 9(3):115-197, 1989.

[113] J. C. Slater. Wave functions in a periodic potential. Phys. Rev. 51(10):846, 1937. 
[114] O. K. Andersen. Linear methods in band theory. Phys. Rev. B 12(8):3060, 1975.

[115] E. Sjöstedt, L. Nordström und D. J. Singh. An alternative way of linearizing the augmented plane-wave method. Solid State Commun. 114(1):15-20, 2000.

[116] G. K. H. Madsen, P. Blaha, K. Schwarz, E. Sjöstedt und L. Nordström. Efficient linearization of the augmented plane-wave method. Phys. Rev. B 64(19):195134, 2001.

[117] K. Schwarz, P. Blaha und G. K. H. Madsen. Electronic structure calculations of solids using the WIEN2k package for material sciences. Comput. Phys. Commun. 147(12):71-76, 2002.

[118] P. Blaha, K. Schwarz, G. K. H. Madsen, D. Kvasnicka und J. Luitz. WIEN2k. An augmented plane wave plus local orbitals program for calculating crystal properties 2001.

[119] D. Singh. Ground-state properties of lanthanum: Treatment of extended-core states. Phys. Rev. B 43(8):6388, 1991.

[120] H. Haas und J. Correia. The quadrupole moments of $\mathrm{Zn}$ and Cd isotopes - an update. Hyperfine Interact. 198(1-3):133-137, 2010.

[121] Y. Abreu, C. M. Cruz, P. Van Espen, C. Pérez, I. Piñera, A. Leyva und A. E. Cabal. Electric field gradient calculations in $\mathrm{ZnO}$ samples implanted with ${ }^{111} \operatorname{In}\left({ }^{111} \mathrm{Cd}\right)$. Solid State Commun. 152(5):399-402, 2012.

[122] P. Blaha, K. Schwarz und P. H. Dederichs. First-principles calculation of the electricfield gradient in hcp metals. Phys. Rev. B 37(6):2792-2796, 1988.

[123] D. Torumba, V. Vanhoof, M. Rots und S. Cottenier. Hyperfine interactions at lanthanide impurities in Fe. Phys. Rev. B 74(1):014409, 2006.

[124] J. A. H. da Jornada und F. C. Zawislak. Effects of high pressure on the electric field gradient in $s p$ metals. Phys. Rev. B 20(7):2617-2623, 1979.

[125] G. Schatz und A. Weidinger. Nukleare Festkörperphysik. Teubner Verlag, 1992.

[126] D. R. Hamilton. On directional correlation of successive quanta. Phys. Rev. 58(2):122$131,1940$.

[127] E. M. Purcell, H. C. Torrey und R. V. Pound. Resonance absorption by nuclear magnetic moments in a solid. Phys. Rev. 69(1-2):37, 1946.

[128] F. Bloch. Nuclear induction. Phys. Rev. 70(7-8):460, 1946.

[129] C. P. Slichter. Principles of magnetic resonance Springer Verlag Berlin Heidelberg, 1990.

[130] R. L. Mössbauer. Kernresonanzfluoreszenz von Gammastrahlung in $\operatorname{Ir}^{191}$. $\quad Z$. Phys. 151(2):124-143, 1958. 


\section{Literaturverzeichnis}

[131] N. N. Greenwood und T. C. Gibb. Mössbauer spectroscopy Chapman and Hall Ltd, 1971.

[132] W. Friedrich, P. Knipping und M. Laue. Interferenzerscheinungen bei Röntgenstrahlen. Ann. Phys. 346(10):971-988, 1913.

[133] W. H. Bragg und W. L. Bragg. The reflection of x-rays by crystals. Proc. R. Soc. London, Ser. A 88(605):428-438, 1913.

[134] B. E. Warren. X-ray Diffraction Dover Pub., 1990.

[135] M. Knoll und E. Ruska. Das Elektronenmikroskop. Z. Phys. 78(5-6):318-339, 1932.

[136] D. B. Williams und C. B. Carter. Transmission Electron Microscopy Springer US, 1996.

[137] L. Hemmingsen, K. N. Sas und E. Danielsen. Biological applications of perturbed angular correlations of $\gamma$-ray spectroscopy. Chem. Rev. 104(9):4027-4062, 2004.

[138] T. Butz, G. M. Kalvius, H. Göbel und W. B. Holzapfel. The effect of high pressure on the electric field gradients at two inequivalent lattice sites of tantalum in $\omega$-zirconium. Hyperfine Interact. 1(1):1-14, 1975.

[139] P. de la Presa, R. E. Alonso, A. P. Ayala, V. V. Krishnamurthy, K. P. Lieb, A. López García, M. Neubauer und M. Uhrmacher. Phase transitions in the $\mathrm{SrHfO}_{3}$ perovskite measured with ${ }^{111} \mathrm{In} /{ }^{111} \mathrm{Cd}$ PAC. Hyperfine Interact. 120-121(1-8):479-483, 1999.

[140] V. V. Krishnamurthy, S. N. Mishra, M. R. Press und S. H. Devare. Local Magnetism of Dilute Ce Impurities in U Metal: Role of Ce- $4 f$, Host- $f$ Electron Interaction on $4 f$ Spin Fluctuations. Phys. Rev. Lett. 74(9):1661-1664, 1995.

[141] G. S. Collins, G. P. Stern und C. Hohenemser. Vacancy trapping in plastically deformed metals studied by hyperfine interactions. Phys. Lett. A 84(5):289-293, 1981.

[142] Th. Wichert. Hyperfine interactions - A tool for the study of lattice defects. Hyperfine Interact. 15(1):335-355, 1983.

[143] M. Deicher, G. Grübel, W. Reiner und Th. Wichert. Trapping of lattice defects at ${ }^{111} \mathrm{In}$ atoms in cold-worked copper. Mater. Sci. Forum 15-18:635-640, 1987.

[144] Th. Wenzel, M. Uhrmacher und K. P. Lieb. Point defects in NiO observed via perturbed angular correlation spectroscopy. J. Phys. Chem. Solids 55(8):683-697, 1994.

[145] M. Ferentz und N. Rosenzweig. Alpha-, beta- and gamma-ray spectroscopy, Seiten 1687-1690. North-Holland Publishing Company, 1965.

[146] L. C. Biedenharn und M. E. Rose. Theory of angular correlation of nuclear radiations. Rev. Mod. Phys. 25(3):729-777, 1953. 
[147] M. Nagl, U. Vetter, M. Uhrmacher und H. Hofsäss. A new all-digital time differential gamma-gamma angular correlation spectrometer. Rev. Sci. Instrum. 81(7):073501, 2010.

[148] M. Nagl. Next-Generation Perturbed Angular Correlation Spectroscopy Doktorarbeit, Universität Göttingen, 2014.

[149] M. A. Nagl, M. B. Barbosa, U. Vetter, J. G. Correia und H. C. Hofsäss. A new tool for the search of nuclides with properties suitable for nuclear solid state physics based on the Evaluated Nuclear Structure Data Files. Nucl. Instrum. Methods Phys. Res., Sect. A 726:17-30, 2013.

[150] M. Deicher, G. Grübel, E. Recknagel, Th. Wichert und D. Forkel. Detection of electronic perturbations in silicon after EC decay of ${ }^{111}$ In observed by PAC. Nucl. Instrum. Methods Phys. Res., Sect. B 13(1):499-502, 1986.

[151] S. Habenicht, D. Lupascu, M. Uhrmacher, L. Ziegeler und K.-P. Lieb. PAC-studies of Sn-doped $\operatorname{In}_{2} \mathrm{O}_{3}$ : electronic defect relaxation following the ${ }^{111} \mathrm{In}(\mathrm{EC}){ }^{111} \mathrm{Cd}$-decay. $Z$. Phys. B Con. Mat. 101(2):187-196, 1996.

[152] R. Firestone. Table of Isotopes, 8. Edition, 1996.

[153] J. Kajfosz. Calculations of EFG and PAC attenuation from randomly disturbed solutes in cubic lattices. Inst. of Nucl. Phys. Cracow Report No 858/PM:1-36, 1973.

[154] T. Butz. Analytic perturbation functions for static interactions in perturbed angular correlations of $\gamma$-rays. Hyperfine Interact. 52(3):189-228, 1989.

[155] D. Wegner. Calculated perturbed angular correlations for ${ }^{111}$ In doped cubic single crystals. Hyperfine Interact. 23(2):179-210, 1985.

[156] OEC GmbH. Szintillator-Kristalle NaI(Tl) / CsI(Tl) / CsI(Na) 24. April 2015. http://oec-gmbh.de/files/pdfs/detectors/szintillatorkristalle.pdf.

[157] W. Bolse. Untersuchungen der Stabilisierung von Strahlenschäden in Ag und AgIn durch Sauerstoff mittels der Methode der gestörten Gammawinkelkorrelation, 1982.

[158] Th. Wenzel. PAC-Untersuchungen an ${ }^{111} \mathrm{Cd}$ im System Titan-Sauerstoff, 1990.

[159] A. R. Arends, C. Hohenemser, F. Pleiter, H. de Waard, L. Chow und R. M. Suter. Data reduction methodology for perturbed angular correlation experiments. Hyperfine Interact. 8(1-3):191-213, 1980.

[160] M. Neubauer. Dynamische Hyperfeinwechselwirkung von ${ }^{111} \operatorname{In}(\mathrm{EC})^{111} \mathrm{Cd}_{\text {in }} \mathrm{Cr}_{2} \mathrm{O}_{3}$, 1993.

[161] T. Butz. Fouriertransformation für Fußgänger Vieweg+Teubner Verlag, 2011.

[162] E. Matthias, W. Schneider und R. M. Steffen. Some remarks on static electric quadrupole perturbations in angular correlations. Physics Letters 4(1):41-43, 1963. 


\section{Literaturverzeichnis}

[163] M. O. Zacate und H. Jaeger. Perturbed angular correlation spectroscopy-a tool for the study of defects and diffusion at the atomic scale. In Defect and Diffusion Forum Band 311, Seiten 3-38. Trans. Tech. Publ., 2011.

[164] M. Forker. The problematic of the derivation of the electric fieldgradient asymmetry parameter from TDPAC measurements or Mössbauer spectroscopy in imperfect crystal lattices. Nucl. Instrum. Methods 106(1):121-126, 1973.

[165] T. Butz, M. Ceolín, P. Ganal, P. Schmidt, M. A. Taylor und W. Tröger. A new approach in nuclear quadrupole interaction data analysis: cross-correlation. Phys. Scr. 54(3):234, 1996.

[166] G. Czjzek. Distribution of nuclear quadrupole splittings in amorphous materials and the topology of the $\left(\mathrm{V}_{z z}, \eta\right)$-parameter space. Hyperfine Interact. 14(3):189-194, 1983.

[167] T. Butz. Powder perturbation functions in angular correlations for distributions of electric field gradient tensor components: analytical expressions for $I=1$. Phys. Scr. 78(1):015801, 2008.

[168] M. A. Alves, N. Mommer, J. A. Gardner und W. E. Evenson. Simulated quadrupolar broadening in condensed matter PAC spectroscopy. Hyperfine Interact. 136-137(38):573-577, 2001.

[169] W. E. Evenson, M. Adams, A. Bunker, J. Hodges, P. Matheson, T. Park, M. Stufflebeam und M. O. Zacate. Inhomogeneous broadening of PAC spectra with $V_{z z}$ and $\eta$ joint probability distribution functions. Hyperfine Interact. 222(1-3):77-86, 2013.

[170] F. Heinrich. Untersuchung der Korrosion von HILUMIN-Batterieelektroden mittels zeitdifferentieller $\gamma$ - $\gamma$-Winkelkorrelationsspektroskopie, 2001.

[171] M. Uhrmacher, K. Pampus, F. J. Bergmeister, D. Purschke und K. P. Lieb. Energy calibration of the $500 \mathrm{kV}$ heavy ion implanter Ionas. Nucl. Instrum. Methods Phys. Res., Sect. B 9(2):234-242, 1985.

[172] M. Uhrmacher, M. Neubauer, W. Bolse, L. Ziegeler und K. P. Lieb. Preparation of ionimplanted and sub-monolayer ${ }^{111}$ In-tracer layers for perturbed angular correlation analysis. Nucl. Instrum. Methods Phys. Res., Sect. B 139(1-4):306-312, 1998.

[173] M. Kumakhov und F. Komarov. Energy loss and Ion ranges in solids. Gordon and Breach, Science Publishers, 1981.

[174] J. F. Ziegler, M. D. Ziegler und J. P. Biersack. SRIM-the stopping and range of ions in matter (2010). Nucl. Instrum. Methods Phys. Res., Sect. B 268(11):1818-1823, 2010.

[175] M. F. Perutz, M. G. Rossmann, A. F. Cullis, H. Muirhead und G. Will. Structure of hæmoglobin: a three-dimensional Fourier synthesis at 5.5-A. resolution, obtained by X-ray analysis. Nature 185:416-422, 1960. 
[176] G. K. Williamson und W. H. Hall. X-ray line broadening from filed aluminium and wolfram. Acta Metall. 1(1):22-31, 1953.

[177] B. E. Warren und B. L. Averbach. The effect of cold-work distortion on x-ray patterns. J. Appl. Phys. 21(6):595-599, 1950.

[178] W. Massa. Kristallstrukturbestimmung Teubner Verlag, 1996.

[179] Juan Rodríguez-Carvajal. Recent advances in magnetic structure determination by neutron powder diffraction. Physica B 192(1):55-69, 1993.

[180] H. M. Rietveld. Line profiles of neutron powder-diffraction peaks for structure refinement. Acta Crystallogr. 22(1):151-152, 1967.

[181] D. R. Black, D. Windover, A. Henins, J. Filliben und J. P. Cline. Certification of standard reference material 660B. Powder Diffr. 26(2):155-158, 2011.

[182] M. W. Barsoum, J. Golczewski, H. J. Seifert und F. Aldinger. Fabrication and electrical and thermal properties of $\mathrm{Ti}_{2} \mathrm{InC}, \mathrm{Hf}_{2} \mathrm{InC}$ and (Ti,Hf) $)_{2}$ InC. J. Alloys Compd. 340(12):173-179, 2002.

[183] S. Gupta und M. W. Barsoum. Synthesis and oxidation of $\mathrm{V}_{2} \mathrm{AlC}$ and $\left(\mathrm{Ti}_{0.5}, \mathrm{~V}_{0.5}\right)_{2} \mathrm{AlC}$ in air. J. Electrochem. Soc. 151(2):D24-D29, 2004.

[184] T. Scabarozi, A. Ganguly, J. D. Hettinger, S. E. Lofland, S. Amini, P. Finkel, T. ElRaghy und M. W. Barsoum. Electronic and thermal properties of $\mathrm{Ti}_{3} \mathrm{Al}\left(\mathrm{C}_{0.5}, \mathrm{~N}_{0.5}\right)_{2}$, $\mathrm{Ti}_{2} \mathrm{Al}\left(\mathrm{C}_{0.5}, \mathrm{~N}_{0.5}\right)$ and $\mathrm{Ti}_{2} \mathrm{AlN}$. J. Appl. Phys. 104(7):073713-073713, 2008.

[185] C. Brüsewitz, U. Vetter und H. Hofsäss. Determination of gradient elastic tensors: stress and strain dependencies of electric field gradients in cubic and hexagonal systems. J. Phys. Condens. Matter 27(5):055401, 2015.

[186] R.S. Raghavan und P. Raghavan. Anomalous temperature dependence of the quadrupole interaction in cadmium. Phys. Lett. A 36(4):313-314, 1971.

[187] E. N. Kaufmann, P. Raghavan, R. S. Raghavan, K. Krien und R. A. Naumann. Temperature dependence of the impurity electric quadrupole interaction: Cadmium and tantalum in titanium. Phys. Status Solidi B 63(2):719-726, 1974.

[188] P. Raghavan, R. S. Raghavan, E. N. Kaufmann, K. Krien und R. A. Naumann. Nuclear quadrupole interaction of ${ }^{111} \mathrm{Cd}$ in zinc (by PAC). J. Phys. F: Met. Phys. 4(4):L80, 1974.

[189] H. Wolf, S. Deubler, D. Forkel-Wirth, H. Foettinger, M. Iwatschenko-Borho, F. Meyer, M. Renn, W. Witthuhn und R. Helbig. Acceptors and donors in the wide-gap semiconductors $\mathrm{ZnO}$ and $\mathrm{SnO}_{2}$. Mater. Sci. Forum 10:863, 1986.

[190] L. Fast, J. M. Wills, B. Johansson und O. Eriksson. Elastic constants of hexagonal transition metals: Theory. Phys. Rev. B 51(24):17431-17438, 1995. 


\section{Literaturverzeichnis}

[191] M. Uhrmacher. Can PAC measurements be used to investigate defects in nanostructures? In Defect and Diffusion Forum Band 311, Seiten 105-133. Trans Tech Publ, 2011.

[192] A. Reuss. Berechnung der Fließgrenze von Mischkristallen auf Grund der Plastizitätsbedingung für Einkristalle. Z. Angew. Math. Mech. 9(1):49-58, 1929.

[193] K.-H. Hellwege und A. M. Hellwege. Landolt-Börnstein - Group III Condensed Matter: Numerical Data and Functional Relationships in Science and Technology. Springer Berlin Heidelberg, 1971.

[194] C. Brüsewitz, U. Vetter, H. Hofsäss und M.W. Barsoum. Perturbed angular correlation studies of uniaxial compressive stressed zinc, titanium, rutile, $\mathrm{Ti}_{2} \mathrm{AlN}$, and $\mathrm{Nb}_{2} \mathrm{AlC}$. $J$. Phys. Condens. Matter 26(29):295501, 2014.

[195] W. Witthuhn und W. Engel. Electric quadrupole interaction in noncubic metals. In Hyperfine Interactions of Radioactive Nuclei Topics in Current Physics, Kapitel 5, Seiten 205-289. Springer, 1983.

[196] S. J. Asadabadi, S. Cottenier, H. Akbarzadeh, R. Saki und M. Rots. Valency of rare earths in $R \mathrm{In}_{3}$ and $R \mathrm{Sn}_{3}$ : Ab initio analysis of electric-field gradients. Phys. Rev. B 66(19):195103, 2002.

[197] R.K. Sundfors, D.I. Bolef und P.A. Fedders. Nuclear acoustic resonance in metals and alloys: A review. Hyperfine Interact. 14(4):271-313, 1983.

[198] P. Raghavan. Table of nuclear moments. At. Data Nucl. Data Tables 42(2):189-291, 1989.

[199] B. Cheal, E. Mané, J. Billowes, M. L. Bissell, K. Blaum, B. A. Brown, F. C. Charlwood, K. T. Flanagan, D. H. Forest, C. Geppert, M. Honma, A. Jokinen, M. Kowalska, A. Krieger, J. Krämer, I. D. Moore, R. Neugart, G. Neyens, W. Nörtershäuser, M. Schug, H. H. Stroke, P. Vingerhoets, D. T. Yordanov und M. Žáková. Nuclear spins and moments of ga isotopes reveal sudden structural changes between $n=40$ and $n=50$. Phys. Rev. Lett. 104(25):252502, 2010.

[200] M. Radovic, A. Ganguly und M. W. Barsoum. Elastic properties and phonon conductivities of $\mathrm{Ti}_{3} \mathrm{Al}\left(\mathrm{C}_{0.5}, \mathrm{~N}_{0.5}\right)_{2}$ and $\operatorname{Ti} 2 \mathrm{Al}\left(\mathrm{C}_{0.5}, \mathrm{~N}_{0.5}\right)$ solid solutions. J. Mater. Res. 23(6):1517-1521, 2008.

[201] W. Voigt. Ueber die Beziehung zwischen den beiden Elasticitätsconstanten isotroper Körper. Ann. Phys. 274(12):573-587, 1889.

[202] P. A. Turner und C. N. Tomé. A study of residual stresses in zircaloy-2 with rod texture. Acta Metall. Mater. 42(12):4143-4153, 1994.

[203] Z. Budrovic, H. Van Swygenhoven, P. M. Derlet, S. Van Petegem und B. Schmitt. Plastic deformation with reversible peak broadening in nanocrystalline nickel. Science 304(5668):273-276, 2004. 
[204] A. S. Ingason, A. Mockute, M. Dahlqvist, F. Magnus, S. Olafsson, U. B. Arnalds, B. Alling, I. A. Abrikosov, B. Hjörvarsson, P. O. Å. Persson und J. Rosen. Magnetic selforganized atomic laminate from first principles and thin film synthesis. Phys. Rev. Lett. 110(19):195502, 2013.

[205] A. Mockute, M. Dahlqvist, J. Emmerlich, L. Hultman, J. M. Schneider, P. O. A. Persson und J. Rosen. Synthesis and ab initio calculations of nanolaminated $(\mathrm{Cr}, \mathrm{Mn})_{2} \mathrm{AlC}$ compounds. Phys. Rev. B 87(9):094113, 2013.

[206] S. Lin, P. Tong, B. S. Wang, Y. N. Huang, W. J. Lu, D. F. Shao, B. C. Zhao, W. H. Song und Y. P. Sun. Magnetic and electrical/thermal transport properties of Mn-doped $\mathrm{M}_{n+1} \mathrm{AX}_{n}$ phase compounds $\mathrm{Cr}_{2-x} \mathrm{Mn}_{x} \mathrm{GaC}(0<x<1)$. J. Appl. Phys. 113(5):053502, 2013.

[207] Q. Z. Tao, C. F. Hu, S. Lin, H. B. Zhang, F. Z. Li, D. Qu, M. L. Wu, Y. P. Sun, Y. Sakka und M. W. Barsoum. Coexistence of ferromagnetic and a re-entrant cluster glass state in the layered quaternary $\left(\mathrm{Cr}_{1-x}, \mathrm{Mn}_{x}\right)_{2} \mathrm{GeC}$. Mat. Res. Lett. 2(4):192-198, 2014.

[208] Z. Liu, T. Waki, Y. Tabata und H. Nakamura. Mn-doping-induced itinerant-electron ferromagnetism in $\mathrm{Cr}_{2}$ GeC. Phys. Rev. B 89(5):054435, 2014.

[209] A. Mockute, J. Lu, E. J. Moon, M. Yan, B. Anasori, S. J. May, M. W. Barsoum und J. Rosen. Solid solubility and magnetism upon $\mathrm{Mn}$ incorporation in the bulk ternary carbides $\mathrm{Cr}_{2} \mathrm{AlC}$ and $\mathrm{Cr}_{2}$ GaC. Mat. Res. Lett. 3(1):16-22, 2015.

[210] R. Salikhov, A. S. Semisalova, A. Petruhins, A. S. Ingason, J. Rosen, U. Wiedwald und M. Farle. Magnetic Anisotropy in the $\left(\mathrm{Cr}_{0.5} \mathrm{Mn}_{0.5}\right)_{2} \mathrm{GaC}$ MAX Phase. Mat. Res. Lett. Seiten 1-5, 2015.

[211] J. D. Makinson, J. S. Lee, S. H. Magner, R. J. De Angelis, W. N. Weins und A. S. Hieronymus. X-ray diffraction signatures of defects in nanocrystalline materials. Adv. X-Ray Anal. 42(C):407-411, 2000.

[212] T. Ungár und A. Borbély. The effect of dislocation contrast on x-ray line broadening: A new approach to line profile analysis. Appl. Phys. Lett. 69(21):3173-3175, 1996.

[213] I. C. Dragomir und T. Ungár. Contrast factors of dislocations in the hexagonal crystal system. J. Appl. Crystallogr. 35(5):556-564, 2002.

[214] M. Danek, K. F. Jensen, C. B. Murray und M. G. Bawendi. Synthesis of luminescent thin-film CdSe/ZnSe quantum dot composites using CdSe quantum dots passivated with an overlayer of ZnSe. Chem. Mater. 8(1):173-180, 1996.

[215] B. O. Dabbousi, J. Rodriguez-Viejo, F. V. Mikulec, J. R. Heine, H. Mattoussi, R. Ober, K. F. Jensen und M. G. Bawendi. (CdSe)ZnS core-shell quantum dots: synthesis and characterization of a size series of highly luminescent nanocrystallites. J. Phys. Chem. B 101(46):9463-9475, 1997. 


\section{Literaturverzeichnis}

[216] A. Mews, A. V. Kadavanich, U. Banin und A. P. Alivisatos. Structural and spectroscopic investigations of $\mathrm{CdS} / \mathrm{HgS} / \mathrm{CdS}$ quantum-dot quantum wells. Phys. Rev. B 53(20):R13242, 1996.

[217] S.-W. Kim, S. Fujita und S. Fujita. Self-organized $\mathrm{ZnO}$ quantum dots on $\mathrm{SiO}_{2} / \mathrm{Si}$ substrates by metalorganic chemical vapor deposition. Appl. Phys. Lett. 81(26):50365038, 2002.

[218] E. Sarigiannidou, E. Monroy, B. Daudin, J. L. Rouvière und A. D. Andreev. Strain distribution in GaN/AlN quantum-dot superlattices. Appl. Phys. Lett. 87(20):203112, 2005.

[219] B. Jogai. Three-dimensional strain field calculations in multiple InN/AlN wurtzite quantum dots. J. Appl. Phys. 90(2):699-704, 2001.

[220] G. N. Rao. Table of hyperfine fields for impurities in Fe, Co, Ni, Gd and Cr. Hyperfine Interact. 26(1-4):1119-1193, 1985. 


\section{Abbildungsverzeichnis}

1.1 Übersicht der Elemente, die MAX-Phasen bilden. . . . . . . . . . . . . . . . 2

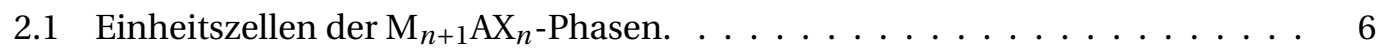

2.2 Elektronische Zustandsdichte in $\mathrm{Ti}_{2} \mathrm{AlN}$. . . . . . . . . . . . . 7

2.3 Typisches Verformungsverhalten in MAX-Phasen. . . . . . . . . . . . 9

2.4 Cadmium-p-Zustandsdichte in $\mathrm{Ti}_{2} \mathrm{AlN}$. . . . . . . . . . . . . . . 12

3.1 Zerfallsschema von ${ }^{111}$ In. . . . . . . . . . . . . . . . . . . . 26

3.2 Frequenzfaktoren und Amplituden als Funktion des Asymmetrieparameters. 27

3.3 Skizze des Messaufbaus. . . . . . . . . . . . . . . . . . . . . . . . 29

3.4 Beispiel eines PAC-Spektrums und des dazugehörigen Czjzek-Diagramms. . . 33

4.1 Struktur der Gradient-elastischen Tensoren $S_{m n}$ und $C_{m n} . \ldots \ldots 40$

4.2 Dehnungsinduzierte Feldgradienten in kubischen Metallen. . . . . . . . . . . 44

4.3 Dehnungsabhängigkeit des EFGs in hexagonalen Metallen. . . . . . . . . . . . 45

4.4 Dehnungsabhängigkeit des EFGs in MAX-Phasen. . . . . . . . . . . . . . 46

4.5 Dehnungsabhängigkeit des EFGs in Zinkoxid. . . . . . . . . . . . . . . 47

4.6 Dichtefunktion von $V_{z z}$ und $\eta$ in der Umgebung von Punktdefekten. . . . . . 51

4.7 Verbreiterung von $V_{z z}$ und $\eta$ durch Punktdefekte. . . . . . . . . . 51

4.8 Dichtefunktion von $V_{z z}$ und $\eta$ in der Umgebung von Stufenversetzungen. . . 54

4.9 Verbreiterung von $V_{z z}$ und $\eta$ durch Stufenversetzungen. . . . . . . . . . 54

4.10 Dichtefunktion von $V_{z z}$ und $\eta$ in der Umgebung von Schraubenversetzungen. 56

4.11 Dichtefunktion von $V_{z z}$ und $\eta$ in uniaxial belastetem polykristallinen $\mathrm{Ti}_{2} \mathrm{AlN}$. 60

4.12 Dichtefunktion von $V_{z z}$ und $\eta$ in uniaxial belastetem polykristallinen Titan. . 60

4.13 Elastische EFG-Verbreiterung in $\mathrm{Ti}_{2} \mathrm{AlN}$ und Ti. . . . . . . . . . . . . . . 61

4.14 Faltung der Dichtefunktion der elastischen Verbreiterung mit Gauß-Verteilung. 64

5.1 Einfluss uniaxialer Spannung auf das PAC-Spektrum. . . . . . . . . . . . 68

5.2 Verteilungsbreite $\delta$ nach verschiedenen Belastungszyklen in $\mathrm{Ti}_{2} \mathrm{AlN}$. . . . . 69

5.3 Änderung der Quadrupolkopplungskonstante unter uniaxialer Spannung. . . 71

5.4 Änderung der EFG-Verteilungsbreite mit zunehmender uniaxialer Spannung. 72

5.5 TEM-Aufnahme der $\mathrm{Cr}_{2}$ AlC-Dünnschichtprobe. . . . . . . . . . . . 73

5.6 PAC-Spektren von $\mathrm{Cr}_{2} \mathrm{AlC}$-Dünnschichtproben. . . . . . . . . . . . . . . 74

5.7 Temperaturabhängigkeit des EFGs in $\mathrm{Cr}_{2} \mathrm{AlC}$-Dünnschichtproben. . . . . . 75

5.8 Röntgendiffraktogramm von $\mathrm{Ti}_{2}\left(\mathrm{Al}_{0,5}, \mathrm{In}_{0,5}\right)$ C $\ldots \ldots \ldots \ldots 77$

5.9 PAC-Spektren im System $\left(\mathrm{Ti}_{1-x}, \mathrm{Nb}_{x}\right)_{2} \mathrm{AlC} \ldots \ldots \ldots \ldots$

5.10 PAC-Spektren im System $\left(\mathrm{Ti}_{1-x}, \mathrm{~V}_{x}\right)_{2}$ AlC $\ldots \ldots \ldots$. . . . . . . 80 


\section{Abbildungsverzeichnis}

5.11 PAC-Spektren im System $\operatorname{Ti}_{2}\left(\mathrm{Al}_{1-x}, \mathrm{In}_{x}\right) \mathrm{C} \ldots \ldots \ldots \ldots \ldots$. . . . . . . 81

5.12 PAC-Spektren im System $\operatorname{Ti}_{2} \operatorname{Al}\left(\mathrm{C}_{1-x}, \mathrm{~N}_{x}\right) \ldots \ldots \ldots$. . . . . . . . . . 82

5.13 Temperaturabhängigkeit des EFGs in den Mischkristallsystemen. . . . . . . . 83

5.14 Vergleich von EFG-Stärken und Gitterkonstanten in Mischkristallsystemen. . 84

5.15 Vergleich der EFG-Verteilungsbreite in Mischkristallsystemen. . . . . . . . . . 85

6.1 Asymmetrische Besetzung der p-Zustände in gedehntem Aluminium. . . . . 88

6.2 Korrelation zwischen berechneten und gemessenen Größen. . . . . . . . . . . 94

6.3 Abhängigkeit des elektrischen Feldgradienten vom $c / a$-Verhältnis. . . . . . . 96 


\section{Tabellenverzeichnis}

2.1 Elastische Konstanten der MAX-Phasen und anderer untersuchten Materialien. 8

3.1 Prozessparameter der MAX-Phasen. . . . . . . . . . . . . . . . . . 38

4.1 Berechnete Gradient-elastische Konstanten der kubischen Systeme. . . . . . . 48

4.2 Berechnete Gradient-elastische Konstanten der hexagonalen Systeme. . . . . . 48

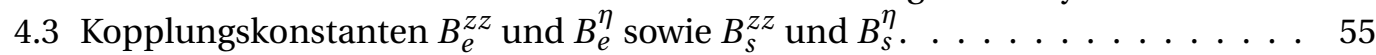

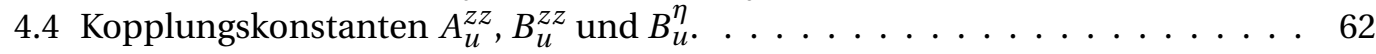

5.1 Übersicht der Gitterkonstanten untersuchter Materialien. . . . . . . . . . . . 66

5.2 Experimentelle Spannungsabhängigkeit des EFGs. . . . . . . . . . . . . . . 70

5.3 Vergleich zwischen EFG-Parametern in Volumen- und Dünnschichtproben. 76

5.4 EFG-Parameter der Mischkristallsysteme im Vergleich. . . . . . . . . . . . . 84

6.1 Vergleich berechneter und experimenteller Gradient-elastischer Konstanten. . 91

6.2 Berechnete und experimentelle Spannungsabhängigkeiten des EFGs. . . . . . 92

A.1 Details der DFT-Rechnungen kubischer Systeme. . . . . . . . . . . . . . . . 115

A.2 Details der DFT-Rechnungen hexagonaler Systeme. . . . . . . . . . . . . . 116 



\section{Publikationen}

- D. Jürgens, M. Uhrmacher, H. G. Gehrke, M. Nagl, U. Vetter, C. Brüsewitz, H. Hofsäss, J. Mestnik-Filho und M. W. Barsoum. Electric field gradients at ${ }^{111} \mathrm{In} /{ }^{111} \mathrm{Cd}$ probe atoms on A-sites in 211-MAX phases. J. Phys. Condens. Matter, 23(50):505501, 2011.

2013

- C. Brüsewitz, I. Knorr, H. Hofsäss, M. W. Barsoum und C. A. Volkert. Single crystal pillar microcompression tests of the MAX phases $\mathrm{Ti}_{2} \mathrm{InC}$ and $\mathrm{Ti}_{4} \mathrm{AlN}_{3}$. Scr. Mater, 69(4):303-306, 2013.

- Y. Xu, K. Zhang, C. Brüsewitz, X. Wu und H. Hofsäss. Investigation of the effect of low energy ion beam irradiation on mono-layer graphene. AIP Adv., 3(7):072120, 2013.

- H. Hofsäss, K. Zhang, H. G. Gehrke und C. Brüsewitz. Propagation of ripple patterns on Si during ion bombardment. Phys. Rev. B, 88(7):075426, 2013.

- C. Brüsewitz, U. Vetter, H. Hofsäss und M. W. Barsoum. Perturbed angular correlation studies of uniaxial compressive stressed zinc, titanium, rutile, $\mathrm{Ti}_{2} \mathrm{AlN}$, and $\mathrm{Nb}_{2} \mathrm{AlC}$. $J$. Phys. Condens. Matter, 26(29):295501, 2014.

- C. Brüsewitz, U. Vetter und H. Hofsäss. Determination of gradient elastic tensors: stress and strain dependencies of electric field gradients in cubic and hexagonal systems. J. Phys. Condens. Matter, 27(5):055401, 2015.

- M. K. Mahata, T. Koppe, T. Mondal, C. Brüsewitz, K. Kumar, R. V. Kumar, H. Hofsäss und U. Vetter. Incorporation of $\mathrm{Zn}^{2+}$ ions into $\mathrm{BaTiO}_{3}: \mathrm{Er}^{3+} / \mathrm{Yb}^{3+}$ nanophosphor: an effective way to enhance upconversion, defect luminescence and temperature sensing. Phys. Chem. Chem. Phys., 17(32):20741-20753, 2015.

- C. Beckmann, K. Zhang, H. Hofsäss, C. Brüsewitz, U. Vetter und K. Bharuth-Ram. Mössbauer Spectroscopy study of Surfactant Sputtering induced Fe Silicide formation on a Si surface. Appl. Surf. Sci., 357(A):493-497, 2015. 


\section{Lebenslauf}

Persönliches:

$\begin{array}{ll}\text { Name: } & \text { Christoph Brüsewitz } \\ \text { Geburtsdatum: } & \text { 28. Juli } 1986 \\ \text { Geburtsort: } & \text { Groß-Umstadt } \\ \text { Staatsangehörigkeit: } & \text { deutsch } \\ \text { Familienstand: } & \text { ledig }\end{array}$

\section{Bildungsweg:}

1992 - 1996: Markwaldschule Langstadt

1996 - 1998: Geiersbergschule Groß-Umstadt

1998 - 2005: Max-Planck-Gymnasium Groß-Umstadt, Abitur

2006 - 2009: Georg-August-Universität Göttingen, B.Sc. Physik

2009 - 2011: Georg-August-Universität Göttingen, M.Sc. Physik

2012 - 2015: Georg-August-Universität Göttingen, Dr. rer. nat. 


\section{Danksagung}

Ich möchte die Gelegenheit nutzen, all den Personen zu danken, die einen Beitrag zur Entstehung dieser Arbeit geleistet haben.

Mein besonderer Dank gilt meinem Betreuer Herrn Prof. Hans C. Hofsäss, der mir in den letzten Jahren all die wissenschaftlichen Freiheiten zugestanden hat, welche die breite Aufstellung dieser Arbeit ermöglichten.

Frau Prof. Cynthia A. Volkert möchte ich für den wissenschaftlichen Dialog und die Bereitschaft danken, als Korreferentin diese Arbeit zu begutachten. Gleichermaßen gilt mein Dank dem zweiten Korreferenten, Prof. Peter Blaha, und den weiteren Mitgliedern der Prüfungskommission.

Herrn Dr. Ulrich Vetter möchte ich für die zahlreichen Ratschläge und die fortwährende Unterstützung der letzten Jahre danken. Herrn Dr. Michael Uhrmacher danke ich für für seine nützlichen Hinweise zur PAC-Methode und seine Unterweisung im praktischen Umgang mit radioaktiven Stoffen. In diesem Zusammenhang möchte ich neben Herrn Dr. Uhrmacher auch Herrn Dr. Lüder Ziegeler für die Unterstützung bei den Vorbereitungen zur Indiumimplantation sowie Herrn Detlef Purschke für den Betrieb des Ionenbeschleunigers danken.

I am thankful to Prof. Michel W. Barsoum for his generous hospitality at Drexel University and his willingness for providing most of the bulk samples used herein. I would also like to thank Prof. Vladimir Vishnyakov for providing the thin film samples and Prof. Stefaan Cottenier for helpful discussions on the electronic structure of transition metals. I am indebted to Darin J. Tallman, Mohamed Shamma, Michael Naguib, Elad N. Caspi, and Zhorro Nikolov from Drexel University for help with the sample preparation and XRD measurements.

Meinen Freunden und Kollegen der Arbeitsgruppe, Julian A. Amani, Tristan Koppe, Dr. Matthias A. M. Nagl, Alexander Rahn und Dr. Kun Zhang möchte ich für die kontinuierliche und vielfältige Hilfestellung zu unterschiedlichsten Aspekten dieser Arbeit danken. Gleichermaßen gilt mein Dank den ehemaligen Mitgliedern des Instituts, Dr. Marc Brötzmann, Dr. Hans-Gregor Gehrke, Dr. Daniel Jürgens und Martina Schulte-Borchers, sowie Dr. Inga Knorr, die mich in eine Vielzahl experimenteller Methoden einführten.

Der feinmechanischen Werkstatt des II. Physikalischen Instituts, der Elektronikwerkstatt und der Zentralwerkstatt möchte ich für die technische Unterstützung dieser Arbeit danken. Dem Sekretariat des Instituts gilt mein Dank für die Unterstützung bei der Bewältigung des bürokratischen Alltags. Für die Bereitstellung und Wartung der Großrechner der GWDG möchte ich Dr. Christian Boehme und Tim Ehlers herzlichst danken.

Herrn Prof. Ottmar Kanert bin ich für die Bereitschaft dankbar, mir eine Übersicht Gradient-elastischer Konstanten kubischer Systeme zur Verfügung zu stellen.

Diese Arbeit wurde von der Deutschen Forschungsgemeinschaft unter der Nummer HO 1125/19-2 gefördert. 